Portland State University

PDXScholar

8-2-2021

\title{
Opportunities and Challenges in the Collection and Use of Socio-Spatial Data in National Forest Planning
}

Diane Trechsel Besser

Portland State University

Follow this and additional works at: https://pdxscholar.library.pdx.edu/open_access_etds

Part of the Forest Management Commons

Let us know how access to this document benefits you.

Recommended Citation

Besser, Diane Trechsel, "Opportunities and Challenges in the Collection and Use of Socio-Spatial Data in National Forest Planning" (2021). Dissertations and Theses. Paper 5763.

https://doi.org/10.15760/etd.7634

This Dissertation is brought to you for free and open access. It has been accepted for inclusion in Dissertations and Theses by an authorized administrator of PDXScholar. Please contact us if we can make this document more accessible: pdxscholar@pdx.edu. 
Opportunities and Challenges in the Collection and Use of Socio-Spatial Data in National Forest Planning by

Diane Trechsel Besser

A dissertation submitted in partial fulfilment of the requirements for the degree of

\author{
Doctor of Philosophy \\ in \\ Public Affairs and Policy \\ Dissertation Committee: \\ Craig Shinn, Chair \\ Jennifer Allen \\ Rebecca McLain \\ Max Nielsen-Pincus \\ Vivek Shandas
}

Portland State University

2021 


\begin{abstract}
Understanding human-environment connections to places is an important component of land-use management. Tools for collecting geographically referenced public values-based data (defined as socio-spatial data) for use in natural resource planning have been reported in academic journals for decades. The utility of sociospatial data is in making public values tangible and potentially actionable in land-use analyses and decision processes. However, there is a lack of comprehensive documentation on the ways in which socio-spatial data is perceived, collected, interpreted and applied at a practical level. A better understanding of these factors allows planners to mitigate barriers and leverage opportunities to more effectively collect and incorporate public values into planning.

Using the U.S. Forest Service (USFS) as a case study, this research explores the barriers and opportunities in incorporating socio-spatial data into land-use decisions, focusing on the forest plan revision process. Applied thematic analysis is used to identify themes derived from interviews with USFS personnel at research stations, regional offices and a sample of national forests. Findings indicate forest planners collect and apply this type of data using a diverse suite of tools, at numerous points in the process, and this data impacts decisions in direct and indirect ways. Socio-spatial data were used to identify special places, mediate conflicting use preferences, assess and revise proposed boundary areas, and inform standard analyses, such as the recreation opportunity spectrum.
\end{abstract}

Budget issues that directly impact staff capacity are the most pressing barriers, creating a scarcity of social scientists within the agency that reverberates through the 
system and hinders the ability to collect and use socio-spatial data. However, opportunities exist in leveraging existing participatory processes to expand collection of socio-spatial data beyond the forest plan revision process, such as using the USFS's Talking Points Collaborative Mapping application. More expansive use of the tool will make visible the utility of socio-spatial data. Recommendations include additional research, such as using contingency theory to delve deeper into the impacts of decisions, particularly focusing on the impacts of trade-offs on the integration of public values into planning documents. Educators also play a key role in advocating for professional development courses that focus on public values in natural resource planning and highlight the utility of socio-spatial data in this context. This would not only infuse skills needed in the workforce, but also establish use of socio-spatial data in decision-making as a best practice in natural resource management. 


\section{ACKNOWLEDGEMENTS}

Completing a dissertation is a journey of scholarly and personal exploration in the truest sense. It is never a journey taken alone. I owe many thanks to many people. In particular, I would like to acknowledge Dr. Craig Wollner, alav ha-shalom. His untimely passing means that he will not see the culmination of my work, but he believed in me and convinced me to take those first adventurous, and admittedly scary, steps toward a Ph.D. in public affairs and policy so many years ago. I know he is smiling and wishing he could tell me "I told you so!" To Dr. Meg Merrick, a cherished colleague, who shares with me a love of geography as well as a deep understanding of the empowering potential of maps and socio-spatial data for public agencies and communities. To Dr. Lee Cerveny, at the USFS Pacific Northwest Research Station. Her unwavering resolve to better understand human-environment connections in natural resource planning gave purpose and direction to my professional and academic journey at all stages. To my committee members: Dr. Craig Shinn, chair, who helped me navigate all the twists and turns of completing a dissertation, not to mention encouraging and nudging me in the right direction when I became lost in the weeds or overwhelmed by the expansiveness of my task and Drs. Jennifer Allen, Max Nielsen-Pincus and Vivek Shandas. I so appreciate their knowledge, insights and scholarly perspectives that helped me cross the finish line. And, finally, special thanks to Dr. Rebecca McLain, committee member and mentor. She unlocked the gate that launched me on this journey and has walked with me ever since.

And, of course, I would not have gotten to this point without the unwavering love, support and encouragement of my son, Aaron, who provided the sweetest words one can hear - way to go, Mom! 


\section{TABLE OF CONTENTS}

ABSTRACT

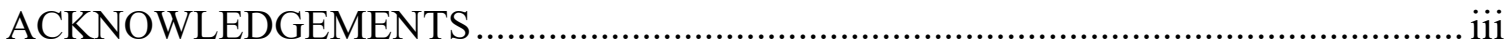

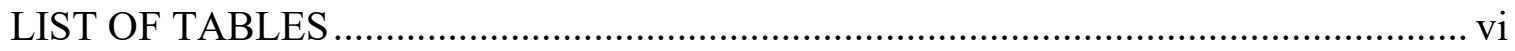

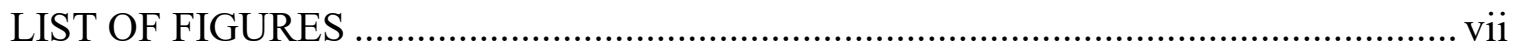

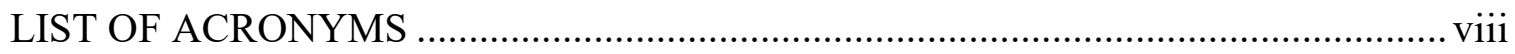

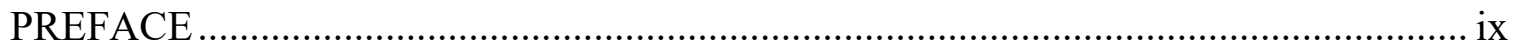

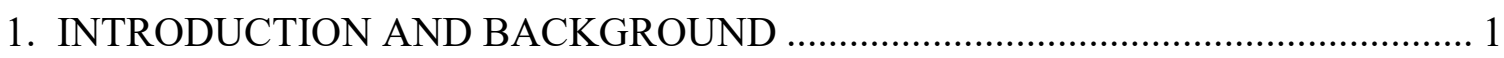

1.1 Human Dimensions of Land-Use Planning...................................................... 3

1.2 The Evolution of Forest Planning, Sense of Place and Geospatial Technology ... 5

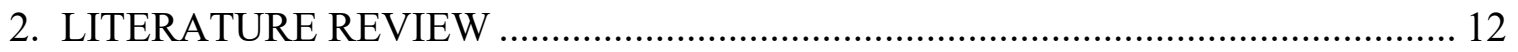

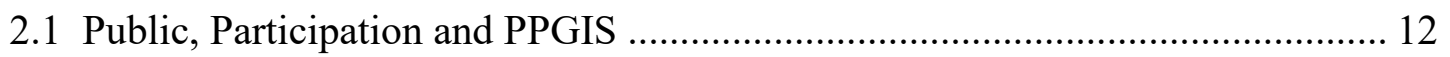

2.2 PPGIS Strategies and Case Studies................................................................. 14

2.3 Problem Statement and Research Question .................................................... 16

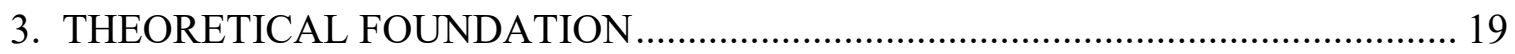

3.1 General Systems Theory and Systems Science................................................ 19

3.2 Structural Contingency Theory …………………….................................... 23

3.3 Initial Conceptual Framework....................................................................... 32

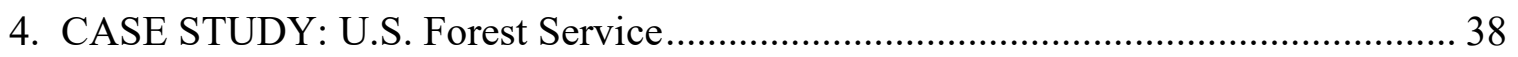

4.1 U.S. Forest Service Organizational Structure …………………………........... 40

4.2 Comprehensive Forest Management Plan Revision Process ............................... 43

4.3 U.S. Forest Service Research Stations .................................................................. 49

4.4 Paperwork Reduction Act and Office of Management and Budget Oversight ... 50

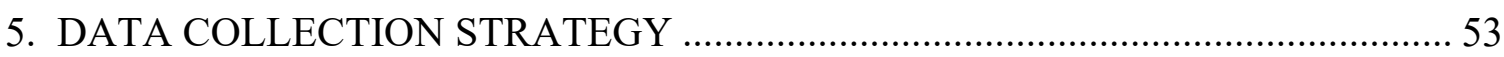

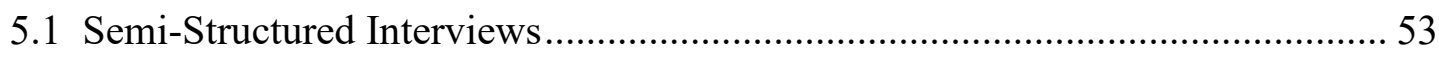

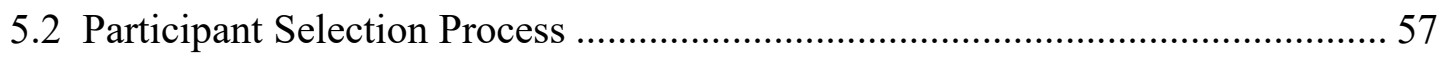

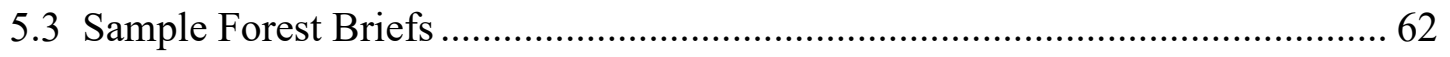

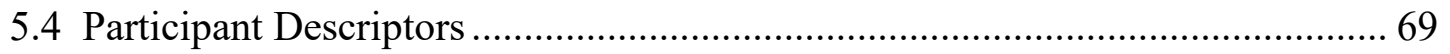

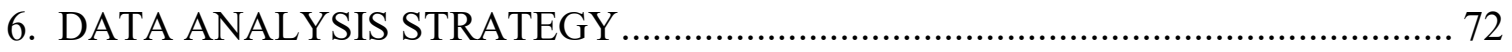

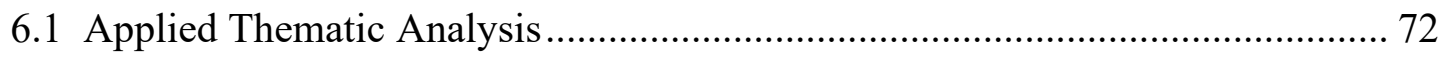

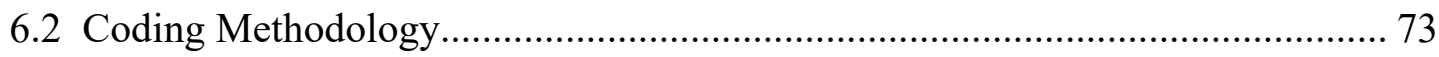

6.3 Developing Themes..................................................................................... 83 


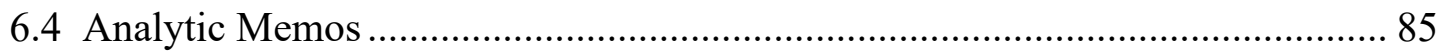

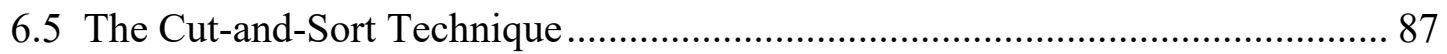

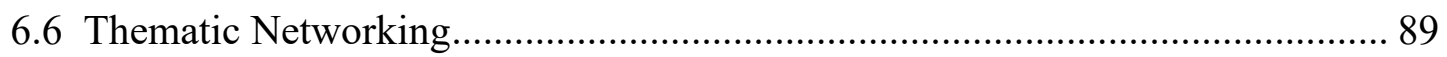

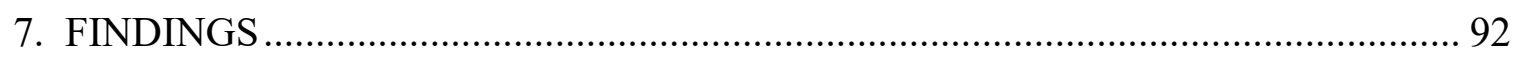

7.1 The Spectrum of Socio-Spatial Data ............................................................. 92

7.2 Salient Themes in Coded Text Segments......................................................... 104

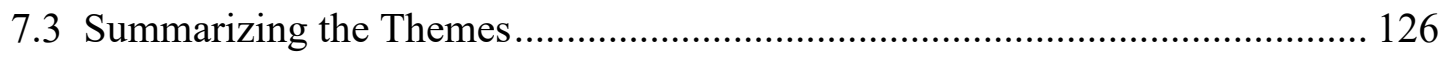

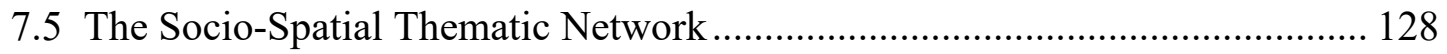

7.6 Identifying Barriers, Challenges and Opportunities.......................................... 131

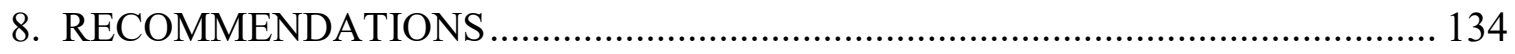

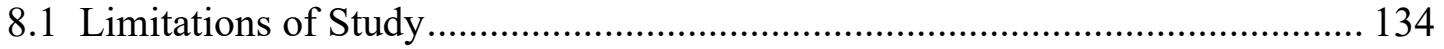

8.2 Recommendations for Practitioners …………………................................. 137

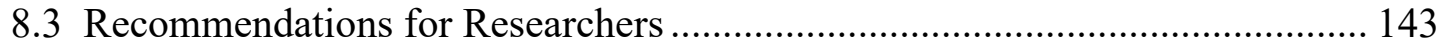

8.4 Recommendations for Policy ……….......................................................... 147

8.5 Recommendations for Educators and other Professionals ................................. 148

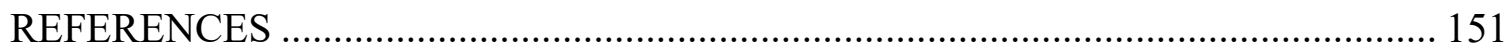

\section{APPENDICES}

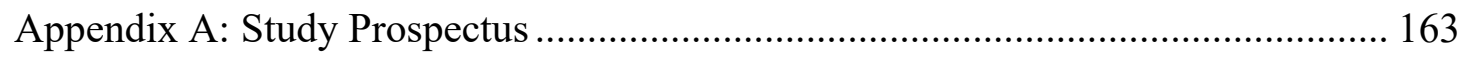

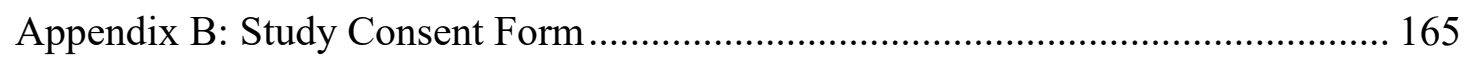

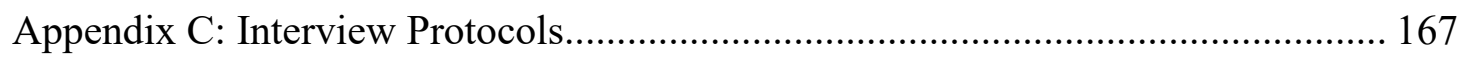

Appendix D: Flathead National Forest Brief ...................................................... 175

Appendix E: Colville National Forest Brief ......................................................... 180

Appendix F: Coconino National Forest Brief....................................................... 187

Appendix G: Francis Marion National Forest Brief ................................................ 193

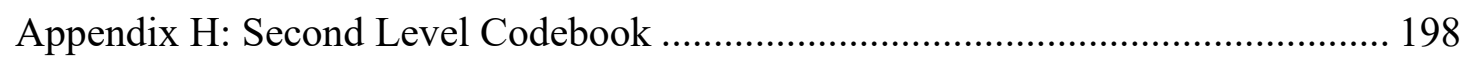

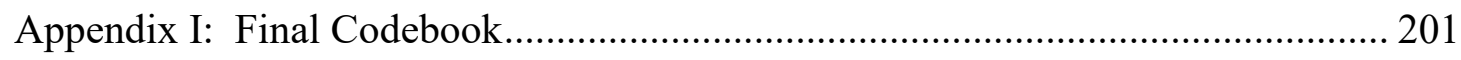

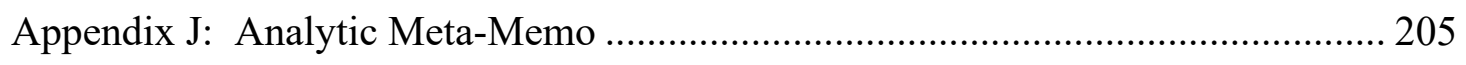

Appendix K: Thematic Network Organizing Criteria …………………………..... 213 


\section{LIST OF TABLES}

Table 1: Advantages and limitations of the inductive research approach.................. 33

Table 2: Advantages, limitations and mitigation strategies in semi-structured interviewing

Table 3: National forests conducting forest plan revisions (as of Nov. 2015) and associated rankings based on the study's selection criteria.

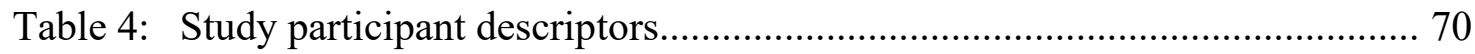

Table 5: First level parent codes with description and example text....................... 75

Table 6: Aggregate number of coded segments by first level code and organization level.

Table 7: Parent structural codes and second level sub-codes................................... 79

Table 8: Tools used to collect socio-spatial data during forest plan revision by sample forest.

Table 9: Summary of themes in the collection and use of socio-spatial data by thematic statement and keyword 


\section{LIST OF FIGURES}

Figure 1: The role of congruence in structural contingency theory............................. 26

Figure 2: A classification matrix of equifinality scenarios...................................... 30

Figure 3: Schematic of the study's initial conceptual framework............................ 35

Figure 4: U.S. Forest Service, National Forest System administrative levels............ 41

Figure 5: U.S. Forest Service forest management plan revision process.................. 44

Figure 6: The process of applied thematic analysis............................................. 72

Figure 7: Socio-spatial data collection tools in forest planning ............................. 94

Figure 8: The socio-spatial data in forest planning thematic network...................... 129 


\section{LIST OF ACRONYMS}

$\begin{array}{ll}\text { BAS/BASI } & \text { Best Available Science/Best Available Scientific Information } \\ \text { EIS } & \text { Environmental Impact Statement (DEIS=Draft; FEIS=Final) } \\ \text { FACA } & \text { Federal Advisory Committee Act } \\ \text { GIS } & \text { Geographic Information Systems } \\ \text { HEM } & \text { Human Ecology Mapping } \\ \text { ID Team } & \text { Interdisciplinary Forest Planning Team } \\ \text { INFRA } & \text { Infrastructure Model } \\ \text { IMPLAN } & \text { Impact Analysis for Planning (socio-economic modeling) } \\ \text { LVM } & \text { Landscape Values Mapping } \\ \text { MUSY } & \text { Multiple Use Sustained Yield Act of 1960 } \\ \text { NEPA } & \text { National Environmental Policy Act of 1969 } \\ \text { NFMA } & \text { National Forest Management Act of 1976 } \\ \text { NFS } & \text { National Forest System } \\ \text { NOI } & \text { Notice of Intent (to revise forest management plan) } \\ \text { NSRE } & \text { National Survey of Recreation \& the Environment } \\ \text { NVUM } & \text { National Visitor Use Monitoring Survey } \\ \text { OHV } & \text { Off-Highway Vehicle } \\ \text { OMB } & \text { Congressional Office of Management \& Budget } \\ \text { PPGIS } & \text { Public Participatory Geographic Information System } \\ \text { PRA } & \text { Paperwork Reduction Act of 1980 } \\ \text { ROD } & \text { Record of Decision (submission of decision in the Federal Register) } \\ \text { ROS } & \text { Recreation Opportunity Spectrum Model } \\ \text { SMS } & \text { Scenery Management System } \\ \text { TEAM } & \text { Enterprise TEAM (USFS professional contracting service) } \\ \text { TPCM } & \text { Talking Points Collaborative Mapping Tool } \\ \text { USDA } & \text { U.S. Department of Agriculture } \\ \text { USFS } & \text { U.S. Forest Service } \\ \text { VCA } & \text { Values Compatibility Analysis (GIS-based tool) } \\ \text { VGI } & \text { Volunteered Geographic Information (a.k.a. Crowdsourcing; Web2.0) } \\ \text { WOS } & \text { Wilderness Opportunity Spectrum Model }\end{array}$




\title{
PREFACE \\ Opportunities and Challenges in the Collection and Use of Socio-Spatial Data in National Forest Planning
}

\author{
Diane T. Besser \\ Portland State University, July 2021

\section{EXECUTIVE SUMMARY}

Understanding human-environment connections to places has become an important component of land-use decisions (Bauer, 2020; Rose et al., 2020; Szymkowiak, 2021). People attach values to special places or specific types of landscapes that influence opinions about the appropriateness of different types of land-use. This study explores the barriers and opportunities in incorporating data about public place-based values into land-use decisions within the context of national forest planning.

Problem Statement and Research Question. Tools and strategies for collecting geographically referenced public values-based data (defined here as socio-spatial data) for use in natural resource planning have been reported in academic journals for decades. However, there is a lack of comprehensive documentation on the ways in which sociospatial data is perceived, collected, interpreted and applied at a practical level in making land-use decisions. Using the U.S. Forest Service (USFS) as a case study, the goal of this research is to identify significant factors that represent barriers and opportunities in the collection and use of socio-spatial data within the context of the national forest management plan revision process. A better understanding of these factors allows planners to mitigate barriers and leverage opportunities to more effectively collect and incorporate public values into land-use decisions. 
Methods. This research uses an exploratory inductive approach. An initial conceptual framework identifies potential factors organized into four categories: (1) structural (formal organizational processes); (2) perceptual (knowledge about and attitudes toward socio-spatial data); (3) cultural (habits of practice); and (4) external (laws, public sentiments). General systems science is used to inform the study design, organize themes that emerge, and visualize interrelationships. Structural contingency theory provides a framework in which to interpret insights derived from the themes as internal and external factors agency decision-makers are responding to in order to complete their task (revising a forest plan).

Semi-structured interviews were conducted with USFS social scientists at research stations and regional offices as well as with forest-level staff representing the Flathead, Coconino, Colville and Francis Marion National Forests. The final dataset compiles participant responses from 23 total interviews - five participants at regional offices, five at research stations, and 13 forest-level staff. Applied thematic analysis methods were employed to extract meta-themes from the dataset. Specific strategies include iterative coding to organize the data based on the categories identified in the conceptual framework and thematizing tools (analytic memo, cut-and-sort and thematic networking) to identify salient themes and linkages between them.

Findings. This research began with an observation of a need to have formal and comprehensive documentation of how socio-spatial data is being integrated into forest planning processes and what barriers exist or opportunities that planners are leveraging. The first findings indicate that forest planners collect and apply this type of data using a 
diverse suite of tools, at numerous points in the process, and this data impacts decisions in direct and indirect ways. This is represented in the first set of themes as listed below:

1. Socio-spatial data collection tools take many forms and produce different kinds of socio-spatial data.

2. Socio-spatial data collection tools are employed at different points in forest planning and for different purposes.

3. Socio-spatial data influences decision-making in both direct and indirect ways.

Planners apply numerous static and online mapping tools to collect socio-spatial data, embedded in their public participation strategies. Socio-spatial data were used to identify special places, mediate conflicting use preferences, assess (and revise) proposed boundary areas, and to inform standard analyses, such as the recreation opportunity spectrum.

The remaining themes that emerged from participant responses are organized into three groups - those that represent significant barriers, those that identify challenges (difficult, but not insurmountable), and a third that suggests potential opportunities for more extensive collection and use of socio-spatial data.

\section{Themes Considered Barriers:}

4. The scarcity of staff with experience and skills in social science and/or PPGIS [public participatory geographic information systems] limits the capacity to collect socio-spatial data.

5. Budget reductions are directly related to lack of staff capacity.

6. Organizational leadership acts as an enabling factor; supportive leadership results in effective collection and integration of socio-spatial data.

Significant barriers, perceived as intractable by study participants, are associated with limited staff capacity resulting from budget reductions. Budget reductions lead to insufficient staffing, particularly in the social sciences, which results in lack of staff time to collect and/or use socio-spatial data. Residual connections occur around the role of 
leadership, as it relates to decisions regarding what planning strategies will be employed

or where resources will be allocated given limited funds and capacity.

\section{Themes Considered Challenges:}

7. There are significant socio-spatial data gaps around data at the local scale; sociospatial data retrieved from non-traditional sources may address this data gap.

8. A social science support portal is needed to effectively transmit knowledge about the collection and use of socio-spatial data in planning.

9. The culture of expertise and data-driven planning within the USFS hinders forest planning where issues should guide the process; this has the potential to deprioritize socio-spatial data that can shed light on these issues.

10. Interdisciplinary work is valued within the agency, but expertise siloing and language barriers hinder a better understanding of the utility of socio-spatial data in informing land-use decisions.

Themes representing challenges revolve around science and data dissemination.

They include data gaps (around localized data) and the need for a data portal for better sharing of information and best practices. These themes are directly impacted by staff capacity issues; participants noted they simply lacked the time to create and maintain a data portal or add extra socio-spatial data collection tasks to their already full plates. In addition, participants spoke about challenges around developing the language and culture for natural and social scientists to work better together and that a data-driven culture within the agency has the potential to deprioritize qualitative socio-spatial data and its utility in informing planning decisions and improving public engagement.

\section{Themes Considered Opportunities:}

11. The PRA [Paperwork Reduction Act] and OMB [Office of Management \& Budget] regulations represent a significant barrier to the collection of socio-spatial for research purposes, but not for on-the-ground planners who consider sociospatial data collection as public input.

12. Public engagement is a necessary and on-going activity in forest planning with numerous opportunities to collect data on public values and preferences beyond forest plan revision.

13. Socio-spatial data belongs in the public sensing phase of planning to better engage the public and their contribution to the Need for Change and Desired Conditions statements. 
A key insight is that forest planners consider socio-spatial data as publicly-derived data, rather than scientifically-derived data. Semantics, perhaps, but this opens up numerous opportunities to collect socio-spatial data during any public participatory activity taking place in the forests. Expanding the collection of socio-spatial data in diverse public contexts can reach different constituencies and address gaps in knowledge about local values and uses of forest resources that traditional, more coarse, data sources (e.g. U.S. Census) cannot provide.

Recommendations. Participants offered numerous suggestions on how to expand the socio-spatial data collection toolkit including more expansive use of participatory citizen-science, crowdsourcing and volunteered geographic information (VGI). In addition, more extensive use of the Talking Points Collaborative Mapping Tool or TPCM (an online mapping application approved by the USFS for public engagement) would contribute to embedding collection of socio-spatial data into standard planning practice.

For researchers, questions derived from the themes suggest that internal and external factors are present affecting decisions about how much and what types of sociospatial data can (or should) be collected during the planning process. Planners must navigate conflicting contingencies, such as societal and political pressure to be efficient and cost-effective, while also being flexible and accommodating in the face of contentious public attitudes around land-use preferences. Trade-offs are inevitable. Further research that isolate contingencies identified in these themes and assess impacts of decisions based on a performance objective (such as public acceptance of land-use decisions) would add to the body of knowledge around addressing human-environment 
connections or mitigating conflicting public values and use preferences in natural resource planning.

Educators can advocate for professional development courses that focus on public values in natural resource planning and highlight the utility of socio-spatial data in this context. This would not only infuse skills needed in the workforce to collect and interpret socio-spatial data, but also establish use of socio-spatial data in decision-making as a best practice in natural resource management.

Simple policy statements can also be effective in advancing the use of sociospatial data. Policies are operational in nature; they are statements of intent that translate into protocols. Any formal policy that directly addresses socio-spatial data adds a new factor decision-makers must consider as they allocate budgets, assign staff time and determine planning priorities. A simple internal policy statement, that says something like "the XXXXX National Forest has adopted the use of TPCM to collect public input on values and uses of our forest resources," is a policy of few words. However, built into this statement is a commitment to invest in building staff capacity to employ the tools and to use the socio-spatial data they produce.

Collecting and integrating socio-spatial data into natural resource planning allows land managers to better understand public values and use of landscapes and develop action plans that create a shared sense of stewardship of public lands. This sentiment is aptly summed up by Black and Liljeblad (2008).

...embedded in the concept of place is a story about ecological function and appropriateness of human actions. Only by articulating these stories can we begin to illuminate the fundamental basis of conflict over appropriate land management practices. By placing these stories in an objective, generalizable framework, we can provide managers and the interested public the means to 
develop a deeper understanding of their relationships to place and thus, the opportunity to co-create new stories that may be even more appropriate for guiding behavior (both as federal managers and private individuals) in their current and future landscapes. (Black \& Liljeblad, 2008, p. 1)

\section{References}

Black, A. E. \& Liljeblad, A. (2008). Integrating Social Values in Vegetation Models via GIS: The Missing Link for the Bitterroot National Forest. Final Report to the Joint Fire Science Program (JFSP Project No. 04-2-1-114).

Bauer, J. W. R., Ries, P., \& Rosenberger, R. S. (2020). A relationship between emotional connection to nature and attitudes about urban forest management. Urban Ecosystems 23(1): 187-197.

Rose, L., Hallemeier, J. \& Colburn, K. (2020). National forest planning: applying new technologies and approaches to improve public participation and decisionmaking. In: Selin, S., Cerveny, L. K., Blahna, D. J. \& Miller, A. B., eds. (2019). Igniting Research for Outdoor Recreation: Linking Science, Policy, and Action. Portland, OR: U.S. Department of Agriculture, Forest Service, Pacific Northwest Research Station (GTR-PNW-987).

Szymkowiak, M. (2021). A conceptual framework for incorporating human dimensions into integrated ecosystem assessments. Frontiers in Marine Science 8: 1-14. 


\section{INTRODUCTION AND BACKGROUND}

Understanding human-environment connections to places has become an important component of land-use decisions (Bauer, 2020; Biedenweg \& Nelson, 2017; Hall et al., 2009; Rose et al., 2020; Szymkowiak, 2021). People attach values to special

places or specific types of landscapes that influence opinions about the appropriateness of different types of land-use. This study explores the barriers and opportunities in incorporating data about public place-based values into land-use decisions within the context of national forest planning.

Geographically referenced (i.e. mapped) values- and use-based data collected from the public (defined here as socio-spatial data) for use in natural resource planning have been reported in academic journals for decades. While these published works have demonstrated the efficacy of integrating socio-spatial data into planning, authors have noted there is a lack of comprehensive documentation, or evidence, on the ways in which socio-spatial data is perceived, collected, interpreted and applied at a practical level in making land-use decisions (Bennett et al., 2016; Brown et al., 2020; Brown \& Donovan, 2014; Brown \& Fagerholm, 2015). To address this gap, the U.S. Forest Service (USFS) is used as a case study in order to provide this documentation and to identify significant factors that influence the collection and integration of socio-spatial data within the context of the national forest management plan revision process.

Chapter 1 provides background and context around the human dimension of landuse planning, focusing on the concept of sense of place, as well as a brief historical account of the integration of sense of place into forest planning and the use of data collection tools, such as public participatory geographical information systems (PPGIS). 
Chapter 2 contains a brief literature review focusing on debates within the PPGIS community around what is meant by "public" and "participatory." This chapter also documents projects that have employed tools to collect socio-spatial data, to illustrate the depth and breadth of on-going PPGIS activities and proposals to create a community of practice. The chapter culminates in a problem statement and the study's research question and objectives.

Chapter 3 explains the theoretical foundation that informs this study. General systems science is used to construct the study's design, organize themes that emerge, and visualize interrelationships. Structural contingency theory provides a framework in which to interpret insights derived from the themes as internal and external factors agency decision-makers are responding to in order to complete their task.

Chapter 4 justifies the rationale for selection of the case study for this research, the USFS. Important information about USFS organizational structure, the forest plan revision process and significant legislation are in this chapter that will assist the reader in contextualizing themes, excerpts and comments presented in the Findings and Recommendations chapters.

Chapter 5 and Chapter 6 detail the data collection and data analysis strategies. These chapters provide the reader with how participants were selected for this study as well as the iterative analytical steps applied in order to identify meta-themes within the dataset.

Chapter 7 presents the findings. This chapter explores the spectrum of sociospatial data and how it has been applied in forest planning. The salient themes are 
identified with selected text excerpts from participant responses. Each theme includes a brief summary with insights gleaned and compelling questions.

Chapter 8 is the conclusion with individual sections that focus on application of study findings. These include recommendations for practitioners, researchers, policymakers and educators. Limitations of the study are also included.

\subsection{Human Dimensions of Land-Use Planning}

Land-use management is the process of planning, executing and monitoring decisions affecting the development and use of physical spaces. Those tasked with making land-use decisions understand that these decisions are not made in a vacuum, but take place within a complex ecological, social, economic and political context. As Tuan (1977) notes, physical space transforms into "place" when it is imbued with special meaning and significance, both individually and shared. The values and meaning that humans associate with place at multiple spatial scales are central to individual and collective decisions about appropriate and desirable land uses. Williams and Stewart (1998) assert that the land-use planning effort is by and large "a public exercise in describing, contesting, and negotiating competing senses of place and ultimately working out a shared future sense of place" (Williams \& Stewart, 1998, p. 23). Lebel et al. (2006) add that to strive for sustainable management of natural resources in a world characterized by increasing uncertainty and change, effective strategies must be employed to deal with trade-offs and competing priorities among social, economic, and environmental objectives. Other researchers and practitioners echo these assertions, adding that a better understanding of both use- and value-based human-environment connections may help identify and ameliorate potential conflicts, increase trust and 
cooperation between land management agencies and the public, and reduce timeconsuming and expensive appeals and litigation of land management decisions (Allan et al., 2008; Brown \& Reed, 2009; Brown et al., 2010; Cheng et al., 2003; Nie, 2011).

Several U.S. government reports highlight the importance of understanding human dimensions of land-use planning. For example, the U.S. Department of Agriculture's (USDA) Forest Service Social Science Research Agenda (2004) specifies the following human-environment goals: (1) expand understanding of the human uses and values of natural resources and their implication for management; (2) develop information on the relationships among social, economic, and ecological sustainability; (3) gain knowledge about the role of community-based collaboration in public land management; (4) expand understanding of the human role in, and response to, environmental change; and (5) expand understanding of the links between human diversity and natural resource use. Researchers and practitioners propose that incorporating place-specific and values-centered data into land-use planning is a necessary endeavor in line with the USDA's social science imperative as doing so allows for geographically targeted land management strategies commensurate with user notions of sense of place and use preferences (Ban et al., 2013; Bennett et al., 2016; Brown \& Reed, 2009; Brown et al., 2020; Bryan et al., 2010; Cheng \& Daniels, 2003; Goodchild et al., 2000; Hall et al., 2009; Manfredo et al., 2014; Moon \& Blackman, 2014; Robinson et al., 2012).

The importance of understanding the human-environment connection appears in legislation as well. The U.S. National Environmental Policy Act (NEPA) of 1969, for example, contains a broad mandate for federal agencies to preserve and enhance the 
environment as trustees for current and future generations and requires that all federal agencies assess and report impacts of their activities from both an environmental and human welfare perspective. Its purpose is stated as follows:

To declare a national policy which will encourage productive and enjoyable harmony between man and his environment; to promote efforts which will prevent or eliminate damage to the environment and biosphere and stimulate the health and welfare of man; to enrich the understanding of the ecological systems and natural resources important to the Nation; and to establish a Council on Environmental Quality. (P.L. 91-190, 42 USC \$4321, Jan. 1, 1970)

At an administrative level, the U.S. Department of Agriculture's National Forest System Land Management Planning Rule dictates that the U.S. Forest Service, which manages over 193 million acres of public lands, solicits public input during the preparation or revision of comprehensive forest management plans. The agency must document not only how public interests and values have been collected, but also how this information has been integrated into the decisions and proposed actions that make up the plan (Federal Register, 36 CFR, Part 219, Subpart A §219.1(c)).

\subsection{The Evolution of Forest Planning, Sense of Place and Geospatial Technology}

The emphasis on the human dimensions of land-use planning in the U.S. today emerged from political and social debates around appropriate use of public lands, which often involved negotiating tensions between views around exploitation, conservation, and preservation. Barbier (2010), in his comprehensive study on economics and natural resource exploitation, points out that the rich and seemingly infinite supply of natural resources on the North American continent fueled European colonization and westward expansion and perpetuated an exploitive, profit-motivated mindset for several hundred years, vestiges of which still exist today. At the turn of the 20th century, however, new 
ways of thinking about conservation and preservation of natural resources gained traction. Gifford Pinchot, the first Chief of the U.S. Forest Service (established in 1905), is credited with formulating what is considered the modern view of natural resource conservation - the "wise use of the earth and its resources for the lasting good of men" (Pinchot, 1947, commemorative edition). Often in opposition to preservationists of the time, such as John Muir who extolled the intrinsic value of landscapes and rejected any sort of commodity-based management, Pinchot saw conservation as a means of managing the nation's natural resources for long-term sustainable commercial use. His concepts around multiple-use and sustainable practices are embedded in the mission of the U.S. Forest Service today.

Political and social events of the last decades of the 20th century provided impetus for another significant shift in the priorities of public lands management. As Hays (2009) explains, in the decades following 1970, major changes in the administrative structure and operational priorities of the U.S. Forest Service occurred as the agency responded to changing attitudes and demands from the polity at large. Broad public opinions around resource preservation, wildlife and watershed protection, recreation, and natural aesthetics, which had historically been subordinated to grazing and wood production, reflected growing environmental awareness and advocacy within the citizenry. This occurred in tandem with an emerging ecological paradigm around forest management proposed by the scientific community. The term "ecosystem management" was formalized in 1992 by F. Dale Robertson, then Chief of the USFS. Robertson stated, "[b]y ecosystem management, we mean an ecological approach... [that] must blend the needs of people and environmental values in such a way that the National Forests and 
Grasslands represent diverse, healthy, productive and sustainable ecosystems"

(Kaufmann et al., 1994, p. 1). On the legislative front, the National Environmental Policy Act (NEPA, 1969), the Endangered Species Act (ESA, 1973), the Wilderness Act (1964), and the National Forest Management Act (NFMA, 1976), among other environmental legislation, significantly increased the breadth and complexity of public land management priorities and objectives. Key provisions, particularly in NEPA and the NFMA, provide avenues for the public, as well as organized industry and environmental interest groups, to be involved in or to contest land management decisions.

Scholarship added a nuanced exploration of the psychology of place to this discourse. During this time that Yi-Fu Tuan introduced the term "sense of place" and explored its meaning in his seminal works: Topophilia (1974) and Space and Place (1977). Tuan focused on senses that enable us to have feelings for space and how this experience creates meaning and value around special places. Other scholars at the time, particularly in environmental psychology, explored ideas of place attachment (Altman \& Low, 1992; Stedman, 2003) and place identity (Proshansky et al., 1983). In short, place attachment is an affective bond that people establish with specific places where they feel comfortable and safe. Place identity is defined as a component of personal identity where people describe themselves in terms of belonging to a specific place.

At the turn of the 21 st century, these four trends propelled heightened interest in human-environment connections related to management of public lands - changing public attitudes and values around appropriate use of natural resources; environmental legislation that transformed public agency priorities and operations; application of the ecosystem management framework; and a plethora of academic research around sense of 
place, place attachment and place identity. As Hays (2009) points out, this confluence required land management agencies to rethink their operating protocols and decisionmaking processes. Within the USFS, practitioners and scientists began advocating for the inclusion of public values, particularly related to sense of place, into formal forest planning. Mitchell et al. (1993), Williams and Stewart (1998) and Kruger and Jakes (2003) published articles in forestry journals outlining the rationale for including emotional attachments and public attitudes that are spatially dependent into land management decisions. Several USFS general technical reports were also published shortly thereafter offering a research synthesis and new approaches to integrating sense of place into the planning process (Allen et al., 2009; Bright et al., 2003; Farnum et al., 2005; Galliano \& Loeffler, 1999; Hall et al., 2009; Kruger \& Williams, 2007). Willliams and Stewart (1998) sum up emerging views as follows:

By initiating a discussion about sense of place, managers can build a working relationship with citizens that reflects the complex web of lifestyles, meanings, and social relations endemic to a place or resource. Sense of place can be the shared language that eases discussions of salient issues and problems and that affirms the principles underlying ecosystem management. (Williams \& Stewart, $1998, p .18)$

\section{Public Participatory Geographic Information Systems. Geographic}

information systems (GIS) is a digital technology used to create, analyze, store, and visualize place-based data. GIS was first developed in Canada in the early 1960s to inventory its natural resources and create computerized layers of biophysical data. The use of GIS in land-use planning (and numerous other public and private sectors as well) has grown exponentially since the 1960 s in concert with increasing computing power and sophistication of data collection, analysis and visualization software (Sieber, 2006). 
As a student of human geography in the early 2000 s, with a focus on GIS technology, I was drawn to how GIS might elucidate important human-environment connections, improve collaboration between public agencies and stakeholders, and thus lead to better informed and less contentious land-use decisions. It was also at this time that a new term entered the scholarly literature - public participatory geographic information systems (PPGIS). PPGIS refers to the use of GIS digital technology to broaden public involvement in policy-making and elevate the interests and goals of nongovernmental entities, grassroots and indigenous groups, community-based organizations, and the public at large. PPGIS, particularly within the context of public agency processes, has the potential to engage stakeholders at various stages of collaborative planning. Uses of PPGIS include disseminating information on land-use proposals and analyses through spatial visualization (a.k.a. maps) and gathering public input on management strategies using mapping interfaces (Sieber, 2006).

Within the PPGIS world, human ecology mapping (HEM) refers to a broad set of PPGIS mapping strategies and tools that seek to spatially visualize the diverse and complex connections between humans and landscapes. Sense of place mapping is a subset of HEM that focuses on the values and meanings that people ascribe to particular landscapes, special places, and activities associated with those places and has particular utility in natural resource planning. Sense of place mapping produces socio-spatial data that can show hotspots of high use and value (e.g. overlooks or vistas) or where potential conflicting values and uses might occur (e.g. hunting versus foraging). (See McLain et al., 2013, for a comprehensive description of human ecology mapping perspectives, tools and analytical strategies). 
The utility of socio-spatial data lies in capturing sense of place information not part of standard demographic or economic databases (e.g. U.S. Census data). For example, a county-level map that visualizes economic activity may show that several counties are experiencing financial hardship due to falling revenues from timber harvests, leading to a decision to open up more forest land for harvesting. An approach that collects socio-spatial data from invested constituencies is likely to show a strong economic interest as well, but may also reveal the presence of potentially conflicting values or desired uses for specific places in the forest, such as an oft-used fishing spot or an area with particular historical or spiritual relevance. Gathering socio-spatial data allows land managers to better understand public use of the landscape and develop action plans that reflect the "shared sense of place" that Williams and Stewart (1998) describe. Black and Liljeblad (2008) sum up the advantages of incorporating socio-spatial data into natural resource planning as follows:

...embedded in the concept of place is a story about ecological function and appropriateness of human actions. Only by articulating these stories can we begin to illuminate the fundamental basis of conflict over appropriate land management practices. By placing these stories in an objective, generalizable framework, we can provide managers and the interested public the means to develop a deeper understanding of their relationships to place and thus, the opportunity to co-create new stories that may be even more appropriate for guiding behavior (both as federal managers and private individuals) in their current and future landscapes. (Black \& Liljeblad, 2008, p. 1)

Summary. The discussion above provides a historical account of four trends that culminated in a growing interest within land management agencies around the values people attach to places: (1) changing public attitudes and relationships with public lands, (2) environmental legislation with directives to engage the public in land-use decisions; (3) adoption of an ecosystem services paradigm that recognizes the non-monetary social 
and cultural value of public lands; and (4) sense of place as an important concept in understanding the values people attach to places. Public participatory GIS evolved concurrently with these trends and has been promoted as a tool to capture values-based spatial data that can inform land-use decisions.

The confluence of these four trends and advancements in PPGIS technology would seem to be the perfect recipe for productive public engagement and collection of socio-spatial data to aid in stewardship of public lands. But, as is usually the case, reality is a bit more complicated. The literature review that follows highlights two areas of research and scholarly interest that relate to this study's focus of inquiry - the use of socio-spatial data in forest planning. The first section briefly introduces ongoing debate within the PPGIS world around what meanings are being attached to "public" and "participatory" and questions on whether PPGIS is living up to its claims. The second section examines PPGIS case studies. The scholarly debates and research gaps discussed in these two sections provide the rationale for this study's research question and objectives that conclude the chapter. 


\section{LITERATURE REVIEW}

The literature on PPGIS is vast, encompassing numerous public and private sectors. I briefly discuss below two strands of PPGIS literature pertinent to this research: (1) debates around how various "publics" are represented in PPGIS practice and the significance of their "participation;" and (2) case studies using HEM strategies and tools. These strands certainly do not cover the depth and breadth of the PPGIS literature. They are selected to provide a scholarly foundation and rationale for development of the research question and objectives for this study.

\subsection{Public, Participation and PPGIS}

As soon as public participatory GIS became mainstream, scholars have been discussing the meanings and implications of "public" and "participatory" within the field of PPGIS. The questions posed are broad. Does PPGIS represent a democratization of knowledge production or is this an illusion (Dunn, 2007; Radil \& Anderson, 2018)? Who owns the data produced by PPGIS and how does that affect the relationship between agencies and the public (Elwood, 2006)? Which "publics" are included or excluded in decision-making that incorporates socio-spatial data? Does PPGIS lead to enhanced public participation and, if so, who is at the table and how are they influencing decisions (Kahila-Tani et al., 2016; Klein et al., 2019; McCall \& Dunn, 2012; Pfeffer et al., 2012)? Can PPGIS be an effective tool to enhance co-learning and collaboration between natural resource managers and stakeholders (Chen \& Mattor, 2010; McDonagh \& Tuulentie, 2020). Can PPGIS enhance civic engagement and agency? If so, what does that look like (Kotus \& Rzeszewski, 2020)? 
These are important questions with no singular answers (see Sieber, 2006, for a comprehensive, and still relevant, compilation and discussion of these topics). This lively discourse is an on-going call for researchers and practitioners to be cognizant and reflective around important issues of accountability, empowerment, control and appropriate use of socio-spatial data collected using PPGIS tools. These questions are on the minds of study participants as well and are highlighted where appropriate in the discussion of salient themes that emerge from the study's dataset.

In response to the debate described above, and as use of PPGIS has become more ubiquitous in the planning sphere, scholars have attempted to unify PPGIS into a community of practice built around shared principals that can provide researchers and practitioners with guidance and structure. Fagerholm et al. (2021), offer a PPGIS framework that categorizes data analysis and application into three phases: explain, explore and predict/model. During each phase, the socio-spatial data is interpreted, validated and applied in practice in different ways. The explore phase involves descriptive analysis and simple visualization of socio-spatial data. The goal of the exploration phase is to understand the data within its isolated context. The explain phase takes the analysis further and creates "new knowledge" by comparing socio-spatial data with other geographic data, e.g. integrating data layers showing special places with a recreation opportunity spectrum map and making inferences about public acceptance of proposed recreation sites. Identifying spatial patterns (e.g. clustering or dispersion) are also part of this analysis phase and can be used to determine hotspots of high use for different land use categories. The author's final phase, predict/model, includes tools to generalize the spatial analysis to other contexts, such as making correlations between 
public landscape values and physical landscape characteristics. This is the most advanced phase which requires skills and experience in applying GIS spatial visualization and quantitative analysis and modeling tools.

While this article primarily focuses on data collection and validation challenges and appropriate spatial analysis techniques at each phase, a topic that is well beyond the scope of this study, it nonetheless provides context and language in which to discuss study findings around use of socio-spatial data. Each phase represents a specific purpose for collecting socio-spatial data with different requisite skills and experience required to interpret and integrate the data. In particular, the purpose assigned to the collection of socio-spatial data and its relationship with resources that may (or may not) have been available is an important consideration that will be explored in Chapter 7.

\subsection{PPGIS Strategies and Case Studies}

Case studies using HEM tools for capturing and spatially displaying socio-spatial data have been reported in academic journals and land management agency reports for more than two decades. One of the earliest published case study dates to the year 1998 (Brown \& Reed, 2000) and documents a mapping study on the Chugach National Forest in Alaska. Since then, HEM projects in natural resource planning have greatly expanded and include studies in North America (Alessa et al, 2008; Brown \& Raymond, 2007; Hall et al., 2009; McLain et al., 2013; Nielsen-Pincus, 2011; Sherrouse et al., 2011), Finland (Kangas et al., 2008; Tyrvainen et al., 2007), Wales, UK (Ruiz-Frau et al., 2011), Australia (Bryan et al. 2010; Raymond \& Brown, 2006), China (Zhang et al., 2019; Zhau, et al., 2019), Africa (Fagerholm \& Kayhko, 2009; Fagerholm et al., 2019) and South America (Blake et al., 2017). 
Generally, human ecology mapping case studies provide detail on the mapping methods used, the characteristics of the data collected, and make suggestions on how the data might be used in planning. Though there are numerous case studies in the literature, published articles rarely report how the data was actually used in making planning decisions, with some exceptions (Biedenweg et al., 2014; Jankowski et al., 2021; Rose et al., 2020; Tyrvainen et al., 2007). This apparent lack of published documentation has led numerous authors to suggest that socio-spatial data have not been applied effectively in land-use decisions, despite documentation of the efficacy of HEM strategies and the data's potential to improve public participation in and/or acceptance of land-use decisions (Ambrose-Oji \& Pagella, 2012; Bennett et al., 2016; Brown \& Donovan, 2014; Brown et al., 2020).

Numerous possible explanations have been proposed. Brown and Fagerholm (2015), in their analysis of 30 published case studies, focus on the nature of the data and suggest that the lack of standardized best practices in data collection methods and the qualitative nature of the data itself call into question its suitability for decision-making. Other authors suggest structural barriers such as limited staff capacity, insufficient budgets and lack of leadership support (Robinson et al., 2012). Organizational culture has also been proposed as a possible explanation, such as the presence of a culture of risk aversion (Allan et al., 2008; Haynes, 2005), professional siloing between the social and natural sciences that hinders interdisciplinary collaboration (Anstedt, 2010; Barbour, 2007; Biedenweg \& Nelson, 2017), and devaluing public input in planning processes (Brown et al., 2020). And, finally, some authors point to external factors embedded in legislation and directives, such as the exclusive focus on quantitative data that formally 
defines Best Available Scientific Information (BASI) in planning protocols (Charnley et al., 2017) and concerns that socio-spatial data and the methods used to collect it are not appropriate or legally defensible within the context of NEPA compliance (Hoover \& Stern, 2014; Kaiser, 2006).

\subsection{Problem Statement and Research Question}

Having worked with a team from the U.S. Forest Service and Portland State University (Portland, OR) in the field of sense of place mapping, I also experienced this dissonance between agency acknowledgement of the value of socio-spatial data and what I interpreted, at the time, as reticence among practitioners around gathering this data and incorporating it into land-use planning. I had originally entertained a research topic focused on participatory and policy implications of embedding socio-spatial data into the forest planning process, using case studies from the team's HEM mapping work in national forests in Washington State. I quickly realized that such a study was premature and I needed to take a step - or two - back. There were dynamics at play suggesting barriers and challenges around the use of GIS-based tools to integrate socio-spatial data in land-use planning, despite recognition of the potential benefits. In addition, the USFS at the time was deeply engaged in updating comprehensive forest management plans under a newly registered forest planning rule that seemed, on its face, to elevate public participatory processes and acknowledge the non-monetary, values-based services national forests provide. It presented a unique opportunity to apply a broader inquiry on how socio-spatial data was (or was not) used within a forest planning process at a time in which on-the-ground forest planners were highly focused on collecting data to inform their plan revisions. 
This study starts with the basic assumption that understanding public values and land-use preferences is not only a desired component of federal land management, but also integrated into laws and directives that govern the process. Such a combination should, ostensibly, provide strong incentives for land management agencies to adopt technology tools and processes that integrate socio-spatial data into planning decisions. Despite numerous propositions on what might explain the apparent reticence, there are no empirical studies to date that seek to gather primary data from scientists and on-theground practitioners focusing exclusively on how socio-spatial data is collected and incorporated into a natural resource planning process. This type of exploratory research is a necessary step to answer the basic question - what are factors that inhibit or encourage integration of socio-spatial data into land-use planning? Results from such a study will identify the barriers as they are experienced by those involved in forest planning and to rethink operational and decision processes in order to mitigate challenges and maximize the benefits the data might offer.

Using the USFS as a case study, this research focuses on identifying significant factors that influence the collection and integration of socio-spatial data in national forest planning. Using an inductive approach, themes are developed from semi-structured interviews with USFS practitioners and scientists involved in forest planning. The broad research question reflects the exploratory nature of the research topic and is stated below.

Research Question: What factors represent significant barriers or opportunities in the collection and integration of socio-spatial data in national forest planning?

The objectives of the research are to: 
1. Identify factors within the USFS that represent barriers, challenges or opportunities in collecting and using social science and socio-spatial data in national forest planning within four broad topical areas - structural, perceptual, cultural and external.

2. Assess the relative significance of and relationships between the identified factors through applied thematic analysis.

3. Assess differences in the identified factors within the agency's administrative units - national forests, regional offices and research stations.

4. Refine an initial conceptual model to inform practitioners and suggest practical opportunities and areas for further research.

Summary. The discussion thus far has highlighted the following: (1) sense of place is important in land-use planning to capture human-environment connections; (2) the need to understand and incorporate socio-spatial data into land management decisions is embedded in legislation and administrative directives; and (3) tools and strategies to capture socio-spatial data exist, but there is little formal documentation of the extent to which this type of data are integrated into forest planning decisions. The research question seeks to provide this documentation and garner insights on the challenges and opportunities expressed by USFS personnel that are deeply involved in engaging the public during the process of revising forest management plans. With the research question and objectives in mind, Chapter 3 describes the theoretical foundation and the initial conceptual scheme that provide the structural framework for the study design and analytical framework for interpreting the results. 


\section{THEORETICAL FOUNDATION}

In broadest terms, this study seeks to synthesize existing and acquired data to better understand the dynamics occurring around the use or non-use of socio-spatial data in forest planning within a complex organization. Since this study focuses on dynamics occurring during an intense organizational process (forest plan revision), the theoretical foundation is drawn from organization theory. General systems science is used to inform the study design, analysis and findings on a practical level. Structural contingency theory provides a framework in which to situate the findings and explore potential for future research.

\subsection{General Systems Theory and Systems Science}

Systems theory is uniquely suited to framing an exploratory research project, as it strives to make dynamics occurring within elements of a system visible and actionable. Systems science provides the framework for the study design and is used to interpret the findings on a practical level. This section provides a brief overview of systems theory, its key concepts and how it is applied in the context of this study.

General systems theory seeks to understand the broad properties of systems in a simplified, yet holistic, manner that captures a system's multidimensionality. Ludwig

von Betalanffy, an Austrian biologist, first proposed general systems theory as a means to reconcile the law of entropy in classical physics (that all matter will inevitably move from order to disorder) with his observations of the stabilizing proclivities of complex biological organisms (e.g. their ability to regulate body temperature). He noted that living systems somehow avoid the degradation expected in physical systems and argued this is due to the fact that organismic systems are not closed, but instead open and fluid. 
Their component parts are constantly interacting with each other, as well as the external environment, to achieve and maintain a state of equilibrium, later termed a stable state (von Betalanffy, 1938).

A system is an integrated whole whose properties cannot be fully explained by or reduced to its individual elements, aptly summed up by the aphorism "the whole is greater than the sum of its parts." Systems theory perceives the world in terms of boundaries and relationships, asserting that the properties of systems can be derived from the interactions of its parts (von Betalanffy, 1972). The foundation of systems theory is the proposition that all types of systems have characteristics in common that can be used to identify, classify or describe the arrangement of the parts and reciprocal mechanisms that link them. In this way, general systems theory combines knowledge of system structure, relationships between elements that make up that structure, and flows of information and resources between the elements (Skyttner, 2015). What binds these components into a system is how they work together to serve a purpose or achieve a goal (Arnold \& Wage, 2016). For example, forest planning is a system (within a larger system) in that it contains numerous organizational elements (staffing, laws and directives, standard operating procedures), mechanisms that connect these elements (communication pathways, decision-making hierarchies), and flows of information and resources (best available science, funding streams). What binds forest planning as a system is its ultimate goal to produce a comprehensive forest management plan.

The goal of systems science, then, is to model a system's dynamics, constraints and conditions in order to discover core principles or axioms. Over time, general systems theory has matured and developed into more specific systems-based theories, such as 
chaos theory. According to Wheatley (2006), chaos and order are not simply mirror images of each other, but inextricably connected. Chaos is necessary to produce new creative ordering. For example, a system reacting to change or uncertainty may look chaotic and unpredictable, but in "that state of chaos, the system is held within boundaries that were well-ordered and predictable. Without the partnering of these two great forces, no change or progress is possible" (Wheatley, 2006, p. 13). It is useful to identify the boundaries that serve to contain what may be perceived as chaos or uncertainty in order to understand and improve system performance.

A common criticism of systems theory is that it is too general and not really a theory at all (Johnson, 2019). However, proponents of systems theory are quick to point out that it is not intended to be a general theory of everything and thus potentially useful for nothing (von Betalanffy, 1972). Instead, systems theory strives to provide a language or conceptual lens for thinking and talking about complex, dynamic, holistic systems, particularly by combining the knowledge of diverse disciplines to apply an integrative approach to a topic, issue or challenge. The value of systems theory lies in enhancing explanatory power and prediction of observed phenomena through a holistic epistemology (Adams, 2012; Johnson, 2019; Skyttner, 2015).

The key concepts in systems theory provide an epistemic framework and language in which to interpret and explain systems and, in relation to the general topic of interest in this study, what constitutes barriers and opportunities within a system. A sampling of key concepts within systems theory include:

Hierarchy: The more complex the organism, the more it will contain a nested set of systems within systems that together represent a whole. 
Reciprocal Transactions: The linear, circular or cyclical exchanges that systems engage in and how they influence each other; related to Chains (Chain Reaction or Causal Chains) which is the extent to which an external input into the system or a change within the system reverberates and affects other elements.

Feedback Loop: The process by which elements within the system react to other elements, either positively or negatively.

Adaptation: The tendency of a system to make internal changes deemed necessary to keep fulfilling its core purpose.

Since this study is exploratory, systems theory is not used to develop and test a hypothesis around generalized system properties. The goal of the study is to take an observed phenomenon and place it within a broader systemic context to better understand the interrelationships of various elements within the system. This is the goal of systems science. The holistic lens and language described above inform this study's design, the conceptual framework, choice of analytical tools, and practical application of the results. The following bullet points describe specific areas in which systems science has been incorporated into this study.

- Study goals focus on the links and relationships between organizational elements and how they function together.

- The initial conceptual framework that identifies organizational factors that may represent barriers and opportunities is extracted from numerous academic disciplines.

- The conceptual framework reflects a systems approach by highlighting not just the elements at play in the system, but also the relationships between the elements.

- The study incorporates targeted, but also open-ended, data collection methods that are grounded in gathering on-the ground knowledge and experience of scientists and practitioners deeply involved in the topic of study. Insight is mined from the data itself, the whole "story" as revealed by study participants that represent different functional units and areas of expertise within the organization. 
- The open-ended aspects of the data collection protocols allow participants to answer general prompting questions in a holistic manner that encourages sharing insight in a systemic way, as opposed to survey questions that target specific organizational elements or factors.

- No specific propositions or predictions are assumed in the development of the thematic coding structure or in analysis and interpretation of the textual data. Thematic analysis tools are selected and applied in order to group text into common themes; thematic networking is used to create visuals of how themes are grouped and linked to assist in interpreting the results within a systems framework.

The primary purpose of using a systems science approach is to refine an initial conceptual framework in a way that visualizes interdependencies revealed by themes derived from the data. The study protocols and analytical strategies are designed to thematize multiple organizational factors - structures, functions and external constraints - that interact with each other within an interconnected system. Using a thematic map of this system provides a tool to identify and interpret the impacts of barriers, challenges and potential opportunities on the ultimate performance measure - the extent to which socio-spatial data is incorporated into a forest management plan. Practical applications can be discussed within the context of the system as well, such as exploring the efficacy or potential impact of ideas explicitly or implicitly expressed by study participants.

\subsection{Structural Contingency Theory}

Contingency theory examines organizational elements to yield insights about constraints and opportunities that influence organizational structure, behavior and performance outcomes (Donaldson, 2001). Contingency theory, as a unique field, emerged from earlier work in organizational theory. Emery \& Trist (1965), for example, introduced the concept of the "causal texture of the environment" (Emery \& Trist, 1965, p. 22). Elements within an organization interact internally and those elements also 
interact with the environment. However, there are also processes occurring within the environment itself that influence these exchanges. More importantly, as the authors point out, "in considering environmental interdependencies...the laws connecting parts of the environment to each other are often incommensurate with those connecting parts of the organization to each other, or even with those which govern the exchanges" (Emery \& Trist, 1965, p. 22). In this sense, Emery and Trist add the environment as an independent actor that can significantly influence organizational structure and behavior. This dynamic is evident in the preceding discussion on the connections between changing public sentiments around environmental issues that helped foster a plethora of environmental legislation which in turn significantly impacted public land management agency operations and priorities.

During this period, J. D. Thompson published Organizations in Action: Social Science Bases of Administrative Theory (1967). Thompson made explicit links between environmental conditions and organizational features and developed a consolidated framework in which to construct numerous testable hypotheses. Thompson's framework builds upon the assertion that different organizational forms are a direct reflection of an organization's response to various contingencies, such as available technology, organizational goals, environmental pressures, and challenges in coordination and communication. This framework was used to build theory and stimulate research and is considered by many scholars to be the founding work in contingency theory.

That organizations develop certain structural forms in response to various internal and external forces, or contingencies, is a topic taken up by numerous other researchers. Mintzberg (1983), for example, translates concepts of organizational structure into 
graphical figures representing five organizational design configurations. Mintzberg's framework illustrates the importance of the different roles within an organization (e.g. strategic planning; operating core; support staff) and coordinating mechanisms (e.g. task assignments, decision hierarchies). How these structural features of an organizations are combined, in the context of the environmental factors in which it must operate, determines its configuration. Since these initial scholarly works, contingency theory has been applied in research focusing on numerous topics within organizational science, including management control mechanisms (Felicio et al., 2021; Fisher, 1998), organizational adaptability (Jennings \& Seaman, 1994; Battilana \& Casciaro, 2012), leadership (Fielder, 1967; Suharyanto \& Dwi Lestari, 2020), organizational knowledge production (Birkinshaw et al., 2002), and many others.

While a quantitative study based on contingency theory is not presented here, the constructs and insights provide a theoretical lens in which to interpret the results of this study and make suggestions for further research. Structural contingency theory, which focuses on the formalized components of an organization, dovetails with systems science. Systems science seeks to identify critical organizational factors and to understand the flows and feedback loops between them in order to improve organizational performance. Structural contingency theory seeks to establish causal relationships between elements of internal organizational structure (such as a particular form of decision-making) and external contingencies (such as relevant laws) that the organization must navigate and how this interaction affects performance. Contingencies, in this context, are internal and external factors the organization is responding and adjusting to in its efforts to achieve optimal performance (Donaldson, 2001). Internal contingencies refer to formal 
organizational structures such as decision hierarchies, standard operating procedures, and division of labor. External, or environmental, contingencies are factors outside of the organization, beyond its ability to directly control, that affect organizational functions. These include social, cultural, economic, political and environmental factors that may lead to rigidity, uncertainty or complexity in the environment in which the organization operates (such as legislation, political proclivities, market forces, or even the probability of natural disasters).

The hypothetical questions posed by contingency theorists revolve around the extent of congruence between internal and external contingencies. Do the structural elements of an organization "fit" the external environment in a way that maximizes organizational effectiveness? Figure 1 displays this relationship between organizational structures and congruence posited in contingency theory.

Figure 1. The role of congruence in structural contingency theory.

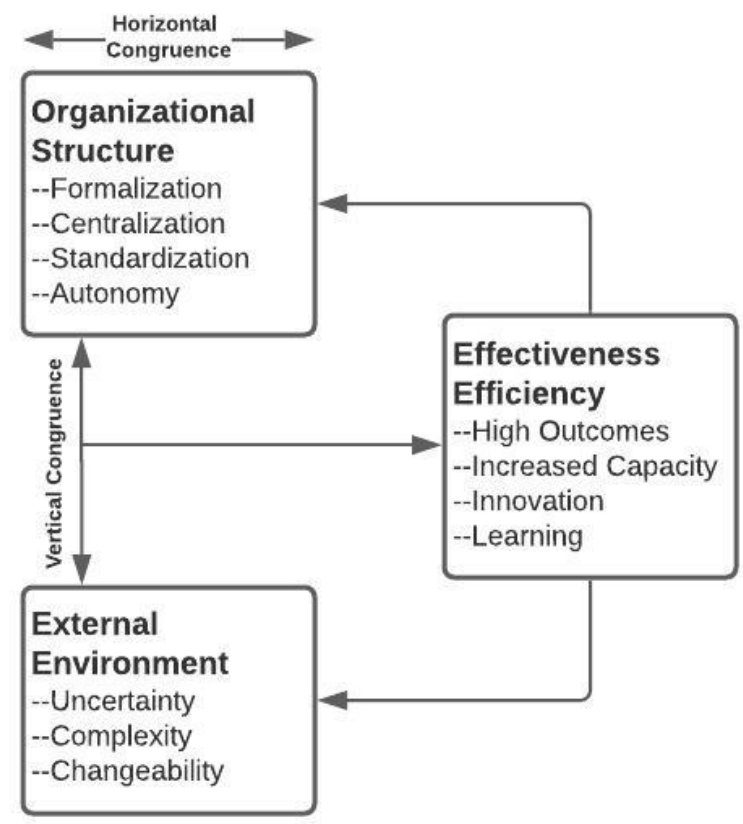

Source: Adapted from Pennings, J. M., 1998. 
The left side of Figure 1 shows a set of internal structural elements and a set of external environmental dimensions. Each element can be envisioned on a spectrum, for example decentralized to centralized decision-making and rigid to changeable environmental conditions. The foundation of structural contingency theory is the concept of "best fit" (Drazin \& Van de Ven, 1985; Pennings, 1998; Volberda et al., 2012). When applying structural contingency theory, the structures within organizations and the environmental factors the organization encounters are operationalized, identified and then placed within these spectrums. The extent of congruence (or fit) can be analyzed by comparing a structural element with an environmental element and hypothesizing about whether this is a good fit or not as it relates to a performance measure. Vertical congruence, or external fit, requires that the practices and strategies of the organization match the environmental conditions faced by the organization in order for it to achieve a performance objective. Horizontal congruence, or internal fit, is attained when internal practices, strategies and structures within the organization work together to deliver a desired outcome (Kathuria et al., 2007). So, a general hypothesis may state that an organization with a highly centralized decision process, that must also deal with a highly changeable external environment, will result in poor performance, and then testing to determine whether rigid decision hierarchies can sufficiently adapt to changing external conditions and the extent to which that (in)congruence affects performance.

Contingency theory also posits that organizations are compelled to adapt over time in response to changing contingencies in order to maintain efficiency and effectiveness. This is represented by the returning arrows between the structure and 
effectiveness/efficiency boxes. Contingency theory seeks to understand how these adaptations occur and their effects on organizational performance. The theory postulates that organizations that are able to recognize dysfunction within elements of organizational structure and modify them to enhance congruence horizontally and vertically are more likely to be successful in the long-term than those who do not.

A common criticism of contingency theory is its tendency toward reductionism. As Drazin and Van de Ven (1985) point out, studies using structural contingency theory tend to focus on how single environmental factors affect single structural characteristics and how they interact to explain a single measure of performance. The authors argue that applying a systems approach to contingency theory allows a researcher to explore the many contingencies, structural features, and performance criteria present within the organization as well as the interdependencies between them. Applying a systems philosophy also allows incorporation of the general systems theory concept of equifinality. As originally defined by Ludwig von Bertalanffy (1968), equifinality is a general property of open systems such that "... as far as they attain a steady state, this state can be reached from different initial conditions and in different ways; it is thus equifinal!" (von Bertalanffy, 1968, p. 79). This allows for interpreting best fit within structural contingency theory as "feasible sets of equally effective alternative designs, with each design internally consistent in its structural pattern and with each set matched to a configuration of contingencies facing the organization" (Drazin \& Van de Ven, 1985, p. 520).

For the purpose of this study, equifinality will be highlighted to assist in examining study findings and informing suggestions for further research. As mentioned 
above, equifinality is one avenue of research, combining systems theory and contingency theory, that is particularly relevant to this study. Gresov and Drazin's (1997) important contribution to a systems approach to structural contingency theory states that that "an organization will perform effectively if the critical functions it must carry out, as determined by the environment, are met by its organizational structures" (Gresov \& Drazin, 1997, p. 408, emphasis added). The external environment determines the ultimate function of an organization (e.g. to produce a product or service that meets a demand), but not what structures the organization chooses to incorporate into its organizational design to produce that product. Any particular structure may address multiple functions and any particular function may be fulfilled by alternative structures or processes. Functions also determine the performance outcome objectives. For example, one function of the USFS is to produce a comprehensive forest management plan (function) in compliance with applicable law (environment). However, the organizational structures in place (such as decision processes, communication pathways, staffing strategies, task allocations, funding streams, etc.) may be employed in various ways and to varying degrees to accommodate the function. Gresov and Drazin (1997) propose a matrix in which to assess levels of structural and functional equifinality by comparing the functional demands imposed by the external environment on the organization and the latitude available to managers and decision-makers to modify organizational structures to address these demands. Figure 2 shows this classification matrix. 
Figure 2: A classification matrix of equifinality scenarios.

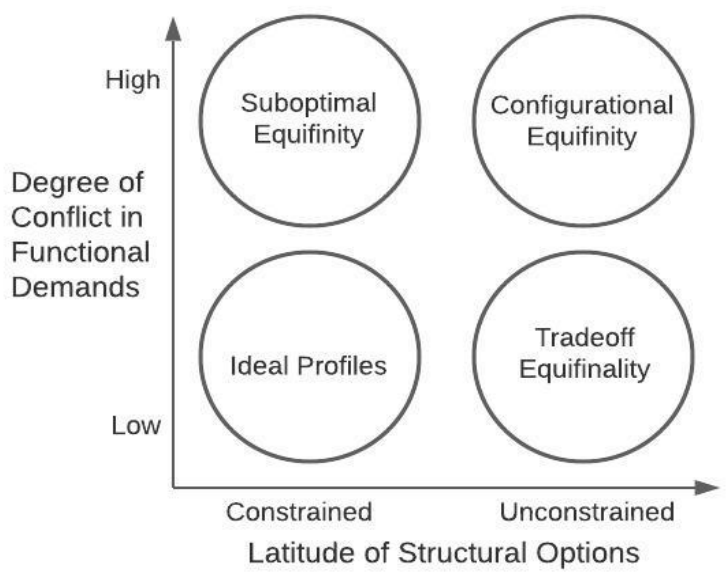

Source: Gresov \& Drazin, 1997.

The vertical axis in Figure 2 differentiates organizations by the extent to which the organization must balance multiple and/or conflicting functions. The horizontal axis indicates the degree to which managers have latitude or control over the structures embedded within the organization to fulfill these functions. The circles within the matrix represent four possible scenarios along these axes.

Ideal Profiles: This profile represents low functional conflict with fixed structures. This includes organizations that have a singular or highly dominant function and fixed structures that have been specifically designed to accomplish that function. As the structures represent a best fit, there is little conflict or incentive to adapt in this scenario unless environmental contingencies change significantly.

Suboptimal Equifinality: This scenario is characterized by multiple and/or conflicting functions coupled with rather fixed structures. Organizations in this category face a conundrum. Because there is limited ability to modify or develop new structures to meet different kinds of functional demands, to accomplish one function means that other functions must be subordinated and are likely to be suboptimal in their outcomes. 
Configurational Equifinality: This scenario is characterized by multiple and conflicting functional demands yet the structural options available to the organization are relatively unconstrained. In this situation there are simultaneous tradeoffs available in matching multiple functions and structures. Under these conditions, organizations will attempt to minimize functional conflicts by establishing flexible internal structures that will meet most of the functional demands. This will result in a number of organizational design profiles that perform reasonably well among all functions.

Tradeoff Equifinality: This scenario reflects a singular or dominant organizational function. However, many structural alternatives are available to meet this function. This allows managers flexibility in choosing which structures should be incorporated into the organizational design to accommodate the functional need and may do so based on knowledge of various tradeoffs, or even personal preference for different structural components. In this scenario, it is likely that organizations with the same function will manifest different, yet equally effective, structures.

Concepts within systems science and contingency theory provide a useful framework and language with which to situate study findings. A focus on connections between elements of organizational structures and processes, drawn from systems science, are used to construct an initial conceptual framework to guide the study design (described in the next section) as well as the construction of a thematic network derived from the dataset's salient themes (that will be presented in Chapter 7). Concepts of contingencies and congruence assist in interpreting study results as a set of internal and external contingencies that the organization is responding to. As a prelude to a more in- 
depth discussion of study methods, the following section outlines the initial conceptual framework that informs the study design.

\subsection{Initial Conceptual Framework}

This section covers the research approach and initial conceptual framework. I selected an inductive approach for this research as this approach is uniquely suited to addressing the exploratory nature of the research question and objectives. As Marshall and Rossman (2016) explain, exploratory research, as opposed to conclusive research, seeks to determine the fundamental nature of an observed phenomenon in order to inform and guide future inquiries and studies. The results of an exploratory study are not intended to provide definitive answers, but instead to identify a range of factors, causes, or relationships that provide strategic insight for both theory and practice.

Inductive research is a systematic process that draws generalized conclusions from specific data, guided by a set of research objectives. Inductive research is most appropriate for questions of a qualitative and multifaceted nature and seeks to (1) condense raw textual data into a brief summary format; (2) establish links between the research objectives and summary findings derived from the raw data; and (3) develop or modify a framework of factors, configurations and processes evident in the raw data (Thomas, 2006). The goal of the inductive approach is to remove restraints imposed by more prescribed methodologies in which key themes can often be obscured, reframed or left invisible because of preconceptions built into the data collection or analysis process. The following table lists the advantages and limitations of inductive research. Specific strategies to mitigate limitations of this approach are discussed in the data collection and analysis chapters within the specific context they are applied. 
Table 1: Advantages and limitations of the inductive research approach.

\begin{tabular}{|l|l|}
\hline \multicolumn{1}{|c|}{ Advantages of Inductive Research } & \multicolumn{1}{c|}{ Limitations of Inductive Research } \\
\hline $\begin{array}{l}\text { Ability to adapt, expand or modify the conceptual } \\
\text { framework and data collection strategies as } \\
\text { appropriate in response to new information. }\end{array}$ & $\begin{array}{l}\text { Qualitative data is often collected from a small } \\
\text { number of cases or samples which limits ability to } \\
\text { extrapolate findings to a broader level. }\end{array}$ \\
\hline $\begin{array}{l}\text { Reveals meaning and relationships in the data that } \\
\text { might be ignored or rendered invisible in more } \\
\text { structured research models. }\end{array}$ & $\begin{array}{l}\text { Can be time consuming and costly (transcriptions, } \\
\text { multiple rounds of coding). }\end{array}$ \\
\hline $\begin{array}{l}\text { Evaluative in nature which fosters the development } \\
\text { of improved strategies and processes at a } \\
\text { practitioner level based on cumulative insights. }\end{array}$ & $\begin{array}{l}\text { Difficulty parsing and interpreting often complex, } \\
\text { meandering, open-ended narrative data in a } \\
\text { systematic manner. }\end{array}$ \\
\hline $\begin{array}{l}\text { Informs areas and types of research worth pursuing } \\
\text { in the future, including theory-building. }\end{array}$ & $\begin{array}{l}\text { Potential researcher bias, particularly in } \\
\text { development and application of the coding scheme } \\
\text { for narrative data as well as interpretation of that } \\
\text { data (deciding what is important to note as opposed } \\
\text { to what is not). }\end{array}$ \\
\hline
\end{tabular}

Source: Adapted from Marshall \& Rossman, 2016.

The U.S. Forest Service (USFS) serves as the case study for this research. The case study is a common approach used in inductive research. This approach collects descriptive data from a single or small number of cases in order to develop conceptual categories, draw out patterns of interaction, or challenge assumptions (Marshall \& Rossman, 2016). According to Yin (2009), "the distinctive need for case studies arises out of the desire to understand complex social phenomena...the case study method allows investigators to retain the holistic and meaningful characteristics of real-life events" (Yin, 2009 , p. 4). In this sense, the case study is ideally suited to in-depth exploration of issues and developing insight through the experiences and views of the study participants themselves (Hartley, 2004; Stake, 2005).

The case study approach is flexible and adaptable. It can apply varying levels of analysis (individual, organization, social system), incorporate a wide variety of data collection techniques (documents, observations, interviews, surveys), and employ 
different analysis strategies (thematic, discursive, ethnographic). On the other hand, a common criticism of the case study method is that the results are not generalizable to a wider population or context. While this is certainly true in a statistical sense, Yin (2009) points out that the case study is not intended to represent a statistically valid sample of a population of interest. On the contrary, case studies are "generalizable to theoretical propositions and not to populations or universes" (Yin, 2009, p. 10).

Whatever research approach is selected, decisions need to be made about what kinds of data would be most appropriate to collect, how to collect the data, from whom, and the lens in which the results will be interpreted. Conceptual frameworks help to inform these decisions. A qualitative conceptual framework is often built from multidisciplinary bodies of knowledge or a synthesis of findings across qualitative studies (Miles \& Huberman, 1994).

While conceptual frameworks are not always used as a strategic tool to guide inductive research (e.g. grounded theory starts with a blank slate), when researchers and practitioners have already mused about, discussed and offered possible explanations around a topic of interest, as is the case in this study, the conceptual framework becomes a useful synthesizing tool. The conceptual framework is not immutable in inductive research. On the contrary, greater understanding of the phenomenon of interest through the final analysis and interpretation of the data is used to adjust, revise, realign or even totally reject the initial framework. Based on a general reading of academic literature on forest planning, I determined that possible factors influencing collection and uptake of socio-spatial data in planning fall into four general topical categories - structural, external, cultural and perceptual. The following figure illustrates these four categories. 
Figure 3: Schematic of the study's initial conceptual framework.

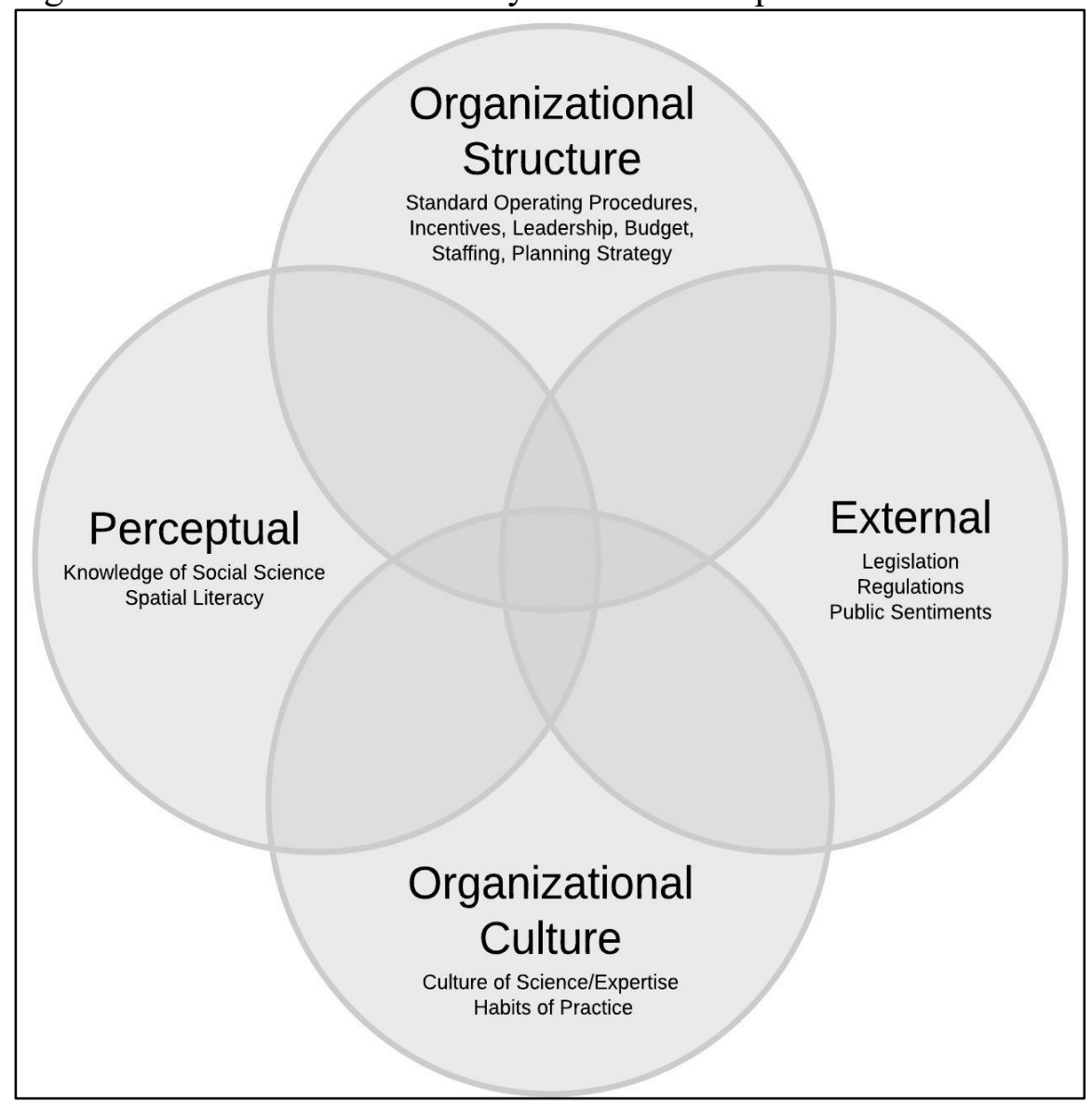

Figure 3 provides a visual representation of the initial conceptual framework adopted in this study. The framework identifies four general categories, a list of possible factors to consider within these categories, and illustrates the potential for linkages and relationships to occur between two, three or all four categories. Each category, and the factors within, were drawn from an interdisciplinary review of literature and are defined below.

1. Organizational Structure: Mintzberg (1983) defines the structure of an organization as “the sum total of the ways in which its [the organization's] labor is divided into distinct tasks and then its coordination is achieved among these tasks" (Mintzberg, 1983, p. 2). Structural factors include the organization's authority hierarchy, 
communication and decision protocols, standard operating procedures, performance measures (both for outcomes and employee incentives), and budgetary frameworks.

2. External: External factors refer to processes occurring outside the organizational structure and are often beyond the organization's direct control. Yet, these processes still significantly impact organization functions. In the case of a public agency, these factors include applicable law (e.g. NEPA), administrative rules (e.g. Office of Management \& Budget regulations around collection of public data) and public sentiments and expectations regarding appropriate land management practices.

3. Perceptual: USFS personnel, as is the case for many large agencies and organizations, represent a diversity of professional disciplines and skill-sets. One of the most basic obstacles to effective communication and collaboration is the differing conceptual lenses and analytical tools which different disciplines employ. Challenges exist in interdisciplinary decision-making because cross-disciplinary collaboration requires a significant investment in facilitation, funding and dedicated staff time to develop professional working relationships and foster mutual learning (Strang, 2009).

4. Organizational Culture: Schein (2010) defines an organization's culture as “a pattern of shared basic assumptions learned by a group as it solves problems of adaptation and internal integration, which has worked well enough to be considered valid and, therefore, to be taught to new members as the correct way to perceive, think, and feel in relation to those problems" (Schein, 2010, p. 18). An organization's culture is often reflected in its formal structure, through standard operating protocols. However, culture also refers to informal expectations, habits and patterns of behavior and thinking. 
Summary. These four categories are not intended to be exhaustive. They are used to compile and organize current thought on the study's research question and identify useful areas to focus on in the data collection instruments. Constructing the initial conceptual framework as a Venn diagram illustrates the study's goal to explore potential linkages between the factors. Interview protocols developed for the study reflect these initial categories. Prompting questions allow an open-ended discussion of the factors identified in each category. It is important to note that an initial conceptual design is informative only. The expectation is that it will be significantly refined to illuminate salient factors and relationships that emerge directly from the study's findings. The next chapter provides the rationale for selection of the case study, the means through which to apply the initial conceptual framework. Though brief, it provides the reader with basic information about USFS organizational structure, elements of the forest plan revision process, and laws that circumscribe agency functions. 


\section{CASE STUDY: U.S. Forest Service}

The unit of analysis in this case study is an organization. The case study is the U.S. Forest Service (USFS), a federal land management agency within the U.S. Department of Agriculture. The USFS has been selected as a case study for the following reasons:

1. The USFS is the largest land management agency in the U.S. federal system and is tasked with managing approximately 193 million acres of national forests and grasslands in the public interest (USFS Forests and Grasslands; Forests and Grasslands | US Forest Service (usda.gov)).

2. The USFS mission and governing law explicitly highlight the humanenvironment connection, which should encourage valuation of what socio-spatial data can contribute to land management planning decisions.

USFS Mission: Sustain the health, diversity, and productivity of the Nation's forests and grasslands to meet the needs of present and future generations (USFS Mission: https://www.fs.usda.gov/about-agency).

The Multiple Use-Sustained Yield Act of 1960 (MUSYA) is the primary statute that establishes the current public mission of the USFS. Multiple use, in MUSYA, means the "management of all the various renewable surface resources of the national forests so that they are utilized in the combination that will best meet the needs of the American people." Sustained yield refers to the "achievement and maintenance in perpetuity of a high-level annual or regular periodic output of the various renewable resources of the national forest without impairment of the 
productivity of the land" (Multiple Use Sustained Yield Act of 1960, 16 U.S.C. $\S \S 528-531)$.

3. The USFS is required by law (National Forest Management Act of 1976, 16 U.S.C. $\S \S 472 a, 1600-1606)$ to produce comprehensive forest management plans on a regular basis with significant public input. Many of these plans have recently been completed or are in the process of revision under a new forest planning rule, providing a rich source of study participants deeply immersed in the land-use planning process.

The National Forest Management Act of 1976 (NFMA) dictates the administrative rules that govern USFS activities, primarily by requiring preparation of comprehensive forest management plans for all national forests. The Forest Management Planning Rule (77 F.R. 21260) stipulates the process under which forest management plans are to be created. In March of 2012, the U.S. Department of Agriculture submitted a notice in the Federal Register outlining a new planning rule to replace an old 1982 rule. This new planning rule represents a departure from historical planning processes dominated by the biophysical sciences and organized into relatively discrete management objectives (e.g. clean water, species protection, recreation zones, timber output, etc.). Instead, the new planning rule requires identifying management priorities using a more holistic ecosystems approach that recognizes human-environment connections. Of note, the new planning rule specifically mandates that cultural services (the non-material benefits people receive from the forests, such as aesthetic, spiritual and heritage values) be explicitly incorporated into forest plans and that this information be collected using an 
extensive and inclusive public participatory process. This public charge should, ostensibly, provide a significant incentive for the agency to consider collecting and incorporating public values- and use-based socio-spatial data into the planning process to ensure legal compliance.

The NFMA requires forest plans be assessed and/or revised on a ten-year schedule. A large percentage of the existing forest plans have undergone plan revisions under the older 1982 rule. Eight forests were selected as "early adopters" for forest plan revision under the new 2012 rule and numerous other forests also proceeded with plan revisions under the new rule. This provides a means for a comparative analysis between forests that submitted plans under the 1982 rule and a few that are conducting the process under the 2012 rule.

4. In a 2015 review of PPGIS projects in natural resource management, 13 human ecology mapping projects were identified that took place on U.S. national forests. Despite making the socio-spatial data freely available to forest planners, only one of the academic articles reporting these projects note significant use of the data in actual forest planning (Biedenweg et al., 2014). However, the fact that these studies have been done on national forests provides a unique opportunity to explore more deeply how the kind of socio-spatial data these projects generated might have informed forest plan revision in both direct and indirect ways.

\subsection{U.S. Forest Service Organizational Structure}

Three administrative units of the USFS are considered in this study - the Regional Offices, National Forests and Research Stations. Each of these units incorporate social science in one form or another in their mission and practice, but have different functions 
within the agency. Including all three levels allows for exploration of similarities and differences in how staff in each level think about and incorporate socio-spatial data into their operations and decisions. To provide background information and context for discussions in the chapters that report the findings (Chapter 7) and offer recommendations (Chapter 8), following is a brief description of the USFS National Forest System and the forest management plan revision process.

The National Forest System (NFS). The NFS is a program unit within the USFS that is responsible for managing public forests and grasslands assigned under its jurisdiction. The NFS has several administrative levels that provide coordination of services and programs from a national to regional to local level. Figure 4 provides a graphic of the hierarchy between these levels.

Figure 4: U.S. Forest Service, National Forest System administrative levels.

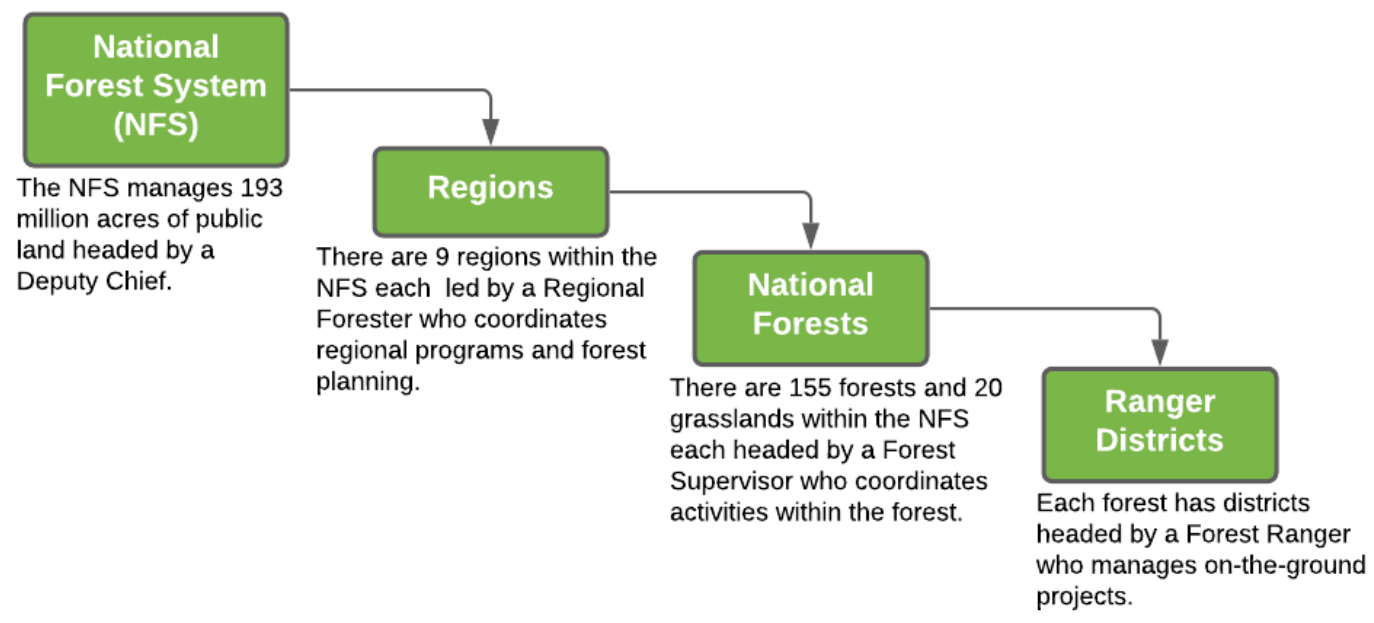

Source: Adapted from USFS Agency Organization at Agency Organization | US Forest Service (usda.gov).

Under the National Forest System, each of the nine regional offices is headed by a regional forester. The regional forester has broad responsibility and decision-making authority for supervising activities among the various forests within the region, allocating 
budgets for the forests, providing overall leadership for regional natural resource and social programs, and coordinating land-use planning. Program managers and scientists at the regional offices provide guidance and expertise for forest-level programs and projects, management plan development and forest monitoring activities.

Each region has numerous national forests or grasslands within its territory ranging in size from 17 million acres (Tongass, AK) to 11,000 acres (Tuskegee, AL). Regional office and forest-level staff work closely together, sharing expertise and resources as necessary to implement management objectives within the forests. Each national forest is headed by a forest supervisor that serves as a liaison with the regional office and coordinates staff and activities within the forest. The forest supervisor is also responsible for completion of the comprehensive forest management plan and, under the new 2012 planning rule, making the final decision on which plan option will be adopted. Forest supervisors also develop and maintain collaborative relationships with forest stakeholder groups, local governments and members of the public.

Forests are then divided into districts each headed by a forest ranger. Each district may have 10 to 100 staff members depending on size of the district and the complexity of management priorities. The forest ranger manages staff and on-the-ground projects within the district, such as trail construction, habitat restoration, and public outreach.

Forest plan revision occurs within this complex organizational hierarchy and involves intricate coordination of information and resources both top-down (e.g. budget and staffing allocations) and bottom-up (e.g. forest assessments; public input). Coordination side-to-side also occurs as scientists at the USFS research stations or at academic institutions may be asked to assist in producing a science synthesis or 
additional scientific analyses on topics of concern or interest. The next section provides a description of the forest plan revision process.

\subsection{Comprehensive Forest Management Plan Revision Process}

The National Forest Management Act of 1976 (NFMA) requires that the Secretary of Agriculture evaluate forest lands, develop a management program based on multiple-use, sustained yield principles and implement a resource management plan for each forest unit within the NFS. The forest plan development process is a lengthy, intense and resource heavy effort designed to assess the state and health of the entire forest and prepare a management plan, with comprehensive stakeholder input, that meets diverse social, environmental, economic, recreational and cultural objectives. The National Environmental Protection Act of 1969 (NEPA) also requires that any forest management plan follows a strict process and includes an environmental impact statement subject to a public comment and objection/appeal period. The administrative rules on how forest management plans are to be constructed are outlined in the Forest Planning Rule. The Planning Rule provides detailed instructions on what data to collect, who to involve and how to compile into a comprehensive plan (see page 39 for a more detailed description of the Planning Rule). The Forest Service Directive System consists of the Forest Service Manual and Handbooks, which "codify the agency's policy, practice, and procedure. The system serves as the primary basis for the internal management and control of all programs and the primary source of administrative direction to Forest Service employees" (Forest Service Directives: Forest Service Directives (fs.fed.us)).

Figure 5 below shows a simplified graphic of steps in forest plan revision, a protracted, highly structured process governed by stipulations in both NEPA and the 
Forest Planning Rule. Each stage entails numerous activities with distinct outcomes or products. Despite the complexity of this process, and the strict rules that must be followed at each stage, the USFS often faces intense public and political scrutiny to be efficient, cost-effective and scientifically sound, while also ensuring comprehensive outreach and involvement of all stakeholders (Koontz, T. M., 2002). Preparation of each forest plan can take $4-5$ years or more to complete.

Figure 5: The U.S. Forest Service forest management plan revision process.

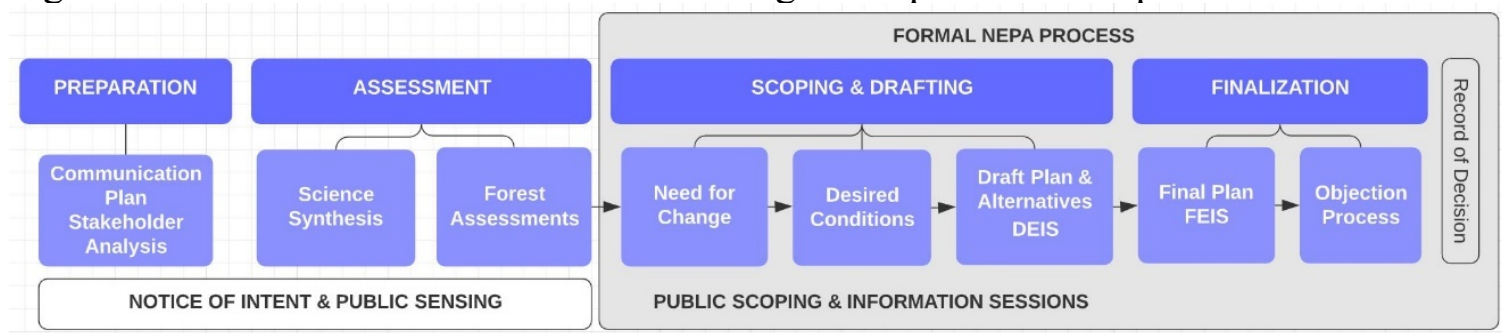

Source: USFS Planning Rule 101 (https://www.fs.usda.gov/main/planningrule/101).

The Plan Revision Process. The process begins with a public Notice of Intent to revise the existing forest management plan, followed by preparation of a comprehensive forest assessment. Existing data is collected to determine the current state of the forest to provide information for a formal public statement indicating a Need for Change (justification that a revision of the current management plan is required) and a Desired Conditions document (which describes new management objectives). While public input during the assessment phase is not legally required (the formal NEPA process begins with the Need for Change notice), it often occurs during the assessment phase to begin to engage the public and gather information to inform the forest assessment. These activities are often referred to as public "sensing" and include information sessions (e.g. what is a forest plan and how does the revision process work?), workshops on various forest management challenges that indicate a need for plan revision, and different kinds 
of surveys to gauge public values, interests and how they use forest resources in order to inform the forest assessments and desired conditions statement.

Preparation of a draft plan commences once a desired conditions statement is finalized. This is often referred to as the "black box" by study participants. This is the point where USFS specialists retreat to their corners and construct a draft plan that contains numerous alternatives and an accompanying environment impact statement. These draft plans can be hundreds of pages and take a year or more to complete. Challenges occur in keeping the public informed and engaged during the lengthy time frame between preparation and publication of the draft plan. Public input is again solicited once the draft plan has been released to gather public reactions to the plan's proposals. At this point, more targeted public participation strategies are often employed that focus on public reactions to various management components, such as proposed management boundary units, wilderness designations, recreational areas, etc. This assists the forest supervisor in selecting one of the alternatives that will become the final plan. A formal NEPA public comment and appeal process occurs at the point the final plan is submitted. The agency is required to address all comments and provide a justification for a decision, that a commenter may or may not appeal. This is the point where formal organizations and interest groups (e.g. the Sierra Club or Timber Associations) often exert the most influence (Koontz, 2002). These groups have the resources and legal acumen to take advantage of the formal comment and appeal process to put forth their agendas. Public input into the decision process is a deliberate aspect of NEPA, to make sure a diverse set of interests are entered into the decision calculus. However, these formal legal processes have subtle (or perhaps not so subtle) influence on how the plan 
revision process is conducted. The extent to which the plan revision process can meet this NEPA legal test - from compilation of data that informs assessments to construction of the plan components to the decision matrix used to select the final plan - is a significant consideration during all phases of the process that can potentially impact decisions on what data is collected and how it is incorporated into the documents.

Forest Planning Documents. The forest plan revision process produces numerous formal documents including: (1) forest assessments that synthesize existing research and data ${ }^{1} ;(2)$ a draft plan with alternative management proposals and accompanying draft environmental impact statement (DEIS); and (3) the final plan and final environmental impact statement (FEIS). In addition, a forest may publish reports on the public engagement strategy and a synopsis of comments and input from interest groups and the public at large collected throughout the process.

Forest assessments contain different sections for various ecological and human considerations, such as wilderness, timber, recreation, and cultural services. Their purpose is to synthesize existing Best Available Scientific Information (BASI) on current forest conditions and management issues. There is no standard definition of what constitutes best available science. BASI in the context of forest management generally includes data collected via accepted scientific methodologies within the natural, biophysical and social sciences, which most often take a positivistic approach, emphasizing quantitative analysis or sophisticated modelling with what would be

\footnotetext{
${ }^{1}$ The Forest Planning Rule specifically states that synthesis of research should be comprised of existing relevant data although gathering new data to fill what is deemed a critical gap is not explicitly disallowed and is at the discretion of the Forest Supervisor (National Forest System Land Management Planning, 36 CFR §219.6(1)).
} 
considered statistically valid conclusions (Charnley et al., 2017). This is problematic as it can exclude consideration or incorporation of equally valid qualitative data that compiles and analyzes social, cultural and psychological aspects of public relationships with forests, but uses interpretive analytical strategies.

Forest assessments are expected to be compiled using BASI. Thus, the 'human' areas of interest often rely on demographic and economic analyses using government databases such as the U.S. Census, for demographic information, and Department of Labor databases, for economic assessments. Other data collection and modeling tools are used to capture human-environment interaction such as the Recreation Opportunity Spectrum (ROS), the National Visitor Use Monitoring or NVUM (done every five years), and the Scenery Management System (SMS), that quantifies the visual aspects within the forest. It is important to note that the ROS and the SMS are analytical models utilized by skilled practitioners.

The draft and final plans are documents that describe the goals of the forest management plan. The draft plan puts forth several possible options (the alternatives) for public review and comment; the final plan is the choice of one of the alternatives, which then goes through a formal NEPA public comment and appeal period. The draft and final plans are typically not useful for identifying specific socio-spatial data that might have been integrated, explicitly or implicitly. They typically reference the forest assessments as the source documents for any statements made in the plan alternatives. The DEIS and FEIS (the accompanying draft and final environmental impact statements) are somewhat more explicit and describe the public involvement process as well, but generally do not provide details on any socio-spatial data collected during public meetings. 
While a formal document analysis was not conducted for this study, available planning documents for the sample forests included in this case study were reviewed to determine the extent to which they identify use of socio-spatial data collection tools or incorporation of socio-spatial data in the assessments and draft plan. This document review for each sample forest is described in more detail in the forest briefs that are attached as Appendices D, E, F, and G.

The Interdisciplinary (ID) Team. To manage the process and compile the numerous analyses required to produce a management plan, an interdisciplinary team (ID team) is put together that coordinates the effort and keeps the process moving forward. The composition of the ID teams vary from forest to forest. Some teams are made up of dedicated staff (100\% time throughout the process) while others are composed of staff with only limited hours allocated. Many teams rely on outside temporary contractors to provide subject-specific analyses (e.g. economic modeling) or facilitation of the public participatory process. Additional assistance is provided by regional office scientists and program staff. However, it should be noted that there are few social scientists assigned to regional offices, even less at the forest level. Staff capacity issues are a significant challenge. The Forest Planning Rule lists 15 required assessments for inclusion in the forest plan, five of which speak to social, cultural or economic conditions: (1) existing social, cultural and economic conditions; (2) tangible benefits people obtain from the forest (ecosystem services); (3) multiple uses and their contribution to economies; (4) recreation settings and scenic character; and (5) inventory of cultural and historic resources (National Forest System Land Management Planning, 36 CFR §219.6(b)). When a social scientist's time is consumed with producing the BASI-compliant 
comprehensive analyses that are required for the forest assessments, there is often little time left to attend to what might be deemed valuable, but not required, such as incorporating an assessment of public values toward a forest using socio-spatial data.

\subsection{U.S. Forest Service Research Stations}

The discussion thus far has focused on the National Forest System. A different functional unit is also considered in this study, the research and development (R\&D) arm of the USFS. The R\&D unit is administratively separate from the NFS. This program unit has its own mission, objectives and standards of practice focused on producing cutting edge research to inform and improve forest management policy and practice. Scientists at the research stations carry out basic and applied research to study biological, physical and social phenomena related to forests and rangelands. Scientists also partner closely with universities and the international research community to address compelling and consequential issues facing natural resource management.

As a program unit, the R\&D arm of the USFS is quite small. Approximately 500 scientists are employed at five research stations and several experimental forests, representing slightly over $1 \%$ of the total workforce (USFS Research Stations: https://www.fs.usda.gov/research/). The number of researchers working in the social sciences is even smaller. Social scientists at the research stations concentrate on scientific inquiry and are not typically directly involved in forest planning or management. However, due to the scarcity of social scientists within the agency as a whole, social scientists at research stations are often called upon to assist in compiling the science synthesis that informs forest plan revision or writing technical briefs and white papers distilling current research on particularly urgent management issues. However, 
promotional incentives for scientists at the research stations focus on published work in peer reviewed journals. There is little advantage in producing technical reports or literature reviews. The implications will be discussed in subsequent chapters as it relates to supporting the collection of socio-spatial data during the plan revision process.

\subsection{Paperwork Reduction Act and Office of Management and Budget Oversight}

The Paperwork Reduction Act of 1980 (44 U.S.C. $\S 35501-3521$ ) was enacted to relieve the public of the mounting information collection and reporting requirements of the federal government. It also assigned information management activities and regulatory oversight to the Office of Management and Budget (OMB). OMB oversight functions related to the collection of information by federal agencies include: (1) reviewing and approving information collection requests proposed by agencies; (2) determining whether the collection of information by an agency is necessary for the proper performance of its functions; (3) ensuring that all procedural requirements for collecting information are fulfilled; and (4) setting goals for reduction of the burdens of Federal information collection requests (44 U.S.C. 3504(c)).

The OMB is given expansive authority to make determinations on what agencies must submit around solicitation of public information as well as in making judgements around whether agency proposals meet a plethora of criteria. However, as Morrison (1986) argues, the intent of the PRA (and OMB oversight) to reduce the burden on the public has resulted in overburdening federal agencies. As Morrison states, “...the vast amount of additional [Federal] resources spent in...obtaining the necessary OMB clearance to undertake the studies needed to decide whether to begin work on a problem in earnest, are all burdens on the federal treasury [the cost and time of producing these 
proposals], yet there is no indication that these costs have been balanced against the benefits to be derived from this complex labyrinth of OMB overlay" (Morrison, 1986, p. 1066). ${ }^{2}$ An additional issue that Morrison points out is that Federal agencies spend vast amounts of resources preparing detailed proposals for OMB approval that must explain scientific processes and outcome objectives to OMB desk officers who have the authority to decide on the proposal's merits without the requisite scientific knowledge about the subject matter. This often results in OMB rejection of the proposal or can extend the approval process over months and even years as agencies struggle to make their case.

The need for OMB clearance for public data collection is an external contingency that significantly affects USFS social science researchers, as surveys, interviews, focus groups and other tools that involve public input are standard social science data collection methods. It is not surprising that social scientists working on preparation of forest assessments rely heavily on existing data mined from other U.S. government databases, such as the U.S. Census. Proposing the collection of new socio-cultural (or socio-spatial) data would require entering the OMB "labyrinth" and perhaps waiting a year or more for approval. That is not possible given the staff capacity issues and tight time frames of a forest plan revision process.

Summary. What should be evident from this discussion about the NFS hierarchy, the planning process and the regulatory environment imposed by the NEPA, NFMA, PRA and OMB is complexity, formality and interconnectedness. The planning process, in particular, is highly formalized, ordered and deeply intertwined with NEPA

\footnotetext{
${ }^{2}$ Though this article dates back to 1986, shortly after enactment of the PRA, amendments to the legislation since have not addressed the concerns Morrison expresses in this quote (see Relyea, 2000, for an excellent legislative review of the Paperwork Reduction Act).
} 
and Planning Rule legal and procedural requirements in timing and staging (what must happen and when). What kind of data to collect (existing BASI) is influenced by the Forest Planning Rule and the potential OMB oversight with regard to collecting "new" data. The process, as currently conceived seems highly prescribed, with potential impacts on the ability to advocate for innovative ideas or inclusion of new types of data collected from the public. Coordination of all these parts to produce an output is a systems-based challenge that will be explored further using study participant reflections and responses. The next two chapters turn to describing the methods and strategies adopted to collect and analyze study participant input. 


\section{DATA COLLECTION STRATEGY}

I selected the semi-structured interview as the primary data collection tool for this study because, on the one hand, I needed the interviews to cover the broad categorical areas in the initial conceptual framework. On the other hand, I needed relevance of and linkages between the possible factors listed within those categories to be identified by participants in a more organic fashion. What did they think was important within those broad categories? How do participants place these factors within the larger systemic context? Semi-structured interviews serve this purpose by incorporating both open-ended and more intentional or structured prompting questions, eliciting data grounded in the experience and insights of the participant as well as data guided by the framework that informs the research topic.

\subsection{Semi-Structured Interviews}

I developed interview protocols, informed by Galletta (2013) and reviewed by my technical advisors, which guided the interviews with USFS practitioners, scientists and scholars. Using protocols with prompting questions mitigates the potential to veer into unrelated, but perhaps very interesting, topics as well as inhibiting suggestive comments from the interviewer that might solicit canned or expected answers (Galletta, 2013). The interview protocols are attached as Appendix C.

There are advantages and limitations associated with use of semi-structured interviewing. The structured part of the process will limit the free-flow of responses by intentionally focusing prompting questions on specific categories of interest. However, parroting and probing techniques can uncover nuances within these areas. In addition, the open-ended aspect of this interviewing technique often results in large segments of 
text that contain numerous references, links, insights and opinions that can be difficult to parse or synthesize. Several qualitative analytical tools are available to aid in this process, such as the analytic memo and the cut-and-sort technique for organizing large amounts of textual data. These methods have been applied in this study and will be explained further in Chapter 6. As is the case with all research, researcher bias must be identified and considered. In the act of conducting the interview, the researcher becomes an actual participant with the potential to influence the respondent's comments.

Researcher bias is discussed in the Reciprocity and Reflexivity section below. Table 2 summarizes the advantages, limitations and mitigation strategies of the semi-structured interview.

Table 2: Advantages, limitations and mitigation strategies in semi-structured interviewing.

\begin{tabular}{|l|l|l|}
\hline \multicolumn{1}{|c|}{ Advantages } & \multicolumn{1}{|c|}{ Limitations } & \multicolumn{1}{c|}{ Mitigation Strategies } \\
\hline $\begin{array}{l}\text { Allows engagement with } \\
\text { participants in an intentional } \\
\text { manner that addresses research } \\
\text { objectives. }\end{array}$ & $\begin{array}{l}\text { Often multi-dimensional streams } \\
\text { of data, making it difficult to } \\
\text { segment data and/or find } \\
\text { connections. }\end{array}$ & $\begin{array}{l}\text { Field notes add contextual } \\
\text { information. } \\
\text { Analysis tools, such as the } \\
\text { analytic memo and cut-and-sort } \\
\text { techniques, provide a structured } \\
\text { way to group and interpret text } \\
\text { segments. }\end{array}$ \\
\hline $\begin{array}{l}\text { Allows for increased } \\
\text { understanding by probing } \\
\text { participant responses for clarity, } \\
\text { meaning making and critical } \\
\text { reflection (as contrasted with use } \\
\text { of surveys with targeted } \\
\text { questions). }\end{array}$ & $\begin{array}{l}\text { Questioning is subject to } \\
\text { researcher influence or bias, } \\
\text { particularly in probing for details } \\
\text { researcher, thus missing } \\
\text { opportunities to explore other } \\
\text { potential topics. }\end{array}$ & $\begin{array}{l}\text { Consistent use of interview } \\
\text { protocols. } \\
\text { Focus on parroting and probing } \\
\text { techniques while interviewing. } \\
\text { Self-reflection on potential } \\
\text { researcher bias through use of } \\
\text { field notes and the analytic } \\
\text { memo. }\end{array}$ \\
\hline
\end{tabular}

Source: Adapted from Galetta, 2013.

When conducting interviews, housekeeping matters were addressed first, including giving the participant a short synopsis of the research, answering any questions, and securing either a verbal (phone interviews) or signed consent form (in-person 
interviews). This was followed by asking a few questions to gather basic descriptors from participants (education, career trajectory, length of agency service), which allows for parsing data into sub-groups of participants for data analysis. The remaining interview inquiries were designed to elicit information about the participants' experience, opinions, feelings, knowledge about, and input into the forest planning process or related activities. Specific questions were included to probe knowledge about the use of social science and/or collection of socio-spatial data in support of the planning process. The protocols served as guides only. Participants were encouraged to talk about what they felt was most important after being given a general prompting question. Information about the study that was provided to the participant and the interview protocols can be found in Appendix A (Study Prospectus), Appendix B (Consent Form) and Appendix C (Interview Protocols).

Field Notes. After each interview I prepared supplementary notes, often called field notes. These notes add observations not included in the transcript and capture aspects of the interview that may be lost in segmentation of the data, such as additional context or any strong emotional reactions that the participant may have exhibited around a particular question, but not clearly evident in the interview transcript. In the notes, I also summarized the major themes the participant identified for future reference. I made note of where my own bias may have entered or influenced the conversation (for example, areas in which I felt that I may have unduly inserted my own opinion) in order to take this into account during the coding of interview transcripts. These notes were continually updated during the iterative reading and re-reading of the interview transcripts during the thematic analysis phase. 


\section{Reciprocity and Reflexivity in Semi-structured Interviewing. A necessary}

area of consideration in qualitative research is the role of the researcher as a participant in the study itself. During an interview, the researcher personally engages with participants in both a reciprocal and reflexive manner. Galletta (2013) notes that this is particularly true when the semi-structured interview is used as a data-collection method. While the intent is to allow the participant to steer the dialogue, it is, nonetheless, a complementary back-and-forth exchange. In addition, the researcher is constantly making judgements on what to probe more, where to take the next question, and may reposition, rephrase or add questions according to the particular flow of the interview. While this is accepted practice in interviewing in order to take advantage of unexpected insights and information the participant may offer, a great deal of reflexive thought is also needed on the researcher's part to avoid the trap of 'seeking and finding the answers you expect.' Numerous strategies and techniques are used in this study to mitigate these challenges including:

- Adhering to interview protocols as a guide to maintain focus on a broad set of questions;

- Constructing field notes to identify possible bias or influence for later reflection (where the interview might have veered from the protocols);

- Focusing on parroting and probing interviewing techniques, rather than engaging in a free-for-all discussion;

- Using analytic memos to synthesize numerous transcripts (these memos ask a standard set of reflective questions of each interview focusing on the research objectives and help to eliminate narrative that may be off topic);

- Adding text tags to transcripts to assess the impact of possible researcher bias or influence on a particular text segment; and

- Employing numerous data analysis tools that aggregate coded text segments using different analytical objectives (this allows for triangulation of results to improve validity). 


\subsection{Participant Selection Process}

A purposive sampling technique was used in this study in order to recruit a broad spectrum of participants from a pool of scientists and practitioners within the USFS's three administrative levels (research stations, regional offices and national forests). An email was sent to selected persons providing information about the study (see Appendix A: Study Prospectus) and inviting them to participate, with several follow-up emails and/or a phone call if no response. To encourage participants to speak freely, the consent agreement guaranteed confidentiality, though not anonymity (see Appendix B: Study Consent Form). Excerpts from interview transcripts will be used for evidentiary purposes. No names of participants will be reported.

Leading scholars who have published extensively on the human dimensions of public land management, place-based forest planning, and tools for collecting sociospatial data were also contacted and invited to participate. Three scholar interviews were completed. Transcripts from these interviews are not included in the aggregate analysis. These interviews were used to derive insights into the theoretical and practical implications of the study findings.

USFS Regional Offices and Research Stations. Outreach to the USFS's nine regional offices and five research stations focused on forest service employees with titles such as social scientist or those involving the social sciences (e.g. geographer, anthropologist, economist). The list of potential participants was not extensive as the number of social scientists working at regional offices or the research stations is quite small in comparison to the number of physical and biological scientists at the agency. Attempts were made to contact numerous social scientists at each regional office and 
research station drawn from staff lists on the USFS website. Three social scientists from the Pacific Northwest research station and two from the Northern research station were interviewed. Five social scientists from the regional offices were interviewed representing Region 2 (Rocky Mountain), Region 5 (California), Region 6 (Pacific Northwest), and Region 8 (Southern).

National Forest Planning Team Members (ID Team). The USFS manages 174 forests and grasslands and nine national monuments in 43 states, Puerto Rico and Guam. The intent was to send an invitation to participate in the study to forest planners and other practitioners from two forests preparing forest management plans under the older 1982 forest planning rule and two using the new 2012 rule. An iterative selection process was employed to scale down the list of 174 forests to potential sample forests. The process consisted of an elimination round to reduce the list to forests actively involved in forest plan revision followed by ranking the semi-finalist forests by the planning stage and the potential availability of socio-spatial data. The forest selection process is described below.

First Round of Forest Elimination. A web search of all national forests was conducted in early 2015 to determine the status of their forest plan revision process and the availability of forest assessments, draft or final plans and environmental impact statements. The goal was to compile a list of forests where staff were actively engaged in forest plan revision. The criteria eliminating forests from the first round included:

1. Forests that were not doing any plan revision.

2. Forests that completed plan revision over 1 year prior to the search and had submitted a Record of Decision (under the assumption that the planning process was a distant memory). 
3. Forests where the website indicated that forest plan revision was in process, but had no information, schedule or reports available.

4. Forests undergoing plan revision under the 2012 rule, but were in the very early stage with perhaps a Notice of Intent, but no substantive information on public engagement or forest assessments were available.

From the full list of national forests, 12 were potential candidates representing forests in seven of the nine regions. There were no forests in the intermountain region (NV, UT, parts of southern ID and western WY) or eastern region (generally the northeastern and midwestern states) undergoing plan revision at the time of the search.

Ranking the Sample Forests for Final Selection. The 12 forests that were well into the revision process or who recently completed plan revisions were separated into: (1) forests that were revising or had recently submitted forest plan revisions under the 1982 rule, and (2) forests that were currently in the forest plan revision process under the new 2012 rule. Since the 2012 planning rule contains revised language emphasizing public collaboration and socio-cultural considerations, having a forest sample representing both planning rules allows for comparative analysis. ${ }^{3}$ The semi-finalist forests were ranked based on two criteria:

Stage of Planning (0-4): The highest ranking is given to forests at stages in the planning process where the greatest level of public involvement and input is typically generated and socio-spatial data is likely to be collected and/or considered. The lowest rankings reflect stages in which minimal public input takes place. The stages identified below are listed in a relatively linear process as they occur; each stage may require a year or more to complete.

\footnotetext{
${ }^{3}$ Forests already well into the process of preparing a revised forest management plan under the 1982 rule, at the time the 2012 rule was formally registered, were allowed to finish the process under the old rule.
} 
Stage 1 (3 pts.): Notice of Intent and compilation of forest assessments, which may include a science synthesis and/or stakeholder analysis; this stage typically includes "public sensing" activities and information sessions.

Stage 2 (4 pts.): Development of Need for Change \& Desired Conditions statement; preparation \& release of draft plan with alternatives; formal NEPA public input through public "scoping" to gather stakeholder feedback and assist in making a final decision; this stage typically includes the most public input.

Stage 3 (2 pts.): USFS decision-maker chooses the final plan from the draft alternatives; after release of the final plan, public comments are solicited, reviewed and reconciled; the final plan may or may not be revised based on this input; public input at this stage is limited to formal commenting per NEPA requirements.

Stage 4 (1 pt.): NEPA formal objection/appeal process \& Record of Decision. Stalled ( 0 pt.): Planning process far along (past assessments) but seems to be stalled; no draft plan or other information is available.

Presence or Absence of Previously Collected Socio-spatial Data. Based on my 2015 review of HEM literature, researchers conducted projects collecting socio-spatial data (either through human ecology mapping or public values-based surveys) in eight of the national forests that made the candidate list for this study. Whether regional office social scientists or forest planners were aware of this data or used it in any way (directly or indirectly) is a primary research question. Forests that potentially had socio-spatial data at their disposal were given a ranking of 1 ; those without received a 0 .

Consideration was also given to selecting forests that represent different geographic regions. Eastern and western forests have different ecological, social and 
economic challenges as well as different management priorities and public engagement

histories. Forests in the semi-finalist list represent seven of the nine regions. Table 3

shows 12 forests (or multiple forests when combined for plan revision purposes) that

were considered in the final selection round with their total score.

Table 3: National forests conducting forest plan revisions (as of Nov. 2015) and associated rankings based on the study's participant selection criteria.

\begin{tabular}{|c|c|c|c|c|c|}
\hline Forest Name* & $\begin{array}{c}\text { Plan Rule } \\
\text { Year }\end{array}$ & Region & $\begin{array}{l}\text { Planning } \\
\text { Stage** }\end{array}$ & $\begin{array}{c}\text { Socio-Spatial } \\
\text { Data } * * *\end{array}$ & Score \\
\hline FLATHEAD & 2012 & 1-MT & 4 & 1 & 5 \\
\hline Rio Grande & 2012 & $2-\mathrm{CO}$ & 3 & 0 & 3 \\
\hline Carson & 2012 & 3-NM & 3 & 0 & 3 \\
\hline Cibola & 2012 & 3-NM & 3 & 1 & 4 \\
\hline Inyo, Sierra, Sequoia & 2012 & $5-\mathrm{CA}$ & 4 & 1 & 5 \\
\hline Pisgah \& Nantahala & 2012 & $8-\mathrm{NC}$ & 3 & 0 & 3 \\
\hline FRANCIS MARION & 2012 & 8-SC & 4 & 1 & 5 \\
\hline Chugach & 2012 & $10-\mathrm{AK}$ & 4 & 1 & 5 \\
\hline Kootenai, ID Panhandle & 1982 & $1-\mathrm{MT} / \mathrm{ID}$ & 3 & 1 & 4 \\
\hline COCONINO & 1982 & 3-AZ & 4 & 1 & 5 \\
\hline Coronado & 1982 & $3-A Z$ & 0 & 1 & 1 \\
\hline COLVILLE & 1982 & 6-ID/WA & 4 & $\mathbf{0}$ & 4 \\
\hline
\end{tabular}

*No national forests from Region \#4 (Intermountain) or Region \#9 (Eastern) were in the process of forest plan revisions and are not reflected in this list.

$* * 3$ pt. $=$ Stage 1 , Notice of Intent and development of forest assessments.

4 pt. $=$ Stage 2, Need for Change \& Desired Conditions statement; preparation \& release of draft plan.

2 pt. $=$ Stage 3 , Preparation \& release of the Final Plan.

1 pt. $=$ Stage 4 , NEPA objection process \& Record of Decision.

$0 \mathrm{pt} .=$ Planning process seems to be stalled and no substantive reports or other information is available.

***Forests where a human ecology mapping project had taken place received a score of 1; if not, the forest received a 0 .

The Flathead, Inyo/Sierra/Sequoia, Francis Marion and Chugach National Forests received the highest ranking for forests revising under the 2012 planning rule. The Coconino, Colville and Kootenai/Idaho Panhandle National Forests received the highest 
ranking for those revising under the 1982 rule. Study information and an invitation to participate were sent to the forest supervisors for all seven candidates, as a precursor to approaching forest staff. The goal was to interview three staff members at each sample forest that were involved in the plan revision process, ideally members of the ID planning team.

Selection of the four final forests was subject to some logistical constraints. The Inyo/Sierra/Sequoia, Chugach and Kootenai/ID Panhandle were eliminated because forest staff did not respond to my invitation or indicated unwillingness to participate (e.g. too busy). In these cases, the next forest in the ranking was selected. The four finalists included in this study are the Flathead, Francis Marion, Coconino and Colville National Forests and are noted in bold in the table above. From these forests, ten forest staff were interviewed. Interviews from an additional three forest-level staff with particular expertise and experience (e.g. social science in planning, environmental law) are also included in the aggregate analysis. In total, 13 interviews were conducted with forestlevel staff.

\subsection{Sample Forest Briefs}

The following section contains summaries for each sample forest. These summaries provide important information and context about the selected forests, their unique management challenges, their planning process and timelines, and information about socio-spatial data collected during this process. Further detail, including selected participant comments, can be found in Appendix D (Flathead Forest Brief), Appendix E (Colville Forest Brief), Appendix F (Coconino Forest Brief), and Appendix G (Francis Marion Forest Brief). 
Flathead National Forest. The Flathead National Forest, located in northwest Montana, is one of the largest in the National Forest System. It is part of a conglomeration of forests in northern Idaho and northwest and western Montana that have historically been the most productive timber producing areas in the country. Management challenges in the Flathead reflect the immense diversity of its resources and users. The forest must (1) address the conservation needs of numerous protected and endangered species; (2) maintain the quality of and access to a wide range of forest resources for local communities, such as foraging, hunting and fishing; (3) meet timber production quotas; (4) provide a wide variety of year-round motorized and non-motorized recreation in developed and backcountry settings; and (5) manage the uncertainties of climate change and its effects on the environment, including an increasing threat from wildfire. In addition, development of the forest's management plan requires the inclusion and input of numerous entities including tribes, federal and state agencies, local communities, private landowners, and a dizzying number of local, regional and national special interest groups. Understandably, conflict in values and user preferences between various stakeholder groups constitute the primary planning challenge.

The Flathead completed its plan revision process in four years using the 2012 planning rule. Its Record of Decision was submitted in late 2018. Interviews from Flathead participants pointed to two important operational decisions that helped staff move forward quickly - a full-time ID team and an external facilitator to manage what was expected to be a rather contentious public engagement process. According to participants, having a dedicated ID team allowed staff to invest the time and energy needed for this massive undertaking (the final plan is over 400 pages, not including 
appendices). ID team members also were not burdened with developing and implementing the extensive public participation strategy needed to engage numerous forest stakeholders. The forest contracted with an outside facilitator, specializing in conflict management, to implement the strategy. The primary goal was to bring stakeholders together, educate them about forest management priorities and challenges, and try to come to some reconciliation between numerous competing interests. Study participants from the Flathead indicated the task was immensely challenging (and often frustrating) and required a highly structured plan of action to keep public interactions directed and productive.

Various participatory mapping strategies were used purposefully and extensively to focus the public on key decision areas. Socio-spatial data was gathered in a multitude of formats - sensing surveys, hardcopy maps used at public workshops, and online mapping applications - informing the planning process both explicitly and implicitly. Maps were used to engage the public in discussions around water and species conservation needs, roadless areas, recreation zoning, proposed wilderness areas, and boundaries of management areas, which were revisited and revised as a direct result of public input on the draft plan alternatives.

[Information on the Flathead's forest plan revision process can be found at the Flathead Forest Plan Revision webpage: Flathead National Forest - Planning (usda.gov).]

Coconino National Forest. The Coconino National Forest is located in northcentral Arizona and is bordered by four other national forests. Population growth in the surrounding urban areas and increasing tourism shifted management priorities from traditional commodity production (e.g. timber, grazing and mineral extraction) to a much stronger emphasis on day-use recreation. Contemporary challenges include increased 
demand for developed and more diverse year-round recreational opportunities that often resulted in use conflicts, such as between snowmobilers and cross-country skiers.

The Coconino experienced fits and starts in its plan revision process. The forest started its plan revision in 2006, first using a 2005 planning rule then a 2008 rule, both of which were rescinded by the courts. Planning resumed in 2010 (reverting back to the 1982 planning rule) with a sense of urgency, exacerbated by lack of consistency in leadership and within the planning team due to high staff turnover during the extended lag time. The Coconino submitted their record of decision in early 2018.

Participant comments for the Coconino indicate that the primary goal, at the time of the interviews, was to "get the job done quickly," as there was increasing pressure to finish under the old rule and move on. ${ }^{4}$ At the time of the interviews, the planning team had submitted a draft plan for public review. They admitted that when planning resumed in 2010 , they had little capacity or resources to update data already compiled or collect new data, socio-spatial or otherwise. Of note, a published article indicates that a HEM project collecting socio-spatial data on the Coconino was completed in 2007 (Brown \& Reed, 2009), but those interviewed had little memory of the project or the data produced. They used the values and attitude surveys that were completed in 2006 as part of the public sensing process, as outdated as they might be, and a constituent analysis that consolidated public perceptions of attractiveness and identified special places that were incorporated into the scenery report. Though Coconino participants struggled with

\footnotetext{
${ }^{4}$ One Coconino study participant noted that the forest was under a strict timeline to finish under the 1982 planning rule. If they did not meet that timeline, the forest would be required to start over using the new 2012 rule directives.
} 
discussing socio-spatial data in the context of their plan revision effort, one participant did try to articulate the value of the data in a broad sense:

Initially, when we kicked off plan revision efforts, yes, we tried the approach of both kinds of map-based comments and then topic-based comments. And I think that was a great way to get people involved because while we make these artificial distinctions that this is the [forest] and here are the districts and all these boundaries and stuff, that's not how people relate to the land. I think that was pretty successful, and then there was additional effort made for input on wilderness, wild and scenic rivers, and then other special area interest designations and that sort of thing.

[Information on the Coconino's forest plan revision process can be found at the Coconino Forest Plan Revision webpage: Coconino National Forest - Planning (usda.gov)].

Francis Marion National Forest. Located on the east coast of South Carolina and surrounded by rapidly growing urban areas, forest planning priorities on the Francis Marion concentrate heavily on providing diverse recreational opportunities for the surrounding urban residents which need to be balanced with protection of fragile coastal ecosystems under constant threat from hurricanes. Increasing recreation pressure, and its potential for environmental degradation, was identified as the most pressing planning challenge.

Like the Flathead, the Francis Marion also commenced with plan revision using the 2012 planning rule and completed the process in about four years, beginning in 2013 and submitting a Record of Decision in early 2017. A significant asset that Francis Marion participants noted led to a smooth plan revision process is the long tenure of many forest staff. This allowed staff to develop what they considered strong and positive relationships with the surrounding communities and the many "friends of" groups active in the forest. Members on the ID team leveraged these relationships and committed early to providing a comprehensive and multi-tiered public engagement process which they 
called "community conversations." They felt this investment in public engagement was critical in gathering data on public preferences and values to inform forest plan components as well as creating a shared vision of how to manage the forest's resources. Of note, these community conversations continued throughout the entire revision process, including several interim releases of the draft plan for public review. This is not a common practice as planners feel this would result in never-ending tweaking of the draft plan. However, Francis Marion planning team members felt this was instrumental in public acceptance of the final plan and did not seem to affect their timeline.

Numerous mapping activities supported this effort and occurred at all stages of the planning process. The community conversations model consisted of workshops (and later an online application) that provided canvases and maps with prompting questions. Both spatial and non-spatial data were compiled about (1) the benefits the public receives from the forest; (2) what is unique and special about the forest; (3) the public's favorite places to recreate and commune with nature; and (4) what might make a visit to the forest better. In addition to informing plan components, the socio-spatial data collected were instrumental in creating resource integration zones (that were integrated with the more traditional management priority zones) in order to accommodate the public's desire for both semi-primitive and developed recreational opportunities.

[Information on the Francis Marion's forest plan revision process can be found at the Francis Marion Plan Revision webpage: Francis Marion and Sumter National Forests - Planning (usda.gov).]

Colville National Forest. The Colville National Forest is situated in the sparsely populated northeast corner of Washington State. The forest is part of the ecologically diverse Columbia Highlands. Timber production and grazing have been (and still are) 
important forest uses, but the forest also supports a wide variety of flora and fauna, including several species protected under the Endangered Species Act. The forest also contains over 200,000 acres of old-growth forests, a critical habitat for the endangered spotted owl. Regulations regarding protection of these old-growth forests fall under the regionally-focused Northwest Forest Plan of 1994. Hundreds of miles of forest roads and trails provide access to both developed and backcountry areas that are popular for gathering forest products, nature viewing, hunting and fishing, hiking, camping, white water rafting, mountain biking, rock climbing, cross-country skiing, horseback riding, snowmobiling and OHV use. Management of the Colville has often been challenging and often contentious, due to the diversity of landscapes and private and governmental interests at play.

The Colville began its plan revision process in 2004 under the 1982 planning rule and was plagued with the same fits and starts as the Coconino as various Forest Planning Rule revisions were making their way through the courts. The Colville's record of decision was submitted in late 2019. However, Colville study participants felt that navigating 15 years of the plan revision process was made smoother by adopting a formal business plan that laid out the operational mechanics of the process and who would be responsible for what decisions. ${ }^{5}$ Participants felt this business plan was instrumental in maintaining a consistent planning process even given the leadership and workflow disruptions that resulted from multiple staff turnovers during the long effort. Participants

\footnotetext{
${ }^{5}$ The date that this business plan was instituted was not provided by participants and the business plan is not discussed in publicly accessible webpages (it is an internal operating document), but was likely constructed around the time the Colville separated its plan revision process from the adjacent OkanoganWenatchee forest in 2014 (the two forests had originally combined their plan revision efforts).
} 
also mentioned that utilization of this business plan improved relationships with communities, increased public involvement in and acceptance of forest management decisions, and ultimately decreased overall operational costs. As evidence, Colville participants report that the business model has been highly effective at the project-level as well. For example, the forest has moved 22 timber-related projects forward without appeal or litigation.

The Colville also contracted with an external facilitator to develop and manage the public participatory process. Participants indicated that outside facilitation helped to insert the skillsets needed for effective public outreach and engagement that were not available internally and lessened the burden on technical and specialist staff. The Colville used maps prolifically in their public outreach which included an interactive online mapping webpage displaying layers on various issue areas, such as recreation, wilderness, etc. In several public information sessions, facilitators placed maps on the walls where the public could indicate their recreational values and use preferences by drawing on these maps. The conversations that occurred and insights that were gleaned during these types of activities helped the ID team better understand and balance competing land-use preferences which they felt led to greater public understanding and acceptance of the draft plan alternatives.

[Information on the Colville's forest plan revision process Forest can be found at the Colville Plan Revision webpage: Colville National Forest - Planning (usda.gov).]

\subsection{Participant Descriptors}

Participant recruitment and interviews were conducted over approximately one year from March, 2015 through May, 2016. Interviews ranged from 30 minutes to over two hours, averaging about one hour each; 20 interviews were conducted by phone; six 
interviews in-person. In total, 26 interviews were completed and transcribed for this study including 13 forest-level staff, five regional office staff, five research station staff and three academic researchers. Table 4 compiles the descriptors for the 23 USFS participants in this study including the number of service years (in the USFS) and educational background.

Table 4: Study participant descriptors.

\begin{tabular}{|c|c|c|c|c|c|c|}
\hline \multirow[b]{2}{*}{$\begin{array}{c}\text { Agency } \\
\text { Level }\end{array}$} & \multicolumn{3}{|c|}{$\begin{array}{l}\text { \# Service Years } \\
\text { (at USFS) }\end{array}$} & \multicolumn{3}{|c|}{$\begin{array}{c}\text { Education } \\
\text { (degree or area of study) }\end{array}$} \\
\hline & $\begin{array}{l}\text { High } \\
16+\end{array}$ & $\begin{array}{l}\text { Med } \\
6-15\end{array}$ & $\begin{array}{c}\text { Low } \\
1-5\end{array}$ & Natural Science & Social Science & Practitioner \\
\hline Forest & 7 & 5 & 1 & 5 & 3 & 5 \\
\hline Regional & 2 & 3 & 0 & 0 & $4^{*}$ & 1 \\
\hline \multirow[t]{10}{*}{ Research } & 4 & 1 & 0 & 0 & $5 *$ & 0 \\
\hline & 13 & 9 & 1 & & & \\
\hline & & & & Forestry & Economics & Landscape Architect \\
\hline & & & & Silviculture & Recreation/Tourism & Public Affairs \\
\hline & & & & Hydrology & Resource Mgmt. & Environmental Law \\
\hline & & & & Wildlife Biology & Anthropology & \\
\hline & & & & Zoology & Geography & \\
\hline & & & & $\begin{array}{l}\text { Environmental } \\
\text { Science }\end{array}$ & & \\
\hline & & & & Soil Science & & \\
\hline & & & & \multicolumn{3}{|c|}{$\begin{array}{l}\text { *Only social scientists at the Research Stations and Regional } \\
\text { Offices were recruited. }\end{array}$} \\
\hline
\end{tabular}

Summary. The outreach strategy to USFS participants reached employees with many years of experience. Over $57 \%$ of the participants had been with the USFS over 15 years at the time of the study and had been through at least one prior plan development process and numerous amendment submissions before the current effort. An additional $39 \%$ had been with the USFS over 6 years. While the study specifically targeted social scientists, particularly at the research stations and regional offices, five forest-level 
participants have degrees or areas of study in the natural or biophysical sciences. Six other participants self-identify as practitioners, which include landscape architecture, public affairs and environmental law.

While it is customary to provide details about who was interviewed, from where, and individual subject specialties, providing such detail would violate the confidentiality agreement provided to participants. Because only 23 interviews were conducted, spread among staff at the forest level, regional offices and research stations, it is likely that such detailed information would allow a reader to easily identify individual study participants. The distribution of participant characteristics provided in the table above shows that most of the study participants were highly experienced with a long tenure at the USFS and represent 15 different areas of specialty. As will be detailed in succeeding chapters, the data provided by these participants represents a wide variety of well-informed perspectives on forest planning. 


\section{DATA ANALYSIS STRATEGY}

\subsection{Applied Thematic Analysis}

The analysis approach adopted in this study allows research findings to emerge from the data through applied thematic analysis. Guest et al. (2012) define applied thematic analysis as "a rigorous, yet inductive, set of procedures designed to identify and examine themes from textual data in a way that is transparent and credible" (Guest et al., 2012, p. 15). As shown in Figure 6, the steps in analyzing textual data follow a pattern of moving from what is likely many pages of narrative through an iterative process that segments the text, identifies common (salient) themes within those segments, then derives meaning from those themes.

Figure 6: The process of applied thematic analysis.

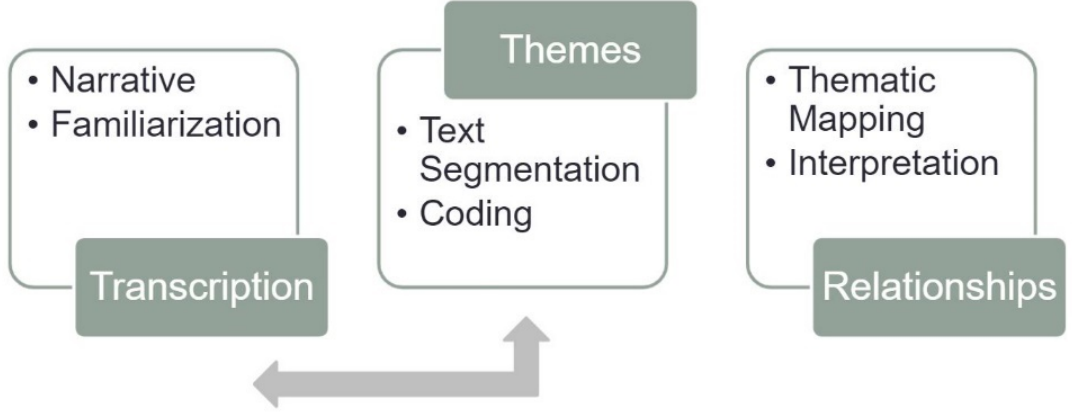

Source: Adapted from Guest et al., 2012.

The applied thematic analysis adopted in this study follows this iterative process and can be described in four steps: (1) identifying segments of text using a set of structural codes (also called tags or labels) related to the four initial conceptual framework categories; (2) using the first coding exercise to examine the text and develop a comprehensive set of related sub-codes that identify factors within the broad categories and then applying those sub-codes to the text; (3) refining and reducing the codebook 
through additional readings of text segments, removing sub-codes that seem irrelevant and combining similar sub-codes where appropriate; and (4) the final reading of text segments to identify predominant themes related to the research question. This final step includes incorporation of additional analytical tools - analytic memos to consolidate and summarize participant comments and cut-and-sort techniques to parse text segments into like topical categories. The intended outcome of the process is to create a small number of summary groups that provide context and identify themes that represent salient challenges and opportunities in collecting and using socio-spatial data as identified by study participants.

\subsection{Coding Methodology}

First Level Coding (Verifying Parent Codes). All interviews were recorded with permission and transcribed. Using an online qualitative research tool (Dedoose $\odot$ ), the transcriptions were uploaded and coded soon after the interview using a technique referred to as structural coding. Structural coding is a method that tags segments of data driven by specific research objectives and is particularly appropriate for studies employing multiple participants, standardized or semi-structured data-gathering protocols and exploratory investigations (Guest et al., 2012; Gibbs, 2012; Namey et al., 2008; Saldana, 2013). As MacQueen et al. (2008) note, "structural coding generally results in the identification of large segments of text on broad topics; these segments can then form the basis for an in-depth analysis within or across topics" (MacQueen et al., 2008, p. 125). In structural coding, applying quantitative tools is also possible to aid in developing coding structures, such as determining code frequencies and co-occurrences of code labels. 
In the first iteration of coding, I developed and applied a parent (first level) coding structure to the text based on the categories identified in the initial conceptual framework. No attempt was made at this stage to look for nuances, patterns or relationships in the data. The intent of this process was to gauge whether the interview protocols were homing in on categories relevant to the study and the extent to which participants were responding.

After coding the first few transcripts, Data Collection was added as a parent code (originally subsumed in the generalized Planning or Science code depending on context) as participants frequently spoke in detail on this topic. The initial Perceptual parent code (related to spatial literacy and comfort with social science concepts and protocols) was removed. Responses to questions around this topic indicated no challenges or barriers or any other distinguishing factors, at least within this dataset. ${ }^{6}$ While participants did talk about challenges in interdisciplinary work (between natural and social scientists, which is covered in the Science code), their comments indicated ample knowledge about social science and geospatial data. Table 5 below lists the seven parent codes with a code description and example text. As a note, the example texts often indicate possible linkages between codes (e.g. organizational structure and organizational culture). These linkages are examined at later stages using thematic networking.

\footnotetext{
${ }^{6}$ It should be noted that the purposive participant selection process targeted social scientists, so it is no surprise that they were well versed in social science methods and data. However, participants included numerous natural and biophysical specialties as well. These participants also expressed good knowledge of social science as well as an understanding of the nature of socio-spatial data.
} 
Table 5: First level parent codes with description and example text.

\begin{tabular}{|c|c|c|}
\hline $\begin{array}{l}\text { PARENT } \\
\text { CODE }\end{array}$ & DESCRIPTION & EXAMPLE TEXT \\
\hline EXTERNAL & $\begin{array}{l}\text { References to federal laws, regulations and } \\
\text { rules that represent the legal framework in } \\
\text { which USFS operations occur, such as } \\
\text { NEPA, ESA, OMB, MUSY or NFMA; } \\
\text { comments about the } 1982 \text { or } 2012 \text { Forest } \\
\text { Planning Rule, references about external } \\
\text { political forces, either overt or covert, such } \\
\text { as "litigation or conflict mentality," } \\
\text { "policy-makers," "Congress" or "public } \\
\text { sentiment." } \\
\text { DO NOT use for references to internal } \\
\text { USFS standard operating procedures (use } \\
\text { ORG STRUCTURE).. }\end{array}$ & $\begin{array}{l}\text { External Protocols (PPGIS) and Laws } \\
\text { (OMB): Part of it [forest planning] is } \\
\text { figuring stuff out for the first time. What I } \\
\text { mean by that is things like a PPGIS } \\
\text { protocol. Clearly it brings a lot of value to } \\
\text { the forest plan revision process. But, } \\
\text { corporately there's not a lot of organization } \\
\text { about how to do that, how to steer clear of } \\
\text { OMB requirements. You know we need this } \\
\text { OMB clearance. Oh my god, if I could ask } \\
\text { for one thing it would be that! }\end{array}$ \\
\hline $\begin{array}{l}\text { ORGANIZATIONAL } \\
\text { STRUCTURE }\end{array}$ & $\begin{array}{l}\text { References to how roles, responsibilities } \\
\text { and authorities are defined, assigned, } \\
\text { controlled and coordinated within the } \\
\text { agency to achieve organizational goals. } \\
\text { Includes references to leadership, decision- } \\
\text { making, budgets, staff incentives, } \\
\text { communication strategies and standard } \\
\text { operating procedures. }\end{array}$ & $\begin{array}{l}\text { Standard Operating Procedures and } \\
\text { Planning Strategy: That's why I think } \\
\text { geographic areas are so important...It [the } \\
\text { land] becomes the common denominator by } \\
\text { which we can all get around the table and } \\
\text { force the integration...The management } \\
\text { area approach, managed by purpose, allows } \\
\text { us to retreat back to individual } \\
\text { priorities... We're managing for wildlife; } \\
\text { we're managing for fire; we're managing } \\
\text { for rec... Well, all of those purposes may } \\
\text { happen within that place. }\end{array}$ \\
\hline $\begin{array}{l}\text { ORGANIZATIONAL } \\
\text { CULTURE }\end{array}$ & $\begin{array}{l}\text { References to the shared assumptions, } \\
\text { values, and beliefs which govern how } \\
\text { employees behave in the agency, } \\
\text { particularly how they are expected to } \\
\text { interact and perform their jobs. Includes } \\
\text { accepted ways of behaving and/or habits } \\
\text { (e.g. habits of planning; culture of } \\
\text { science/expertise). These are comments } \\
\text { indicating "this is how we are expected to } \\
\text { do our job" as long as it does NOT } \\
\text { specifically relate to formalized operating } \\
\text { procedures. }\end{array}$ & $\begin{array}{l}\text { Culture of Science: To me that comes to the } \\
\text { point where data becomes less important } \\
\text { than just putting some basic rules into place } \\
\text { about how you go about planning... That's a } \\
\text { hard thing for this agency to deal with - } \\
\text { that thought - because we re very much } \\
\text { scientists and we want to have everything } \\
\text { perfected on that front. It's hard to get all } \\
\text { of these resource specialists to let go of } \\
\text { that...The public gets it. It's internally - the } \\
\text { agency specialists - that struggle with it. I } \\
\text { say, we're gonna get stuff wrong... but it's } \\
\text { how they frame it in their heads...we get } \\
\text { paralyzed by this stuff and it's all internal! }\end{array}$ \\
\hline PLANNING & $\begin{array}{l}\text { References to forest planning strategies, } \\
\text { processes, protocols, rules, timing; } \\
\text { perceived challenges and opportunities in } \\
\text { forest planning; participant involvement in } \\
\text { and opinions about the planning process; } \\
\text { descriptions of planning documents. } \\
\text { DO NOT include references to how the } \\
\text { public is engaged in this process or how } \\
\text { data is collected and/or used. Use PUBLIC }\end{array}$ & $\begin{array}{l}\text { Planning Challenges: This also happened } \\
\text { with our draft EIS as well...A lot of the } \\
\text { reviews required some updates and when } \\
\text { you change one thing it kind of ripples } \\
\text { through the document...You know it's } \\
\text { months...it's months...six months plus. So I } \\
\text { don't think there's full appreciation for the } \\
\text { complexity in forest plan revisions and what } \\
\text { that means when you have to revisit a topic } \\
\text { again and again and make adjustments. }\end{array}$ \\
\hline
\end{tabular}




\begin{tabular}{|c|c|c|}
\hline & $\begin{array}{l}\text { ENGAGEMENT or DATA } \\
\text { COLLECTION code. }\end{array}$ & \\
\hline $\begin{array}{l}\text { PUBLIC } \\
\text { ENGAGEMENT }\end{array}$ & $\begin{array}{l}\text { References to public outreach, } \\
\text { participation in the planning process } \\
\text { (including involvement of collaboratives), } \\
\text { communication with and dissemination of } \\
\text { information to the public around forest } \\
\text { plan revision, participatory processes put } \\
\text { in place (or wished to have been put in } \\
\text { place) during the planning process. }\end{array}$ & $\begin{array}{l}\text { We shouldn't be there to tell them [the } \\
\text { community] what a healthy community is. } \\
\text { They should be telling us. And that way } \\
\text { there is some shared vision over that...So } \\
\text { it's interesting to be more engaged. You } \\
\text { know, we're pretty insulated...So I think it } \\
\text { would be better if we had done some of that } \\
\text { community sensing and those community } \\
\text { connections earlier. }\end{array}$ \\
\hline DATA COLLECTION & $\begin{array}{l}\text { References to the collection and/or use of } \\
\text { social-cultural-economic or socio-spatial } \\
\text { data in the planning process, assessments, } \\
\text { plans, and DEIS/FEIS. This could also } \\
\text { refer to specific data collected and tools } \\
\text { used (e.g. PPGIS, LVM, MPLAN, } \\
\text { NVUM, WVM, etc.); references to data } \\
\text { gaps particularly as it relates to socio- } \\
\text { spatial data. }\end{array}$ & $\begin{array}{l}\text { Well, taking the recreation one where we } \\
\text { asked people to identify their special places, } \\
\text { I think that is a hit and a miss with that, } \\
\text { maybe in just the way we did it. We had } \\
\text { maps on the wall and we said, "Just draw a } \\
\text { circle around your favorite places and tell } \\
\text { us what recreational activity you do in that } \\
\text { place." And people either drew great big } \\
\text { huge circles or they did a dot. So the } \\
\text { usefulness, sort of like the rigor of that } \\
\text { information, may not have been really } \\
\text { great. }\end{array}$ \\
\hline SCIENCE & $\begin{array}{l}\text { Broad references to science including the } \\
\text { role of science in the participant's job, } \\
\text { collaboration and networking among and } \\
\text { between scientists and/or practitioners; } \\
\text { descriptions or opinions about where } \\
\text { science (social and/or natural) fits into the } \\
\text { planning process, Best Available Science } \\
\text { (BAS), science synthesis, and research } \\
\text { agendas. } \\
\text { DO NOT use for specific references to } \\
\text { data or data collection. Use the DATA } \\
\text { COLLECTION code. }\end{array}$ & $\begin{array}{l}\text { Science Synthesis: We led a collaborative } \\
\text { effort where we brought a lot of } \\
\text { stakeholders together to have conversations } \\
\text { with researchers around the most pressing } \\
\text { science-based questions... they actually } \\
\text { condensed it into a single question...how do } \\
\text { you in fact move towards social, economic } \\
\text { and ecological sustainability? The new } \\
\text { science comes in where the researchers } \\
\text { pulled together existing literature that } \\
\text { addressed those different components. But } \\
\text { the new science is they [the researchers] } \\
\text { had to work together to integrate it all to } \\
\text { answer a new question, right? }\end{array}$ \\
\hline
\end{tabular}

In addition to the seven parent codes, descriptors were also applied to the dataset.

These include EDUCATION, CAREER, SERVICE and LEVEL. Initial questions asked

participants about their educational background and areas of academic study, their career

trajectory within the USFS (how many assignments and where), and number of service

years. The LEVEL code distinguishes participants by Forest, Regional Office, Research

Station or Scholar. These descriptors are used to examine the characteristics of the

participants and the potential for variance in responses based on these descriptors. 
First level coding helps to gain familiarity with the dataset and can be used to determine whether a sufficient number of text segments have been collected representing the primary categories of interest. Because semi-structured interviews allow the participant to respond to a prompt without much interruption, segments within the narrative often cover several topics and may be tagged with multiple codes. Text segments at this coding level can be as small as a few sentences or extend over a page or more in the transcript. Table 6 displays a frequency chart exported from Dedoose $\odot$ that indicates the total number of text segments tagged with first level codes and the average per participant by the LEVEL descriptor. The frequency of a code assignment does not provide any information about the nature of the comments, and it should be noted that prompting questions in the interview protocols attempted to address all these first level codes. It does show, however, that participants have something to say about each of the primary categories of interest to varying degrees.

Table 6: Aggregate number of coded segments by first level code and organization level.

\begin{tabular}{|c|c|c|c|c|c|c|c|c|}
\hline LEVEL & EXTERNAL & STRUCTURE & CULTURE & PLANNING & PUB ENG & DATA & SCIENCE & \\
\hline \multirow[t]{2}{*}{ Forest } & 16 & 155 & 23 & 169 & 118 & 90 & 69 & Total \\
\hline & 1.2 & 11.9 & 1.8 & 13.0 & 9.1 & 6.9 & 5.3 & Average \\
\hline \multirow[t]{2}{*}{ Regional } & 17 & 101 & 16 & 48 & 43 & 49 & 25 & Total \\
\hline & 3.4 & 20.2 & 3.2 & 9.6 & 8.6 & 9.8 & 5.0 & Average \\
\hline \multirow[t]{2}{*}{ Research } & 22 & 57 & 13 & 42 & 7 & 49 & 38 & Total \\
\hline & 4.4 & 11.4 & 2.6 & 8.4 & 1.4 & 9.8 & 7.6 & Average \\
\hline \multirow[t]{2}{*}{ Scholars } & 36 & 60 & 23 & 25 & 20 & 37 & 21 & Total \\
\hline & 12.0 & 20.0 & 7.7 & 8.3 & 6.7 & 12.3 & 7.0 & Average \\
\hline \multirow[t]{2}{*}{ TOTAL } & 91 & 373 & 75 & 294 & 188 & 225 & 153 & Total \\
\hline & 3.5 & 14.3 & 2.9 & 10.9 & 7.2 & 8.7 & 5.9 & Average \\
\hline
\end{tabular}

There are no definitive rules around how many text segments are sufficient for qualitative data analysis. That being said, there are two first level codes with close to or over 300 tagged text segments, well over 100 segments coded for three additional codes, 
and close to that number for the remaining two codes. Text segments were extracted from over 300 single-spaced pages of interview transcriptions. At the point of completing 26 interviews, I made the determination that the large number of text segments across the full spectrum of parent codes indicated there was a sufficiently comprehensive dataset to proceed to the next level of coding.

The frequency chart is also a useful tool to assess the relevance of the codes within the context of the research question and guide the next stage in the coding process. For example, using the average per participant (which is simply the total segments divided by the number of interviewees in that functional level), organizational structure garnered the highest number of responses across levels and warranted a more nuanced look in ensuing coding activities to tease out what aspects of organizational structure prove to be the most relevant.

Second Level Coding (Refining the Codebook). The second iteration of coding in applied thematic analysis is a critical sorting activity that parses the data into more refined text segments. This coding level untangles large blocks of text to capture nuances in the data. I developed a set of 33 sub-codes related to the parent codes by identifying keywords for factors proposed in the initial conceptual framework, reviewing the summary field notes to uncover additional compelling factors, and adding sub-codes as they emerged from reading the transcripts. Table 7 lists the 33 sub-codes under their respective parent codes. Appendix H provides a detailed list and description of the subcodes. 
Table 7: Parent structural codes and second level sub-codes.*

\begin{tabular}{|c|c|c|c|}
\hline $\begin{array}{l}\text { EXTERNAL } \\
\text { Public Policy } \\
\text { Litigation } \\
\text { OMB } \\
\text { NEPA } \\
\text { FACA }\end{array}$ & $\begin{array}{l}\text { ORGANIZATIONAL } \\
\text { CULTURE } \\
\text { Behavior } \\
\text { Habits }\end{array}$ & $\begin{array}{l}\text { PUBLIC ENGAGEMENT } \\
\text { Sense of Place } \\
\text { Outreach } \\
\text { Information } \\
\text { Trust } \\
\text { Collaboration }\end{array}$ & $\begin{array}{l}\text { PLANNING } \\
\text { Planning Rule } \\
\text { Planning Process } \\
\text { Timing }\end{array}$ \\
\hline $\begin{array}{l}\text { DATA } \\
\text { COLLECTION } \\
\text { Spatial Data } \\
\text { Data Issues } \\
\text { Data Tools } \\
\text { Mapping }\end{array}$ & $\begin{array}{l}\text { SCIENCE } \\
\text { Research } \\
\text { BASI } \\
\text { Paradigms } \\
\text { Networking }\end{array}$ & $\begin{array}{l}\text { ORGANIZATIONAL } \\
\text { STRUCTURE } \\
\text { Integration (roles) } \\
\text { Infrastructure } \\
\text { Strategy (SOP) } \\
\text { Capacity } \\
\text { Budget } \\
\text { Leadership } \\
\text { Decision-Making } \\
\text { Communication } \\
\text { Incentives } \\
\text { Resilience }\end{array}$ & $\begin{array}{l}\text { QUOTES } \\
\text { This tag was used to } \\
\text { highlight specific } \\
\text { text segments that } \\
\text { seemed particularly } \\
\text { germane to the } \\
\text { categories and } \\
\text { factors of interest to } \\
\text { be used for reporting. }\end{array}$ \\
\hline
\end{tabular}

*See Appendix $\mathrm{H}$ for a detailed list and description of each sub-code.

For the second iteration of coding, I reread all the transcripts and liberally assigned the set of sub-codes to the text. Ryan and Bernard (2003) refer to this technique as "eyeballing" or "pawing." It allows the researcher to parse the entire dataset into more nuanced segments while gaining a great deal of familiarity with the dataset as a whole. Exclusivity is not a goal in this step. Large text segments often have multiple overlapping codes that begin to reveal patterns and relationships. The outcome is a dataset with codes assigned to all relevant text. This step is also used to locate and tag any questionable or inappropriate text segments within the context of the study. These kinds of text segments were not ubiquitous in this dataset. The study prospectus, email invite and consent form made it clear that participants would be interviewed within their capacity as agency employees; all participants conducted themselves in a professional manner. I did tag, and ultimately removed, several pages of text in one transcript that digressed significantly from the primary purpose of the interview. I also removed tags 
for a few text segments in two other interviews in which the participants followed the comment with statements akin to "oops...you probably should not include that!"

This coding step is not linear, but rather iterative. For example, as I was reading and coding the transcripts, I added several sub-codes to the Organizational Structure parent code in order to make visible the diversity of comments I was seeing in the text. I also clarified the definition of several sub-codes in the Data Collection parent code to remove overlap that was causing inconsistencies in segment coding. Consequently, it was necessary to go back to the beginning several times and assess and assign new or revised sub-codes to previously reviewed transcripts. While indeed time-consuming, the entire dataset was reviewed several times, meticulously coded several times, and, as a perk, consistent themes in the dataset began to emerge and percolate.

Final Coding (Selecting the Most Relevant Codes). The task in the third iteration of coding involves determining which sub-codes can be combined (reflecting similar comments) or eliminated (if the sub-code is used infrequently). The goal is to reduce the total number of sub-codes by at least $50 \%$ through a careful review of the text segments assigned to each code within the context of the study objectives (Guest et al., 2012). This process is much like constructing an executive summary. While the detail of the full dataset is always available to refer back to, the purpose of this consolidation step is to condense a large body of information into what are deemed the most important, insightful and explanatory points relative to the research objectives. I approached this challenge using two simultaneous processes as described below.

Using a Dedoose $C$-generated frequency chart of the assigned sub-codes, I assessed the number of text segments assigned to each code and identified sub-codes that 
had 30 or less segments (each representing less than $2 \%$ of over 1,700 total segments with assigned sub-codes). This resulted in reassessment of 16 of the 33 sub-codes using frequency alone. To reiterate, the frequency of a code assignment does not reveal anything about the relevance of the text, but it does indicate that the sub-codes did not represent a plethora of responses. These were not factors in which respondents necessarily gravitated toward despite prompting or probing questions or the sub-code might have been irrelevant, ill-defined or could be reasonably merged with another subcode without losing important insight.

Simultaneously, I applied a critical lens in making decisions about sub-code suitability and possible text reassignment. In reading the text segments for each of the sub-codes, I posed a set of questions: (1) what is the main argument or point of this segment; (2) to what extent do the main points directly address the research question and objectives; and (3) are there any new insights evident in this text segment and, if so, can these insights be captured by another related code or should they remain intact? In all cases, I reviewed the text segments within the context of the full transcript before deciding to merge or eliminate a sub-code so that I avoided de-coding an isolated block of narrative or merging sub-codes in a way that would obscure emerging themes.

In this coding step, nuance was retained in the Organizational Structure parent code using four final codes, as this category occurred at the greatest frequency and in cooccurrence with other codes. The Science parent code retained two final codes to maintain the qualitative difference of references in these parent codes (relationships between scientists as opposed to definitions of BASI). The sub-codes in the External Policy \& Law, Organizational Culture, and Public Engagement parent codes were 
merged into one final code each. The Planning parent code was removed as unnecessary; all interviews were conducted within the context of the forest plan revision process, so this code ended up simply catching general comments on the mechanics of forest planning and lacked insight into specific integration of socio-cultural and socio-spatial data into the process. Initially, the Socio-Spatial Data and General Data codes were kept separate. However, during the cut-and-sort process (that is described in the next section), the Data General code was further parsed into themes more relevant to the Socio-Spatial Data code (in particular, scale and data gaps). These segments were subsumed into the Socio-Spatial Data Code and the Data General code was eliminated.

The final code list contains ten descriptive codes. The text from the eliminated sub-codes were merged into their reassigned final code using Dedoose $\odot$ and, in a final reading of the transcripts, the final code assignments for all text segments were reviewed. The final codes are listed below. Code descriptions and example text segments for these final codes can be found in Appendix I.

Final codes include:

1. Organizational Structure (roles \& responsibilities, communication, standard operating procedures, incentives)

2. Organizational Capacity (staffing/turnover, expertise, adaptability/resilience)

3. Organizational Leadership (authority, decision-making)

4. Organizational Budget

5. Organizational Culture

6. External Policy \& Law

7. Public Engagement

8. Science Networking

9. Science/BASI

10. Socio-Spatial Data

In thematic analysis, coding activities should occur without preconceived notions about what is relevant and what is not (interpretation occurs after coding). However, 
there are a few insights and preliminary conclusions that can be garnered from this final coding process. The perceptual category in the initial conceptual framework, that focused on comfort levels with socio-cultural data in forest planning and socio-spatial literacy (particularly around values mapping), proved insignificant in this particular dataset. Both social and natural scientists that were interviewed understood the nature and value of sense of place data and most offered detailed descriptions on how sociospatial data had been gathered and incorporated into their planning process. The challenges around collecting socio-spatial data seemed to be directly related to structural factors, as well as external contingencies, that made it difficult to prioritize and/or collect this kind of data. It should be noted that this may be due to the participant sampling strategy that targeted potential study participants from the ID teams brought together for the plan revision process. An in-depth understanding of the nature of socio-spatial data may not be as evident for natural and physical scientists that work in specific program areas at the regional offices that are not routinely exposed to such interdisciplinary work.

\subsection{Developing Themes}

The next stage in applied thematic analysis moves from coding the entire dataset to deriving meaning from it through the identification and interpretation of a set of persistent themes that emerge from the data. This is the stage in which all the data is reviewed with a laser focus on the research question and objectives. At this point it is useful to briefly discuss the difference between codes and themes in thematic analysis.

Codes can be best described as labels (also referred to as tags) that group similar textual references. Codes are used to extract text that is relevant to the research question and eliminate that which does not apply (Gibbs, 2012). The codes themselves are not 
particularly revealing, although addition and elimination of codes during the iterative coding process may indicate the efficacy of categories and associated factors included in an initial conceptual framework. For example, this study's initial conceptual framework includes a Perceptual category which is not validated by participant comments and therefore does not seem relevant for this dataset.

Codes organize the data - answering the question, what is important to focus on in this dataset? Themes, on the other hand, are the insights gleaned from the actual text attached to each final code, capturing what is revealed that has explanatory power related to the research topic. Vaismoradi and Snelgrove (2019) describe themes as the "underlying meanings within which similar pieces of data can be tied together and within which the researcher may answer the question, why?" (Vaismoradi \& Snelgrove, 2019, p. 2). Themes can be quite specific, referring to an identified causal factor (such as a particular law or regulation), or more general (such as a clearly articulated challenge that permeates the text, but may affect participants or systems in different ways under different circumstances).

A natural question at this point is: what constitutes a theme? This is partially a question of prevalence of similar comments within a final code. When looking for themes, confidence in a theme's relevance is increased the more times the theme appears among study participants and in different contexts. However, more instances of specific comments do not necessarily mean the theme itself is more enlightening or meaningful. As Braun and Clarke (2006) note, "in qualitative analysis, there is no hard-and-fast answer to the question of what proportion of a dataset needs to display evidence of the theme for it to be considered a theme... the 'keyness' of a theme is not necessarily 
dependent on quantifiable measures, but rather on whether it captures something important in relation to the overall research question" (Braun \& Clarke, 2006, p. 82). What is important is the ability of the theme to capture particularly informative or explanatory aspects about the phenomenon of interest.

In qualitative analysis, the researcher determines what themes are important and becomes the primary interpreter of the dataset. That a fair amount of subjectivity is applied in this process must be recognized. However, as is the case for all research, transparency around how decisions are made at all steps in the process - data collection, coding, thematizing, interpreting - allows other researchers to gauge the rigor of the study design and the validity of the interpretation. Application of several qualitative analysis tools to develop themes appropriate to the study goal also mitigates subjectivity by allowing for triangulation of results. Toward that goal, I selected three analysis tools that are recommended for studies seeking meta-themes (statements that reflect a broad meaning behind a set of similar insights). Each tool explores the data using a slightly different process or set of questions. The analyses work in tandem to contextualize, identify and elucidate the most persistent themes within and across the dataset. Confidence in the salience of the identified themes is increased when consistently verified across different analyses. The following sections describe the tools used for each of the analytical exercises.

\subsection{Analytic Memos}

The analytic memo is a structured means by which to question, document and reflect on the effectiveness of the coding process, examine how the process of inquiry is taking shape, and to identify perspectives, opinions and insights in the data that directly 
speak to the research objectives (Thomas, 2006). The Analytic Memo applied in this study asked the following questions for each interview:

1. What seems most important or significant to this participant? What stood out most in the interview?

2. How did this participant talk about the use of social science, socio-spatial data or data gaps? Was it said in a positive, negative or neutral context?

3. Was there anything in the interview that surprised you? What insights did you gain?

4. Did the participant talk about significant links, challenges or opportunities? What were they?

The first step consisted of checking final codes on each transcript. The goal was to heighten the level of confidence that the final coding structure adequately represented the transcript content, as these final codes would be used for the cut-and-sort exercise that is detailed in the next section. This was the final review undertaken within the context of the full interview transcript. In addition, attention was given to potential links and relationships within codes through additional tagging. Once a transcript had been fully reviewed, an analytic memo was prepared to consolidate important points within the transcript, noting the intensity (strong language used) and persistence (referred to often and in different contexts) of comments.

The analytic memos for each transcript were aggregated by the three administrative unit - forests, regional offices and research stations - with a summary meta-memo generated for each level. A final review of field notes for each interview also contributed to the meta-memo. The full Analytic Meta-Memo is attached as Appendix J. Individual analytic memos and field notes for the interviews will not be included here as they contain identifiable information. 
The analytic memo serves as both a consolidation and cross-check tool.

Information contained within each transcript is synthesized into an individual memo at the time of final coding that highlights key perspectives, opinions, insights and comments of each study participant. In this way, the tool helps to reduce information overload as it provides a mechanism to mentally close out one interview transcript and move on to the next. The analytic meta-memo (which consolidates all the individual memos) also serves as a reliability check for the themes that emerge from the cut-and-sort tool that is described below. Confidence in the relevance of the identified themes using cut-and-sort is increased if they are also reflected in the meta-memo.

\subsection{The Cut-and-Sort Technique}

The cut-and-sort technique (also called piling or stacking) is used to identify persistent themes in lengthy, verbatim text segments that are rich with content and potential relationships by arranging text segments into piles of similar content (Ryan \& Bernard, 2003). While the analytic memo determines the main points in an interview transcript and asks what is of importance to a single participant, the cut-and-sort technique compiles text segments by the final code and analyzes how multiple participants are talking about a single subject. Text segments may be of differing lengths, but each is reduced to its primary point and grouped with other similar segments.

There are many ways to apply criteria to sorting the segments depending on the approach and objectives of the study. Numerous fine-grained themes can be identified and refined if the cut-and-sort technique is applied early and repeatedly during the coding process. This is often the case for research using a grounded theory approach. In this study, I was interested in identifying overarching or meta-themes and have chosen to 
apply this technique at the final stage of data analysis, extracting the most persistent themes after the dataset had already been reviewed and organized through numerous coding exercises. The text segments were evaluated with a focus on the research question and how the text represented, addressed, explained or contextualized barriers and opportunities around the collection and use of socio-spatial data.

Text segments were extracted from Dedoose $\mathcal{C}$, filtered by the final code assigned to the segment. A particular text segment may be tagged with several final codes that represent potential co-occurrences or links. In these cases, text segments are included in each of the code groups. For example, if a text segment is coded with both the Organizational Capacity and the Spatial Data tags, the text segment was included in each of those subsets. A word processor was used to compile and organize the text segments, using the commenting feature to add a descriptive label to like segments. For example, in the Organizational Capacity code set, "Turnover" was used as a criterion to group text segments that discussed the effects of departing staff. Use of the commenting tool was also helpful in inserting additional notes, such as whether turnover was considered a challenge or an opportunity in that particular text segment, and to note particularly strong associations with other codes.

Identifiers were not removed from the text segments. I wanted to make sure that a theme was not created using the comments of only one participant who chose to speak at length about a particular topic or returned to it at numerous points in the interview. While the individual comment might have been interesting, meta-themes should reflect consistency of responses from numerous participants (Ryan \& Bernard, 2003). Retaining 
the identifiers also allowed for comparison of comments made by participants in the different administrative units of the organization.

Once organized, groups of text segments were reviewed to look for commonalities in participant comments, references to causal factors, impacts or outcomes within the text, and general respondent points of view or opinions around the topic. The categorized information was then reduced to a thematic statement intended to capture the central idea evident in the text group. Each theme will be discussed in detail in Chapter 7, with representative samples of participant comments, drawing from a final database containing 213 text segments.

The cut-and-sort exercise is also used to comment on links and relationships evident in the themes. Exploring and interpreting these linkages are accomplished through thematic networking, the final analytical tool applied to this dataset. The description that follows outlines the steps in which to construct a thematic network.

\subsection{Thematic Networking}

Applied thematic analysis works with large amounts of narrative data that can be quite complex and multidimensional. To derive meaning from this kind of dataset requires moving through an iterative and inductive process of contraction and expansion and back to contraction. Through the coding process, the text is grouped into general categories, disentangled (expanded) to look for nuances within the categories, regrouped again on the basis of the extent to which a text segment informs the research question, then expanded into a set of consistent themes. Creating a thematic network looks for linkages and relationships among themes and assumes that a rigorous coding methodology has already been applied to the dataset and the most salient themes have 
been identified. It starts where coding and thematizing end. The themes are grouped by an organizing criterion to reveal relationships among the themes. Theme relationships and linkages are then graphically displayed as an interconnected system and interpreted through the theoretical or conceptual lens that guides the research. The thematic network emphasizes both the structural elements evident in the theme and some basic connectivity between the elements. Once constructed, the thematic network visualizes a holistic system and serves as an illustrative tool to aid interpretation (Attride-Stirling, 2001).

The thematic network is built by categorizing themes as basic, organizing and global. Attride-Stirling (2001) explains this ordering process as follows:

Basic Themes: Basic themes are the premises evident in the text, the thematic statements that have been constructed from the dataset. These themes alone might reveal very little about the interconnections within the dataset as a whole, but when sorted and grouped represent an organizing theme.

Organizing Themes: Organizing themes are the second-order categories that take basic themes and organize them into clusters of like content. The organizing theme summarizes the cluster of basic themes so that they reveal the main idea of what is important about the cluster -- the central issue, challenge, assumption, idea, or purpose. Organizing themes, interpreted together, represent a global theme.

Global Themes: Global themes are the highest-level themes that encompass the central idea or message in the dataset as a whole. These themes group a set of organizing themes that together present a unified argument, position or assertion about the whole dataset. In this sense, global themes are both a summary of the main themes and a tool in which to frame an analysis - the primary premise. Standard symbology, such as bolded 
lines and arrows, are interpretive tools used to indicate relationships between elements and/or a directional impact (e.g. a feedback loop or causal agent). The thematic network displaying themes derived from this study can be found in Chapter 7.

Summary. Chapters 5 and 6 provide a detailed account of the data collection and analysis strategies employed in this research. Being transparent about how data was collected, coded and thematized allows for independent assessment of the rigor of the study design and appropriateness of the tools employed to collect and analyze the data. Each method and tool was selected to go through a step-by-step process to take the full breadth of the interview data and distill it into a set of meta-themes that can be constructed into a thematic network - a revised framework to situate the findings and inform practice and further research.

To this point, I have provided grounding in the background of the human dimensions of land-use planning (Chapter 1), a literature review culminating in a problem statement and research question (Chapter 2), information about theory that informed interpretation of the results and an initial conceptual framework that guided the study design (Chapter 3), and a detailed account of the case study, data collection and analysis strategies (Chapters 4, 5 and 6). I now turn to reporting the findings of this research and discussing implications for policy, practice and research. 


\section{FINDINGS}

This study began with a need to better understand and document how sociospatial data was being integrated into forest planning processes. The first insight derived from this study's analysis indicates that there is more to the collection and use of sociospatial data than perhaps current literature might suggest. On-the-ground planners are applying strategies to collect socio-spatial data in ways that inform decisions around forest plan components. However, much these efforts and impacts are embedded in public participatory processes during public sensing and scoping and are not explicitly documented in forest assessments or published documents.

To place, front and center, insights gained in how forest planners are integrating socio-spatial data, the discussion of the study's findings begins with four thematic statements around the spectrum of socio-spatial data collection and use. This is followed by 11 additional themes that identify salient factors informed by the initial conceptual framework (see page 35) and represent significant barriers, challenges and opportunities as identified by study participants. The purpose of the Findings chapter is to present the themes with a brief explanation and supporting quotations from the dataset. Insights that the themes evoke will also be presented, as well as compelling questions that suggest explicit or implicit linkages between themes or identify internal or external contingencies.

\subsection{The Spectrum of Socio-Spatial Data}

While this study focuses on a very specific aspect of forest planning - collection and use of socio-spatial data - it is impossible to divest socio-spatial data from the forest planning system as a whole. The process of creating the forest management plan is timeintensive, circumscribed and expensive (see Section 4.2 in Chapter 4). Each forest 
organizes and executes numerous information sessions, workshops, webinars, and public and private meetings to engage stakeholders and the public at large at numerous stages in the process. Data in many different forms is collected from the public at various stages and incorporated into the planning process to varying degrees. The following discussion of themes around the spectrum of socio-spatial data occurs within the context of this complicated planning system.

Text segments tagged with the Socio-Spatial Data code represent the most explicit narrative related to the collection and use of socio-spatial data These text segments are organized into four general thematic areas: (1) the diversity in types of socio-spatial data collection tools and data formats; (2) the application of these tools at various points in the plan revision process; (3) direct and indirect ways in which the data is used in decisionmaking; and (3) identified data gaps.

THEME: Socio-spatial data collection tools take many forms and produce different kinds of socio-spatial data.

It may seem obvious, but important to note nonetheless, that socio-spatial data is not something attached to a single tool. It is a specific category of data that makes tangible public values around places. Understanding factors that represent barriers and opportunities in the collection and use of socio-spatial data requires an appreciation of the many forms that socio-spatial data can take and the numerous tools which are used to collect this data throughout the lengthy planning process. Figure 7 distills the most common tools that are available to collect socio-spatial data on a continuum from simple to complex. The spectrum also provides a brief explanation on the process used to collect the data and the typical use of the data within the plan revision process. 
Figure 7: Socio-spatial data collection tools in forest planning.

\section{The Spectrum of Tools to Collect Socio-Spatial Data}

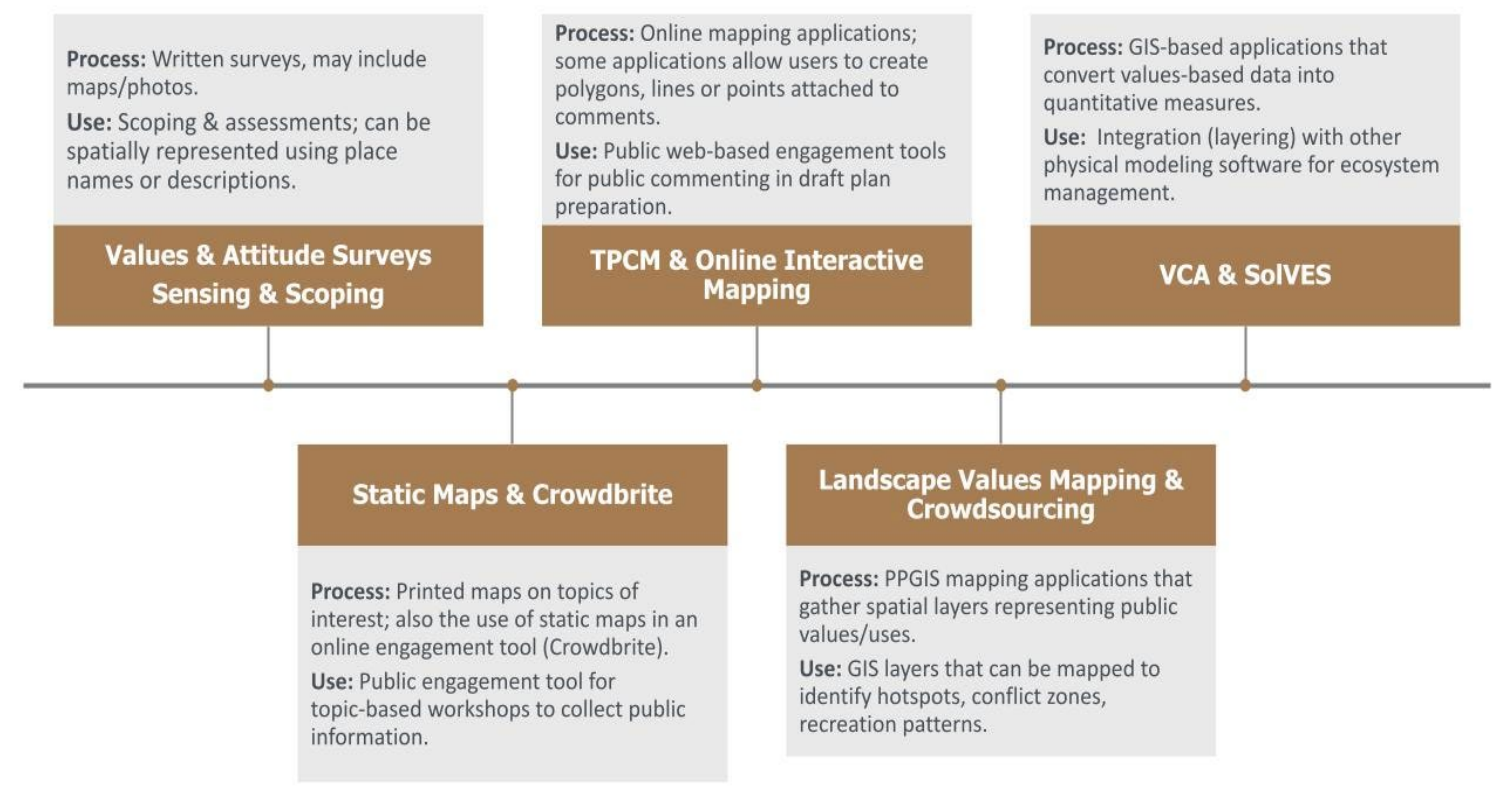

Some tools are less challenging to implement than others or more appropriate to employ at different stages in the public engagement strategy. For example, public sensing has been, and will continue to be, an active part of public engagement in forest planning, both in forest plan revisions as well as project-level planning. Public sensing consists of activities, such as information sessions or surveys, that reach out to the public to gauge public attitudes, values and preferences around an upcoming project or management task. Sense of place workshops using static or online maps represent a more targeted approach, also part of sensing and scoping, and often entail distilling general values and/or attitudes about specific places or management priorities (such as recreation use). Newer technology for gathering public input, such as the online Talking Points 
Collaborative Mapping (TPCM $)^{7}$ and other human ecology mapping tools and strategies provide more explicit spatially-referenced data and many produce GIS-ready layers that can be overlaid with other spatial data layers. While more sophisticated GIS-based applications that quantify values-based data for high-level spatial analysis are available (such as $\mathrm{VCA}^{8}$ or SolVES ${ }^{9}$ ), there is no evidence either in the academic literature, forest assessments or within study participant comments that these highly sophisticated tools have been used extensively on-the-ground by forest planners. There are significant challenges in both the legal and organizational spheres in using these sophisticated tools including the availability of primary GIS-ready socio-spatial datasets to input into these tools, the extent to which collecting the data is subject to OMB approval, and the level of expertise (or lack thereof) of USFS social science staff to gather, interpret and integrate this kind of data effectively.

Following are lists of opportunities and challenges that synthesize participant comments around the role of socio-spatial data in forest planning and challenges they have encountered. These opportunities and challenges provide a glimpse into how study participants view the purpose of socio-spatial data in forest planning. Comments highly correlate with public engagement, as would be expected when talking about public values-based data.

\footnotetext{
${ }^{7}$ TPCM is an online mapping application, developed through a partnership between the USFS and the U.S. Geological Survey, as a means to gather public input in planning, management and monitoring activities. The tool can track public comments on an online mapping interface (Aron \& Reed, 2015).

${ }^{8}$ Values Compatibility Analysis (VCA) is a decision-support system that gathers and operationalizes values-based data collected from the public into a digital format that can be used to compare public values with proposed management priorities (Brown \& Reed, 2012).

${ }^{9}$ SolVES was developed by the U.S. Geological Survey to incorporate quantified and spatially explicit measures of social values into standard ecosystem services modeling software (USGS Open-File Report 2015-1008: Social Values for Ecosystem Services, Version 3.0 (SolVES 3.0)-Documentation and User Manual)
} 
Opportunities:

- Mapping activities are fun and contribute to more effective and productive public outreach;

- Establishing "communities of place" through public mapping workshops is useful in understanding distinct groups of beneficiaries of forest services at varying scales and integrating that knowledge into planning documents;

- Mapping activities can help improve public buy-in for land management decisions;

- Public input into land management priorities is essential to comply with the USFS mission and is codified in the 2012 planning rule. This can incentivize investment in improving public engagement around attitudes toward forest management, in general, and the USFS, in particular.

Challenges:

- How socio-spatial data can add value to the planning process is not clearly documented;

- Designing public workshops then collecting, compiling and analyzing sociospatial data is often difficult due to staff capacity issues and/or time and budget constraints;

- Socio-spatial data generates questions about "whose data are we collecting?" Do workshops and online mapping applications attract only a self-selected constituency? How do we design better tools to reach a broader public?

Opportunities in using these tools indicate opinion along a spectrum from simply enhancing public engagement in forest planning to understanding how forest resources are beneficial to the public to reducing adverse public reaction to management decisions to ensuring legal compliance. Challenges indicate some organizational structural issues (capacity) as well as some uncertainty around what socio-spatial can (or should) do within the planning process, perhaps due to a lack of standardized protocols for its collection and use (e.g. there are no specific references to the use of socio-spatial data in the Forest Service Directives).

\section{THEME: Socio-spatial data collection tools are employed at different points in forest planning and for different purposes.}

The output of socio-spatial data collection activities rarely show up explicitly in formal forest assessments prepared during the initial plan revision process. The forest 
assessment of only one of the sample forests in this study (Francis Marion National Forest) included general references to socio-spatial data collected from public meetings. The Assessment Report does not display or cite any maps or data tables showing the socio-spatial data collected (although many were produced). However, Section 1.7.1 of the forest assessment includes a statement reflecting public values that reads: "At the public meetings, participants indicated that the Francis Marion was important because it provided opportunities to get away from the hectic pace of city life, to find peace and solitude, and to enjoy the natural environment in a number of ways, such picnicking, kayaking, fishing, hunting and bird watching" (Francis Marion National Forest, Draft Plan Assessment, 2013, p. 4).

In analyzing comments made by participants on ID planning teams, a lack of formal documentation does not mean this type of data is not used in informing forest management priorities, developing plan alternatives and making management decisions. All the sample forests in this study implemented comprehensive public outreach campaigns that began in the pre-assessment phase (prior to submission of a Need to Change statement which begins the formal NEPA process) and continued in various public forums through release of the draft plan with alternatives. In many cases, public workshops included static maps to facilitate discussion on various topics (e.g. special places) and/or online mapping activities to gather more targeted input (e.g. opinions about designated wilderness areas) resulting in either confirmation or reassessment of draft plan components. Many of the sample forests used TPCM at later stages to present maps to the public to gauge opinions about various draft plan proposals. Table 8 provides a synopsis of public outreach and socio-spatial data collection activities 
conducted on the four sample forests that documents these activities. Additional

information can be found in the national forest briefs attached as Appendices D

(Flathead), E (Colville), F (Coconino) and G (Francis Marion).

Table 8: Tools used to collect socio-spatial data during forest plan revision by national forest sample.

\begin{tabular}{|c|c|c|c|c|}
\hline $\begin{array}{l}\text { National } \\
\text { Forest }\end{array}$ & $\begin{array}{c}\text { Public Scoping \& } \\
\text { Sense of Place }\end{array}$ & $\begin{array}{l}\text { Public Workshops } \\
\text { \& Static Mapping }\end{array}$ & $\begin{array}{c}\text { Online Interactive } \\
\text { Applications \& } \\
\text { TPCM* }\end{array}$ & $\begin{array}{c}\text { HEM** and other } \\
\text { GIS-Based } \\
\text { Applications }\end{array}$ \\
\hline $\begin{array}{l}\text { Francis } \\
\text { Marion }\end{array}$ & $\begin{array}{l}\text { Public outreach } \\
\text { (sensing) in pre- } \\
\text { assessment through } \\
\text { facilitated sense of } \\
\text { place workshops; } \\
\text { community sensing. }\end{array}$ & $\begin{array}{l}\text { Crowdbrite tool for } \\
\text { assessment reports; } \\
\text { "canvases" asked about } \\
\text { ecosystem service } \\
\text { beneficiaries and } \\
\text { special places; } \\
\text { "community } \\
\text { conversations." }\end{array}$ & $\begin{array}{l}\text { TPCM during outreach } \\
\text { on draft alternatives, } \\
\text { focusing on semi- } \\
\text { primitive recreation } \\
\text { places; developed } \\
\text { "resource integration } \\
\text { zones" for the draft } \\
\text { plan. }\end{array}$ & \\
\hline Flathead & $\begin{array}{l}\text { Public outreach } \\
\text { (sensing) for local } \\
\text { place values and use } \\
\text { preferences in pre- } \\
\text { assessment and to } \\
\text { develop Need to } \\
\text { Change. }\end{array}$ & $\begin{array}{l}\text { Community workshops } \\
\text { and place mapping on } \\
\text { topics of interest for } \\
\text { draft plan alternatives } \\
\text { development; updated } \\
\text { management areas as a } \\
\text { result of public input. }\end{array}$ & $\begin{array}{l}\text { TPCM for public input } \\
\text { into proposed } \\
\text { management and } \\
\text { wilderness areas for } \\
\text { draft plan alternatives. }\end{array}$ & \\
\hline Coconino & $\begin{array}{l}\text { Public outreach in pre- } \\
\text { assessment (sensing); } \\
\text { static maps of } \\
\text { recreation and } \\
\text { wilderness zones; } \\
\text { mailed "values" } \\
\text { written survey (during } \\
\text { early plan revision } \\
\text { efforts). }\end{array}$ & $\begin{array}{l}\text { Issues-based public } \\
\text { workshops, some using } \\
\text { static maps. Draft plan } \\
\text { submitted for public } \\
\text { review for a lengthy } \\
\text { period of time. } \\
\text { Constituent Analysis } \\
\text { on public values from } \\
\text { data collected at } \\
\text { workshops. }\end{array}$ & & $\begin{array}{l}\text { GIS-based project on } \\
\text { Coconino in } 2006 \\
\text { produced spatial data } \\
\text { layers on place values } \\
\text { and land uses. Planners } \\
\text { indicated that this data } \\
\text { only validated what } \\
\text { they already knew and } \\
\text { was not used in a } \\
\text { significant way in plan } \\
\text { preparation. }\end{array}$ \\
\hline Colville & $\begin{array}{l}\text { Outreach and public } \\
\text { sensing for sense of } \\
\text { place during pre- } \\
\text { assessment. }\end{array}$ & $\begin{array}{l}\text { Numerous public static } \\
\text { mapping activities in } \\
\text { assessment particularly } \\
\text { for recreation and } \\
\text { wilderness designation. }\end{array}$ & $\begin{array}{l}\text { Public participation } \\
\text { strategy indicates use } \\
\text { of an online interactive } \\
\text { mapping tool, but no } \\
\text { specifics are provided. }\end{array}$ & \\
\hline
\end{tabular}

*TPCM: Talking Points Collaborative Mapping tool developed through a USGS and USFS collaboration. This is an online application available to all USFS personnel that allows participants to view various spatially referenced layers, such as wilderness boundaries. It is generally used as a method to solicit public input on proposed management areas or other boundary decisions.

**HEM: Human Ecology Mapping is a GIS-based strategy in which participants indicate points, lines and polygons, usually on a static map, that are digitized into GIS layers that can be overlaid with other biophysical layers. Typically these maps are used to locate hotspots of concentrated public use or value or areas in which conflicting uses might occur.. 
Generally, values and attitude surveys occurred during the pre-assessment sensing phase as forest staff reached out to the various stakeholders and the public-at-large to inform them of the intent to revise the forest management plan. This is typically followed by workshops and other engagement opportunities, either using static mapping or an online tool (or both), during the assessment phase to inform the socio-cultural aspects of the forest assessment reports and draft plan. These workshops often focused on topical areas such as recreation preferences as well as "favorite places" activities. During compilation of the draft plan and alternatives, or after its public release, TPCM was often used to solicit public input on the boundaries of proposed special areas, such as wilderness or roadless areas, and to gauge public acceptance of the draft plan alternatives. A more sophisticated GIS-based analysis was conducted by an academic institution for the Coconino National Forest in 2006, at the start of initial public sensing and preparation of forest assessments. However, at the time the study interviews were conducted in 2016, there seemed to be limited knowledge about the data produced by this project. One participant stated that the data simply validated what staff already knew about the location of "special places" in the forest (even though its intent was to be integrated with other biophysical GIS layers to assess whether these special places were compatible with assigned management priorities, e.g. a favorite place to ride an off-road vehicle would not be compatible with protecting habit for endangered species). This suggests that knowledge of data that is not gathered and documented as part of formal assessments tend to reside in individuals. When they leave the data gets lost and forgotten. In addition, this particular GIS project was designed to produce GIS-ready data layers for integration with other ecological modelling software. Use of this kind of data would be 
indicative of the predict/model stage suggested by the Fagerholm et al. (2020) PPGIS framework, a stage in which sophisticated GIS and spatial analysis skills are required. The project did not have an accompanying "how to" manual. The social scientists interviewed had no idea how to use this data other than simply looking at the maps produced and confirming what they seemed to already know. While this project may represent a technological bridge between the social and natural sciences (from a GIS perspective), that bridge seems far from complete.

\section{THEME: Socio-spatial data influences decision-making in both direct and indirect ways.}

When asked about how socio-spatial data had been used to inform plan revision, participants were quick to offer a multitude of examples. These examples represent how the data collected impacted planning decisions in both direct and indirect ways. Examples of the integration of socio-spatial data in the forest plan revision process include:

1. Fun activity to educate the public on management issues and improve engagement.

2. Collect public input to "tell the story" that contextualizes quantitative data in the forest assessments. It was also pointed out that telling this "story" is part of the requirements of the 2012 planning rule (in sections on how the public is to be engaged in the process).

3. Stimulate conversations among interested publics - particularly in facilitating a "win-win" outcome in areas of potential use conflicts.

4. Identification of special places that informs planning decisions around what to prioritize.

5. Identification of ecosystem services beneficiaries and benefits.

6. Establishment of "communities of place."

7. Inform the Need for Change and the socio-cultural and economic Desired Conditions.

8. Gain understanding of the different uses of the forest by different communities within the forest; large forests often have very localized use patterns.

9. Reassessment of boundaries for proposed management areas.

10. Establishment of resource integration zones to address public needs. 
11. Creation of special places maps to compare with the Recreation Opportunity Spectrum.

12. Identification of use preferences to validate or revise draft plan alternatives during the public comment period.

The following text segments, drawn from the forest-level participants, provide

specific examples of these decision impacts.

There were some ideas that emerged in that process [public engagement mapping workshops] that were dramatically different than what we were thinking originally and we used that information... We completely retooled our canned set of management area allocations to better reflect front country recreation because that's what they were saying to us. We want day use opportunities that are open to us. We want our mountain bike opportunities in the front country, and we want you to recognize that, instead of putting me in the backcountry... So we really retooled based on that feedback.

So we did use that information...we spent 10 months going through public meetings where we provided very similar maps on the table. And we went through a period of 9 months with desired conditions, and we did objectives, what would you like to do in this area. We broke it down by geographic areas...so that people can relate to where they have a geographic interest so you could reach out better. And then the last one was drawing lines on the map. What should be the management areas we should be looking at? What would you like to see out here? And you know, ultimately, the goal of this was the kum-bah-yah moment when they all came together and said, "yes! If you want this and I want this then we can get a win-win!'

The geographic information was used to really look at opportunities for management in different areas...for meeting the needs and requirements under this new rule relating to ecosystem services...it manifested in the resource integration zones that the forest created in their plan. Those resource integration zones looked at those key ecosystem services that were identified and they wrote planning directions and planning components related to those key ecosystem services... That was the outcome really of that process, delineation and intent.

So one of the key findings from [TPCM] ... what they really valued about the forest is the idea that they could find solitude and get away from it all...We did ROS [recreation opportunity spectrum] mapping. When we mapped for existing, there was very little to nothing that mapped as primitive and very little area that mapped as semi-primitive...So, what they've proposed in the plan is to take some areas that are perhaps mapped as roaded-natural and transition them to semiprimitive to provide more of that experience based on what we heard from the public...And what that means is potentially closing some roads...So anyway, that's 
taking it through the process of, here's what we learned, but we had to build that into, how we were going to manage.

We had maps on the wall and we said, draw a circle around what your favorite place is and tell us what recreational activity you do in that place...I remember a moment...there was a motorized recreation group and a non-motorized recreation group looking at the maps... and so they both pretty much circled the same area, and in the conversation over why is that area so special to you, it came out that both of them enjoyed the sense of solitude that they got...So moments like that were powerful,...there was an understanding that we have motorized users that are looking for an experience that is away from roads and where they have a sense of solitude...so, we needed to think about whether we were providing anything like that...we have areas of the forest that are roaded-those are the areas that you tend to say, well, that's where we're going to put the motorized recreation. But then after those meetings and hearing that conversation about solitude, we came back and we did have discussions about-so you know, there's a segment of the motorized recreationists that we have where maybe we need to look at these roadless areas and see if we can put in some motorized recreation. So that would be trails, OHV trails, in there because there is a need for that.

The examples above refer to socio-spatial data collection in terms of enhancing public engagement and increasing understanding of what forest users value and need. In several cases the data collected resulted in revisiting and revising management options or establishing different kinds of management categories and/or boundaries. In another case, the activity itself (e.g. a special place mapping workshop) assisted in facilitating conversations and compromise around potential areas of conflict (e.g. motorized vs. nonmotorized preferences) and reassessing roadless areas to better accommodate the needs of off-road vehicle users.

In summary, the three themes described above indicate that socio-spatial data does impact forest assessments and decisions around the construction of the draft plan and alternatives. However, these impacts occur within the ID team's internal discussions and thus are not readily apparent, unless one asks specific questions. 


\section{THEME: There are significant socio-spatial data gaps around acquiring data at the local or community scale.}

When participants were asked about what they considered to be data gaps, particularly with regard to socio-spatial data, all the substantive comments exclusively revolved around the issue of scale. Participants complained about the coarseness of the social, economic and demographic data that was available to them for forest assessments, often at a county level. While they understood the importance of a regional perspective, they stated that coarse data did not fully capture the essence of the needs of local communities and were insufficient to make informed decisions about management priorities in different areas of the forest. Much of the comments indicate challenges with the Forest Planning Rule's directive to only compile "existing" data for forest assessments, which limits available data sources, to a large degree, to regional or countylevel data from government databases (e.g. U.S. Census or Department of Labor). This means that new data connected to specific places or specific local communities is not collected (though it may be anecdotally known) or incorporated in a formal way into forest plans. The following text segments provide further illustration.

There's two things going on for me around that question [data gaps]. One has to do with scale. Regardless of the content, we have very, very little information when it comes to the local community level and we rely pretty heavily on census data. We're great at a county level. But, when you're talking about a rural community that sits adjacent to an urban area, it will totally wash out what's going on in that community... being able to characterize or talk about a local community is tough for us. Especially if you're talking about existing data.

And we've wrestled with assessments. As you know, it's a collection of existing information and we have a lot of existing data on visitor use, but not a lot of existing data that is very specific to specific places on a national forest that ties to public values, benefits, preferences, those types of things. So we've had that discussion. In my mind, that's a collection of existing information that we don't have. 
And really we need to be looking at everything that's going on within that landscape at once, for NEPA efficiency, right? But yet we're not really looking at recreation or social values, in my opinion, in the right way or at the right scale...So, for example, one of the problems we have is that we have dispersed camping over everywhere. And we don't know where it is and...we need to be decommissioning roads because that's one of the things we're supposed to be doing, right? But what we're doing is outraging the public because we don't know how they use those roads...So I would want to know where they're going, what they like to do and have a really good idea about what kind of experience that they're looking for.

These text segments identify two related challenges that inhibit collection of this data - interpretation of the Planning Rule as requiring amassing existing data only for the assessments and the need to be efficient with regard to the planning process (NEPA). These represent external contingencies (legislation and administrative regulations) that place restraints on collection of socio-spatial data even when participants claim it will help in making better decisions that respond to local needs.

\subsection{Salient Themes in Coded Text Segments}

The primary goal in this study entails distilling interview transcripts into salient themes that indicate barriers, challenges and opportunities in the collection and use of socio-spatial data. Four of the themes specifically focusing on socio-spatial data have been discussed in the preceding section to establish that socio-spatial data is being collected in numerous ways and does influence planning decisions both explicitly and implicitly. Following is a detailed description of the remaining 11 themes with example text excerpts and discussion.

\section{Code: External Policy \& Law}

THEME: The PRA and OMB regulations represent a significant barrier to the collection and use of socio-spatial for research purposes, but not for on-the-ground planners who consider socio-spatial data collection as public input. 
The Paperwork Reduction Act (PRA) and the need for Office of Management and Budget (OMB) clearance for data collected from the public are clearly considered barriers to social science research and particularly affects the collection of primary sociospatial data in a research context (see Section 4.4 in Chapter 4). This is not surprising given the heavy reliance on surveys, interviewing, and focus groups within social science. This was listed as the most significant barrier by scientists interviewed from the research stations and regional offices and was strongly echoed by all scholars interviewed. One research scientist, when asked whether they encountered any legal or structural barriers in their work, emphatically replied, "Oh! Honey! Can we talk! The application, the interpretation, of the Paperwork Reduction Act as applying to research conducted in support of good forest policy and management is killing me!" This was not an isolated reaction from social scientists.

The PRA and OMB regulations represent an external contingency that the agency has little control over. This has ramifications for the use of PPGIS in collecting sociospatial data as its basic function is to collect primary data from the public (rather than using databases compiled by others, such as the U.S. Census). Efforts to secure generic OMB approval for standard USFS data collection requests are ongoing, but have not been successful thus far. As one participant noted:

In terms of the issues with OMB, I'm actually not sure, but I think that might literally take an act of Congress...Or, maybe an Executive Order would do it...no luck with that one. That wouldn't rise to the level of priority. You know, I'm not sure that there would be a reasonable expectation that those things would change. Interestingly, forest-level planners had a much different perspective. No participant at the forest-level reacted negatively when prompted about OMB approvals 
for collecting public data (cautious is perhaps a more appropriate word). While the forest-level staff certainly had a general understanding of the law and OMB regulations, as a group, forest planners and ID team members viewed the collection of socio-spatial data as public input in response to a clear mandate to fully and meaningfully engage the public in forest planning and management in a way that reflects the values that the public espouses. When soliciting information from the public, forest planners said they were careful to rely on open-ended questions and to not "tally" or "quantify" the data. As one participant explained: “...what we were told are, any specific questions that would've been asked, any tallying...we stayed away from that. What we did was pull out themes that we saw from the input."

Public Participation and Data. As the section on the spectrum of socio-spatial data documents, socio-spatial data is being collected from the public at various points in the plan revision process and in myriad ways. Although it is not explicitly stated, the study participants seem to be making a clear distinction between publicly-generated thematic data and scientifically-generated quantifiable data as well as the purpose for the different types of data (public engagement vs. forest assessment). Socio-spatial data generated through a public participatory process is exploratory and informative (as Fagerholm et al., 2020, describe in their PPGIS framework). However, technology is on a fast track. Use of the TPCM online mapping interface is being broadly encouraged, but at this point only as a public engagement scoping, or exploratory, tool. A paper describing the tool, presented at the ESRI User Conference in 2015, states that "TPCM is not a survey. It is but a public scoping tool, and therefore does not require oversight by OMB” (Aran \& Reed, 2015, p. 3, emphasis added). Furthermore, it fulfills the Forest 
Service Strategic Plan directive to "develop internet-based tools to improve internal and external user interaction with the Forest Service and Forest Service data" (USDA, 2015, p. 31, emphasis added). It is likely that as these online mapping interfaces become more cost-effective, accessible and user-friendly, their utility in gathering more expansive kinds of socio-spatial data (e.g. GIS-ready data layers) will become more apparent. This is also likely to increase incentives to be able to produce this kind of data internally. As this occurs, it will be important to understand the march of technology and have clearer definitions on what constitutes publicly-generated exploratory data as opposed to scientifically-generated data, what requires OMB approval and what does not.

1982 versus 2012 Planning Rule. Interview questions inquired about perceived differences in planning under the 1982 rule as opposed to the 2012 rule. These questions were directed at forest-level and regional office participants. Social scientists at the research stations admitted they had little knowledge of the planning rule.

Of the regional and forest-level participants who provided substantive comments, none considered the two rules as significantly different. They felt the basic premise of forest planning had not changed. As one participant noted, “...to me it's the same thing. Building a plan to go forward. And we want to make sure that we're adequately protecting the resources, trying to provide as much net public benefits as possible while doing that. In that sense, I think you can act like they're different. But, in essence that's what it's about."

However, all respondents at the forest-level appreciated the 2012 rule's new language around sustainability, ecosystem services and adaptive management (rather than the rather siloed management priority areas of the 1982 rule) and the emphasis on 
working toward "desired conditions" rather than compliance with certain standards.

They felt it provided a management structure and strategy that was much more inclusive, integrative and adaptable. Of the forest staff interviewed in forests completing the plan revision under the 1982 rule, all said that they were doing so with the intent to follow the "spirit" of the 2012 rule, which they felt more adequately reflected current planning challenges. To them, it was merely a difference in format, not function.

These findings are reported here, as the study design sought to explore differences in planning using the 1982 planning rule versus the 2012 rule. However, because participants did not expound on or reveal any strong insights or opinions regarding differences in the two planning rules, this finding is not included as a meta-theme.

\section{Code: Organizational Culture}

THEME: The culture of expertise and science (or data)-driven planning within the USFS hinders forest planning where issues should guide the process; this tends to deprioritize socio-spatial data that can shed light on these issues.

The organizational culture code did not generate an abundance of responses. Only six relevant final text segments were tagged with this code, most often in cooccurrence with other codes (science, public engagement and organizational structure). The text segments spoke of a culture of expertise, referring to the dominance of science within USFS culture and practice. Participants mentioned how this culture of expertise resulted in a planning process that tended to focus on finding data rather than on broader management issues and tended to result in deprioritizing (but not expressly devaluing) the collection of socio-spatial data, primarily due to challenges related to staff time, budgets and available skill-sets. These types of comments were not ubiquitous within this dataset, but did represent quite strong opinions about this topic. The following excerpts 
express this sentiment around the challenge that a science-driven planning culture

presents for forest planning:

...issues tell you what the data needs are that you have to deal with...if you try and do all uses, on all forests, and all possible interactions, and all possible landscape characteristics, and all possible political-economic implications, you can't do it! .... there is a] data-driven science-oriented culture of the Forest Service...So, if we revise planning to be more of a strategic planning, collaborative activity, then that larger scale, multi-forest approach could really be operationalized. But the first thing we're going to do is collect all the science that we've got related to all issues and all topics in the science synthesis...that's getting into the weeds.

...to me that comes to the point where data becomes less important than just putting some basic rules into place about how you go about planning and then adapting as you go. That's a hard thing for this agency to deal with... because we're very much scientists and we want to have everything perfected on that front. ...We've got to be thinking more about how to be adaptive... The public gets it. It's internally - the agency specialists - that struggle with it. I say, we're gonna get stuff wrong...And when I tell the public, they're okay with that...But we get paralyzed by this stuff... They [the resource staff] want to make sure that they've got that plan component tee'd up correctly. And, you know, it's okay if you don't! It's hard for them to do that...because they're so concerned about some resource not being protected adequately.

Notes on the "Science-Driven Culture." There is consensus among study participants that the USFS is a science-driven agency. When asked about the culture of the agency, participants immediately emphasized excellence in applying cutting edge science and science-driven decision-making. From an organizational culture perspective, this would be considered the formal agency "who we are" statement. While participants were open in discussing the challenges around the culture of expertise (e.g. data obsession), what is perhaps more interesting, and what the interview prompts did not dive into, are the beliefs, attitudes and values that lie below the surface.

For example, the two excerpts above were drawn from comments made by natural scientists and seem to refer to the intense analyses and modeling that occur in the 
biophysical sciences, in areas such as estimating fuel loads for wildfire management or vegetation assessment for habitat protection, that make up a large portion of the forest assessment. What social scientists have to say about a culture of expertise is more nuanced and typically embedded in comments made around organizational operating protocols and staff capacity (which will be highlighted and discussed in the following section). These types of cultural references are what Schein (2010) refers to as the "cultural DNA" of an organization. They are the deeply embedded, often unconscious beliefs and values that are not "public," but instead manifest in the organization's structures and processes. From the perspective of embedded culture, two important questions remain that are open to supposition, as interview prompts did not specifically address them: (1) what "science" defines the USFS as a science-driven agency? and (2) if socio-spatial data is considered public input, and not science, how does that affect decisions around allocation of staff time and resources? These questions overlap with many other themes, particularly organizational structure.

\section{Code: Organizational Structure}

The Organizational Structure code was used to highlight text that talked about standard operating procedures, roles and responsibilities, communication protocols, decision processes, and incentive structures within the agency. This code represents the formalized operations of an organization. This is also the code with the largest number of text segments $(\mathrm{n}=75)$. The synopsis below provides typical comments relating to themes around social science research (staff incentives), data access and dissemination (constrained by staff capacity), and planning strategies for forest social assessments (standard operating procedures). As well as being themes themselves, the text segments 
included here also highlight numerous linkages with other codes, particularly

organizational culture as described above. The three organizational structure themes will be presented with example text segments followed by a discussion.

\section{THEME: Promotion incentives for social scientists at the research stations focus on publishing in peer-reviewed academic journals rather than formats which may be of more use to planners.}

Staff incentives within the USFS research stations create challenges in disseminating useful social science research and results to on-the-ground land managers. It should be kept in mind that the mission of the research and development arm of the USFS is to conduct cutting edge research to address future forest management challenges, not necessarily to inform current issues (refer to Section 4.3 in Chapter 4 for a more detailed description of the mission of the research stations). The following excerpts explain further:

I think the National Forest System is sometimes a little confused about what research is as opposed to what staff work is. So they think that research ought to answer their technical questions, quick, quick...and then research will push back and say, hey, no, this isn't really a science question so it's not under our legislative authority or mandate...Just a difficulty in understanding what different people's roles are...scientists write papers, planners write reports.

I think one of the challenges you face when you're a scientist for the research stations is that you're expected to contribute to the scholarly literature. You're expected to publish peer-reviewed journal articles for the science community. Those often aren't good products for the managers making decisions. You're also expected to deliver information in a way that's useable by the managers on the ground. Often that could take the form of a general technical report or some sort of a white paper, briefing paper, what not. I always, often, feel like I have to double publish my research results to meet these different sorts of audience needs. It's really demanding because I feel like I end up doing one or the other...It's pretty frustrating in that regard.

Too few social scientists. The scarcity of social scientists within the agency

clearly create incongruence between the function of the research scientist (conduct 
research and publish) and the function of program managers (gather relevant scientific information to make on-the-ground decisions). Because there are so few, social scientists at the research stations are often put in a position of choosing to serve one or the other functions. This is a typical trade-off scenario. The question becomes, what are the consequences? Does cutting edge research suffer? Does lack of access to current research findings hinder a forest planner's ability to make well-informed decisions?

\section{THEME: Data access and a social science support portal are needed to more effectively transmit knowledge about the collection and use of socio-spatial data in planning; challenges are directly correlated with staff capacity.}

This theme is directly related to the theme above, which asserts that incentives for social scientists at the research stations focus on publications in peer-reviewed journals. This is a format that is not all that useful for planners and results in challenges with disseminating cutting edge research findings to on-the-ground staff. Staff capacity issues, particularly within the social sciences, have highlighted a need to prioritize creating a data dissemination portal and social science support site as the following excerpts suggest:

It's not always easy to come up with tools and I feel like the agency struggles because every forest tries to invent their own, whether it be an interactive map or a way to get the public to be engaged... how do we share ideas, tools, ways of doing things - what didn't work, what did work - even as mundane as records and data management because so much is produced. It's hard to keep track of the file management. I think information sharing is really important...We're all so busy in our own little worlds that it's hard sometimes to pick your head up and investigate what others are doing. But that would be in terms of how to make the process better...I don't know how to do it.

... when we [social scientists] all get together at a meeting and talk about it [how to incorporate public values into planning], it [data access portal] seems like a good idea but as soon as you disperse back into your work that you need to do, you're instantly swallowed up in everything that needs to be done immediately and it's hard to carve time back out to do anything extra. There's so few of us; it's 
hard to get the capacity to do the extras to get momentum moving or to advocate for other stuff."

Participants felt that creating a centralized portal would provide a means to enhance organizational learning and adaptability around social science and use of sociospatial data. Social scientists, especially in the regional offices, have tried to create a professional community of practice, but time pressure, in particular, has made that difficult. A data portal would more efficiently and effectively communicate and disseminate social science research, methods and tools. It can also help to demonstrate the utility of integrating socio-spatial data into standard operating processes, particularly what it is, how it has been or should be integrated into planning.

\section{THEME: Socio-spatial data belongs in the public sensing phase of planning to better engage the public as it would contribute data for the development of the Need for Change and Desired Conditions statements; challenges are directly related to staff capacity.}

Many participants (at both the regional and forest level) talked about the need to rethink the plan revision strategy and process and do social assessments early, including much more data collection around sense of place, special places and other types of public land-use preference information. Comments reflect more of a wish list and fall under two generalized factors: (a) having sufficient data about public values and preferences to inform the initial Need for Change and Desired Conditions statements, and (b) acknowledgement and validation of the value that socio-cultural and socio-spatial data bring to the table in the planning process. Participants offered the following suggestions:

So if I could, if I had my way, I would start before the budget clock started [the formal NEPA process] and do pre-assessment work...And I think the assessment for social would have the most impact. Then that information does roll into the need for change and right into the rest of the document [draft plan] and into the EIS. I think if you don't start that out well, with your need for change, then 
you're never going to get to the correct need for change. And then you're going to struggle for the rest of the process.

[G]et your inventories done either before or during the assessment phase...It's very important to be prepared when you're in [ID] team discussions, to have all your maps on the table. If you're there as a social scientist, and you're really not prepped with your inventories and your maps, then it's easy for everyone else not to see the importance of that component. You don't want to have all the biologists just going and doing their thing like you're an afterthought...And, I would definitely do some of that community sensing and community engagement really early...Yeah, sense of place, all that; it's just important to get that done early.

Social scientists and planners working on-the-ground understand that socio-spatial data play a key role in successfully framing the initial stages of a planning process in line with what the public values. This is consistent with the discussion in the introduction around the importance of understanding human-environment connections to increase public engagement and decrease potential conflicts as the planning process moves through its stages. However, looking at these three themes, there are factors identified by participants that make this goal challenging, two that are expressly stated and a third that is implied: (1) limited staff capacity or resources to do comprehensive data collection upfront; (2) need for a community of practice to disseminate best practices and (3) expectations about what a social science needs to bring to the table in order to be noticed. This begs the question on the extent to which socio-spatial data (and, if I may be so bold, social science in general) is being (de)prioritized within the planning process (this also harks back to the question, what constitutes science in a science-driven agency).

The dataset does not delve into these questions in enough detail to make definitive conclusions, but if this is indeed the case, what is the impact on the quality of the sociocultural assessments that are used to make planning decisions? Is there a trade-off occurring here, where one function (biophysical modeling) is being prioritized over 
another (public values)? As Rose, et al. (2020) note, capacity issues tends to direct focus on fulfilling the public participation requirement in accordance with regulations and neglects the quality and effectiveness that the participation process could provide. Is the kind of public collaboration and consensus building, that activities involving collection of values-based data upfront can engender in forest management, being overlooked?

\section{Code: Organizational Leadership}

\section{THEME: Organizational leadership acts as an enabling factor; supportive leadership results in effective collection and integration of socio-spatial data.}

The Leadership code occurred most often in conjunction with various other codes and seems to be an enabling theme that creates positive links between decisions that leaders make and successful planning strategies. For example, a forest planner may be speaking about how socio-spatial data was collected during a public workshop, adding that the success of the effort was the direct result of strong support from the forest supervisor as well as allocation of sufficient resources in which to conduct these activities. Within this category, 16 text segments fell neatly into two groupings -Leadership Support (or lack thereof) and Decision-Making. Positive comments revolved around appreciation for strong leader support of the ID team and the planning process and how that directly translated into effective and efficient strategizing, communication, trouble-shooting, and decision-making during the long and arduous process. These comments were consistent among participants at the forest level. Challenges in leadership (often worded as a lack of leadership) were mentioned only at the regional and research station levels and included lack of leadership will to prioritize social science in 
general (research stations) and insufficient measures to adequately address a persistent overwhelming workload among social science staff (regional offices).

Leadership is an important theme generally, especially as leaders make the decisions on what structures to put into place for the planning process, how available resources should be allocated, etc. Participants in this study did not care to elaborate extensively when prompted about leadership, except to connect it to other factors. It is included here as an explanatory theme that begs further research.

\section{Code: Organizational Budget}

\section{THEME: Organizational budget reduction is directly related to staff capacity and planning strategy resulting in a lack of an ability to collect socio-spatial data.}

As might be expected, there were no positive comments about the budget at any of the functional levels. It is well known that the USFS budget has been shrinking for many years. As in the case of the Leadership code, the Budget code seems to serve as a strong explanatory (and might be argued, a causal) factor that directly contributes to barriers in the collection and use of socio-spatial data. As is to be expected, the Budget code was highly correlated with the Capacity code, particularly as it relates to inadequate staffing and expertise to collect socio-spatial data for forest planning. Budget reductions is the most common barrier mentioned by participants. The following text excerpts illustrate representative comments about the effects of a scarcity of staff with social science backgrounds and the necessity to "pick and choose" what social assessments or data collection can be done with limited staff and financial resources. The text segments also illustrate connections with numerous other factors, such as standard operating 
procedures (planning protocols), decision-making, leadership, and prioritization of planning tasks based on legal considerations.

Ifeel like it [the budget] probably is getting worse... a lot of times you don't hire positions behind people who leave... there's the fear of are we going to have the budget to continue to pay that person year after year?...You know, you have the big idea, there's one roadblock or bump, and then it just gets thrown out because we don't have the capacity to make it happen long-term, so we're going to have to go for some lower-hanging fruit.

The funding mechanisms are very tight. You've got to pick and choose your battles. So I said, you know what? We're going to have to figure a way to look at the social information that we do have and make that be the basis for our analysis...you've got to make choices.

So when budgets get cut...you know, they have certain legal requirements that they have to comply with... They don't really HAVE to do the social science from a legal standpoint. I mean...it's like, you know, you HAVE to comply with the ESA [Endangered Species Act] requirements.

An intractable budget or budget choices? Comments from study participants

indicate that they feel the budget is an intractable, external contingency that they have little control over. This is important to point out as it indicates an attitude resigned to the fact that they must figure out how to do more with less and thus it is a rational choice to prioritize what is legally required (e.g. ESA compliance) as opposed to what is only suggested, but not required (e.g. collecting socio-spatial data). All forests are under significant public and political pressure to conduct their business in an efficient and costeffective manner. Again, trade-offs are inevitable. However, there is a level of choice here that harks back to the discussion in the themes listed in organizational culture and structure. Yes, there is a finite amount of money to work with, but there is also some latitude in how that money is allocated. Deliberate choices are made to go for the "low hanging fruit" in one area rather than another. 
It is not the purpose of this study to make judgements on whether these choices are correct, only to present a compelling observation that budgetary matters may not be as "intractable" as participants perceive. The processes put in place often reflect what is deemed a priority in producing an outcome. As is the case for any trade-off (and something that would be interesting to explore) are the consequences of those trade-offs.

\section{Code: Organizational Capacity}

\section{THEME: The scarcity of staff in the regional office and at the forests with experience and skills in social science and/or PPGIS limits the capacity to collect socio-spatial data; directly correlated with Budget.}

Organizational capacity garnered a significant number of text segments and a high rate of co-occurrence with the Budget theme. Two related factors are evident in this theme - the negative effects of staff time pressure and, to a lesser extent, turnover rates. This topic was of greatest concern to forest- and regional-level staff. Of the 34 (often extensive) text segments making up this theme, only two saw reasons to be optimistic about capacity issues. One appreciated the "energy and ideas" that new staff members bring to the ID team as a result of staff turnover and the other recognized that the broader monitoring mandates in the 2012 planning rule might propel the agency to invest in staff capacity-building. While these statements are interesting, they are also a valiant attempt to answer what was probably an overly leading prompting question.

Among forest and regional office staff, the challenges with regard to staff capacity most definitely outweigh any advantages. Some effects of the lack of capacity include: (1) inability to collect new or more relevant socio-spatial (and other kinds of socio-cultural) data; (2) use of temporary contractors to supplement needed expertise that does not build internal staff capacity; and (3) no time to discuss lessons learned with 
professional colleagues or organize socio-cultural or socio-spatial data for more

widespread dissemination. Representative text segments follow:

So we've become, compared to what we were, a very lean organization...We haven't staffed up in any of the areas where we should probably be putting more emphasis, like recreation and more of the social side of our business. We've kind of cut ourselves down to the quick and haven't really recovered. There are capacity issues that are huge. So when the regional foresters say, yea...you're going to do this [plan revision] in four years, don't worry, we'll throw money at you. We think, well, yea, you can throw all the money at us you want, but we don't have the capacity. We don't have the skills in place either. That's a barrier.

Yup, and it's an organizational thing too, you know. It's the way that the agency has allocated capacity at different levels and different areas...so that when the forest needs it [temporary contractors], it's available. But the sort of fleeting or temporary nature of it...it allows them to get that project done, but then how do you maintain that, and especially when there are personal relationships involved and how important those are to actually carrying out work on the ground? I see those as barriers, but probably realities more than anything.

Okay...in retrospect, I think that probably forging ahead was the biggest thing on our minds. We just had to move forward, whether we had data or not. And again, there's a lot more support on the biological and physical side of inventory and monitoring and all this other stuff that feeds these massive data sets. It's not always the same for social stuff. So I would have had to identify early that I want community maps of whatever, this and that, and community benefits, sense of place maps, and all these things. I think I would have really had to get on that early. And plus there was sometimes just a lack of staff time when you're having to do all the other inventories at a minimum. You've got to do ROS, wilderness, scenery, rivers. And then I have to figure out whatever other stuff I might need. So I would say I didn't identify them early enough, but there's the capacity issue.

A related, but less pervasive, factor that participants mentioned around staff capacity involves staff turnover, whether it be retirements, transfers or an employee that leaves and is not replaced. Comments on the effects of turnover, particularly as it relates to the plan revision process, include: (1) significant delays and disruptions in the process as new team members get up to speed; and (2) loss of institutional knowledge. 
I would say that a challenging area in working with the regional office over time, and it's for everywhere, it's for any forest or region or district, is just the turnover of leadership and kind of on-the-ground capacity you lose when you do have turnover...you're surging ahead under one model or one kind of a working model of how you communicate and how you share information and how you get the job done, essentially, and how reviews are done. And then you have several positions turn over and there's the forming, learning, performing, redesigning the whole diagram. And sometimes some of those are good, and some of those aren't so good. And I would have to say that from my experience it seems like at a certain point in time...there were so many positions that turned over, we just really lost steam.

Participants, particularly from the regional offices, indicate a strong desire to explore and engage in collecting socio-spatial data to better reveal public values toward the forests. However, the there are few social scientists in the regional offices (and even more so at the forest level) make that desire seem almost impossible to realize. Among participants, there was both resigned acknowledgement that there are limited amount of resources to work with and also frustration. One participant went so far to suggest that each open position in the USFS should be filled by a social scientist. Participants want to be able to do their job well, to collect more data, to be more responsive to public values, but this is made much more difficult by an already over-filled plate of required analyses and tasks.

\section{Code: Research \& BASI}

\section{THEME: Socio-spatial data retrieved from non-traditional sources would help to address a data gap in acquiring more localized community-focused datasets to inform planning.}

This code did not generate a large amount of text segments relevant to a discussion about factors in the use of socio-spatial data $(\mathrm{n}=11)$. The prompting question may have been too specific - asking how BASI was defined in the assessment phase of plan revision - with insufficient follow-up questions. In addition to simply providing a 
canned definition of BASI, some participants did provide some unique insight and ideas. A few challenges mentioned include a perceived reluctance by forest and regional scientists to turn to non-traditional or external data sources (such as datasets compiled by other government agencies, special interest groups or through citizen science) as valid BASI and a reliance on coarse, as opposed to localized datasets, particularly in the sociocultural and economic assessments. These are both related to a science-driven culture and organizational capacity. Both of these themes impact the procurement of sociospatial data for forest assessment and monitoring purposes. For example, if securing OMB approval represents a significant barrier in the collection of public data (either in research or planning), datasets of public landscape values and use preferences gathered by other sources (often at a much more localized scale) may provide an alternative, yet apparently ignored, source of information.

\section{Code: Science Networking}

\section{THEME: Interdisciplinary work is valued within the agency, but expertise siloing and language barriers hinder a better understanding of the value of socio-spatial data in informing land-use decisions.}

The Networking code was used to tag responses that spoke to how scientists interacted or partnered with each other (intra- and inter-agency as well as between natural and social scientists) and under what circumstances or contexts. Participants at the forest level, in particular, spoke at length in response to prompts on how they worked with other scientists within and external to the agency. Strategies for and experience with sharing expertise and forming interdisciplinary relationships dominated the text. The tone of the text is predominantly positive, although many were quick to point out persistent challenges. 
Opportunities. Participants note that new language in the 2012 planning rule and directives have created welcome opportunities for increased interdisciplinary networking among scientists within the USFS and with external partners. Many feel that the rule's emphasis on sustainability, ecosystem services and adaptive management provide a more integrative structure that incentivizes interdisciplinary work. This was particularly appreciated by the social scientists on ID teams. As one participant noted:

I think sustainability can be achieved - and it's all about values - how much of one versus the other. So there were a lot of conversations about what could be done...Yeah, and that was one of the really fulfilling aspects of it. We had internal engagement and then pretty good external engagement as well. I feel like it's different from our normal NEPA projects in that we're looking to the future...It allowed a little more space to talk and not put us in an adversarial role. I had really good team members who could engage in those discussions without wondering about how to facilitate that conversation. How do we find a good balance point?...I felt like we were at least able to articulate what the interests are on all sides, on all sides, I guess.

Challenges. Participants conveyed general optimism and professional satisfaction around interdisciplinary work within the agency (there are a few negative stories told by scientists at the research stations, but in reference to relationships with other scientists at academic institutions, not within the agency). However, many participants noted that there are still persistent challenges. The majority of these challenges revolve around expertise siloing exacerbated by the difficulties in developing a common language that is needed for interdisciplinary and integrative research and planning. It is important to note that the comments around siloing refer to areas of scientific expertise, not siloing around the agency's functional operations. The following text excerpts highlight participant thoughts on these challenges:

Well, it's very telling when I sit in a forest planning team meeting and there's nine physical or biological scientists and one social person...Like, oh it's all about 
ecological integrity. Well, yeah, but the reason we are doing that is because...people have a value attached to that...So it's very interesting that the conversations don't go there very often. It just seems like sometimes I'm just sitting there thinking, well, okay. I'm just telling you, there are still communities out there, there are still people...But I think that's one of the barriers. There's sometimes the...language, the common language, sometimes isn't there.

Well, I think the only tension is first that every expertise, every group has their own language. And that's one thing that when I first started working with some social scientists, I felt like I was teaching them a whole new language and they're teaching me a whole new language. So, in some ways it's just a matter of how you've been trained in a large system, how you perceive the world and to express your understanding of it. And, if you've been trained in really different ways then sometimes people can't really see the overlap.

\section{A structure that supports interdisciplinary work can be leveraged to advance} social science and the collection of socio-spatial data. While I have posed questions that suggest organizational culture, structures and processes within the planning system may result in deprioritizing social science and socio-spatial data, this does not detract from opportunities presented by the apparent eagerness on the part of USFS social scientists to engage in interdisciplinary work, despite the challenges. This is one aspect of a science-driven culture and institutionalization of interdisciplinary processes (via ID teams) that offers opportunities to advocate for and advance the collection and use of socio-spatial data. Bennet et al. (2016), for example, suggest mainstreaming the social sciences within natural resource management by: (1) including social scientists at all stages of planning and implementation; (2) building the capacity of social science within agencies; and (3) active involvement of social scientists in policy-making. These suggestions do put the burden of advocating for social science and socio-spatial data on the shoulders of the few social scientists within the agency. But, as the saying goes, where there is will, there is a way. 


\section{Code: Public Engagement}

\section{THEME: Public engagement is a necessary and on-going activity in forest planning with numerous opportunities to collect data on public values and preferences beyond forest planning.}

Public engagement was a popular topic of discussion for forest-level and regional participants (scientists at the research stations generally are not involved in public engagement within a forest planning context). Out of the full dataset under this code, about $25 \%$ of the text segments directly related to activities around data collection, as opposed to a more general discussion of public participation strategies in general. In that sense, these text segments contain significant insight and represent compelling opportunity factors in the collection and use of socio-spatial data in the plan revision process.

Forests utilize numerous strategies to outreach to the public and other interest groups including open houses, workshops (with or without mapping activities), information sessions, sensing calls, webinars, field trips, web-based applications and commenting tools, and special interest or collaborative meetings. Participants recognized the critical importance of public engagement, but made no claim that this process was easy. On the contrary, public engagement is difficult and study participants were forthcoming in discussing some of the challenges they faced during the public sensing, scoping and commenting process. Challenges that seemed to be common include: (1) facilitating and reconciling conversations among polarized public positions; (2) low public turnout at events; and (3) difficulty in reaching a diversity of stakeholders or worrying that only the loudest or more organized voices are heard. 
Study participants, primarily members of the ID planning teams, also recognized opportunities. They used numerous public engagement strategies and tools to collect public input to supplement coarser socio-cultural and economic data from academic sources or government databases. They also valued the opportunities that forest plan revision presented such as: (1) educating the public on forest management challenges; and (2) stimulating conversations to reach consensus about management priorities.

The text segments below provide a few representative samples that most certainly speak to challenges, but also provide a hint of the opportunities that public engagement represents.

We traditionally have just gone about that in the NEPA way of public input. You know, scoping, notices...So we're trying to develop a strategy for how we can, not just communicate...it really is to get a sense of how it affects communities. To what extent perhaps could we partner, work together, towards kind of achieving a common goal, when that can be achieved?... So we're exploring that and working with public affairs on that and literally putting together kind of a strategy to help forests to get that understanding of what is really valued because we have a hard time sometimes knowing.

I think generally it [public engagement] was trying to confirm what we, the specialists, and employees intuitively and anecdotally and sort of just know by working on the forests, what they knew. So, how do you confirm that in a concrete fashion? We relied on existing data and at the same time I felt like, how much of that could really provide us good information? That's where I think we did our best to supplement with public meetings and public input where we didn't have hard data.

And I talked to them for 10 months straight -- tell us what you want from your government...this is exactly what you want your government doing, asking the public what they want instead of taking information then we go in our black box and we do something with it. This is your [the public's] opportunity to come to agreement. There was polarization on the issues; we couldn't get to full agreement. But we did get good feedback, even with the people that were so critical.

I think one of the dangers of so much forest management now and the emphasis on the collaborative process - I mean we have all these forest collaborative 
groups...those people are really engaged in that process and they have a big influence on it...They're getting all this input from all these very engaged stakeholders, but they're not getting input from the silent majority. So I think by doing social science research to see what that larger public cares about, it's a good thing. How will that get incorporated into decision-making? I don't know, especially if it's different from the people who show up at the collaborative meeting every month.

Collecting socio-spatial data requires public participation. Discussions about the barriers and challenges in collecting socio-spatial data cannot occur without also talking about public engagement. The two processes are inextricably linked. Sociospatial data requires public input. In this sense, public engagement as a means to expand the collection of socio-spatial data represents an opportunity that can enhance, and extend beyond, forest plan revision. Participants were eager to talk about ideas and suggestions toward this end. Potential opportunities and participant suggestions for expanding the socio-spatial data toolkit will be discussed further in Chapter 8 .

\subsection{Summarizing the Themes}

The exploratory design of this study sought to wade through numerous participant comments to better understand the how forest planners are collecting and using sociospatial data and to determine the most salient factors that impact these activities. The findings documented above present these factors as thematic statements. These themes are summarized in Table 9 below, with their associated keywords (that will be used in the socio-spatial thematic network described in the section that follows). Following the table is a brief discussion of functional level differences, noting how some themes have different impacts on participants at the regional office and research station levels. 
Table 9: Summary of themes in the collection and use of socio-spatial data by thematic statement and keyword.

\begin{tabular}{|c|c|}
\hline Thematic Statement & Keyword(s) \\
\hline $\begin{array}{l}\text { Socio-spatial data collection tools take many forms and produce different kinds of } \\
\text { data. } \\
\text { Socio-spatial data collection tools are employed at different points in forest planning } \\
\text { and for different purposes. } \\
\text { Socio-spatial data influences decision-making in both direct and indirect ways. }\end{array}$ & $\begin{array}{l}\text { Tools } \\
\text { Data Formats } \\
\text { Decision } \\
\text { Impacts }\end{array}$ \\
\hline $\begin{array}{l}\text { Socio-spatial data belongs in the public sensing phase of planning to better engage the } \\
\text { public and contribute data for Need for Change and Desired Conditions statements. }\end{array}$ & $\begin{array}{l}\text { Planning } \\
\text { Strategies }\end{array}$ \\
\hline $\begin{array}{l}\text { The PRA and OMB regulations represent a significant barrier to the collection of } \\
\text { socio-spatial for research purposes, but not for on-the-ground planners who consider } \\
\text { socio-spatial data collection as public input. }\end{array}$ & $\begin{array}{l}\text { Socio-Spatial } \\
\text { Data is Public } \\
\text { Input }\end{array}$ \\
\hline $\begin{array}{l}\text { Public engagement is a necessary and on-going activity in forest planning with } \\
\text { numerous opportunities to collect data on public values and preferences beyond forest } \\
\text { planning. }\end{array}$ & $\begin{array}{l}\text { On-Going Public } \\
\text { Engagement }\end{array}$ \\
\hline $\begin{array}{l}\text { The culture of expertise and science (data)-driven planning within the USFS hinders } \\
\text { forest planning where issues should guide the process; this tends to deprioritize socio- } \\
\text { spatial data that can shed light on these issues. }\end{array}$ & $\begin{array}{l}\text { Science-Driven } \\
\text { Planning }\end{array}$ \\
\hline $\begin{array}{l}\text { Interdisciplinary work is valued within the agency, but expertise siloing and language } \\
\text { barriers hinder a better understanding of the value of socio-spatial data in informing } \\
\text { land-use decisions. }\end{array}$ & $\begin{array}{l}\text { Interdisciplinary } \\
\text { Work }\end{array}$ \\
\hline $\begin{array}{l}\text { There are significant socio-spatial data gaps around acquiring data at the local or } \\
\text { community scale. } \\
\text { Socio-spatial data retrieved from non-traditional sources would help to address a data } \\
\text { gap in acquiring more localized community-focused datasets to inform planning. }\end{array}$ & Data Gaps \\
\hline $\begin{array}{l}\text { Data access and a social science support portal are needed to more effectively } \\
\text { transmit knowledge about the value of socio-spatial data in planning. } \\
\text { [Related to the theme which states promotion incentives for social scientists at the } \\
\text { research stations prioritize publishing in peer-reviewed academic journals rather than } \\
\text { formats which may be of more use to planners.] }\end{array}$ & Data Portal \\
\hline $\begin{array}{l}\text { The scarcity of staff in the regional office and at the forests with experience and skills } \\
\text { in social science and/or PPGIS limits the capacity to collect socio-spatial data. }\end{array}$ & $\begin{array}{l}\text { Lack of Staff } \\
\text { Capacity }\end{array}$ \\
\hline $\begin{array}{l}\text { Organizational budget reduction is directly related to staff capacity and planning } \\
\text { strategy resulting in a lack of an ability to collect socio-spatial data. }\end{array}$ & $\begin{array}{l}\text { Budget } \\
\text { Reductions }\end{array}$ \\
\hline $\begin{array}{l}\text { Organizational leadership acts as an enabling factor; supportive leadership results in } \\
\text { effective collection and integration of socio-spatial data. }\end{array}$ & Leadership \\
\hline
\end{tabular}


Themes and Functional Level Differences. The themes in Table 9 reflect consistency of participant responses in all the administrative units included in this study (research stations, regional office and forest-level). There are only a few differences noted in this dataset between these units, not in the salience of the theme, but how it impacts a particular cohort of participants. For example, OMB approval is a theme that crosses functional boundaries. The social scientists at the research stations, however, consider OMB regulations to be the most significant obstacle as it severely hinders their ability to collect data needed to conduct their research. Participants from the regional offices and forests view socio-spatial data within the lens of public participation; it is the output of public engagement strategies. In addition, challenges in the dissemination of and access to research, data and other crucial information is a consistent theme, but impacts scientists at the research stations differently than regional and forest-level staff. Research station scientists talk about incentives that prioritize journal publications over the technical reports that planners prefer, while regional and forest-level participants talk about lack of a social science data portal and time to read and absorb all the latest scientific research. And, finally, the scarcity of social scientists within the agency affects staff capacity at all functional levels, but most significantly for participants at the regional offices. The social scientists within this cohort experience an often overwhelming workload, particularly with regard to supporting the data needs of the forests.

\subsection{The Socio-Spatial Thematic Network}

The final analytical tool applied in this study explores and visualizes linkages between the themes by constructing a thematic network. The thematic network technique is a structured methodology for the systematization and presentation of qualitative 
analyses. It produces web-like illustrations that identify system elements and show linkages between them (Attride-Stirling, 2001). The specific steps to create a thematic network have been explained in Chapter 6, Section 6.6. Appendix K (Thematic Network Organizing Criteria) provides detail on the specific criteria applied to organize and link themes derived from this study's dataset into the socio-spatial thematic network displayed below. As an interpretive tool, the thematic network serves as a revised conceptual framework that addresses the study's objectives.

Figure 8: The socio-spatial data in forest planning thematic network.

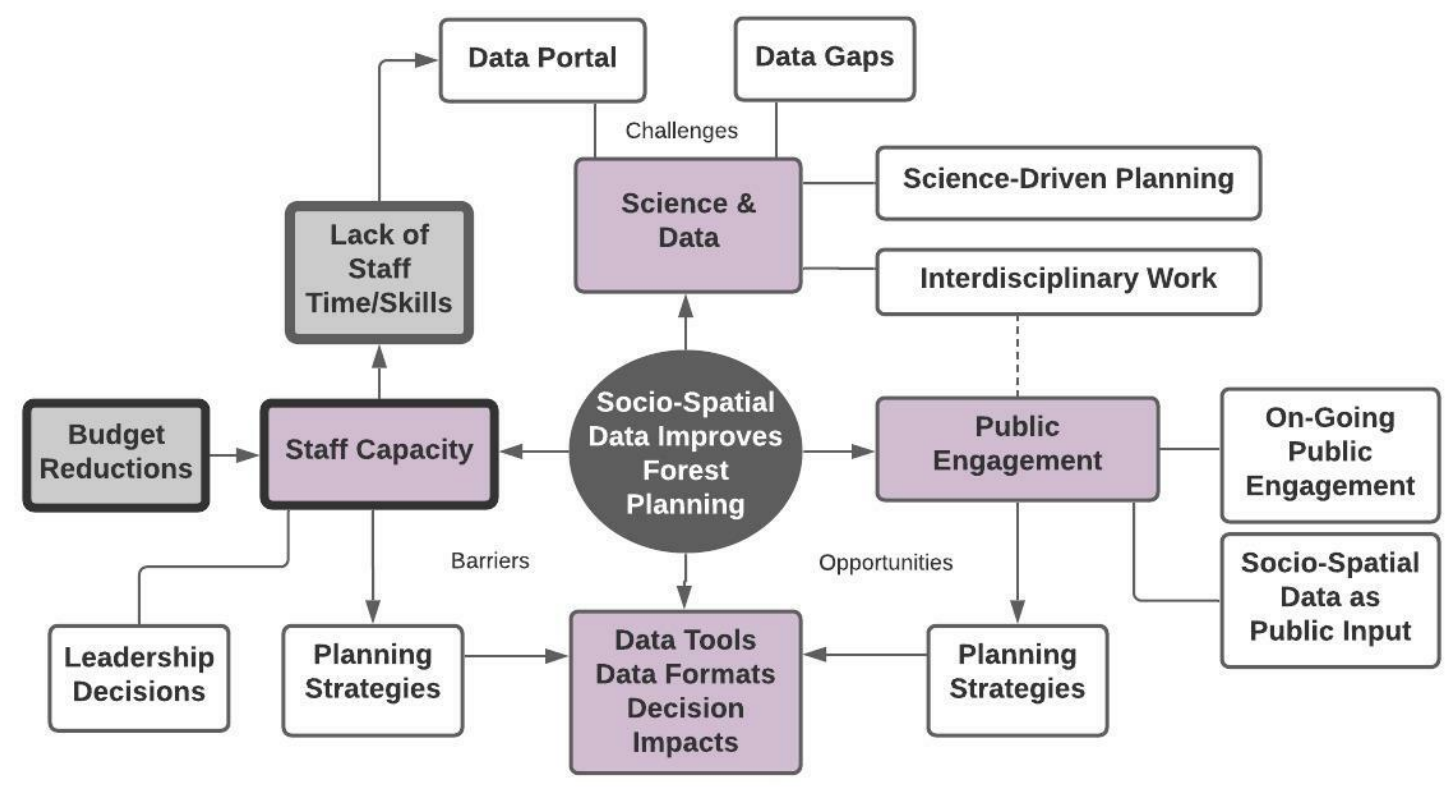

Interpreting the Socio-Spatial Thematic Network. The socio-spatial thematic network provides a visualization of a system linking themes into sets that represent barriers, challenges and opportunities around the collection and use of socio-spatial data. The visualization uses a simple mind map graphic that is a popular tool in systems science to organize ideas and concepts (see Buzon, T., 2018, Mind Map Mastery). Mind maps build an intuitive framework around components linked to a central concept. The socio-spatial data thematic network is not intended to display the entire forest planning 
system, just those elements study participants indicated were significant with regard to socio-spatial data.

The Global Theme - that socio-spatial data is deemed valuable in forest planning - is a unifying concept consistently expressed by all study participants. The global theme links to three organizing themes (Science \& Data, Public Engagement, and Staff Capacity). Attached to each organizing theme are their associated basic themes derived from the thematic analysis, displayed as keywords (refer to Table 9 on page 127 for the associated thematic statement). The Data Tools, Data Format and Decision Impacts themes are extracted as a separate thematic category. They represent unique inputs and outputs of the forest planning system and are connected to all the organizing themes through Planning Strategies that represent decisions made on when, where and how to collect socio-spatial data within the planning process. The three organizing themes represent opportunities, challenges and barriers:

1. Public Engagement: This is the organizing theme representing socio-spatial data's highest "value add" within the planning process with basic themes that identify possible leveraging opportunities.

2. Science \& Data: These are themes that represent critical processes and inputs into the system related to data and data dissemination that participants often described as challenges, but not necessarily insurmountable.

3. Staff Capacity: This is the most significant barrier identified by participants around effective collection and use of socio-spatial data, strongly linked to Budget issues, which participants perceive to be an external and intractable contingency. 


\subsection{Identifying Barriers, Challenges and Opportunities}

Barriers. Significant barriers identified by study participants include themes associated with staff capacity. Arrows indicate a system flow or causal path. For example, budget reductions lead to insufficient staffing which affects staff capacity which results in a lack of staff time to collect and/or use socio-spatial data. Residual flows occur around role of leadership, particularly as it relates to decisions regarding what planning strategies will be employed or where resources will be allocated - will sociospatial data be collected or not? The lack of staff capacity also connects to the Science \& Data thematic group. Lack of capacity creates significant challenges in staff ability to create or maintain a data portal for dissemination and networking purposes or address identified gaps in socio-spatial datasets through additional data collection activities.

Challenges. Themes that are associated with Science \& Data are considered persistent challenges, but not necessarily impossible to address. These include comments by participants around data gaps and the desire to create a data portal for better networking and sharing of best practices. These themes are impacted by staff capacity issues; participants noted they simply lacked the time to create and maintain a data portal or add extra tasks to their already full plates to gather (or even advocate for) socio-spatial data outside of what was expected. This thematic group also includes comments about challenges around interdisciplinary work. Interdisciplinary work and the focus on science-driven planning form a loop. For example, participants spoke about challenges around developing the language and culture for natural and social scientists to work better together. Participant comments subtly suggest that social science is not as valued as biophysical science and leads to a tendency to prioritize quantitative data that is 
considered BASI and not fully realize the potential of qualitative socio-spatial data to inform planning decisions and improve public engagement. This may result in a lack of cross-disciplinary understanding about what social science is and what it can contribute to natural resource planning (Bennet et al., 2016; Miller et al., 2019).

Opportunities. The Public Engagement thematic group represents themes related to a critical insight derived from this study - socio-spatial data is public input. This is perhaps an obvious statement, but as these themes indicate, it has deeper implications. It determines where socio-spatial data will be collected and for what purpose; it affects how it is documented and integrated into the planning process. Socio-spatial data as public input represents possible leverage points within an on-going public engagement strategy and processes. Public outreach and engagement occurs all this time within forests, not just for plan revisions. Each engagement offers a new opportunity to collect socio-spatial data that can be compiled and documented for use in project-level planning, monitoring activities, public outreach and education. It need not be an isolated activity attached to a regimented NEPA process. However, it is likely that to expand opportunities (and realize the full potential of socio-spatial data to inform planning) will require that collection of socio-spatial data be embedded as a standard practice in land-use management.

Planning Strategies. The Planning Strategies theme connects to both the Staff Capacity and Public Engagement organizing themes. As the arrows indicate, each has a significant, but different, effect on selection of socio-spatial data tools, the kinds of data collected, and how that data is utilized. Limited staff capacity affects decisions made around prioritization of data collection tasks. This is evident in participants comments that refer to "low hanging fruit" or prioritizing collecting data that is required by 
legislation, such as the Endangered Species Act, at the expense of collecting other sociocultural or socio-spatial data. On the other side, the unique public engagement challenges and goals for each forest also affect which tools are selected and for what purpose. For example, the Flathead National Forest must navigate numerous potentially conflicting management priorities that have historically resulted in severe polarization among various stakeholder and interest groups. The forest chose to bring in an outside contractor to facilitate extensive outreach and public engagement around plan revision components. Numerous types of socio-spatial tools were used at strategic points in the process to inform stakeholders of management issues, collaborate in a way that supports consensus-building, and gauge public opinions around plan components.

Summary. This chapter has presented the themes with descriptive text segments and derived insights and questions. The socio-spatial thematic network provides a visualization that begins to tease out how these themes interact and influence each other within the wider system. As is the nature of exploratory research, the findings are both informative on their own to better understand structures and processes in place that either hinder or help in the collection and use of socio-spatial data, but also a means in which to encourage further inquiry. The next chapter explores implications of these findings for practitioners, researchers, policy-makers, and educators. 


\section{RECOMMENDATIONS}

This chapter synthesizes the study's findings into a set of recommendations for practitioners, researchers, educators and policy-makers. Much of the recommendations reflect suggestions made by study participants. Others draw from insights and questions posed in the discussion of the salient themes. Before presenting recommendations, however, the limitations of the study need to be made clear. The following section presents these limitations.

\subsection{Limitations of Study}

Revisiting the Research Question. Marshall and Rossman (2016) point out that it is not uncommon for exploratory research to result in challenging assumptions built into the research propositions. The impetus for this study came from a perceived reticence on the part of forest planners to take advantage of PPGIS-based HEM tools to collect socio-spatial data. GIS is a technology widely employed by natural resource scientists and sufficient evidence exists through numerous published case studies that tools such as HEM, VCA and SolVES can produce sophisticated and locally relevant GIS-ready data layers around public values. The bias embedded in this "perceived reticence" is that forest planners seemed not to be collecting socio-spatial data because they were not using these particular GIS tools. In light of the results of this study, this supposition was naive at best. I recognized my naiveté very early in the data collection process; the overarching research question was not unduly limiting. It asked what barriers and opportunities exist in using socio-spatial data, not in using GIS-based tools to collect socio-spatial data (an important follow-up question, but not one addressed in this study). As the study findings indicate, socio-spatial data is definitely being collected, 
interpreted and integrated into planning decisions using a wide variety of tools. Scholars and practitioners frustrated at the lack of adoption of sophisticated GIS-based tools to collect this kind of data should realize that it is not indicative of a rejection of the tool or the data it produces, but instead recognition that there are often intractable barriers to using these kinds of tools (namely OMB oversight, staff capacity and skillsets). As a footnote, TPCM was released just prior to the start of the data collection phase of this study and was not yet widely known or applied. However, those participants who had used TPCM indicated positive responses.

Inter-rater Reliability: Due to logistical issues and time constraints, inter-rater reliability was not conducted in this study. Inter-rater reliability is the degree of agreement between codes assigned to the same text segments by different persons. The higher the agreement, the more confidence that the coding structure adequately represents the dataset. In lieu of this, several strategies were applied in this study to strengthen the reliability and validity of the study design and results as suggested by Franklin et al. (2010). These include: (1) purposive sampling to target participants able to provide input relative to the research; (2) use of multiple data sources (interviews, field notes, formal documents); convergence of the findings from these sources increases validity; (3) structured protocols and codebooks reviewed by other researchers familiar with the subject matter; (4) use of multiple analytical tools for triangulation of results; (5) leaving an "audit trail" that provides information about decisions made at all points in the research process; this increases transparency and allows other researchers to gauge the robustness of the study design and interpretation of the results; and (6) creating "thick 
descriptions" that describe the process and report the outputs at each stage in an encyclopedic way.

Generalizability: The value of qualitative research is in revealing the breadth and nature of the phenomena under study, often by using a small set of participants or a single case study. This type of research, by its very nature, is not intended to provide statistical-probabilistic generalizability. The goal, particularly of case study research, is to examine a situation as understood best by those deeply involved. As Stake (2005) notes, qualitative inquiry is directed toward gathering information that has practical and functional uses rather than being overly concerned about formalizing a set of propositions or hypotheses. Toward that end, no claim is made that the findings presented here can be generalized beyond this particular dataset or USFS context. Emphasis is placed on identifying general themes that have practical application and that may inform further inquiry.

Confidentiality vs. Anonymity: All the USFS participants were interviewed in their professional capacity as employees of a public land management agency. This was made clear upfront in the invitation email as well as in the consent process. While participants were forthright and often critical at times, they were clearly and understandably careful about making any highly negative comments about the agency which they were representing. This study guaranteed confidentiality, but not anonymity. A study that guarantees anonymity may lead to different results than those reported here.

The Leadership Theme: All forest-level participants spoke highly of their forest leadership. This may be due to the purposive sampling strategy, in which forests that were stalled in their plan revision efforts were eliminated from consideration. Stalled 
plan revision may indicate a lack of effective or consistent leadership at the forest or regional level that is not reflected in this dataset. The leadership code applied in this study did not rise to the level of a strong primary theme, but rather served an explanatory function. Forest-level staff who indicated they received strong support from their forest supervisors felt empowered to apply different kinds of tools to collect socio-spatial data at numerous stages in the planning process.

These limitations are taken into consideration in the recommendations that follow. Care is taken not to make definitive statements or propositions that go beyond what can be gleaned from this particular dataset. However, as is the case for exploratory research, the themes elicited many questions deserving further inquiry. These questions are summarized with suggestions for further research.

\subsection{Recommendations for Practitioners}

All indications are that forest planners, whether their background is in the social or natural sciences, appreciate the value of socio-spatial data as being able to: (1) supplement regional socio-cultural and economic data gathered for the formal forest assessments with place-based data that reflect how local communities value, use and benefit from forest resources, and (2) better engage and secure buy-in from diverse stakeholder groups, treating them as critical partners in developing the need for change and desired conditions documents. Challenges exist with regard to data availability. The following list synthesizes suggestions from study participants on how to address sociospatial data gaps.

- Expand thinking about socio-spatial data as a multi-purpose tool and how it can be applied in numerous ways, such as public education or conflict resolution. 
- Collect socio-spatial data all the time, not just when needed for formal planning or projects; build it into standard forest management practices.

- Build collection of socio-spatial data into all public engagement activities, public outreach or education using maps to facilitate engagement, resolve conflicts or gather localized values and use-preferences.

- Use the model of participatory citizen science to assist in monitoring forest conditions and simultaneously gather information on how people are using or impacting resources and to engage and educate the community.

- Invest in TPCM; upgrade to increase its utility and ease of use and consider using the tool for meetings, outreach and education.

- Explore VGI (volunteered geographic information), crowdsourcing, cell phone applications, and geotagging, to reach a different kind of stakeholder than those who attend meetings.

\section{Rethinking collection of socio-spatial data as embedded in public engagement}

strategies. The first three ideas suggest expanding thinking around what socio-spatial data can offer to forest planners beyond data that is part of a formal NEPA process. These ideas advocate for thinking about socio-spatial data as a multi-purpose tool for public education, engagement or conflict resolution. This is data that can be collected at all points in an on-going public engagement strategy. It tells a story about the meaning of place and about local identity deeply embedded in that place that can be used to inform and guide management decisions. As one participant explained:

I really put a high value on the qualitative...I think what is really of value for our publics isn't the numbers, but rather the narrative and the interpretation of those numbers. It's talking about contributions to local communities like in terms of jobs, which is interesting, but in most cases, the agency contributes a pretty small portion of jobs to particular forest-related industries. That's not what people care about. People care about the fact that those jobs have been there for years and they're a part of their local identity. That's the qualitative interpretation of the importance of those jobs. That is what matters, not their small absolute value.

Socio-spatial data seen as a story serves a different, but no less valuable purpose.

It fills in knowledge gaps by reaching specific, perhaps small, cohorts of forest users to supplement what coarse quantitative datasets or generalized surveys done every five 
years cannot provide. For example, one study participant commented that decommissioning old logging roads is an important management task, but that task is very difficult and fraught with potential public outcry without sufficient knowledge about which roads locals are using to access forest resources. Others commented that they had little knowledge of what they call the "silent voices," local people who use the forest regularly, perhaps to gather firewood or harvest berries, but rarely attend any official meetings. Another large constituency are young adults and families, stakeholder groups that also do not regularly attend forest service public meetings or workshops. What does the forest mean to them? As one person lamented, and I paraphrase - if I don't know where they are going or what they are doing, I don't know how to protect the resources they depend on or the experience they seek.

Public engagement is a practice embedded in forest management. It does not end when a forest plan is finalized, but continues through monitoring activities, amendments to the plan, on-the-ground project implementation, educating the public about the forest, and outreach to the community of forest users, "friends of" collaboratives, and other interested constituencies. All of these activities are opportunities to collect valuable socio-spatial data through public engagement activities that are already occurring in multiple ways. As scholar, Anthony Cheng, notes:

That's really what resource management ought to be, connecting activities to real places and then how those places shape and reshape and affect how people interact with those places. When people attend a meeting, a standard Forest Service public meeting about what activities should occur where, people really gravitate towards things like maps and you can have a rich conversation about well, what do you do in this place; what does this place mean to you as a user, as a stakeholder, as a resource manager? And so the dialogue and the liberation occurs around a place rather than around rhetorical positions. [Cheng, A. 2-152015, personal interview, emphasis added] 
Expanding the socio-spatial data collection toolkit. The analysis presented in preceding chapters reveals a diversity of socio-spatial data collection tools that were used during the forest plan revision process, from simply locating special places on a static map to direct public interaction with and input into a PPGIS-based application such as TPCM or human ecology mapping. Participants offered several ideas on how to expand the toolkit even further to assist in reaching different kinds of forest user groups.

\section{Participatory citizen science, crowdsourcing and Volunteered Geographic}

Information (VGI). Citizen science, crowdsourcing and VGI are tools that are becoming much more accepted and mainstreamed. The Crowdsourcing and Citizen Science Act of 2016 (15 U.S.C. 3724) grants federal agencies explicit authority to pursue citizen science and crowdsourcing projects that assist in meeting agency mission and goals. Specifically, the Act recognizes the many benefits of crowdsourcing and citizen science projects including (1) accelerating scientific research; (2) maximizing the return on taxpayer dollars; (3) addressing societal needs; and (4) connecting members of the public directly to Federal science agency missions and to each other (15 U.S.C. $\S 3724(b)(2))$.

There is a lively debate within the academic literature on what is meant by citizen science (see Eitzel et al., 2017, for a comprehensive discussion). For the purposes here, the federal government defines citizen science as "a form of open collaboration in which individuals or organizations participate voluntarily in the scientific process in various ways" (15 U.S.C. $\$ 3724(c)(1))$. Crowdsourcing is a method to engage the public either informally or formally as part of a citizen science project. Crowdsourcing is defined as "a method to obtain needed services, ideas, or content by soliciting voluntary 
contributions from a group of individuals or organizations, especially from an online community" (15 U.S.C. $§ 3724(c)(2))$. VGI is a specific set of crowdsourcing applications that gather "geospatial content generated by non-professionals using mapping systems available on the internet" (as defined by the USGS, www.usgs.gov/core-science-systems/ngp/cegis/vgi). VGI is also referred to as the GeoWeb or Web 2.0.

To better understand and implement citizen science and crowdsourcing tools, the U.S. General Services Administration has an official website that provides information and a suite of tools to accelerate the use of crowdsourcing and citizen science across the U.S. government (available at www.citizenscience.gov). The site includes examples of federally-supported citizen science projects, a comprehensive toolkit on how to develop projects, and a gateway to a community of citizen science practitioners and coordinators across government agencies. Examples of USFS citizen science projects include:

1. Youth Forest Monitoring program at Helena-Lewis \& Clark national forest. This is a summer program where students collect forest health monitoring data and have contributed to establishing baselines for the forest revision process.

2. Neighbors to Nature project is a partnership between the Jackson Hole Wildlife Foundation, Bridger-Teton National Forest, Friends of Pathways and the Nature Conservancy's Wildflower Watch. This project engages the public in documenting wildlife activity, plant phenology, and trail use to better understand recreational patterns and ecological trends in lands near Jackson, Wyoming.

As a caution, Kar et al. (2016) note, while access to the public is made easier using the GeoWeb, care must be taken to avoid assigning more utility to VGI applications than is warranted. The GeoWeb can reach different people on certain topics of interest to them, but the reach is not universal so care must be taken in generalizing data too extensively. The authors also caution that while the GeoWeb can broaden 
recognition within the scientific community of new kinds of expertise, this still cannot replace collective engagement and political deliberation.

\section{Upgrading the Useability and Accessibility of TPCM and other PPGIS Tools.}

The USFS Talking Points Collaborative Mapping tool (TPCM) has the capability to collect socio-spatial data by attaching use- and values-based public comments to specific geographic locations. The more it is used, in data collection tasks beyond forest plan revision, and its utility documented, the more likely the agency will increase its investment in upgrading, technical support, staff training, and dissemination of best practice information. For practitioners, be creative in identifying areas in which sociospatial data can be collected. Use the TPCM tool prolifically, not just in plan revision, but also at the project level and for information sessions or workshops offered to the public. Seek out partnerships, with universities and community organizations, to explore opportunities for more sophisticated human ecology mapping applications. These are especially helpful in gathering targeted data for projects such as road decommissioning and other issues that require more robust data than TPCM can provide (Cerveny et al., in press).

There needs to be a critical mass of persons employing socio-spatial data collection tools in order to embed the practice into standard planning and management processes. To reach that critical mass, practitioners should use TPCM and other HEM mapping tools whenever and wherever possible. Partnerships with universities can mitigate internal capacity issues (student labor is very cost-effective); partnerships with community-based organizations can reach a targeted constituency (use their online platform to reach their members). Leverage these efforts and eke out time to write a 
technical brief or report and disseminate on the agency website and presentations at professional meetings. Champions of socio-spatial data will gravitate toward expanding its collection and use. Skeptics will require evidence of its value and utility. Document what data was collected and how it was utilized in day-to-day operations or to address planning or monitoring challenges. Making it personal is helpful. Focus on how sociospatial data might allow someone to display excellence in executing their tasks, such as providing a planner with valuable information about backcountry use to help in monitoring environmental conditions or presenting a socio-spatial dataset that can mitigate potential public outcry on a management decision (what roads NOT to decommission).

\subsection{Recommendations for Researchers}

Revisiting Contingency Theory and Equifinality. In inductive research, insights gleaned from themes are often the catalyst for interesting questions or observations not anticipated in the original conceptual framework or design of the interview protocols (which leaves many questions unanswered). Consistent threads that permeate the themes presented here are: (1) the choices and decisions being made around allocation of limited resources (staff time and budget); (2) trade-offs that may be occurring and potential impacts on the agency's ability to collect and use socio-spatial data in forest planning and/or the quality of the assessments produced; and (3) the intersection between socio-spatial data and the public engagement process. The framework and concepts provided by contingency theory provide a useful way to explore this thread through additional scientific inquiry. 
In review, contingencies are internal and external factors the organization is responding and adjusting to in its efforts to achieve optimal performance. "Best fit" is achieved internally (horizontal congruence) when the organization's practices, strategies and structures work together to achieve a desired outcome, and externally (vertical congruence) when practices and strategies of the organization match the environmental conditions in which it operates. The concept of equifinality states that an organization will perform effectively if the critical functions it must carry out, as determined by the environment, are met by its organizational structures and this can be achieved via numerous configurations. The suboptimal equifinality proposition says that in cases of multiple conflicting functions, managers will resolve contingency conflicts by focusing on a single, dominant function and performance around other functions will be suboptimal (see Chapter 3 for more detailed information).

There is some indication that the USFS is in a suboptimal scenario. As an agency, it must find ways to balance often competing legal mandates, such as providing broad public access to forest resources while also protecting habitat for endangered species, or creating structures that comply with heavily prescribed NEPA processes while also being flexible and accommodating in the face of contentious public attitudes around land-use preferences. Designing a research study within the equifinality framework will help to shed light on what trade-offs might be occurring, either explicitly or implicitly, and their impact on functional performance (how does it affect the ability of the agency to collect and effectively integrate socio-spatial data into a forest plan?).

Some external and internal contingencies identified in the findings are listed below as well as a few instances where there is a possibility a trade-off is occurring. 
These contingencies are by no means exhaustive, but indicate the most salient revealed

by this study. This is followed by a list of questions synthesized from the findings.

\section{External Contingencies:}

1. PRA/OMB oversight.

2. Planning Rule protocols for types of assessments required in the forest plan.

3. Mandates around "existing" data.

4. Budget appropriation.

5. Political/public pressures (efficiency $\&$ cost-effectiveness in conducting plan revision vs. flexibility $\&$ accommodation toward public values/preferences).

\section{Internal Contingencies:}

1. Science- or data-driven culture manifest in organizational structures and processes.

2. Employee incentives (e.g. research scientists \& published works).

3. Budgetary decisions.

4. Decision matrices (NEPA compliance).

5. Staffing, staff capacity, and task assignments.

\section{Trade-Off Scenarios:}

1. Research Station Scientists: Publish in academic journals or write a technical brief for on-the-ground planners?

2. Potential Deprioritization of Socio-Spatial Data: Is "hard" science being prioritized over public values?

3. The "Low Hanging Fruit" Scenario: Limited staff capacity and funds require making decisions about what socio-spatial data can be collected and lead to relying on coarse available data (U.S. Census; U.S. Dept. of Labor databases) that provides very little information about public values and uses at the local level.

\section{Compelling Questions Posed in Themes:}

1. Do organizational decisions about the level of socio-spatial data collection (and allocation of resources toward that purpose) reflect a trade-off? If so, how does that affect the desired quality of socio-cultural assessments in the planning context? Does it represent suboptimal equifinality?

2. To what extent do decision-makers view forest planning (particularly with regard to comprehensive and project-level plans) through the lens of NEPA compliance (a rigid external contingency)? Is there evidence of a correlation between a high focus on NEPA-based decision-making and lower levels of socio-spatial data collection?

3. Budget allocations are an important contingency within organizations. Where does discretion lie within the decision-making protocols and processes? Are budget allocations aligned with the functions the organization must perform to produce a forest plan of desired quality? 
4. Does lack of access to current research findings hinder a forest planner's ability to make well-informed decisions (this reflects the need for a data portal)?

The challenges evident in the themes seem to hint that there is misalignment between structure (e.g. allocation of resources) and functions (e.g. collection of sociospatial data) that result in less than adequate (or suboptimal) outcomes, particularly in meeting the stated goals of meaningful public input and integration of public values into the forest management plan (e.g. the "low hanging fruit" trade-off).

As the study documented here is exploratory, the data is not nuanced enough to make definitive statements as to the nature of the trade-offs or the consequences in organizational performance or the quality of outputs that these trade-offs might produce. Constructing a research project configured around suboptimal equifinality and focusing on the nature of trade-offs or impacts will refine the thematic network and expose more linkages and loops between elements representing barriers, challenges and opportunities.

Forest Comparative Analysis. The adage "if I knew then what I know now" is apropos in exploratory research. Extrapolation of themes from the dataset reveal compelling questions that cannot be answered using the initial dataset. One cannot help but wish more probing interview prompts would have been employed. A comparative study with a larger forest sample that reaches many more on-the-ground planners can dive deeper and explore more nuanced aspects of the factors impacting the collection and use of socio-spatial data. This would be beneficial to uncover additional factors, correlations with and linkages between external contingencies or internal organizational structural elements, and causal factors or feedback loops that impact decisions made during the planning process. Questions of interest may include: 
1. What are attitudes about the role of the public in forest planning (is it contentious or collaborative?); does this affect socio-spatial data collection priorities?

2 . Is there a correlation between collection and use of socio-spatial data and particular management challenges or priorities (do recreation-heavy forests collect - or want to collect - socio-spatial data more than forests with other types of priorities)?

3. What are other structures, processes or protocols put in place by forests that reduce (or create) barriers to collecting socio-spatial data?

A comparative study would contribute to the literature on USFS (and other federal agency) structure and culture that have explored this topic in a number of contexts, such as community collaboration (Orth, 2015), public NEPA commenting (DéArman, 2020), sustainability (Ma et al., 2020) and leadership (Morgan et al., 2018).

\subsection{Recommendations for Policy}

The role of policy is another avenue for improving the collection and use of sociospatial data. Policy is a formal system of principles that guide organizational decisions to achieve desired outcomes. Policies are operational in nature; they are statements of intent that translate into protocols. In this way, policy influences practice in often powerful ways (Dunn, 2012).

A plethora of statements from the USDA and the USFS extoll the value of human-environment connections and the importance of understanding values the public holds for special places and landscapes (e.g. the Forest Service Social Science Research Agenda, the USDA Forest Service Strategic Plan, and numerous agency general technical briefs). However, these statements have not translated well into specific policy directives around public engagement or collection of public values-based data, nor are they operationalized in the Forest Service Directives. Any formal policy that directly addresses social science, socio-spatial data and/or public engagement is a validation 
statement and adds a new factor that leaders and decision-makers must consider as they allocate budgets, assign staff time and determine planning and management priorities.

While this may seem like a rather naïve suggestion, effective policy around sociospatial data need not come from the highest levels. Forest supervisors have a great deal of latitude in how they manage the internal operations of their forest, as do regional foresters. A simple internal policy statement, that says something like "the Flathead National Forest has adopted the use of TPCM to collect public input on values and uses of our forest resources," is a policy statement of few words, but with potentially high impact. Built into this policy statement is a commitment to invest in building staff capacity to employ the tool and to use the socio-spatial data it produces (where appropriate, of course). Policy can accelerate adoption of practices. And, when an adopted policy is effective in achieving a desired outcome, it tends to expand upward and outward. When a forest ranger secures a supervisor position in a different forest, they are likely to take successful policies and practices with them.

\subsection{Recommendations for Educators and other Professionals}

Educators and other professionals also have a role to play in advocating for increased use of socio-spatial data in natural resource planning. Educators can advocate for development of courses (or sections within courses) and professional development opportunities that focus on public values in natural resource planning and highlight the utility of socio-spatial data in this context. Such courses will expose students to the numerous socio-spatial data collection tools that are available and areas in which the data has been applied in land-use decisions. 
There is a lack of skills and experience needed to collect and utilize socio-spatial data for natural resource planning and management, especially among practitioners working on the ground (Kahila-Tani et al., 2016). Landscape architecture specialists are often tasked in areas such as recreation planning and will be asked to glean data from surveys such as National Visitor Use Monitoring Survey or create analyses from the Recreation Opportunity Spectrum or Scenic Management System. Public affairs staff develop public outreach and engagement strategies. These two specialty areas need to work closely together to maximize opportunities to collect socio-spatial data via public engagement activities and integrate it into their work, but cannot do so routinely without the requisite knowledge and skills. Staff hired to work in natural resource management and environmental science would also benefit from an appreciation of the insights that socio-spatial data can provide when trying to balance public values and land-use priorities. Offering optional courses, sections within existing courses, or professional development opportunities for those working in the fields of natural resource management, landscape architecture, environmental science, human and physical geography, public affairs and policy, urban planning and many other fields would help to create a workforce that can see the collection and use of socio-spatial data as a best practice in natural resource management, rather than something extraneous that may be of help in a plan revision or a project-level NEPA process.

For professionals who are on planning boards for professional associations, consider advocating for special sessions at professional conferences focusing on public values and socio-spatial data applications within the broad context of urban and/or natural resource planning, ecosystem services, collaborative governance, environmental 
leadership, and the like. Constantly reinventing the wheel is inefficient. There is much work around public values and incorporation of socio-spatial data being conducted on numerous fronts and it will only expand as more agencies and organizations adopt PPGIS, crowdsourcing and VGI tools. Special forums would make this work more visible and consolidated (this is preferable to presentations being peppered throughout a conference schedule). A special forum would attract a cadre of practitioners and researchers that can share ideas and best practices. There are several professional associations which might host such a session, such as the International Association for Society and Natural Resources, American Association of Geographers, International Association of Landscape Ecology, Society for Urban Ecology, the Society of American Foresters, among others.

Conclusion. The spectrum of socio-spatial data is wide. It can be as simple as a conversation about a special place to creating GIS-ready data layers that can be incorporated into sophisticated modelling software. However, the purpose is the same to make tangible public values around public places so that greater understanding leads to better land-use decisions. The goal of this study was to tease out what elements represent barriers and opportunities. Socio-spatial data is both a tool (data) and a practice (incorporation of public values into analysis and decision processes). My hope is that the themes presented here will lead others to think about and develop strategies that enhance the utility and realize the potential of socio-spatial data to engage the public and infuse their sense of place into land management decisions. 


\section{REFERENCES}

Adams, K. M. (2012). Systems theory: a formal construct for understanding systems. International Journal of Systems and Systems Engineering 3(3/4): 209-224.

Alessa, L., Kliskey, A., \& Brown, G. (2008). Social-ecological hotspots mapping: a spatial approach for identifying coupled social-ecological space. Landscape and Urban Planning 85(1): 27-39.

Allan, C., Curtis, A., Stankey, G. \& Shindler, B. (2008). Adaptive management and watersheds: a social science perspective. Journal of the American Water Resources Association 44(1): 166-174.

Allen, S. D., Wickwar, D. A., Clark, F. P., Dow, R. R., Potts, R. \& Snyder, S. A. (2009). Values, Beliefs, and Attitudes Technical Guide for Forest Service Land and Resource Management, Planning, and Decision-making. Portland OR: U.S. Department of Agriculture, Forest Service, Pacific Northwest Research Station (PNW-GTR-788).

Altman. I. \& Low. S. M. (1992). Place Attachment. Boston, MA: Springer.

Ambrose-Oji, B. \& Pagella, T. (2012). Spatial Analysis and Prioritisation of Cultural Ecosystem Services: A Review of Methods. Alice Holt Lodge: Farnham, Surrey UK.

Anstedt, S. (2010). Challenges to implementing "Best Available Science." JFSP Briefs (124). Available at www.firescience.gov (last accessed Oct. 2020).

Aran, E. \& Reed P. (2015). Talking Points Collaborative Mapping: Forest Service Public Participation GIS Application. Paper presented at the ESRI User Conference, July 20-24, 2015, San Diego, CA. Available at https://proceedings.esri.com/library/userconf/proc15/papers/890_503.pdf.

Arnold, R. D. \& Wade, J. P. (2015). A definition of systems thinking: a systems approach. Procedia Computer Science 44: 669-678.

Attride-Stirling, J. (2001). Thematic networks: an analytic tool for qualitative research. Qualitative Research 3(3): 385-405.

Ban, N. C., Mills, M., Tam, J., Hicks, C. C., Klain, S. C., Stoeckl, N., Bottrill, M.C., Levine, J., Pressey, R.L., Satterfield, T. Chan, K. M. A. (2013). A social-ecological approach to conservation planning: embedding social considerations. Frontiers in Ecology \& the Environment 11(4): 194-202.

Barbier, E. B. (2010). Scarcity and Frontiers: How Economies Have Developed Through Natural Resource Exploitation. New York: Cambridge University Press. 
Barbour, J. (2007). Accelerating Adoption of Fire Science and Related Research. JFSP Research Project Reports (97). Available at http://digitalcommons.unl.edu/jfspresearch/97 (last accessed July, 2021).

Battilana, J. \& Casciaro, T. (2012). Change agents, networks, and institutions: a contingency theory of organizational change. The Academy of Management Journal 55(2): 391-398.

Bauer, J. W. R., Ries, P., \& Rosenberger, R. S. (2020). A relationship between emotional connection to nature and attitudes about urban forest management. Urban Ecosystems 23(1): 187-197.

Bennett, N. J., Roth, R., Klain, S. C., Chan, K. M. A., Clark, D. A., Cullman, G., Epstein, G., Nelson, M.P., Stedman, R., Teel, T. L., Thomas, R. E. W., Wyborn, C., Curran, D., Greenberg, A., Sandlos, J. \& Verissimo, D. (2016). Mainstreaming the social sciences in conservation. Conservation Biology 31(1): 56-66.

Biedenweg, K., Cerveny, L. \& McLain, R. (2014). Values mapping with Latino forest users: contributing to the dialogue on multiple land use conflict management. Practicing Anthropology 36(1): 33-37.

Biedenweg, K. \& Nelson, M. P. (2017). Demystifying the human dimension of ecological research. Frontiers in Ecology and the Environment 15(3): 119.

Birkinshaw, J., Nobel, R. \& Ridderstrale, J. (2002). Knowledge as a contingency variable: do the characteristics of knowledge predict organization structure? Organization Science 13(3): 274-289.

Black, A. E. \& Liljeblad, A. (2008). Integrating Social Values in Vegetation Models via GIS: The Missing Link for the Bitterroot National Forest. Final Report to the Joint Fire Science Program (JFSP Project No. 04-2-1-114).

Blake, D., Auge, A. A. \& Sherren, K. (2017). Participatory mapping to elicit cultural coastal values for Marine Spatial Planning in a remote archipelago. Ocean \& Coastal Management 148: 195-203.

Braun, V. \& Clarke, V. (2006). Using thematic analysis in psychology. Qualitative Research in Psychology 3(2): 77-101.

Bright, A. D., Cordell, H. K., Hoover, A. P. \& Tarrant, M. A. (2003). A Human Dimensions Framework: Guidelines for Conducting Social Assessments. Asheville, NC: U.S. Department of Agriculture, Forest Service, Southern Research Station (Gen. Tech. Rep. SRS-65). 
Brown, G. \& Donovan, S. (2014). Measuring change in place values for environmental and natural resource planning using public participation GIS (PPGIS): results and challenges for longitudinal research. Society \& Natural Resources 27(1): 36-54.

Brown, G., \& Fagerholm, N. (2015). Empirical PPGIS/PGIS mapping of ecosystem services: a review and evaluation. Ecosystem Services 13: 119-133.

Brown, G. \& Raymond, C. M. (2007). The relationship between place attachment and landscape values: Toward mapping place attachment. Applied Geography 27(2): 89111.

Brown, G., \& Reed, P. (2000). Validation of a forest values typology for use in national forest planning. Forest Science 46(2): 240-247.

Brown, G. \& Reed, P. (2009). Public participation GIS: a new method for national forest planning. Forest Science 55(2): 166-182.

Brown, G. \& P. Reed (2012). Values compatibility analysis: using public participation geographic information systems (PPGIS) for decision support in national forest management. Applied Spatial Analysis \& Policy 5(4): 317-322.

Brown, G., Reed, P. \& Raymond, C. M. (2020). Mapping place values: 10 lessons from two decades of public participation GIS empirical research. Applied Geography 116: 102156.

Brown, G., Squirrell, T. G., \& Harris, C. C. (2010). Growing organizational challenges for the U.S. Forest Service: results of a longitudinal study in a period of major environmental change. Journal of Forestry 108: 77-85.

Bryan, B. A., Raymond, C. M., Crossman, N. D. \& King, D. (2010). Comparing spatially explicit ecological and social values for natural areas to identify effective conservation strategies. Conservation Biology 25(1): 172-181.

Buzon, T. (2018). Mind Map Mastery. London, UK: Watkins Publishing.

Cerveny, L. K., McLain, R. J., Rohdy, S. (in press). The use of socio-spatial data for sustainable roads planning: a national forest case study. Journal of Environmental Planning and Management (in press).

Charnley, S., Carothers C., Satterfield, T., Levine, A., Poe, M.R., Norman, K., Donatuto, J. Breslow, S. J., Mascia, M. B., Levin, P. S., Basurto, X., Hicks, C. C., GarciaOuijano, C. \& St. Martin, K. (2017). Evaluating the best available social science for natural resource management decision-making. Environmental Science \& Policy 73: $80-88$. 
Cheng, A. S. \& Daniels, S. E. (2003). Examining the interaction between geographic scale and ways of knowing in ecosystem management: a case study of place-based collaborative planning. Forest Science 49(6): 841-854.

Cheng, A. S., Kruger, L. E. \& Daniels, S. E. (2003). "Place" as an integrating concept in natural resource politics: propositions for a social science research agenda. Society \& Natural Resources 16(2): 87-104.

Cheng, A.S. \& Mattor, K.M. (2010). Place-based planning as a platform for social learning: insights from a national forest landscape assessment process in western Colorado. Society and Natural Resources 23(5): 385-400.

DéArmon, K. J. (2020). Is public participation public inclusion? The role of comments in US Forest Service decision-making. Environmental Management 66(1): 91-104.

Donaldson, L. (2001). The Contingency Theory of Organizations. Thousand Oaks, CA: Sage Publications.

Drazin, R. \& Van de Ven, A. H. (1985). Alternative forms of fit in contingency theory. Administrative Science Quarterly 30(4): 514-539.

Dunn, C. E. (2007). Participatory GIS: a people's GIS? Progress in Human Geography 31(5): 616-637.

Dunn, W. (2012). Public Policy Analysis, $5^{\text {th }}$ edition. New York: Pearson Publishing.

Eitzel, M. V., Cappadonna, J. L., Santos-Lang, C., Duerr, R. E., Virapongse, A., West, S. E., Kyba, C. C. M., Bowser, A., Cooper, C. B., Sforzi, A., Metcalfe, A. N. Harris, E. S. Thiel, M., Haklay, M., Ponciano, L., Roche, J., Ceccaroni, L., Shilling, F. M., Dorler, D., Heig, F., Kiessling, T., Davis, B. Y. \& Jiang, Q. (2017). Citizen science terminology matters: exploring key terms. Citizen Science Theory and Practice 2(1): $1-20$.

Elwood, S. (2006). Negotiating knowledge production: the everyday inclusions, exclusions, and contradictions of participatory GIS research. Professional Geographer 58(2): 197-208.

Emery, F. E. \& Trist, E. L. (1965). The causal texture of organizational environments. Human Relations 18(1): 21-32.

Fagerholm, N., Eilola, S., Kisanga, D., Arki, V. \& Käyhkö, N. (2019). Place-based landscape services and potential of participatory spatial planning in multifunctional rural landscapes in southern highlands, Tanzania. Landscape Ecology 34: 1769-1787. 
Fagerholm, N. \& Kayhko, N. (2009). Participatory mapping and geographical patterns of the social landscape values of rural communities in Zanzibar, Tanzania. Fennia 187(1): 43-60.

Fagerholm, N., Raymond, C. M., Olafsson, A. S., Brown, G., Rinne, T., Hasanzadeh, K., Broberg, A. \& Kyttä, M. (2021). A methodological framework for analysis of participatory mapping data in research, planning, and management. International Journal of Geographical Information Science. Available online at: Full article: A methodological framework for analysis of participatory mapping data in research, planning, and management (tandfonline.com).

Farnum, J., Hall, T. \& Kruger, L. E. (2005). Sense of Place in Natural Resource Recreation and Tourism: An Evaluation and Assessment of Research Findings. Portland, OR: U.S. Department of Agriculture, Forest Service, Pacific Northwest Research Station (PNW-GTR-660).

Felicio, T., Samagaio, A. \& Rodriguez, R. (2021). Adoption of management control systems and performance in public sector organizations. Journal of Business Research 124: 593-602.

Fielder, F. E. (1967). A Theory of Leadership Effectiveness. New York: McGraw Hill.

Fisher, J.G. (1998). Contingency theory, management control systems and firm outcomes: past results and future directions. Behavioral Research in Accounting 10: 47-64.

Franklin, C. S., Cody, P. A., \& Ballan, M. (2010). Reliability and validity in qualitative research. In: Thyer, B. A., The Handbook of Social Work Research Methods, 2nd Edition. Thousand Oaks, CA: Sage Publications.

Francis Marion National Forest (2013). Francis Marion National Forest Draft Forest Plan Assessment. U.S. Department of Agriculture, U.S. Forest Service.

Galletta, A. (2013). Mastering the Semi-Structured Interview and Beyond. New York: New York University Press.

Galliano, S. J. \& Loeffler, G. M. (1999). Place Assessment: How People Define Ecosystems. Portland, OR: U.S. Department of Agriculture, Forest Service, Pacific Northwest Research Station (GTR-PNW-462).

Gibbs, G. R. (2012). Thematic coding and categorizing. In: Gibbs, G. R. Analyzing Qualitative Data. Thousand Oaks, CA: Sage Publications, Inc., pp. 54-71. 
Goodchild, M. F., Anselin, L., Appelbaum, R. P. \& Harthorn, B. H. (2000). Toward spatially integrated social science. International Regional Science Review 23(2): 139159.

Gresov, C. \& Drazin, R. (1997). Equifinality: functional equivalence in organization design. The Academy of Management Review 22(2): 403-428.

Guest, G., MacQueen, K. M. \& Namey, E. E. (2012). Applied Thematic Analysis. Thousand Oaks, CA: Sage Publications, Inc.

Hall, T. E., Farnum, J. O., Slider, T. C. \& Ludlow, K. (2009). New Approaches to Forest Planning: Inventorying and Mapping Place Values in the Pacific Northwest Region. Portland, OR: U.S. Department of Agriculture, Forest Service, Pacific Northwest Research Station (PNW-RN-562).

Hartley, J. (2004). Case study research. In: Cassell, C. \& Symon, G. (eds). Essential Guide to Qualitative Methods in Organizational Research. London: Sage Publications Ltd., pp. 323-333.

Haynes, R. W. (2005). Developing an Agenda to Guide Forest Social Science, Economics, and Utilization Research. Portland OR: U.S. Department of Agriculture, Forest Service, Pacific Northwest Research Station (PNW-GTR-627).

Hays, S.P. (2009). The American People and the National Forests: The First Century of the U.S. Forest Service. Pittsburgh, PA: University of Pittsburgh Press.

Hoover, K. \& Stern, M. J. (2014). Constraints to public influence in U.S. Forest Service NEPA processes. Journal of Environmental Planning and Management 57(2): 173189.

Jankowski, P., Forss, K., Czepkiewicz, M., Saarikoski, H. \& Kahila M. (2021). Assessing impacts of PPGIS on urban land use planning: evidence from Finland and Poland. European Planning Studies (Published online: Assessing impacts of PPGIS on urban land use planning: evidence from Finland and Poland: European Planning Studies: $\underline{\text { Vol 0, No } 0 \text { (tandfonline.com) }}$

Jennings, D. F. \& Seaman, S. L. (1994). High and low levels of organizational adaptation: an empirical analysis of strategy, structure, and performance. Strategic Management Journal 15(6): 459-475.

Johnson, O. (2019). General system theory and the use of process mining to improve care pathways. In: Scott, P., de Keizer, N. \& Georgiou, A. (eds). Applied Interdisciplinary Theory in Health Informatics: A Knowledge Base for Practitioners. Washington, D.C.: IOS Press. 
Kahila-Tani, M., Broberg, A., Kyttä, M. \& Tyger, T. (2016). Let the citizens mappublic participation GIS as a planning support system in the Helsinki master plan process. Planning Practice and Research 31(2): 195-214.

Kaiser, B. A. (2006). The National Environmental Policy Act's influence on USDA Forest Service decision-making, 1974-1996. Journal of Forest Economics 12: 109130.

Kar, B., Sieber, R., Haklay, M. \& Ghose, R. (2016). Public participation GIS and participatory GIS in the era of GeoWeb. The Cartographic Journal 53(4): 296-299.

Kangas, A., Haapakoski, R. \& Tyrvainen, L. (2008). Integrating place-specific social values into forest planning - case of UPM-Kymmene Forests in Hyrynsalmi, Finland. Silva Fennica 42(5): 773-790.

Kathuria, R., Maheshkumar, J. P., \& Porth, S. J. (2007). Organizational alignment and performance: past, present and future. Management Decision 45(3): 503-51.

Kaufmann, M. R., Graham, R. T., Boyce, D.A., Moir, W. H., Perry, L., Reynolds, R. T., Bassett, R. L., Mehlhop, P., Edminster, C. B., Block, W. M., Corn, P. S. (1994). An Ecological Basis for Ecosystem Management. Fort Collins, CO: U.S. Department of Agriculture, Forest Service, Rocky Mountain Forest \& Range Experiment Station (RM-GTR-246).

Klein, P. G., Mahoney, J. T., McGahan, A. M. \& Pitelis, C. N. (2019). Organizational governance adaptation: who is in, who is out, and who gets what. Academy of Management Review 44(1): 6-27.

Koontz, T. M. (2002). Federalism in the Forest: National versus State Natural Resource Policy. Washington, DC: Georgetown University Press.

Kotus, J. \& Rzeszewski, M. (2020). Online mapping platforms: between citizen-oriented and research-focused tools of participation? Journal of Planning Education and Research. Online at: https://doi-org.proxy.lib.pdx.edu/10.1177/0739456X20903099.

Kruger, L. E. \& Jakes, P. J. (2003). The importance of place: advances in science and application. Forest Science 49(6): 819-821.

Kruger, L. E. \& Williams, D. R. (2007). Place and place-based planning. In: Proceedings of the National Workshop on Recreation Research and Management. Portland OR: U.S. Department of Agriculture, Forest Service, Pacific Northwest Research Station (PNW-GTR-698). 
Lebel, L., Anderies, J. M., Campbell, B., Folke C., Hatfield-Dodds, S., Hughes, T. P. \& Wilson, J. (2006) Governance and the capacity to manage resilience in regional social-ecological systems. Ecology and Society 11(1): 19-40.

Ma, Z., Steele, D., Cutler, A. \& Newcomb, K. (2020). Promoting sustainability in public natural resource agencies: insights from the USDA Forest Service. Journal of Forestry 118(2): 105-123.

MacQueen, K. M., McLellan, E., Kelly, K. \& Milstein, B. (2008). Codebook development for team-based qualitative analysis. In: Krippendorff, K. \& Bock, M.A. The Content Analysis Reader. Thousand Oaks, CA: Sage Publishing, Inc. pp. 211219.

Manfredo, M. J., Vaske, J. J., Rechkemmer, A. \& Duke, E. A. (2014). Understanding Society and Natural Resources: Forging New Strands of Integration Across the Social Sciences. New York, NY: International Association for Society and Natural Resources.

Marshall, C. \& Rossman, G. B. (2016). Designing Qualitative Research, 6th Edition. Thousand Oaks, CA: Sage Publications, Inc.

McCall, M. K. \& Dunn, C. E. (2012). Geo-information tools for participatory spatial planning: fulfilling the criteria for 'good' governance? Geoforum 43(1): 81-94.

McDonagh, J. and Tuulentie, S., eds. (2020). Sharing Knowledge for Land Use Management. Northampton, MA: Edward Elgar Publishing, Inc.

McLain, R., Cerveny, L., Besser, D., Banis, D., Biedenweg, K., Todd, A. N., KimballBrown, C. \& Rohdy, S. (2013). Mapping Human-Environment Connections on the Olympic Peninsula: An Atlas of Landscape Values. Portland, OR: U.S. Department of Agriculture, Forest Service.

McLain, R., Poe, M., Biedenweg, K., Cerveny, L. Besser, D. \& Blahna, D. (2013). Making sense of human ecology mapping: an overview of approaches to integrating socio-spatial data into environmental planning. Human Ecology 41(5): 651-665.

Miles, M. B. \& Huberman, A. M. (1994). Qualitative Data Analysis: An Expanded Sourcebook, 2nd Edition. Thousand Oaks CA: Sage Publications, Inc.

Miller, M.L., Gale, R. P. \& Brown, P. J. (eds). (2019). Social Science in Natural Resource Management Systems. New York: Routledge.

Mintzberg, H. (1983). Structure in Fives: Designing Effective Organizations. Englewood Cliffs, NJ: Prentice Hall. 
Mitchell, M. Y., Force, J.E., Caroll, M. S. and McLaughlin, W. J. (1993). Forest places of the heart: Incorporating special paces into public management. Journal of Forestry 91(4):32-37.

Moon, K. \& Blackman, D. (2014). A guide to understanding social science research for natural scientists. Conservation Biology 28(5): 1167-1177.

Morgan, D. F., Ingle, M. D. \& Shinn, C. W. (2018). New Public Leadership: Making a Difference from Where We Sit. New York: Routledge.

Morrison, A. B. (1986). OMB interference with agency rulemaking: the wrong way to write a regulation. Harvard Law Review 99(5): 1059-1074.

Namey, E., Guest, G., Thairu, L. \& Johnson, L. (2008). Data reduction techniques for large qualitative datasets. In: Guest, G. \& MacQueen, K. M. (eds). Handbook for Team-Based Qualitative Research. New York: Altamira Press, pp. 137-162.

Nie, M. (2011). Place-based national forest legislation and agreements: common characteristics and policy recommendations. Environmental Law Reporter 41: 10229-10246.

Nielsen-Pincus, M. (2011). Mapping a values typology in three counties of the interior northwest, USA: scale, geographic associations among values, and the use of intensity weights. Society \& Natural Resources 24(6): 535-552.

Orth, P. B. (2015). Organizational Change in the United States Forest Service: The Role of Community Collaboration. Ph.D. Dissertation, Department of Forest and Rangeland Stewardship, Colorado State University, Fort Collins, CO.

Pennings, J. M. (1998). Structural Contingency Theory. In: Drenth, P. J. D., Thierry, P. $\&$ de Wolff, C. J. (eds). Organizational Psychology. East Sussex, UK: Psychology Press Ltd.

Pfeffer, K., Baud, I., Denis, E., Scott, D. \& Sydenstricker-Neto, J. (2013). Participatory spatial knowledge management tools: empowerment and upscaling or exclusion? Information, Communication \& Society 16(2): 258-285.

Pinchot, G. (1947, commemorative edition). Breaking New Ground. Wash., D.C.: Island Press.

Proshansky, H. M., Fabian, A. K., \& Kaminoff, R. (1983). Place identity: physical world socialization of the self. Journal of Environmental Psychology 3: 57-83.

Radil, S. M. \& Anderson, M. B. (2018). Rethinking PGIS: participatory or (post)political GIS? Progress in Human Geography 43(2): 195-213. 
Raymond, C. M. \& Brown, G. (2007). A method for assessing protected area allocations using a typology of landscape values. Journal of Environmental Planning and Management 49(6): 797-812.

Relyea, H. C. (2000). Paperwork Reduction Act reauthorization and government information management issues. Government Information Quarterly 17(4): 367-393.

Robinson, P., Genskow, K., Shaw, B. \& Shepard, R. (2012). Barriers and opportunities for integrating social science into natural resource management: lessons from national estuarine research reserves. Environmental Management 50(6): 998-1011.

Rose, L., Hallemeier, J. \& Colburn, K. (2020). National forest planning: applying new technologies and approaches to improve public participation and decisionmaking. In: Selin, S., Cerveny, L. K., Blahna, D. J. \& Miller, A. B., eds. (2019). Igniting Research for Outdoor Recreation: Linking Science, Policy, and Action. Portland, OR: U.S. Department of Agriculture, Forest Service, Pacific Northwest Research Station (GTR-PNW-987).

Ruiz-Frau, A., Edwards-Jones, G. \& Kaiser, M. J. (2011). Mapping stakeholder values for coastal zone management. Marine Ecology Progress Studies 434: 239-249.

Ryan, G. W. \& Bernard, H. R. (2003). Techniques to identify themes. Field Methods 15(1): 85-109.

Saldana, J. (2013). The Coding Manual for Qualitative Researchers (2nd ed.). London: SAGE Publications Ltd.

Schein, E. H. (2010). Organizational Culture and Leadership (4th edition). San Francisco, CA: Jossey Bass.

Sherrouse, B. C., Clement, J. M. \& Semmens, D. J. (2011). A GIS application for assessing, mapping, and quantifying the social values of ecosystem services. Applied Geography 31(2): 748-760.

Sieber, R. (2006). Public participation geographic information systems: a literature review and framework. Annals of the Association of American Geographers 96(3): 491-507.

Skyttner, L. (2015). General System Theory: Problems, Perspectives, Practice, 2nd Edition. Hackensack, NJ: World Scientific Publishing Company.

Stake, R. E. (2005). Qualitative case studies. In N. K. Denzin \& Y. S. Lincoln (eds.), The Sage Handbook of Qualitative Research (p. 443-466). Thousand Oaks, CA: Sage Publications Ltd. 
Stedman, R. C. (2003). Is it really just a social construction? The contribution of the physical environment to sense of place. Society \& Natural Resources 16(8): 671-685.

Strang, V. (2009). Integrating the social and natural sciences in environmental research: a discussion paper. Environment, Development \& Sustainability 11(1): 1-18.

Suharyanto, A. \& Dwi Lestari, R. (2020). The fall and rise of the contingency theory of leadership. Proceedings of the IAPA Annual Conference, Dec. 2020, pp. 479-496.

Szymkowiak, M. (2021). A conceptual framework for incorporating human dimensions into integrated ecosystem assessments. Frontiers in Marine Science 8: 1-14.

Thomas, D. R. (2006). A general inductive approach for analyzing qualitative evaluation data. American Journal of Evaluation 27(2): 236-246.

Thompson, J. D. (1967). Organizations in Action: Social Science Bases of Administrative Theory. New York: Mc-Graw Hill.

Tuan, Y-F. (1974). Topophilia. Englewood Cliffs, NJ: Prentice-Hall.

Tuan, Y-F. (1977). Space and Place: The Perspective of Experience. Minneapolis, MN: University of Minnesota Press.

Tyrvainen, L., Makinen, K. \& Schipperijn, J. (2007). Tools for mapping social values of urban woodlands and other green areas. Landscape and Urban Planning 79(1): 5-19.

U.S. Department of Agriculture, Forest Service. (2004). Forest Service Social Science Research Agenda. Available at: SSAgenda pages for pdf.p65 (fs.fed.us).

U.S. Department of Agriculture, Forest Service. (2012). National Forest System Land Management Planning Rule. (77 F.R. 21260).

U.S. Department of Agriculture, Forest Service. (1982). National Forest System Land Management Planning Rule. (65 F.R. 67514).

U.S. Department of Agriculture, Forest Service. (2015). USDA Forest Service Strategic Plan: FY 2015-2020. FS-1045. Available at: USDA Forest Service strategic plan: FY 2015-2020 | FRAMES.

U.S. Multiple Use Sustained Yield Act (MUSY) of 1960. (16 U.S.C. §§528-531).

U.S. National Environmental Policy Act (NEPA) of 1969 (42 U.S.C. §§4321-4370).

U.S. National Forest Management Act (NFMA) of 1976. (16 U.S.C. §§472a, 1600-1606). 
U.S. Paperwork Reduction Act (PRA) of 1980. (44 U.S.C. $§ \S 3501-3521$ ).

Vaismoradi, M. \& Snelgrove, S. (2019). Theme in qualitative content analysis and thematic analysis. Forum: Qualitative Social Research 20(3): Article \#23.

Volberda, H. W., van der Weerdt, N., Verwaal, E., Stienstra, M. \& Verdu, A. J. (2012). Contingency fit, institutional fit, and firm performance: a metafit approach to organization-environment relationships. Organization Science 23(4): 1040-1054.

von Bertalanffy, L. (1938). A quantitative theory of organic growth: inquiries on growth laws. Human Biology 10(2): 181-213.

von Bertalanffy, L. (1968). General System Theory: Foundations, Development, Applications. New York: George Braziller, Inc.

von Bertalanffy, L. (1972). The history and status of general systems theory. The Academy of Management Journal 15(4): 407-426.

Wheatley, M. J. (2006). Leadership and the New Science: Discovering Order in a Chaotic World, 3rd Edition. San Francisco, CA: Berrett-Koehler Publishers.

Williams, D. R. \& Stewart, S. I. (1998). Sense of place: an elusive concept that is finding a home in ecosystem management. Journal of Forestry 96(5): 18-23.

Yin, R. K. (2009). Case Study Research: Design and Methods. Thousand Oaks, CA: SAGE Publications, Inc.

Zhang, H., Gao, Y., Hua, Y. \& Liu, K. (2019). Assessing and mapping recreationists' perceived social values for ecosystem services in the Qinling Mountains, China. Ecosystem Services 39: 101006.

Zhang, H., Gao, Y., Hua, Y. \& Liu, K. (2019). Assessing and mapping recreationists' perceived social values for ecosystem services in the Qinling Mountains, China. Ecosystem Services 39: 101006.

Zhao, Q., Li, J., Liu, J., Cuan, Y. \& Zhang, C. (2019). Integrating supply and demand in cultural ecosystem services assessment: a case study of Cuihua mountain (China). Environmental Science and Pollution Research 26(6): 6065-6076. 


\section{FACTORS IN THE USE OF SOCIAL SCIENCE AND SOCIO-SPATIAL DATA IN NATIONAL FOREST PLANNING}

\section{Statement of Need}

The values that people associate with a particular place can influence the land uses people consider appropriate or desirable at that place. Information regarding public values and interactions associated with specific places and landscapes is referred to as socio-spatial data. Socio-spatial data reveals direct connections between people and the biophysical features of specific locations. Tools for collecting socio-spatial data have been available to planners for decades, but it is not clear that they are actually being used in decision-making. This study will identify significant factors that impact how social science and socio-spatial data are integrated into national forest planning.

\section{Study Objectives}

(1) Identify challenges and barriers in integrating social science and socio-spatial data into forest planning processes;

(2) Identify potential avenues for overcoming barriers and facilitating the use of sociospatial data in forest planning.

\section{Methods}

The study includes a review of USFS planning documents and semi-structured interviews with USFS employees in research stations, regional offices and in a sample of national forests.

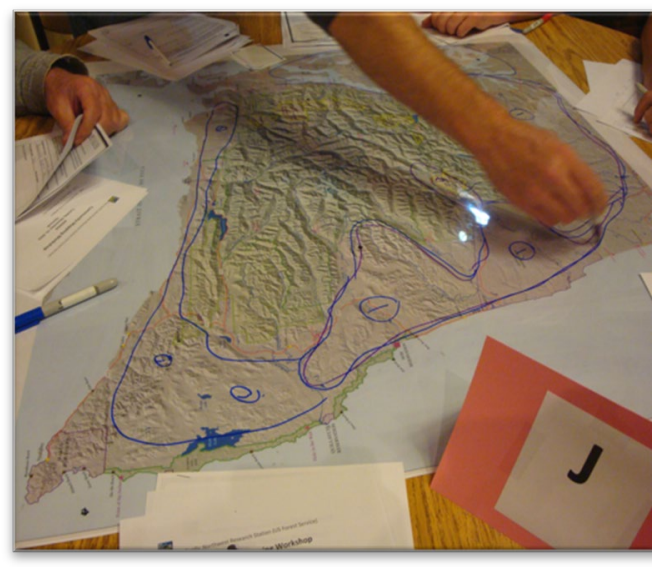

Landscape Values Mapping Workshop on the Olympic Peninsula, WA (2012)
Document Review. A review of a sample of forest plans and related NEPA documents will provide information about what kinds of socio-spatial data have been collected for forest planning and how socio-spatial data have been incorporated into management plans.

Semi-Structured Interviews. About 50 interviews will be conducted with USFS staff and agency and academic researchers to learn about current challenges and perceived opportunities in using social science and socio-spatial data in forest planning. The study targets a sample of forests that have recently completed plans under the 1982 planning rule and those currently planning under the 2012 rule. 


\section{Products}

This study will generate new information about how social science and socio-spatial data are being integrated into national forest planning. The literature review will provide a concise source of information about existing tools and methods for collecting socio-spatial data in natural resource management. A series of technical briefs will summarize the study findings and will (a) identify issues and challenges related to the use of social science and socio-spatial data in forest planning and (b) provide a potential framework for addressing these issues. The study team will also disseminate results to agency leaders through presentations and webinars.

\section{Impact of Study}

The decisions that land management agencies typically make require trade-offs among environmental, social and economic values and objectives. This study directly benefits land management practice by clarifying the existing barriers to and highlighting potential opportunities for the use of socio-spatial data in forest planning.

A better understanding of humanenvironment connections may help land managers identify and minimize potential conflicts, increase trust and cooperation between land management agencies and the public, and reduce time-consuming and costly appeals and litigation of management decisions.

\section{Study Team}

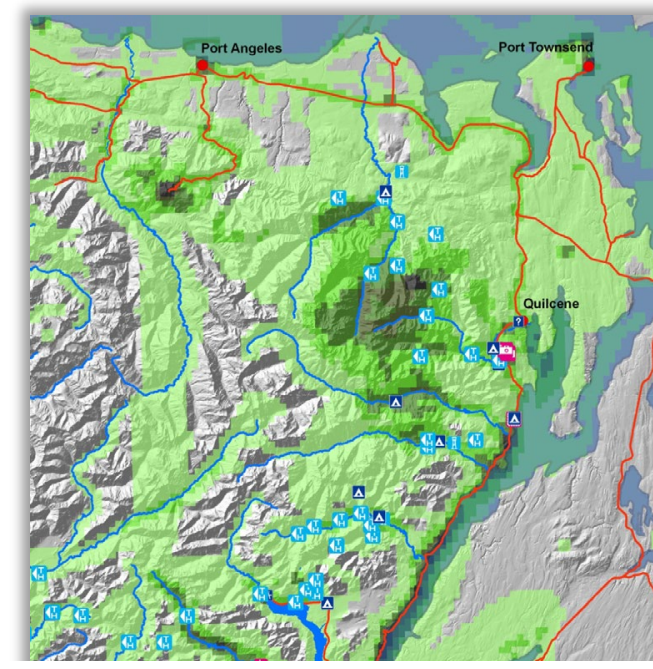

Hotspot map showing valued places in relationship to public access points, Olympic Peninsula, WA (2012)

This research is being funded by the US Forest Service, Pacific Northwest Research Station and conducted as a joint venture agreement (JVA) with Portland State University's Institute for Sustainable Solutions. For further information please contact:

Diane T. Besser, Research Associate Portland State University PO Box 751 - ISS, Portland OR 97207 503-784-7109; dtbesser@pdx.edu
Lee Cerveny, Ph.D., Research Social Scientist USFS Pacific Northwest Research Station 400 N. 34th St., Suite 201, Seattle WA 98103 206-732-7832; lcerveny@fs.fed.us 


\section{Appendix B: Study Consent Form}

\section{Portland State University, Institute for Sustainable Solutions}

Post Office Box 751 - SUST

sustainability@pdx.edu

Portland, OR 97207-0751

1600 SW $4^{\text {th }}$ Ave., Suite 110

http://www.pdx.edu/sustainability

Portland, OR 97201

(503) 725-8556

\section{Informed Consent to Participate in Research - Planners and Practitioners}

Research Project: Factors in the Collection and Use of Socio-Spatial Data in National Forest Planning

As a practitioner or scientist involved in the research, development, or revision of natural resource management plans, we are inviting you to participate in a study by researchers from Portland State University's Institute for Sustainable Solutions and the USDA Forest Service's Pacific Northwest Research Station. We request that you read this form and ask any questions you may have before agreeing to be in the study.

Overview of Study: The study is part of the broad US Forest Service social science research agenda. This study focuses, in particular, on the collection and use of socio-spatial data (information regarding human perceptions about and values toward specific places and landscapes) in forest plan assessment, development and revision. The goal is to identify factors that represent barriers or opportunities in using social science and/or socio-spatial data in order to develop future planning strategies. The full report is expected to be available in March, 2017.

Dr. Rebecca McLain (Institute for Sustainable Solutions, PSU) and Dr. Lee Cerveny (Pacific Northwest Research Station, USFS) are co-leading the study. Diane Besser (Ph.D. Candidate, PSU) is a co-investigator and will be conducting the interviews as part of her dissertation research.

Study Procedures: In the interview you will be asked questions about your work responsibilities and experiences in forest or natural resource planning or research. The only personal questions you will be asked involve your areas of professional expertise and familiarity with social science data and methods. The interview will be conducted during regular business hours. It is expected to last no longer than one hour and can be adjusted depending on your available time. With your permission, we will digitally record the interview in order to more accurately capture your reflections and comments. If you agree to be recorded, you may refuse to answer any question or request that the recorder be turned off at any time. The interviews will take place between Spring, 2015 through September, 2016.

Risks and Confidentiality: The risks associated with this research are minimal. Permissions will be secured from your supervisor, if necessary, to allow you to allocate time during your regular work day to participate. The interview recordings and transcripts will be stored digitally in a secure, password-protected server (for a minimum of 3 years as is standard practice). Data will be available only to the research team and a small sample of the interview transcripts, with all individual identifiers removed, will be shared with an external reviewer as part of the analysis process. Though there is a chance that others in your office will become aware of your 
participation in this study, no individual identifiers will be included in the final report. You may request a copy of your interview transcript to review or amend and you may withdraw your interview narrative from the study at any time.

Your participation in the study is completely voluntary. If you agree to participate, you may decline to answer any question during the interview or withdraw from the study at any time with no risk or harm to you.

Benefits: In sharing your professional knowledge and experiences, you will have the opportunity to contribute toward a better understanding of the barriers and opportunities for integrating social science and sociocultural data into natural resource and forest planning. The information will be used to develop strategies to address the mandates in the 2012 forest planning rule. Lessons learned will also be disseminated through academic journals and technical briefs accessible to a wide variety of planners and practitioners.

Contacts: If you have questions about the study, call Dr. Rebecca McLain at 971-570-3294 or email her at: mclainrj@pdx.edu. For questions about your rights as a research participant, call the PSU Office for Research Integrity at 503-725-2227 or 1-877-480-4400. This Office provides independent oversight of safety and ethical issues related to research involving human participants.

CONSENT: I have received and read the contents of this consent form and have been encouraged to ask questions. I have received satisfactory answers to my questions. I give my consent to participate in this study.

[Oral consent is given for phone or videoconferencing interviews, recorded by researcher.]

Do you agree to have the interview digitally recorded (please initial)? Yes No

Would you like to review your interview transcript (please initial)? Yes No

Would you like to be informed when the report is complete (please initial)? Yes No 


\section{Appendix C: Interview Protocols}

\section{Semi-Structured Interview Protocol USFS Planners and ID Team Leaders}

\section{Informed Consent:}

Before starting the interview, I want to review the informed consent form that I attached to a previous email. I need to make sure that you understand the risks and benefits to you in participating in this study and I'll need to get your consent if you agree to participate.

[Researcher reviews the consent form with the prospective participant, highlighting the research purpose, what will be involved in the interview, the risks and benefits, confidentiality measures, the voluntary nature of participation, and who to contact in case of questions or concerns.]

[Highlight the fact that the interviews will be transcribed and analyzed in aggregate. The transcripts will be kept secured and will not be released to anyone. No individual or national forest will be identified in the final report.]

[Let the participant know that they can change their mind about participating or ask that their interview be removed from the study at any time prior to the release of the final report in March, 2016.]

1. Do you have any questions about the informed consent?

2. If you agree to participate, I will need to record your response. Is it okay to turn on the recorder?

[If they do not wish to be interviewed, tell them you understand their reluctance. Thank the person for their time and ask them if they would like to be informed when the study results are finalized (initial on the consent form).]

[If yes, let the participant know that the recorder will be turned on and ask if they give their informed consent (initial on the consent form). Make sure that the participant knows that they may request that the recorder be turned off at any point during the interview.]

\section{PARTICIPANT BACKGROUND}

GOALS:

- Gather information about participant's areas of expertise.

- Gather information about participant's experience with forest planning.

- Gather information about participant's familiarity with/use of social science and sociospatial data.

\section{QUESTIONS:}

The study I'm conducting focuses on the use of social science and different kinds of geographic or spatial data in forest planning. 
By social sciences, I mean subjects like sociology, geography, anthropology, archaeology, economics, psychology, political science, public administration, public policy, communications.

I have some specific questions that I'll be asking, but feel free to talk about and expand on what you feel is important about this subject. I'll be making some notes about things that I'd like to return to, but, first, I'd like to start by getting to know you better. That will help to guide the rest of the interview.

1. Tell me a little about yourself.

a. What is your current position? What kind of work do you do?

b. How long have you been with this Forest?

c. Tell me a bit about your career trajectory with the Forest Service.

- How long have you been with the Forest Service?

- Where did you get your start?

- What other forests or locations have you worked in?

- What positions have you held?

2. What other kinds of work have you done - either within the Forest Service or other places as you've advanced in your career?

3. I'd like to find out more about your education and training.

a. Tell me a bit about your educational background.

b. What additional training have you received since you have been with the Forest Service that is relevant to your current position?

c. What background or exposure have you had to the social sciences? [Reminder: These include sociology, geography, anthropology, archaeology, economics, public administration and the like.]

d. What is your experience with geographic information systems or GIS?

- What kinds of training have you had in using GIS?

- [If relevant...] Tell me a bit about how you use GIS in your work.

4. [FOREST is in the process or has recently completed a FOREST PLAN]...

a. What stage are you at in the forest planning process?

b. How have you been involved -- directly or indirectly -- in this planning process?

c. Tell me a bit about other forest planning processes you been involved with in the past.

\section{FOREST PLANNING AND THE ROLE OF SCIENCE}

\section{GOALS:}

- Get information about perceived barriers and opportunities in the planning process.

- Get comments on the use of social science and socio-spatial data in forest planning.

- Probe for additional comments about structural, cultural, perceptual/knowledge, and external factors.

\section{QUESTIONS:}

As I mentioned, my study focuses on the role of science, particularly social science, in forest planning. Since you've been quite involved in planning efforts, I'd like to ask you some 
questions about how social science is integrated into the planning process. Let's start with some general questions about interdisciplinary work.

[Since interdisciplinary work is a requirement in forest planning, it is likely that the interviewee will have had some experience working with scientists and specialists with a range of expertise. Probe for comments about organizational structure, culture, external factors and individual perceptions regarding social science data.]

5. Tell me a bit about how you've worked with social science researchers or specialists in any of your projects (these could include geographers, anthropologists, economists, recreation specialists)?

a. What kind of projects? Who did you work with? What was the role of the social scientist (advising, data collection, ID Team)? What prompted the collaboration?

b. Tell me a little about that experience [probe each project/planning effort mentioned].

- What kind of data, information, or science was generated by the project?

- How was the information used in the planning process?

- How was the social science information useful (or not useful)?

- What were some of the benefits of working with social scientists on this project or planning effort?

- What challenges did you run into? [If not noted in challenges, prompt for sufficient resources, funds or time to do the work?]

[Probe for both the relationship with social scientists as well as the type of data collected and its purpose/usefulness.]

6. [If there were specific challenges noted referring to social science in particular], do you think they were unique to your collaboration with social science or have you experienced them in dealing with the biophysical sciences as well?

7. What experiences do you have working with US Forest Service research stations as a source of information or science related to planning? This could include both social science and biophysical science.

a. What information was collected and how was it used?

b. How would you characterize these experiences or relationships? What were the benefits?

c. Describe any barriers that made it difficult to work with the research station. [Probe for barriers regarding both research station administration and working with research scientists.] How did you deal with these barriers?

d. Where did the funding to support the social science research come from [research station, the forest, or other entity, e.g. university]?

e. Did the station respond in a time frame useful to your project or planning effort?

Let's turn now to your current forest planning process and what kinds of social science tools and data you may be using. There are many different kinds, such as recreation planning tools, visitation studies or NVUM data, data from the US census or other socio-economic surveys, historical or cultural data, values or attitudes surveys, or social values mapping.

[Note: Forest sample will target only forests that have, at a minimum, started or completed the assessment process.] 
8. How did your forest go about determining science quality and what constitutes the best available science? Examples might include use of experts or an advisory panel, preparation of a science synthesis.

a. How was that process decided? Who was involved in making that decision?

b. What was the process for determining the best available data from the social sciences I mentioned?

c. What challenges did you run into when assessing data quality? How did you overcome them?

d. If there wasn't a science quality assessment process, why do you think that's the case?

9. The assessment phase in forest planning involves gathering existing scientific data relevant to the forest plan.

a. What kind of social science data did your forest/team gather and/or use in your planning effort?

b. What gaps did you identify in the data? How did you address those gaps? Examples might include recreation planning tools, sense of place or values surveys, socioeconomic analyses, cultural services assessments.

c. How did you use the social science data you collected? What were some of the challenges you encountered in using this data? Examples might include the wrong geographic scale, data that's not specific enough, lack of funds to collect this kind of data.

10. [FORESTS USING 2012 RULE]. The new 2012 forest planning rule has a much stronger emphasis on ecosystem services than in past rules. How do you anticipate the new planning rule will change the kind of data that you need to use in planning? Will there be greater need for any kind of social science data? What might those new data needs be?

11. Socio-spatial data is a special kind of social science data. It refers to data that shows - in map form - what the public values about the landscape and where. I've brought a few examples of this kind of data for you to look at.

a. Have you seen or used this kind of data in any project or planning effort you've been involved in? [If yes...] How has it been used? What were some of the benefits of using this kind of information? What were the biggest barriers in using this kind of information? [If there are barriers mentioned...] What are some of the ways that this data might be made more useful?

b. Are you aware of other efforts to include socio-spatial information in forest planning? [If yes...] Tell me a bit about those efforts. What are/were the successes and challenges in using this kind of information?

c. [For those with no experience with socio-spatial data] I'd like you to reflect a bit on these maps. The dark areas and areas with a high density of dots are places of high sociocultural value.

- How might this kind of data be used in the planning process? [Examples might include determining "need to change" or "desired future condition," assessment to determine gaps in data, facilitating public participation and engagement, addressing cultural services.] 


\section{CONCLUSION}

12. The Chief of the US Forest Service has stated in several speeches that the agency needs more social scientists to address public needs and values.

a. What role should social scientists play in forest management?

b. Where are the greatest needs?

c. What do you feel are the most pressing current forest management problems? How can social science information be more effectively used to address these problems?

13. Do you have any other comments or thoughts that you would like to share before we end the interview?

14. Would you like to have a copy of your interview transcript to review and amend [initial on consent form]?

15. Would you like to be informed when the final report is completed [initial on consent form]?

Thank the participant for their time and let them know that their input is valuable.

\section{Semi-Structured Interview Protocol \\ USFS Social Scientists - Regional Offices}

\section{Informed Consent:}

Before starting the interview, I want to review the informed consent form that I attached to a previous email. I need to make sure that you understand the risks and benefits to you in participating in this study and I'll need to get your consent if you agree to participate.

[Researcher reviews the consent form with the prospective participant, highlighting the research purpose, what will be involved in the interview, the risks and benefits, confidentiality measures, the voluntary nature of participation, and who to contact in case of questions or concerns.]

[Highlight the fact that the interviews will be transcribed and analyzed in aggregate. The transcripts will be kept secured and will not be released to anyone. No individual or national forest will be identified in the final report.]

[Let the participant know that they can change their mind about participating or ask that their interview be removed from the study at any time prior to the release of the final report in March, 2016.]

3. Do you have any questions about the informed consent?

4. If you agree to participate, I will need to record your response. Is it okay to turn on the recorder?

[If they do not wish to be interviewed, tell them you understand their reluctance. Thank the person for their time and ask them if they would like to be informed when the study results are finalized (initial on the consent form).] 
[If yes, let the participant know that the recorder will be turned on and ask if they give their informed consent (initial on the consent form). Make sure that the participant knows that they may request that the recorder be turned off at any point during the interview.]

\section{GOALS}

- Gather information about the challenges/opportunities the participant identifies regarding their work.

- Gather information about how the participant's work has been or is being used in forest planning as well as how he/she would like to see their work used in land management or forest planning.

- Collect input about what needs to happen for socio-cultural and socio-spatial data to become more widely used within forest planning processes.

\section{QUESTIONS:}

As I mentioned a moment ago, my study focuses on the use of social science and spatial data in forest planning. In general, I'll ask you about your past and current work in this area, but feel free to talk about and expand on what you feel is important about this subject. First, I want to get a bit of background to help guide the interview.

16. Tell me a little about yourself.

a. What is your current position? What do you consider to be your area of expertise?

b. Tell me some highlights about your career trajectory with the Forest Service.

- How long have you been with the Forest Service?

- Where did you get your start?

- What positions have you held?

17. I'd like to find out more about your education and training.

a. Tell me a bit about your educational background.

b. What additional training have you received since you have been with the Forest Service that is relevant to your current position?

c. What is your experience with using geographic information systems or GIS?

- What kinds of training have you had in using GIS?

- [If relevant...] Tell me a bit about how you use GIS in your work.

The regional offices have a lot of different functions. I'd like to find out more about what you do in particular so that I can focus my questions.

18. What does a typical day, or perhaps week, look like for you?

a. What do you spend the bulk of your time doing?

b. Is this what you want to be doing?

19. What would you consider to be your most important tasks or goals? [For each task/goal...]

a. Why do you feel these are the most critical tasks?

b. Who do you work with to accomplish that task?

c. What support do you have to do this work? [This could be things like authority granted by your Director, access to other groups within the regional offices or elsewhere, specific funding, etc.]

d. Are there resources or support that you would like, but don't really have right now?

e. What are the biggest challenges that you face in doing your work? 
20. What teams (RLTs) or other groups (HD) have you been (or are currently) active in? [For each team or group...]

a. What is the purpose or goal of that team or group?

b. What is your function within that group? Who are the other members in that group (is this an interdisciplinary group)?

c. [If in an interdisciplinary group] In what ways have you advocated for social science? Where do you see social science as having an impact on the group's tasks?

21. Do you regularly communicate or interface with the other regional offices in other ways?

a. If so, under what circumstances? What are you seeking to accomplish (e.g. professional support, data and information)?

b. If not, why not? Is this something that you would like to do or do you feel it is unnecessary?

22. How do you generally work with or interface with the forests in your region?

a. What kind of information or services or professional assistance to you typically provide for them? In what context (what were they asking for)?

b. How do you assist your forests in their forest planning process (or perhaps at the project level)?

c. Where have you been able to advocate for or integrate social science into forest or project-level planning (e.g. pre-assessment, public participation, assessment, NEPA documents, monitoring)?

- What do you see as the greatest benefits of integrating social science and socio-cultural data into forest or project-level planning?

- What challenges have you encountered in the integration of social science into forest or project-level planning?

23. What experiences do you have working with US Forest Service research stations as a source of information or science related to planning? This could include both social science and biophysical science.

a. What prompted your collaboration? Why did you approach the research station?

b. How would you characterize these experiences or relationships? What were the benefits?

c. Describe any barriers that made it difficult to work with the research station. [Probe for barriers regarding both research station administration and working with other scientists.] How did you deal with these barriers?

d. Did the station respond in a time frame useful to your project or planning effort?

24. I'd like to turn to the issue of socio-spatial data, this is data that attaches human values to particular locations.

a. Have you seen or worked with socio-spatial data or maps that showed what people value about your forests and where?

b. If so, what was the context? What was the data trying to show?

c. How was this data used? At what scale (regional, forest, project-level)?

\section{CONCLUSION}

25. The Chief of the US Forest Service has stated in several speeches that the agency needs more social scientists to address public needs and values. 
a. What role do you think social scientists should play in forest management?

b. Where do you see the greatest needs?

c. What do you feel are the most pressing current forest management problems?

d. How can social science information be more effectively used to address these problems?

26. Do you have any other comments or thoughts that you would like to share before we end the interview?

27. Would you like to have a copy of your interview transcript to review and amend [initial on consent form]?

28. Would you like to be informed when the final report is completed [initial on consent form]?

Thank the participant for their time and let them know that their input is valuable. 


\section{FLATHEAD NATIONAL FOREST (Montana)}

\section{FOREST DESCRIPTION}

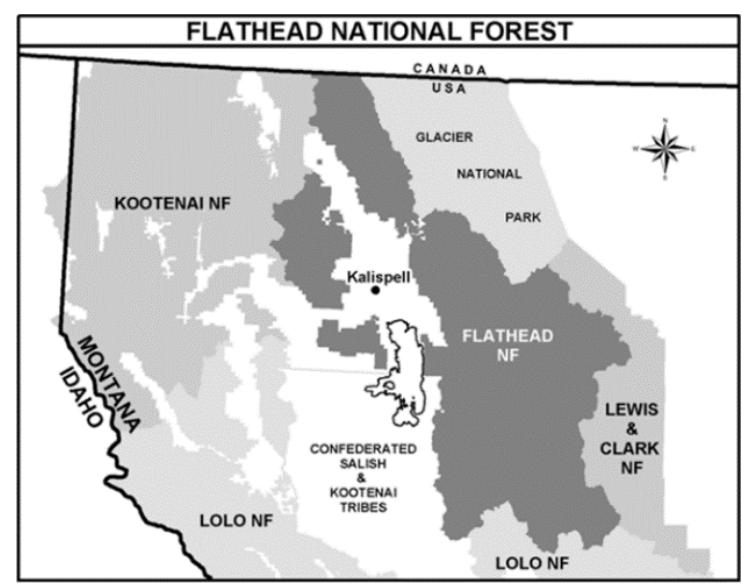

Located in the Rocky Mountains in northwestern Montana, the Flathead National Forest was established in 1897 (as a forest reserve). It extends up to Canada in the north and is bordered by Glacier National Park in the east and three additional national forests (Lewis \& Clark, Lolo and the Kootenai) to the south and west. The Flathead consists of 2.4 million acres, of which approximately 1 million are designated wilderness areas. About 270,000 acres within the forest are nonfederal lands, consisting of privately

owned and state forests. The Flathead National Forest is also the traditional homeland of the Kootenai and Salish (or Flathead) native peoples and, to a lesser extent, the Blackfeet people. The Confederated Salish and Kootenai Tribes of Montana have reserved treaty rights to regional forest resources under the Hellgate Treaty of 1855 . Kalispell is the largest city in the vicinity, with a population of approximately 20,000 persons. However, Flathead County, with a population close to 95,000 , is the fastest growing county in Montana.

The Flathead is quintessentially Rocky Mountain high country, with alpine meadows, towering snow-covered peaks, wild rivers, numerous lakes and wetlands. It is built from block fault mountain ranges carved by glaciers and covered with thick forest. The Flathead and adjacent forests contain critical habitat for protected and endangered species, including the grizzly bear, Canadian lynx, gray wolf, bald eagle, and bull trout, many of which are jointly managed by several federal agencies under governmentmandated conservation strategies. Primary activities in the non-wilderness sections of the forest include timber harvesting and heavy year-round dispersed and developed recreational use (including two ski resorts). Other popular permitted uses include hunting and fishing, berry harvesting (primarily the coveted wild huckleberry), gathering firewood for home heating, mushroom harvesting, and the cutting of Christmas trees. Although the volume of timber harvest has declined over the decades, the industry continues to be important to the local economy. State economic assessments indicate that Flathead County and four adjoining counties derive a higher percentage of their employment from timber-related industries than either the state or the nation as a whole. Jobs in the recreation sector also bring significant revenue into the local economy. Twenty percent of local employment is tied to tourism-related industries. Of note, 
according to visitor surveys, 75 percent of visitors are primarily local residents who are frequent users (about 28 percent of visits are made by people who enter the forest more than 50 times a year).

Management challenges in the Flathead reflect the immense diversity of its resources and users. The forest must (1) address the conservation needs of numerous protected and endangered species (often with very large and/or specialized habitat ranges that extend beyond the forest's boundaries); (2) maintain the quality of and access to a wide range of forest resources and services for local communities; (3) meet timber production quotas while supporting local economies; (4) provide a wide variety of year-round motorized and non-motorized recreational opportunities in developed and backcountry settings; and (5) manage the uncertainties of climate change and its effects on the environment, including an increasing threat from wildfire. Development of the forest's management plan requires the inclusion and input of numerous entities including tribes, federal and state agencies, private landowners, local residents, and a dizzying number of local, regional and national special interest groups.

The Flathead National Forest had been operating under a 1986 forest management plan with 20 extensive amendments, most addressing the needs of species of concern. After an aborted attempt in early 2000, the forest initiated a plan revision process in 2013, under the 2012 planning rule (though not an official early adopter). The formal Notice of Intent was filed in March, 2015, after completing the Assessment Reports and an intense public collaboration effort the previous year. The Draft Plan and DEIS were released in January, 2016, followed by additional public meetings to discuss the proposed alternatives. The Final Plan and FEIS were submitted in November, 2018.

\section{Flathead Plan Revision Timeline}

1. Public Collaborative Process Begins (October 2013)

2. Assessment Reports (April 2014)

3. Public Workshops - Mapping (May 2014)

4. Stakeholder Report (October 2014)

5. Notice of Intent (March 2015)

6. Scoping (March-May 2015)

7. Draft Plan/DEIS (January 2016)

8. Public Input on Draft Plan (2016-2017)

9. Final Plan/FEIS (November 2018)

\section{FOREST PLAN REVISION \& PUBLIC ENGAGEMENT}

Public participation in plan revision: The Flathead has faced numerous appeals and litigation regarding management decisions over the past decades; balancing the desires and demands of many different stakeholders and interest groups is an ongoing challenge. The forest initially contracted with the U.S. Institute for Environmental Conflict Resolution in 2012 to first explore forest staff and stakeholder desire to collaborate on a revised forest management plan and then to set up a process in which to do so effectively. 
Both forest employees and stakeholders indicated a neutral third party would be imperative to a successful collaborative process. The Meridian Institute was selected to implement this process, which began late in 2013. The Institute facilitated numerous topical work groups, set up interagency and tribal groups, arranged monthly public meetings and workshops in communities around the forest and facilitated field trips with the community and forest specialty staff. The Meridian Institute also prepared a written survey focusing on two major issue areas: Habitat-Vegetation-Disturbance and Recreation-Access-Wilderness.

In May, 2014, after release of the forest's Assessment Reports, Meridian held a series of public workshops to assist in determining priorities for the forest's management areas.

Several of these workshops included a mapping component where participants were able to identify specific areas of special interest in various topic areas. In addition, a collaborative mapping tool (Talking Points Collaborative Mapping Tool or TPCM) was initiated which allowed a broader public to also map and add comments about the proposed management areas and, in particular, contribute to the dialogue about recommended wilderness areas. The TPCM is an online interactive PPGIS mapping program developed in partnership with the USGS programming team to facilitate public engagement and collaboration in the forest planning process. The dialogue and recommendations from this public engagement were used to develop the Proposed Action (Need to Change) - released in March, 2015 - that began the official scoping process. The Forest received over 22,000 public comments on the Proposed Action during the 70day comment period that followed. This scoping period, as well as public input the previous year, identified several significant issue areas including (1) vegetation management; (2) timber production; (3) fires and fuels management; (4) wildlife habitat; (5) access and recreation; and (6) recommended wilderness.

\section{HIGHLIGHTS OF THE FLATHEAD PLAN REVISION PROCESS}

The Flathead conducted their plan revision using the new 2012 planning rule, though not an early adopter. From the beginning, regional and forest leadership resolved to complete the plan revision process in five years - a very tight timetable - and succeeded. What led to this success was both organizationally strategic and practical.

Priority status and dedicated ID team. Leadership at the regional office and on the forest agreed, up front, that plan revision was to be the top priority. In doing so, the forest was able to assemble a dedicated planning team, access expertise from diverse inter- and intra-agency sources in a timely manner when requested, and even leverage other budgetary sources by "highjacking" specialists from other programs to assist in completing various subtasks. When asked what advice might be helpful to other forests going through the revision process, one team member commented:

I would organize with a full-time team and make it the forests \#1 team. That needs to be the sole focus and you need to get in, get out. Otherwise it becomes protracted and nobody's a winner in these long-term planning efforts. By the time you're 7-8 years in, some of the data you started with is now stale. What are 
you going to do? Start over? It's really important to get in and get out and to recognize that you're not going to have all the answers.

We're going to get some things wrong! As a practical matter, forest leadership recognized that plan revision is an imperfect process, especially when dealing with an expansive forest with a myriad of management challenges and looking at that landscape from a 30,000 foot viewpoint. Leadership acknowledged that certainty in predicting what management actions will result in what outcomes is an unrealistic, if not impossible, goal. They felt that shifting from thinking of a forest plan as a 'finished' document to seeing it as a 'working' document could prevent the unending revising and delays that happen when team members are just 'waiting for that last bit of data.' And, perhaps, such a perspective might allow for more honest, transparent and realistic communication with the public.

\section{SOCIO-CULTURAL AND SOCIO-SPATIAL DATA IN PLAN REVISION}

Social science, socio-spatial data and the assessment report: The socio-economic and cultural sections of the Flathead's Assessment Report contain the typical tables drawn from U.S. Census, Department of Labor, Department of Commerce, and various state and USFS databases. The data is generally coarse - reported by county (the Forest is situated primarily in Flathead county but also spans 3 adjacent counties). A large proportion of tables and figures relate to timber harvest, fire and fuels management, and agriculture. A smaller proportion deal with recreation and public access. Much of the recreation and roads plan information is derived from INFRA as well as NVUM. Sections dealing with socio-cultural services and historical uses note several key public values regarding forest benefits and services. Values such as solitude and spiritual experience were specifically named as especially important, though the data source is not cited. ROS/WOS, a Recreation Facilities Analysis (from a report completed in 2007), SMS, and special permit data were used to assess recreation infrastructure and services. The report does not specifically display or cite data - socio-spatial or otherwise - collected from public workshops, the TPCM application, or any public survey conducted by the Meridian Institute. However, the Institute's Stakeholder Report notes that the primary goal in the public engagement process was to allow stakeholder's to come together to reach common ground on the key management issues and priorities in the spirit of collaborative problem-solving, not to collect 'best available science' for the Assessment Report.

Public attitudes and values: The social and economic sections of the Assessment Report strongly emphasize that the culture and lifestyles within the four-county Flathead area are tied strongly to the geography and rich natural resources in the region. It refers to lifestyles that are based in great part on the outdoors. Of note, the introduction to the Assessment Report states that it would have benefited from primary assessment "which would include information about how people define and explain their ties and connections to the land, how they explain the issues of importance... from their own point of view. This assessment would have benefitted from an assessment similar to those done utilizing an ethnographic method for the Kootenai National Forest, the Idaho Panhandle National Forest and the Nez Perce and Clearwater National Forests" (Flathead 
Assessment Report, 2014, p. 4). These ethnographic studies stress the importance of people's relationship to the land, the benefits and services the forest provides, and the associated values attached to these resources.

\section{DISCUSSION: The Spectrum of Socio-Spatial Data}

During the plan revision process at the Flathead, socio-spatial data showed up in planning documents and public workshops in a multitude of formats, informing the planning process both explicitly and implicitly. Maps were used in workshops to engage the public in discussions around boundaries of management areas, talking about management challenges with water and species conservation, assessing roadless areas or recreation zoning, and numerous other topics. The online TPCM was used to both inform the public through the display of various layers associated with the draft plan and gathering public comments on proposed alternatives, particularly with regard to designated wilderness areas. All proved useful in informing planning decisions:

For the Flathead, the wide spectrum of socio-spatial data can proved useful, from simply naming special places in a survey to direct interaction with GIS layers in an online application. The extent to which this socio-spatial data is integrated into planning documents depends on several factors including (1) the availability of the data at key moments in the process (assessment, public engagement, document preparation); (2) the expertise and/or capacity of staff to interpret and integrate the data; (3) time within the schedule for any data collection activity; and (4) the resources or budget needed to collect, process, analyze and integrate socio-spatial data. 


\section{Appendix E: Colville National Forest Brief}

\section{COLVILLE NATIONAL FOREST (Washington)}

\section{FOREST DESCRIPTION}

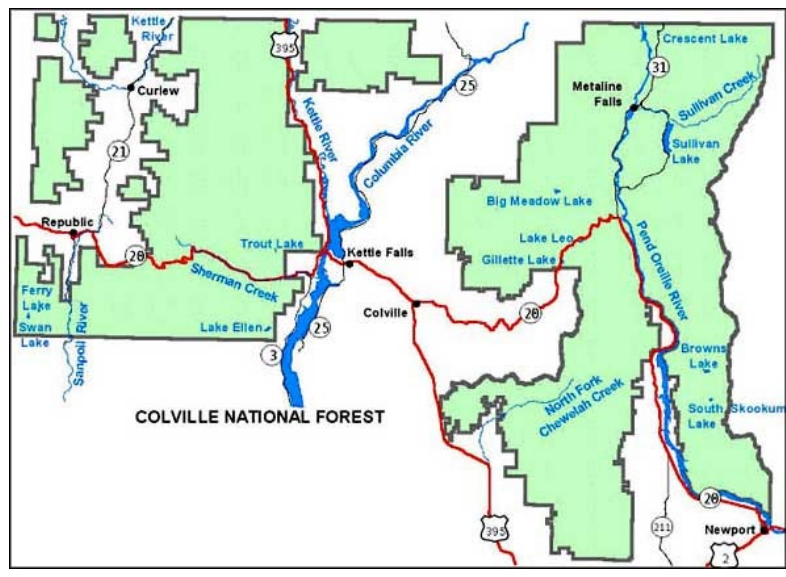

The Colville National Forest is located in the far northeastern corner of Washington State, adjacent to the Canadian border to the north and Idaho to the east. Officially established in 1907, the Colville encompasses 1.1 million acres nestled in the western foothills of the Rocky Mountains and straddling the upper reaches of the Columbia River. This part of the state is sparsely populated. The community of Colville, with a population of about 5,000 persons, is the county seat of Stevens County. The larger 3-county "economic zone" in which the Forest is situated contains an additional 30,000 people living in smaller, dispersed communities. The city of Spokane, WA, lies 65 miles to the south. Other populated areas include the Kalispel Native Community of the Kalispel Reservation, the Confederated Tribes of the Colville Reservation, and the Spokane Tribe of the Spokane Reservation.

The Colville National Forest is part of the ecologically diverse Columbia Highlands, situated in between the Cascades and Rocky Mountains. The Colville and large swaths of adjacent state and privately owned forest land contain dryland forests of ponderosa pine and Douglas fir, species that thrive in the wetter areas, such as western hemlock, red cedar, and white pine, and significant areas of western larch and lodgepole pine. Not surprisingly, timber production has been (and still is) an important activity in these forests. Major employers in the area include Boise Cascade, Stimson Lumber, and Vaagen Brothers Lumber (as well as the US Forest Service and the Washington State Department of Natural Resources). The Colville supports a wide variety of flora and fauna, including the only remaining herd of woodland caribou in the lower 48 states and a large population of Canadian lynx, considered a threatened species under the Endangered Species Act. It also contains over 200,000 acres of old-growth forests, a critical habitat for the endangered spotted owl. Regulations regarding protection of these old-growth forests fall under the regionally-focused Northwest Forest Plan of 1994.

Despite its remote location, the Colville attracts a large number of visitors each year and provides an impressive variety of year-round provisioning services and recreational opportunities (including a downhill ski resort). Hundreds of miles of forest roads and trails provide access to both developed and backcountry areas that are popular for 
gathering forest products such as mushrooms and huckleberries, nature viewing, hunting and fishing, hiking, camping, white water rafting, mountain biking, rock climbing, crosscountry skiing, horseback riding, snowmobiling and OHV use. The Forest is also the homeland of the Kalispel people, who have relied on its plentiful bounty for thousands of years. Many Native American tribes gathered annually in the area to fish and trade in numerous commodities from as far away as central Montana and the Pacific coast. A rich spiritual tradition is interwoven with these activities that continues today. The First Salmon Ceremony is still celebrated at an intertribal pow-wow at Kettle Falls each year.

Management of the Colville has often been contentious, due to the diversity of landscapes, resources, and private and governmental interests at play. Land allocations have been a particularly contentious topic. Only one percent of the Forest had been designated wilderness as the current forest plan revision process commenced, which spurred a strong push from conservationists to consider portions of the inventoried roadless areas for this special status. This has often elicited a counter response from both recreationists and the timber industry. Tempering the disputes regarding management of the Forest has largely been achieved through adoption of a highly collaborative, inclusive, and ongoing public engagement process, nurtured for close to two decades.

At the start of the plan revision process, the Colville National Forest had been operating under a 1989 forest management plan which had been amended 40 times. Aborted plan revision attempts occurred in 2002 and 2006 under updated planning rules that were subsequently invalidated in legal challenges. A new Notice of Intent to Revise the Forest Management Plan was filed in June, 2011, in concert with the Okanogan-Wenatchee National Forests, after court rulings allowed forests to revert back to the 1982 planning rule. The Colville separated its plan revision efforts from the Okanogan-Wenatchee in 2014. The intense public engagement process that began in 2004, as part of previous plan revision attempts, continued in earnest through 2017. The Forest's draft plan and DEIS were released in February, 2016, followed by an extended public review period. The Final Plan, FEIS and ROD were released in October, 2019.

Colville Plan Revision Timeline (1982 Forest Planning Rule)

1. Public Workshops (2004-2008) - previous plan revision attempts

2. Collaborative Engagement (2007-2015)

3. Notice of Intent/Proposed Action (June 2011)

4. Colville plan revision split from Okanogan-Wenatchee (2014)

5. Draft Plan/DEIS (February 2016)

6. Public information sessions and webinars (2016-2017)

7. Final Plan/FEIS \& ROD (October 2019)

\section{FOREST PLAN REVISION \& PUBLIC ENGAGEMENT}

Public participation in past plan revision efforts: Public engagement in the Colville's plan revision process began back in 2004 with a series of public workshops and 
continued for a few years as part of initial attempts to revise the 1989 management plan. At the time, the process was initiated jointly with the Okanogan-Wenatchee National Forest to the west. Workshops were held in a dozen different communities spread throughout the region (Yakima, CleElum, North Bend, Wenatchee, Winthrop, Okanogan, Tonasket, Republic, Colville, Ione, Newport, and Spokane). Additional public meetings took place in the ensuing years, despite the fact that the formal revision process itself was stalled and often mired in controversy. After review of forest-specific public comments and resource issues, the Colville and Okanogan-Wenatchee revision processes separated in 2014.

The Northeast Washington Forestry Coalition (NEWFC): Established in 2002 to address the gridlock surrounding management on the Colville and Okanogan-Wenatchee National Forests, the NEWFC created a working group of timber companies, conservationists, business owners, forestry professionals, and recreation groups. In early 2006, the Coalition completed a draft "blueprint" collaborative management proposal for the Colville National Forest after a lengthy multi-stakeholder engagement process involving coalition members as well as federal and state agencies and tribal interests. Additional refining of the proposal took place in a year-long USFS-led "summit" consisting of a series of workshops attended by coalition members, county commissioners, and interested local residents (March, 2006 through January, 2007).

In early 2009, a Washington senator and representative co-hosted a forum in Spokane to discuss the success of the NEWFC and address still unresolved issues associated with management of the Forest. A new collaborative, named the Roundtable, was initiated that established four issue committees: Mining, Recreation, Ranching and Tribal Issues. These committees were tasked with reaching agreements about inventoried roadless area management and, in particular, new wilderness areas (one of the pressing and more contentious forest management challenges). In 2010, Conservation Northwest (one of the NEWFC members), through its Columbia Highlands Initiative, facilitated drafting of a legislative proposal focused on recommended land allocations.

Incorporating lessons learned from the Roundtable discussions and the legislative proposal put forth by Conservation Northwest, the updated NEWFC blueprint proposed allocations for active forest management, forest restoration, new designated wilderness, a new conservation area and three new recreation areas. The Coalition's blueprint proposal was submitted to the Colville's Forest Planning Revision Team and became an important working document in the ongoing plan revision process.

Of note, in 2007, during this critical collaborative phase, the Colville was selected as one of three national forests to practice a new USFS business model that focused heavily on collaboration with the public and special interests in forest management planning and projects. The foundational concept of the model suggests that close and early collaboration with public interests combined with a predictable budget cycle to support these activities can improve a Forest's relationship with communities, increase public involvement in and acceptance of forest management decisions, and ultimately decrease 
overall operational costs. The Colville reports that the collaborative model has been highly effective not only in the ongoing plan revision process, but also at the projectlevel. For example, the Forest has moved 22 timber-related projects forward without appeal or litigation.

Public participation in the current plan revision process: In 2014, the Colville separated from the Okanogan-Wenatchee and continued its plan revision process. Engagement with the public, interest groups and the NEWFC, culminating in the release of the Colville Draft Plan and DEIS in February, 2016. After the draft plan was released, forest staff gave presentations at numerous forums and produced several webinars during the extended public comment period that followed. Webinars addressed key issues of public concern and management challenges including (1) Forest Access by Roads; (2) Forested Vegetation Management; and (3) Socio-Economic Concerns. Numerous open houses and listening sessions were also arranged. Much of the public outreach was designed and facilitated by an outside contractor, the U.S. Institute of Environmental Conflict Resolution, and a sub-contractor, Enviro. Input into the draft plan occurred throughout 2016 and 2017, a long period of public input in which formal and informal comments were considered and proposed alternatives adjusted to reflect public sentiment. The Final Draft and FEIS were entered into the record in October, 2019.

\section{HIGHLIGHTS OF THE COLVILLE PLAN REVISION PROCESS}

Constructing the final plan under the 1982 planning rule with the vision of the 2012 planning rule: While technically the Colville's Final Plan conformed to the 1982 planning rule, the vision framing the process aligned with the 2012 planning rule. Each chapter in the final plan starts with a Desired Conditions statement aligned with the 2012 rule followed by standards and guidelines typical of a plan developed under the 1982 rule. As one planning team member explained:

....under the 2012 rule, they're going to have to shift their thinking...because I think in reading the 2012 rule, it says that when you're doing projects on a forest, what you really need to be concerned about is -- are you moving the forest toward a desired condition or not? It's not about compliance with a standard and guide...I'm in compliance with standard number 42 of the forest plan, and that's it... what you need to think about and the place you need to look is the desired conditions description....and that's what we started writing was the desired conditions.

Collaboration and public outreach: The Colville implemented an expansive public outreach effort during the long plan revision process involving the public, special interest groups, the NEWFC collaborative, advisory councils (FACA-endorsed), and government and tribal officials. ID Team members indicated that the success of this effort in both informing the final plan and engendering public acceptance of it was due, in large part, to contracting with external facilitators. This strategy helped to insert the skillsets needed for facilitation and public outreach that at times were not available internally (primarily 
due to staff turnover) and lessened the burden on technical and specialist staff to design public sessions, prepare materials, compile debrief reports, etc.

Well, kind of looking at it hindsight, 20/20, what's benefited us in this last round is really understanding that there's going to be those folks who want to know everything about an inch deep, right? And so we've had some help from our contractor in helping frame things...you might call it the Readers' Digest version or the executive summary...And then we know we've got a certain percentage of our audience that are more technically astute... They want to dig deep into your references for the science that the specialists use with their reports...we're trying to find that happy medium where the key things that we can provide that information, we'll just post it on the website or make it available, in addition to this sort of Readers' Digest highlights, key points kind of process.

Leadership and the business plan: Navigating the fits and starts that characterized the Colville plan revision process was made much smoother by the initial establishment of a "business plan" that laid out the operational mechanics of the process and who would make what decisions. This was key in maintaining forward motion even given the discord and workflow disturbance that can often result with multiple staff turnovers during a long plan revision effort.

I would say that another thing we did right at the beginning when I started was we sat down and we wrote a business plan, and part of that business plan was very specific about who in the regional office could formally tell the forest plan revision team what to do... that was the regional forester and the director of planning. Nobody else.

\section{SOCIO-CULTURAL AND SOCIO-SPATIAL DATA IN PLAN REVISION}

The utility of socio-spatial data: The Colville used maps prolifically in their public outreach which included an interactive GIS-based mapping webpage displaying layers on various issue areas, such as recreation, wilderness, etc. In several public information sessions, facilitators placed maps on the walls where the public could indicate their recreational values and use preferences by drawing on these maps. As the quote below indicates, ID team members found the data produced by these exercises lacking the robustness needed for the more formal analyses that go into creating a comprehensive management plan. However, the conversations that occurred and insights that were gleaned during these types of activities did indirectly influence ID Team discussions about what the public values and decisions made about formulating Draft Alternatives.

Well, taking the recreation one where we asked people to identify their special places, I think that was a hit and a miss with that, maybe in just the way we did it. We had maps on the wall and we said, "Just draw a circle around what your favorite place is and tell us what recreational activity you do in that place." And people either drew great big huge circles or they did a dot. So the usefulness, sort 
of like the rigor of that information, may not have been really great. But...there was a motorized recreation group and a very strong non-motorized recreation group looking at the maps...they both pretty much circled the same area...it came out that both of them enjoyed the sense of solitude that they got...And they looked at each other..."Oh, we have something in common, don't we?"...hearing that conversation about solitude, that's when we [the ID team] came back and we did have discussions about... where maybe we need to look at these roadless areas and see if we can put in some motorized recreation.

Forest planning is NOT the place to do beta testing. One particular comment highlights the often conflicting agendas and goals of planning and research. This comment has both explicit and implicit points. Certainly, a forest deep into the plan revision process does not have the time or capacity to accommodate a research agenda. But it also indicates that the stages in the process, as historically practiced, may not be conducive to the collection of extensive qualitative values-based socio-spatial data. This is evident in the placement of public mapping exercises in the Colville revision strategy.

What I experienced were various researchers calling me up, saying, "Do I have a deal for you! I have this model that will do X. We just feed in these variables and this sort of data, and it'll just spit out all the answers in the end that you're looking for"....after a while, I figured out that I needed to ask questions like, "Have you ever run this model before on any forest?" And the answer nine times out of 10, "No, but I can run it on yours"...And up front, I had decided that we were not going to be anybody's beta testers because we didn't have the time to do that. And we were not going to take the risk of investing in some model that's going to take a year to gather the data or whatever and then it's not going to work.

Is it the sole responsibility of the USFS to collect socio-spatial data? Members of the ID Team seemed to understand the value of socio-cultural data (and ostensibly sociospatial data). They often rued about the lack of more localized and timely social, cultural and economic data that would help them more directly identify and address the needs particularly of the surrounding communities. Forest assessment reports that informed the draft plan often depended on economic analyses at the county level, at best, recreation surveys (e.g. NVUM) that were outdated, and incorporated little to no information about informal uses of the forest (such as mushroom or berry harvesting). However, there was uncertainty about how to gather such data or even whether it really was the sole responsibility of the USFS to do so given the complexity and inter-dependencies inherent in natural resource management.

I would say that there's high interest in that kind of information [socio-spatial data] from county commissioners, from some people in the community, business people. They're wrestling with it [localized issues] every day...I don't know if the social, more of a recreation trends and some of those other social things, some of those other drivers of why things are not great economically, it can't be, in my 
own opinion, it can't be all on the shoulders of the federal lands. I mean, that's a piece of the puzzle, but there's so many other drivers as to why things are shifting away from certain things...climate change and all.

\section{CONCLUSION}

As is evident in the three sections above, staff at the Coconino recognize the value of socio-spatial data, but the utilitarian nature of such data is questioned within the context of the plan revision process. Typically, stage one in the process begins with a public notice of intent followed by a period of compiling bio-regional and forest assessments and perhaps a science synthesis. This is certainly an inter-disciplinary effort among natural and social scientists in the regional offices and research stations as well as the resource specialists at the forest and it may include a formal stakeholder analysis.

Scoping and soliciting public input can occur during this stage depending on the forest's public engagement plan and plan revision schedule, but it is difficult at this stage to communicate to the public how their input will be incorporated into a draft plan (that is often 1-2 years away from completion). In addition, the 2012 planning rule, which does emphasize robust and inclusive public participation in the plan revision process, repeatedly includes data-centered language that sees the initial assessment phase as the "rapid evaluation of existing information about relevant ecological, economic, and social conditions, trends, and sustainability and their relationship to the land management plan within the context of the broader landscape" (36 CFR, Part 219, Subpart A, $\$ 219.5(\mathrm{a})(1)$, emphasis added). This provides little incentive to use the assessment phase to gather new information targeting public values and use preferences, particularly if staff capacity and/or budgets are limited.

It may be necessary to reassess the function of socio-spatial data within the context of forest planning and where it might best fit. Is it a data collection tool that belongs in the assessment phase, where new data are collected that directly inform the draft plan? Or, is it a tool to determine the viability or acceptability of the various alternatives in the draft plan as proposed to the public? Data are tools to achieve a desired outcome and the tool must fit the purpose to be effective. 


\section{Appendix F: Coconino National Forest Brief}

\section{COCONINO NATIONAL FOREST (Arizona)}

\section{FOREST DESCRIPTION}

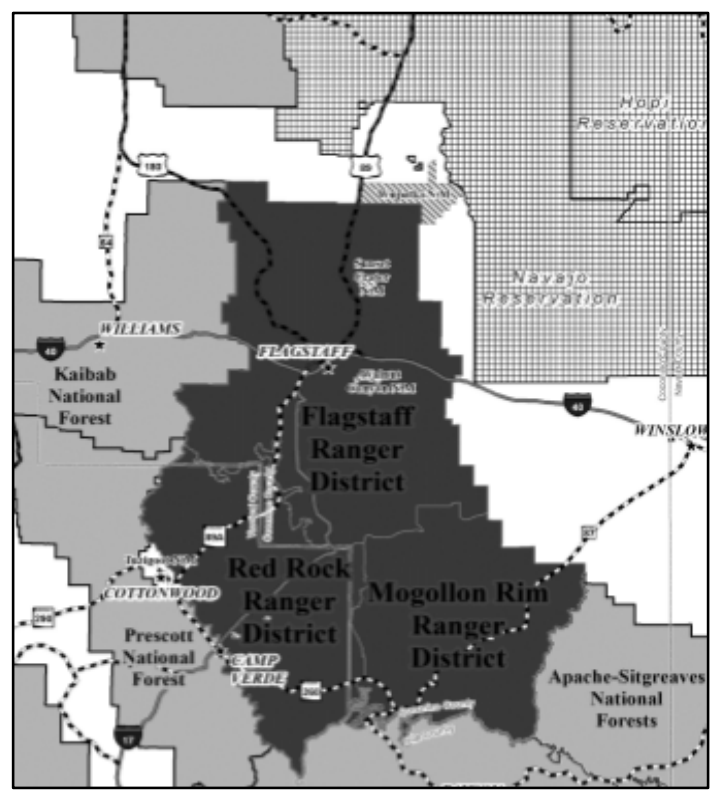

The Coconino National Forest was established in 1908 by President Theodore Roosevelt. The Forest is located in northcentral Arizona, just south of the Colorado Plateau, and encompasses approximately 1.85 million acres. It is bordered by four other national forests and is adjacent to the Navajo and Hopi Reservations. The Coconino contains almost all major biotic communities due to its wide range of topography and elevation (from 2,600 to 12,633 feet) with a high diversity of flora and fauna. The Forest has all or part of ten wilderness areas, Arizona's only wild and scenic river designations and largest natural lake. Several archaeological and cultural sites provide public access to and education about the long and vibrant history of Native

American and Hispanic presence in the area, including prehistoric petroglyphs. Flagstaff, a popular tourist destination, sits in the northern part of the Forest. Current population estimates for the Flagstaff metropolitan area exceed 140,000 persons. The small city of Sedona, southwest of Flagstaff, has emerged as a vacationer's "paradise" due to its numerous natural treasures, spa resorts, and dynamic arts community.

Much of Coconino National Forest is a high altitude plateau with the largest contiguous ponderosa pine forest in North America. Approximately 35\% of the total land area has been historically used for timber production. Recreational use of the forest has a long history as well, spanning over 100 years thanks to early and easy access to the area via transcontinental railways. More recently, population growth in the urban areas and increased tourism has shifted management priorities from traditional commodity production (e.g. timber and mineral extraction) to a much stronger emphasis on day-use recreation services. The Analysis of the Management Situation (i.e. Need to Change) notes that close to 4 million people visited the Forest in 2005, a $72 \%$ increase in only 5 years. Current challenges include increased demand for developed and more diverse year-round recreational opportunities that have often resulted in use conflicts, such as between snowmobilers and cross-country skiers. 
An additional challenge is that the Coconino has one of the highest natural fire occurrences in the U.S. The lack of significant rainfall, high temperatures and strong winds during the summer result in very high or extreme wildfire danger. The potential damage inflicted by wildfire is increased by the fact that there are a number of cities completely surrounded by forest land. To manage fire risk, the Coconino has increased its use of prescribed burns and strategic thinning, particularly in the wildland-urban interface. Unfortunately, these management strategies have also reduced the availability of other forest products highly valued by local communities, such as firewood for home heating, as well as concerns about air quality during fire events.

At the beginning of the plan revision process in 2006, the Coconino National Forest had been operating under a 1987 forest management plan with numerous major amendments. The Forest submitted its Notice of Intent in May, 2010, under the original 1982 planning rule, after making several revision attempts under now defunct rules (2005 and 2008). Forest Assessment Reports were compiled throughout the initial plan revision process from a public survey in 2006 through reports released in 2013. The Draft Plan and DEIS were released in November, 2013. A proposed Final Plan was released for public review in May, 2016 with the Final Plan and FEIS release in March, 2018. Since that time, two amendments have been registered in response to appeal resolutions.

Coconino Plan Revision Timeline (1982 Planning Rule)

1. Assessment Reports

- Public Attitudes \& Values Survey (February 2006)

- Sustainability (March 2008)

- Recreation \& Scenery (July 2011)

- Socio-economic (February 2013)

- Heritage (September 2013)

2. Notice of Intent to Revise Forest Management Plan (May 2010)

3. Scoping and Public Outreach (2008 and 2011)

4. Draft Plan/DEIS (October 2013)

5. Final Plan/FEIS and Record of Decision (March 2018)

6. Amendments (June \& November 2019)

\section{FOREST PLAN REVISION \& PUBLIC ENGAGEMENT}

Public Participation in Plan Revision: Public input in the Coconino plan revision process began in 2006 (in a previous revision attempt). Additional targeted stakeholder meetings were held in 2008 and again in 2011 to complete an Analysis of the Management Situation in the current revision process (also referred to as a Need for Change notice). During the comment period, after the release of the draft plan in January 2014, the Forest hosted seven public workshops and numerous internal meetings with other agencies, local governments, and tribes. The purposes of the meetings were to educate the public about the planning process, inform them of the content of the Draft 
Plan and DEIS, and to share tips for reviewing and commenting on the documents. The meetings were also used to assess what issues were most important to the public. Based on feedback, additional meetings focused on dispersed recreation, forest wildlife and water resources, motorized recreation, wilderness and special areas, and formally permitted forest uses.

Geographic Scope of Public Participation: The Coconino, as is the case in many national forests, has struggled with reaching out to the numerous constituencies and stakeholder groups interested in forest issues, but located over a vast geographic territory. The DEIS notes a total of 92 meetings related to plan revision, from 2006 through 2013 25 of the meetings were public open houses, forums and information sessions. Public meetings were hosted in communities within and surrounding the Forest including Flagstaff, Sedona, Happy Jack, Winslow, Camp Verde, Cottonwood and the large city of Phoenix to the south. Of note, the Forest planning team made a concerted effort to schedule targeted presentations and discussions with numerous local interests and "friends of" groups as well as over a dozen meetings with Navajo and Hopi tribal groups. According to the documents, the Forest felt it needed a concerted outreach effort to respond to numerous and diverse local concerns. A major issue important to the public concerned day-use recreationists seeking more "developed" recreational experiences and concomitant devaluation of strategies to maintain local access to "special" areas.

Scoping and review of draft and final plans: A key strategy at the Coconino was to submit proposed draft and final plans for a lengthy public review and commenting period. While this certainly delayed submission of a final plan, staff on the ID Team felt that numerous pre-releases helped to disseminate a consistent stream of information to the public and other interest groups to keep them engaged in the process. It validated and made visible the impacts of stakeholder input on the draft alternatives. Monthly news briefs were able to make connections and show how public comments and other input was incorporated into the draft plan as forest staff worked through preparation and submission of the final plan. This prevented the "black hole" that often occurs between selection of an alternative proposal from the draft plan and the long process of preparing the final plan and environmental impact statement.

\section{SOCIO-CULTURAL AND SOCIO-SPATIAL DATA IN PLAN REVISION}

Social science, socio-cultural data and the assessment reports: While many forests utilize outside contractors, TEAMS experts or staff at regional offices to assist in compiling socio-cultural and economic assessment reports for plan revision, Coconino's ID team had two social scientists (who ultimately moved to other positions at various stages in the planning process) and an archaeologist (responsible for managing 10,000+ archeological sites identified within the forest). The Coconino plan revision webpage contains links to numerous assessments and special reports compiled between 2008 and 2013. Those focusing on socio-cultural and economic data are (1) Socio-Economic Sustainability, (2) Recreation, (3) Scenery, (4) Socio-Economic Assessment, and (5) Heritage. These reports rely heavily on typical secondary data sources from the US 
Census and other federal agencies as well as Forest Service databases, including INFRA, NVUM and the NSRE. Little data is utilized from state or county agencies. Additional assessment tools include the SMS and ROS. Tribal consultations supplemented the Heritage report.

Public values and attitudes survey: A survey was conducted in January, 2006, on the values and attitudes of the public toward the Coconino National Forest (as well as a related survey focusing on Arizona Native Communities). This project used interviews combined with focus groups to identify issues, concerns, beliefs, and values regarding a proposed revision of the 1987 Forest Plan (during one of early plan revision attempts under subsequently invalidated planning rules). Both open-ended and targeted questions were used. The information was placed in the larger northern Arizona socio-economic context. Participants included community members, county and state officials, other government agencies, industry and special interest groups, and tribal members. Emerging themes revolved around perceived population and use pressures on forest resources, preservation of its unique scenic qualities, and deteriorating communityagency relationships.

Both the Scenery and the Socio-Economic Sustainability reports reference the 2006 values survey. The Sustainability report contains a section with a synopsis of the survey results, identifying public values and attitudes directly related to forest resources and socio-cultural sustainability. The Scenery report, interestingly, uses the survey results as the data source for a constituent analysis. Constituent analysis allows for incorporation of public perceptions of attractiveness, identifies special places, and helps to operationalize the meaning people give to the landscape. The constituent analysis for the Coconino involved the following: (1) reviewing and incorporating key management directions from the Sedona-Oak Creek and Flagstaff-Lake Mary Ecosystem Analyses (developed through extensive stakeholder involvement); (2) reviewing requests for special area designations made by the public; and (3) reviewing SMS inventories (particularly the proposed scenic integrity objectives identified during public meetings). Employing constituent analysis leads to a determination of the relative importance of factors such as aesthetics and specialness for particular places. This importance is expressed as a "concern level." Sites, travel ways, special places and other designated areas are assigned a concern level value of 1, 2, or 3 to reflect high, medium, or low importance. The concern level can be used as a variable in the SMS modelling tool.

Landscape Values Mapping on the Coconino: Greg Brown and Patrick Reed (2009; 2012a; 2012b) developed a PPGIS process for national forest planning referred to as landscape values mapping. The Coconino National Forest was one of their pilot studies. The primary purpose of the Coconino project (2006-07) was to test a methodology, and secondarily as a tool to gather socio-spatial data for the Coconino's forest plan revision process. The project transformed traditional landscape values mapping, piloted in the Chugach National Forest in 2000, into what the authors call Values Compatibility Analysis (VCA). VCA is designed to provide quantitative data about how public values mesh with forest management strategies and where biophysical and social values may be 
linked or in conflict. Brown and Reed also introduce the concept of landscape metrics that quantify human perceptions of place using a language and framework borrowed from landscape ecology (e.g. Fragstat). VCA results indicate patches of hotspots as well as a series of calculated metrics that display and describe the distribution of public value characteristics across geographic space, including sum, percent, dominance, frequency, conflict potential, and diversity. VCA still requires collection of primary socio-spatial data on public attitudes and values, as these spatially-referenced base layers are necessary for the analysis and do not usually exist for most forests. As a result, acceptance of the tool by forest planners has been hindered by a lack of good spatial inventories of human attitudes and values as well as organizational challenges, such as the need to obtain OMB approval for data collection of this type (e.g. surveys). There are no references regarding the Coconino VCA analysis in the assessment reports, Draft or Final Plan or DEIS/FEIS.

Brown, G. \& P. Reed (2009). Public participation GIS: a new method for national forest planning. Forest Science 55(2): 166-182 (Deschutes/Ochoco, Mt. Hood, Coconino).

Brown, G. \& P. Reed (2012a). Social landscape metrics: measures for understanding place values from public participation geographic information systems (PPGIS). Landscape Research 37(1): 73-90 (Deschutes/Ochoco, Mt. Hood, Coconino).

Brown, G. \& P. Reed (2012b). Values compatibility analysis: using public participation geographic information systems (PPGIS) for decision support in national forest management. Applied Spatial Analysis \& Policy 5(4): 317-322 (Mt. Hood NF).

\section{DISCUSSION}

\section{Does Landscape Values Mapping and/or other socio-spatial data add value to the} draft or final plan? This question can be answered with a resounding - maybe! In a scan of the assessment documents, clearly the values and attitude surveys were of great value. Both were spatialized (where "special places" were identified) and incorporated into the constituent and the scenery management system (SMS) analyses. However, the values compatibility analysis (VCA) conducted by Brown and Reed was not significantly incorporated into any planning decisions. As one staff member indicated:

I knew about it [the Coconino VCA]. I had a change in staff. A social science person on our revision team ended up changing jobs shortly after I got there. I remember that was one of the things she handed to me saying, "Here, this is something that was done." I think my struggle, and our struggle, was it didn't show anything we didn't already know. It was kind of a color coding of places on the forest that people valued. I could point at each one of those shaded area like 'Yup, that's this canyon. Yup, that's the peaks. Yup that's...you know." So it was more confirming of what we had heard internally and externally to sort of say, "Yeah, that reflects what we believe are the places people value on the forest." But I don't recall any new conversations like "Oh, this one is different." It didn't show up succinctly. But we did look at it and kind of compare it to what we felt we knew already. 
That the VCA analysis was of little use may be due to several factors. The VCA methodology basically quantifies and maps public values in a system adopted from ecology. The utility of the data may have been unrecognized in the context of "social" science, as it does not conform to the typical ongoing participatory workshop-based methodologies used to collect and assess public preferences during the planning process. In addition, the researchers may have (or may not have) communicated to the Coconino planning team that the data is more that just locating special places on a map (which simply confirmed what was already known). There does not seem to have been an associated "how to" manual that provided team members with specific instructions on how to use and interpret the data in combination with other modeling strategies using GIS-based technology, for example how to add VCA layers to vegetation models or the recreation opportunity spectrum to inform draft plan alternatives.

Timing may also have played a part in dismissing this data. As many forest-level staff involved in plan revision have mentioned, the pressure to complete the process in a timely manner with finite resources (especially for forests using the 1982 planning rule who were under pressure to finish), is not conducive to developing expertise in a new methodology, much less trying to figure out how to integrate the data into a planning process well underway. If innovative tools, data and methodologies are to be adopted in times of heightened organizational pressure, they must reduce the work load or provide a proven track record of bettering an outcome, not add to the burden. 


\section{Appendix G: Francis Marion National Forest Brief}

\section{FRANCIS MARION NATIONAL FOREST (South Carolina)}

\section{FOREST DESCRIPTION}

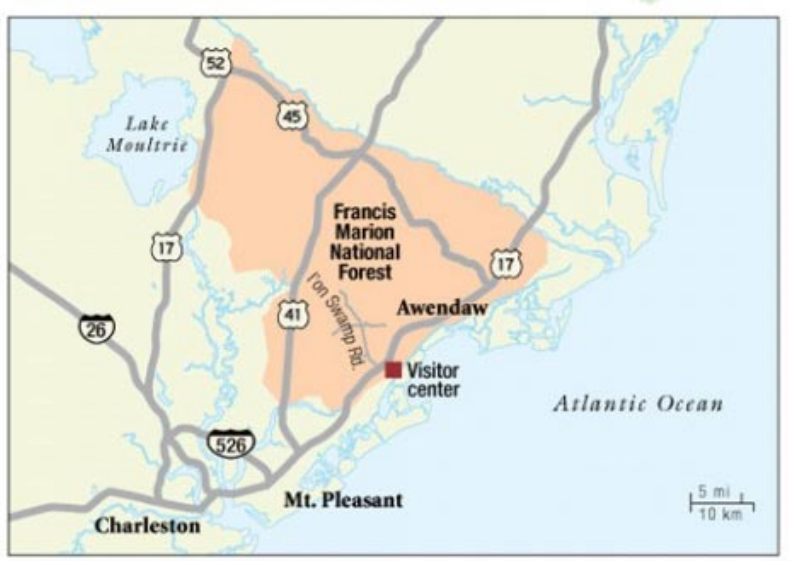

Officially designated in 1936, the Francis Marion National Forest abuts the Atlantic Ocean coastline of South Carolina. Though relatively small (approximately 260,000 acres), the forest contains unique and fragile environmental features, such as coastal cypress forests and limestone sinks, and is home to several endangered species. Four designated wilderness areas protect sensitive habitats, including 25 pristine Carolina bays.

The Forest has a rich cultural history as

well. There is evidence that prehistoric Native Americans occupied the area over 4,000 years ago. The Forest's namesake, General Francis Marion, fought the British in its swamps during the Revolutionary War. Artifacts from the Civil War still dot its hills, valleys and coastlines. Today, the area surrounding the Forest is primarily urban and growing fast. Charleston, with a population of close to 130,000 in 2013, is the largest city in the region.

Early in the $20^{\text {th }}$ century, management activities focused on growing high-quality pine sawtimber as the chief crop. The first official forest management plan, released in 1985, retained this historic focus on timber production. However, Hurricane Hugo (in 1989) leveled more than a third of the forested areas, damaged sensitive ecosystems, and created new management challenges. For example, about $60 \%$ of the pine stock received heavy or moderate damage and a sizeable percentage of the bottomland hardwoods were broken or uprooted. The subsequent growth of a dense understory after the hurricane created a heightened threat of catastrophic fire. The population of the endangered redcockaded woodpecker, which relies on standing trees for nesting, plummeted. Understandably, 1996 amendments to the forest plan focused on recovery efforts from the hurricane.

Forest management priorities today have shifted to recreation and environmental protection. Timber harvesting has largely been replaced by thinning of understory vegetation and other activities that produce biomass for local energy production. Recovery efforts after Hurricane Hugo raised public awareness of fragile ecosystems and spawned several local interest groups invested in forest restoration and protection. Rapid growth in the surrounding urban population (in both diversity and size) has put increasing 
pressure on existing infrastructure and recreational facilities. The NVUM survey in 2008 , for example, indicated that, on average, 430,000 people visit the forest on an annual basis. Public outreach in the fall of 2012, in preparation for developing a new forest management plan, identified several themes of interest including (1) better integration of stakeholders and interest groups in the planning process; (2) protection of features (natural and cultural) unique to the forest; (3) enhancing the ability of the forest to contribute to quality of life by providing a wide range of recreational opportunities and improved human-nature connections; and (4) addressing challenges posed by the wildland-urban interface, primarily fire management and water quality.

Francis Marion began its most recent forest plan revision in October, 2012, under the new 2012 forest planning rule (though not an official early adopter), completing its assessment in December, 2013. The formal Notice of Intent was filed in April, 2014, with the Draft Plan and DEIS following in September, 2015. After an intense four-year process, the Final Plan, FEIS, and draft ROD were submitted in January 2017. Since submission, the Francis Marion Final Plan has logged four amendments, two of which added additional Species of Conservation Concern (SCC) to the management plan.

\section{Francis Marion Plan Revision Timeline}

1. Assessment Report (December 2013)

2. Public Scoping/Sensing (Oct-Dec 2012; Feb 2013; Feb-March 2014)

3. Need to Change (January 2014)

4. Notice of Intent (April 2014)

5. Draft Plan \& DEIS (September 2015)

6. Final Plan \& FEIS (January 2017)

7. Amendments to Final Plan (June 2018) - adds Species of Conservation Concern

\section{FOREST PLAN REVISION \& PUBLIC ENGAGEMENT}

Geographic scope of public participation: Dozens of meetings took place during the plan revision process as part of an intense scoping and sensing effort before and during the draft planning. Charleston and Mt. Pleasant, the largest metropolitan areas in the region, hosted at least two meetings. Special topic forums, focusing on ecological issues, recreation, and at-risk species protection, were held at the Forest Supervisor's office. Other public meetings occurred in small towns around the region. Comments made by interviewees, however, indicated that Forest staff (many of whom can boast a very long tenure) have developed strong and positive working relationships with surrounding communities, elected officials, and the numerous special interest and advocacy groups. Interviewees were confident that outreach efforts provided sufficient opportunities for face-to-face interaction between the Forest planning staff and local-regional stakeholders with numerous locations, forums and mediums for the public to provide meaningful and spatially explicit input. 


\section{SOCIO-CULTURAL AND SOCIO-SPATIAL DATA IN PLAN REVISION}

Social Science, Socio-Spatial Data and the Assessment Report: The sociological, cultural and economic chapters in the Assessment Report (compiled by two economists and a social scientist contracted through TEAMS) contain the typical demographic and economic analyses derived from U.S. Census, Department of Labor, Department of Commerce, and State socio-economic databases - a coarse analysis concentrating on seven metropolitan communities surrounding the Forest. In the Assessment Report, nonmaterial benefits and public values were encapsulated by the statement, "it is not feasible to estimate non-market values during the planning process; it is important for Forest management to recognize that the true value of Forest resources include both market and non-market values so that they can make more informed decisions regarding their use" (p. 302). The Assessment Report does not display or cite any maps or data tables showing the socio-spatial data collected either from the public meetings or the online crowdmapping tool (described below). However, the report narrative does contain several references to these activities and occasionally short summaries of the dataparticularly in the social sustainability and cultural services sections. Of note, data collected from the public meetings and online tool, though not reported in great detail, were specifically noted as relevant sources of "best available science" (p. 9).

Socio-Spatial Data and Public Participation: The Francis Marion used an outside contractor to design and implement a public engagement strategy. Public meetings and workshops occurred during compilation of the Forest Assessment Report through the release of the Draft Plan and DEIS (October, 2012 to December, 2015). Public engagement included mapping activities as well as online mapping tools for broader public outreach (based on the Crowdbrite platform and, later, the Talking Points Collaborative Mapping tool - or TPCM). Public meetings, in 2012, used the world café model with an information session and small

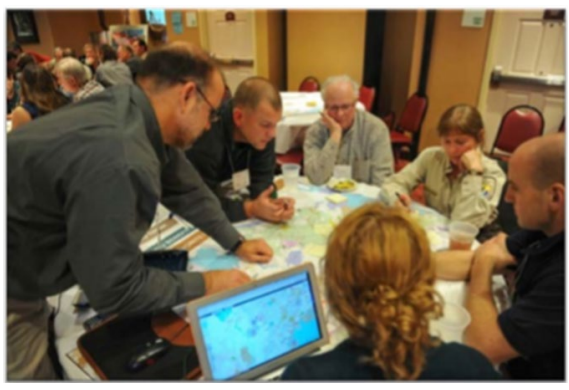

Mapping My Favorite Places group discussions focusing on specific questions and issues. In 2013 and 2014, this model transformed into what was termed "community conversations," which consisted of a series of canvases with prompting questions that gathered both spatial and non-spatial data about (1) the benefits the public receives from the forest; (2) what is unique and special about the forest; (3) the public's favorite places to recreate and commune with nature; and (4) what might make a visit to the forest better. 
Socio-Spatial Data and the Draft Plan: A detailed description of the mapping activities and public comments collected during the Francis Marion plan revision process can be found in the Public Workshop and Online Engagement Summary Report through a link on the Forest's website. An analysis of how the socio-spatial data collected informed the planning process is more thoroughly addressed in Geographic Areas \& the Land Management Planning Process, an unpublished report prepared by the Recreation Sites Program Lead in the Region 8 Atlanta office. This report uses the Francis Marion plan revision process as a case study in which to explore how "geographic areas" (in the language of the planning rule) or "Resource Integration Zones" (as they came to be called in the Draft Plan) could serve as a tool to integrate services/benefits and develop place-based management strategies in keeping with the intent of the 2012 rule. The report indicates that the public workshops, particularly those that took place in 2013 and 2014, and the online collaborative mapping tool proved to be a source of critical information for

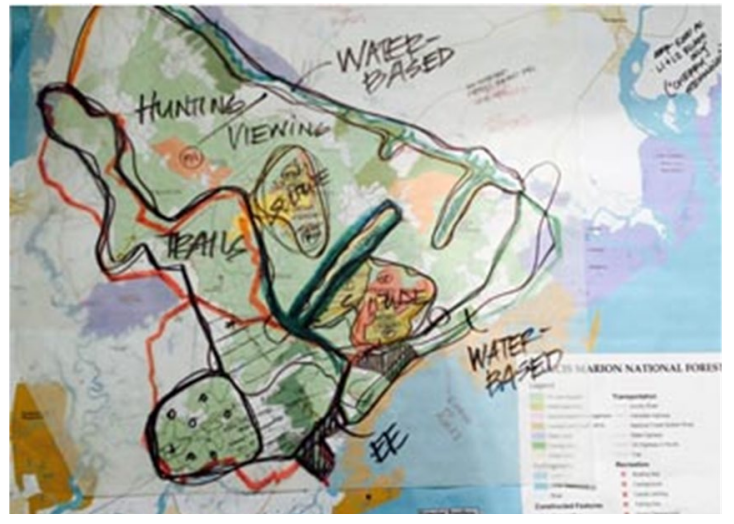

Mapping Recreation Areas understanding the spatial dimensions of recreational use of Forest landscapes by the public - what uses were important to them and where these uses took place. The data were integrated into the Draft Forest Plan through identification of several Resource Integration Zones with targeted management priorities and strategies. These zones were determined by combining the spatial extents delineated in the recreation and special places public mapping activities, as shown in the graphic, with other spatial layers such as the Recreation Opportunity Spectrum, the Scenic Integrity Analysis, and various infrastructure and biophysical GIS layers.

\section{HIGHLIGHTS OF THE FRANCIS MARION PLAN REVISION PROCESS}

Sensing, Scoping and Community Conversations: The Francis Marion committed early to providing a comprehensive, multi-tiered public engagement process throughout plan development in a process they termed "community conversations." In large part, this process was facilitated by an outside contractor. Comments from interviewees indicate that this investment was critical in both gathering data on public preferences and values to inform plan components as well as creating a shared vision of management of forest resources. Of note, the forest released preliminary copies of both the draft and final plans to solicit public input. This strategy is not always adopted by forests as it can result in a time-consuming feedback loop in which plan components are constantly being reassessed and revised based on public comments. However, planning team members felt that the close working relationships forest staff had developed with local interest groups and forest users over decades and the heavy focus on public recreational use of the forest necessitated reaching out and fully engaging stakeholders and constituencies throughout the plan development process. As is noted above, these conversations and particularly 
the public mapping workshops resulting in rethinking management areas as "resource integration zones." As one team member reflected:

We had sensing calls - we had people calling and talking at length about what was important to them in this planning cycle... We did some online crowdsourcing. We used Crowdbrite, which was an online mapping, sort of a community engagement, tool... Gosh, you know, the best thing is just the one-onone information, like calls and working with partners. From a community standpoint we had really good information...we really got going on this sense of community and these community nodes and these, we called them, crossroad communities.

\section{DISCUSSION}

We are "telling a story": The Francis Marion used contractors from the Enterprise TEAM system to conduct much of the social and economic analysis. This study interviewed two of these scientists. Both approached this task with a vision that economic analysis and vulnerability assessments and other socio-cultural data-gathering are more than just numbers. To have value, they must be accompanied by the "story" about what all those numbers mean. This represents an opportunity to acknowledge and elevate community needs and values in forest management especially as forests enter the monitoring stage. As one scientist mused:

I think what is really of value for our publics isn't the numbers, but rather the narrative and the interpretation of those numbers. It's talking about contributions to local communities in terms of jobs, which is interesting...People care about the fact that those jobs have been there for years and they're a part of their local identity. That's the qualitative interpretation of the importance of those

jobs. That is what matters, not their small absolute value...I think it's at the crux of the new rule...I don't think that it's really saying that we do anything new that we haven't done before, but that we need to tell a story in a way that we haven't been telling before. It's emphasizing the fact that we need to be talking about our beneficiaries so that we are managing for forest health. But, also, how does managing for forest health benefit communities? How does doing the things we do really benefit communities in a way that we haven't been acknowledging or telling? 


\section{Appendix H: Second Level Codebook}

\section{FACTORS IN THE USE OF SOCIO-SPATIAL DATA IN NATIONAL FOREST PLANNING 2ND LEVEL THEMATIC CODEBOOK}

EXTERNAL: References to federal laws, regulations and rules that represent the legal framework in which USFS operations occur, such as NEPA, ESA, OMB, MUSY or NFMA. This also refers to references about external political forces, either overt or covert, such as "litigation or conflict mentality" (referring to the choice of external organizations to litigate rather than collaborate). DO NOT use this code for references to internal USFS standard operating protocols and procedures such as what can be found in the Directives.

Policy: Public priorities regarding forest management (or other natural resource management) that provide the context in which forest management occurs (e.g. protection of endangered species or stakeholder preferences for recreation or timber production). References to governance issues, political will or Congressional demands.

Litigation: References to appeals and lawsuits regarding forest planning decisions. OMB: References to the regulations for collecting survey data from the public (use this code even if the reference does not specifically mention the $\mathrm{OMB}$, but is implicit in the statement).

NEPA: References to regulations and protocols for creating environmental impact statements or other stipulations in NEPA, such as public comment period.

FACA: References to regulations contained in the Federal Advisory Committee Act, particularly in relationship to associations with collaboratives. Use this code only if FACA is specifically mentioned - not for general references to working with collaboratives (see Public Participation).

ORG STRUCTURE: References to how roles, responsibilities and authorities are defined, assigned, controlled and coordinated within the agency to achieve organizational goals.

Integration: References to relationships between the different agency units within the USFS - Forests, Regional Offices, Research Stations, and Other (Washington Assigned, Enterprise Teams).

Infrastructure: References to the physical systems within the agency, such as computer capabilities; IT support.

Org Strategy: References to the formal rules around the definition of tasks, coordination of functions among employees and units (e.g. centralization or decentralization; insulation or silos; oversight functions) and standard operating procedures.

Capacity: References to agency staffing levels, extent and diversity of expertise, staff turnover, other stressors on staff time (e.g. fire management, etc.).

Budget: References to sources of funding (for either planning or research) including references to budget cycles, amounts for operational function areas, and other allocations. Leadership: References to the hierarchical structure within the unit, role of agency leaders, including DC leadership, station or program directors, line officers (e.g. oversight briefings), references to "champions" within leadership for human dimensions or social science. Do not use this code for references to organizational decision authorities.

Org Decisions: References to how decisions are made within the organization or unit, including decision-making authorities. Include both formal and informal decision-making practices and protocols. 
Communication: References to protocols and processes for dissemination of information within agency units, between units, and externally to stakeholders or other parties.

Incentives: References to employee incentive structures, both formal and informal, in relation to performance evaluations, promotions. This code includes references to organizational "efficiency" if stated within the context of staff expectations (e.g. the agency expects planning to occur in an "efficient" and "timely" manner).

Resilience: References to the ability of the organization to adapt to changing circumstances.

ORG CULTURE: References to the shared assumptions, values, and beliefs which govern how employees behave in the agency, particularly how they are expected to interact and perform their jobs.

Behaviors: References to behavioral and other "personal" norms and ideas that influence how agency employees approach their work (e.g. risk aversion).

Habits: References to formal and informal "belief systems" embedded within the agency that provide a framework for how employees conduct themselves and perform their tasks (e.g. habits of planning; habits of research; culture of science). Do not include references to standard operating protocols (these are the "written" instructions).

PLANNING: References to formal forest planning processes, protocols, rules, regulations; references to the perceived challenges and opportunities in forest planning; interviewee involvement in the planning process (e.g. team leader).

Directives: Specific references to the Forest Service Directives (for either the 1982 or 2012 Planning Rule).

Planning Rule: Specific references to the Planning Rule (1982, 2012 or others) including comments about similarities or differences between the rules and comments about rule components (e.g. ecosystem services, cultural services, sustainability, public engagement, adaptive management).

Planning Decisions: References to the planning process itself; how planning decisions are made in the context of the planning rule (e.g. logistics, setting up meetings); creation of the planning documents (e.g. assessments). NOTE: This is a generic category to link PLANNING with other areas of discussion.

Timing: References about timing and scheduling of forest planning stages and the challenges or opportunities encountered (e.g. the "dark hole").

PUBLIC ENGAGEMENT: References that relate to public outreach, public participation in the planning process (including involvement of collaboratives) and participatory processes put in place during planning.

Outreach: References to the methods and models used in public outreach during the planning process.

Transparency: References to the extent to which information about the planning process is shared with the public and how information gathered from the public influences planning decisions.

Trust: References to opinions and observations about public reactions to and about the planning process, outreach methods, data collected. Includes references to public needs regarding ongoing engagement to accommodate new people, new needs, new issues (e.g. collaborative turnover, institutional memory); building ongoing relationships with stakeholders and other publics. 
Collaboratives: References to interaction with and methods used to engage formal organized stakeholder and interest groups during the planning process (as opposed to general outreach to different "publics").

DATA COLLECTION: References to the collection and/or use of data (particularly social, economic and cultural data) in the assessments or other planning documents. Includes the use of data collection tools (e.g. PPGIS, IMPLAN, NVUM, etc.).

Spatial Data: References to the types of socio-spatial data collected for assessments, draft/final plans, or public outreach and communication; identified gaps in socio-spatial data; discussions about the scale of socio-spatial data.

Data Type: References to qualitative versus quantitative data, social versus biophysical data. Also includes discussions about data gaps. DO NOT use this code for discussions about spatial data such as sense of place or special places (use the $<$ Spatial Data $>$ code).

Data Tools \& Methods: References about different methods and tools for collecting data and the effectiveness or challenges in using those methods. DO NOT use this code for specific references to mapping tools (use <Mapping> code).

Mapping: Specific references to types of mapping tools used to collect socio-spatial data.

SCIENCE: References to science in general including interviewee's familiarity with scientific principles and methods, the role of science in the interviewee's job, collaboration and networking among and between scientists; descriptions or opinions about where science (social and/or natural) fits into the planning process.

Research: References about important research to focus on in the future; up and coming topics areas in forest research.

BASI: References to how the Best Available Scientific Information is determined in the forest planning process (e.g. formal definitions); any opinions or ideas about the criteria for determining how socio-cultural, place-based, or publicly acquired data meets the criteria for BASI.

Scientific Paradigms: References to scientific paradigms as they relate to or are used in planning processes (e.g. recreation carrying capacity, vulnerability analysis, ecological/ecosystem integrity).

Networking: References to how scientists interact with each other (social and natural scientists) including challenges or opportunities related to communication between scientific disciplines or doing integrated research. 


\section{Appendix I: Final Codebook}

\section{FACTORS IN THE USE OF SOCIO-SPATIAL DATA IN NATIONAL FOREST PLANNING FINAL CODEBOOK}

\begin{tabular}{|c|c|c|}
\hline LABEL & DESCRIPTION & EXAMPLE TEXT \\
\hline \multicolumn{3}{|l|}{ EXTERNAL } \\
\hline Policy \& Law & $\begin{array}{l}\text { References to federal laws, regulations } \\
\text { and rules that represent the legal } \\
\text { framework in which USFS operations } \\
\text { occur, such as NEPA, ESA, OMB, PRA, } \\
\text { MUSY, NFMA or FOIA or other broad } \\
\text { comments about law and policy. } \\
\text { References about the } 1982 \text { or } 2012 \text { Forest } \\
\text { Planning Rule. References about external } \\
\text { political forces, either overt or covert, } \\
\text { such as comments about "policy-makers," } \\
\text { "Congress, "legislators." } \\
\text { This code should NOT be used for } \\
\text { references to internal USFS standard } \\
\text { operating procedures, such as what can } \\
\text { be found in the Directives. }\end{array}$ & $\begin{array}{l}\text { Part of it is figuring stuff out for the first time. } \\
\text { What I mean by that is things like a PPGIS protocol. } \\
\text { Clearly it brings a lot of value to the forest plan } \\
\text { revision process. But, corporately there's not a lot } \\
\text { of organization about how to do that, how to steer } \\
\text { clear of OMB requirements. You know we need } \\
\text { this OMB clearance. Oh my god, if I could ask for } \\
\text { one thing it would be that! }\end{array}$ \\
\hline \multicolumn{3}{|c|}{ ORGANIZATIONAL STRUCTURE } \\
\hline $\begin{array}{l}\text { Org Strategy } \\
\text { Roles \& } \\
\text { Responsibilities } \\
\text { Communication } \\
\text { SOPs } \\
\text { Formal } \\
\text { Incentives }\end{array}$ & $\begin{array}{l}\text { References to the procedures and rules } \\
\text { around definition of tasks/functions, } \\
\text { coordination/communication between } \\
\text { employees and units (e.g. } \\
\text { centralization/decentralization; insulation } \\
\text { or silos; oversight functions), standard } \\
\text { operating procedures, employee incentive } \\
\text { structures (both formal and informal) in } \\
\text { relation to performance evaluations or } \\
\text { promotions and expectations on how } \\
\text { USFS levels interact. } \\
\text { This code includes references to } \\
\text { organizational "efficiency" if stated within } \\
\text { the context of formal incentives. Do NOT } \\
\text { use this code if "efficiency" is referenced } \\
\text { as an expected behavior (use ORG } \\
\text { CULTURE code). }\end{array}$ & $\begin{array}{l}\text { On staff incentives: The other thing is that there } \\
\text { are differences between what is science and what } \\
\text { is not science in terms of writing a report about } \\
\text { something. The National Forest System needs } \\
\text { those reports. They need to see the documents. } \\
\text { But, for a scientist to work on some of these things } \\
\text { would be a career killer. If they can't get research } \\
\text { papers out they will not be successful the next time } \\
\text { they go to panel. }\end{array}$ \\
\hline $\begin{array}{l}\text { Capacity } \\
\text { Staffing } \\
\text { Levels/Turnover } \\
\text { Available } \\
\text { Expertise } \\
\text { Ability to Adapt }\end{array}$ & $\begin{array}{l}\text { References to agency staffing levels, } \\
\text { extent and diversity of expertise, staff } \\
\text { turnover, other stressors on staff time } \\
\text { (e.g. fire management vs. forest } \\
\text { management, etc.). References to the } \\
\text { capacity (or willingness) for staff to adapt } \\
\text { to changing circumstances. }\end{array}$ & $\begin{array}{l}\text { So how much can you anticipate? How much do } \\
\text { the line officers need to be involved versus say the } \\
\text { forest planner who's leading the effort? How much } \\
\text { can the team take on? A lot of it is needing to be } \\
\text { adaptive and understanding what resources are } \\
\text { available to then also be responsive. We were able } \\
\text { to accommodate some things, like the reporter }\end{array}$ \\
\hline
\end{tabular}




\begin{tabular}{|c|c|c|}
\hline Resilience & & $\begin{array}{l}\text { who wanted to visit all of the areas we were } \\
\text { considering. I think we gave him a map, but said, } \\
\text { "We don't have the resources to go out with you on } \\
\text { the forest." }\end{array}$ \\
\hline Budget & $\begin{array}{l}\text { References to sources of funding (for } \\
\text { either planning or research) including } \\
\text { references to budget cycles, amounts for } \\
\text { operations and functional areas, money as } \\
\text { incentive (e.g. research grants), and other } \\
\text { financial references. }\end{array}$ & $\begin{array}{l}\text { Budgets have become more and ... how do I want } \\
\text { to say this ... more and more of our budget goes to } \\
\text { fire suppression and that comes at a cost of } \\
\text { everything else, right? So currently, over } 50 \% \text { of the } \\
\text { FS budget goes to fire suppression. There's a } \\
\text { transaction cost involved there. So, updating } \\
\text { maps? Kind of falls to the bottom of the pile, right? }\end{array}$ \\
\hline $\begin{array}{l}\text { Leadership } \\
\text { Authority } \\
\text { Hierarchy } \\
\text { Decision-Making }\end{array}$ & $\begin{array}{l}\text { References to the hierarchical structure } \\
\text { within the unit, role of agency leaders, } \\
\text { including DC leadership, station or } \\
\text { program directors, line officers (e.g. } \\
\text { oversight, HD "champions"), decision- } \\
\text { making/makers. }\end{array}$ & $\begin{array}{l}\text { So, one, it helps that the regional office provides } \\
\text { some of those resources. The forest leadership } \\
\text { team has said that forest planning revision and the } \\
\text { amendment work we're doing for the neighboring } \\
\text { forest - that's part of what we're doing - is the \#1 } \\
\text { priority. If we're going to do this, we're going to } \\
\text { get in and get out and do this as quickly and in the } \\
\text { best way that we can. So that has helped to } \\
\text { prioritize revisions \#1. }\end{array}$ \\
\hline \multicolumn{3}{|c|}{ ORGANIZATIONAL CULTURE } \\
\hline Org Culture & $\begin{array}{l}\text { References to the shared assumptions, } \\
\text { values, and beliefs which govern how } \\
\text { employees behave in the agency, } \\
\text { particularly how they are expected to } \\
\text { interact and perform their jobs. Includes } \\
\text { accepted habits (e.g. habits of planning; } \\
\text { culture of science; professionalism) or } \\
\text { informal ways of behaving (e.g. risk } \\
\text { aversion). } \\
\text { These are comments indicating "this is } \\
\text { how we are (or feel we are) expected to } \\
\text { do our job" as long as it does NOT } \\
\text { specifically relate to formalized operating } \\
\text { procedures (use ORG STRUCTURE codes). }\end{array}$ & $\begin{array}{l}\text { I also get to the point where I think we've got to } \\
\text { put some common sense rules in place. And to me } \\
\text { that comes to the point where data becomes less } \\
\text { important than just putting some basic rules into } \\
\text { place about how you go about planning and then } \\
\text { adapting as you go. That's a hard thing for this } \\
\text { agency to deal with - that thought - because we're } \\
\text { very much scientists and we want to have } \\
\text { everything perfected on that front. It's hard to get } \\
\text { all of these resource specialists to let go of that... } \\
\text { The public gets it. It's internally - the agency } \\
\text { specialists - that struggle with it. I say, we're } \\
\text { gonna get stuff wrong. We are gonna get shit } \\
\text { wrong! It's how they frame it in their heads...But } \\
\text { we get paralyzed by this stuff and it's all internal. }\end{array}$ \\
\hline \multicolumn{3}{|c|}{ PUBLIC ENGAGEMENT } \\
\hline $\begin{array}{l}\text { Public } \\
\text { Engagement }\end{array}$ & $\begin{array}{l}\text { References to public outreach and } \\
\text { collaboration (both informal \& formal } \\
\text { interest groups) and opinions about } \\
\text { methods used to conduct public outreach } \\
\text { and disseminate information to the public } \\
\text { during the planning process (e.g. public } \\
\text { meetings, comment periods, etc.); include } \\
\text { comments about how public outreach } \\
\text { affects the planning process as well as } \\
\text { observations about public reactions to and } \\
\text { about the planning process, including } \\
\text { references to public needs, } \\
\text { accommodations, challenges, }\end{array}$ & $\begin{array}{l}\text {...I think we worked really hard to broaden the } \\
\text { involvement and we even partnered with the } \\
\text { city...So we partnered and engaged in a } \\
\text { conversation about sustainability for the } \\
\text { community and the associated forest. Out of that } \\
\text { we had some workshops, sort of a big capstone } \\
\text { event on the weekend where we tried to invite a } \\
\text { broad range of stakeholders to come and talk } \\
\text { about sustainability... We put some of the results in } \\
\text { our reports, but there was also a new friends group } \\
\text { - "Friends of the Forest" kind of group - that } \\
\text { formed out of it... We were trying really hard to } \\
\text { engage the public, for those who were interested. }\end{array}$ \\
\hline
\end{tabular}




\begin{tabular}{|c|c|c|}
\hline & $\begin{array}{l}\text { opportunities and trust between agency } \\
\text { and stakeholders. } \\
\text { Do NOT include references about actual } \\
\text { data collection methods and tools (Use } \\
\text { DATA COLLECTION codes). }\end{array}$ & \\
\hline \multicolumn{3}{|c|}{ DATA COLLECTION } \\
\hline Spatial Data & $\begin{array}{l}\text { References to the types of socio-spatial } \\
\text { data collected for assessments, draft/final } \\
\text { plans, or public outreach and } \\
\text { communication; identified gaps in socio- } \\
\text { spatial data; discussions about the } \\
\text { geographic scale of socio-spatial data. } \\
\text { Do NOT use this code for comments about } \\
\text { socio-cultural data in general, only for } \\
\text { specific references to "socio-spatial" data } \\
\text { and its use as defined in this study (public } \\
\text { participatory). Use DATA GENERAL code. }\end{array}$ & $\begin{array}{l}\text { Well, taking the recreation one where we asked } \\
\text { people to identify their special places, I think that } \\
\text { was a hit and a miss with that, maybe in just the } \\
\text { way we did it. We had maps on the wall and we } \\
\text { said, "Just draw a circle around what your favorite } \\
\text { place is and tell us what recreational activity you } \\
\text { do in that place." And people either drew great big } \\
\text { huge circles or they did a dot. So the usefulness, } \\
\text { sort of like the rigor of that information, may not } \\
\text { have been really great. }\end{array}$ \\
\hline Data General & $\begin{array}{l}\text { General references to the collection and } \\
\text { use of data including qualitative vs. } \\
\text { quantitative data, social vs. biophysical } \\
\text { data. Also includes comments about data } \\
\text { gaps. Include references about different } \\
\text { methods and tools for collecting data and } \\
\text { the effectiveness or challenges in using } \\
\text { those methods. Include specific } \\
\text { references to types of tools used and what } \\
\text { kind of data is collected like the NVUM, } \\
\text { ROS, scenic valuation tools, etc. that do } \\
\text { not explicitly use public participatory data } \\
\text { collection methods. } \\
\text { Do NOT include specific references to } \\
\text { socio-spatial data or use of mapping tools } \\
\text { (use SPATIAL DATA code) }\end{array}$ & $\begin{array}{l}\text { I find it to be really a person by person thing. If } \\
\text { you've got somebody out there on a forest or a } \\
\text { district who is interested in or not - not "allergic" } \\
\text { to social science - it works great because they kind } \\
\text { of get it and they're going to, you know, take it on } \\
\text { and embrace it and go with that. But, if you get } \\
\text { people who just think it's going to be hard and } \\
\text { they make it hard, I don't know if they want to, but } \\
\text { they see it as they can get away with the bare } \\
\text { minimum that's acceptable. We are an agency of } \\
\text { natural resource people, that's the folks who are } \\
\text { out there doing their job. So a lot of times you run } \\
\text { into this social science thing...it's like, "Arrrgghhh!" } \\
\text { No, they'd rather be outside in the field doing their } \\
\text { thing not talking to people or about people. }\end{array}$ \\
\hline \multicolumn{3}{|l|}{ SCIENCE } \\
\hline $\begin{array}{l}\text { Research \& } \\
\text { BASI }\end{array}$ & $\begin{array}{l}\text { References to how the Best Available } \\
\text { Scientific Information (BASI) is determined } \\
\text { in the forest planning process (e.g. formal } \\
\text { definitions); any opinions or ideas about } \\
\text { the criteria for determining how socio- } \\
\text { cultural, place-based, or publicly acquired } \\
\text { data meets the criteria for BASI. } \\
\text { References about important research to } \\
\text { focus on in the future; up and coming } \\
\text { topics areas in forest research. }\end{array}$ & $\begin{array}{l}\text { So, you know the best available science is basically } \\
\text { determined through convention, in that, again, you } \\
\text { know we're not doing anything new... Under the } \\
\text { new rule, the } 2012 \text { rule, we have some different } \\
\text { contexts that we're looking at...I think the science is } \\
\text { really convention in that we have been } \\
\text { characterizing benefits to people from forest lands } \\
\text { and public lands for quite some time. It's also } \\
\text { narrative. There's some secondary sources out } \\
\text { there in terms of best available science - in terms } \\
\text { of characterizing those benefits and services - that } \\
\text { are really from public input and public } \\
\text { engagement. There's no one single analysis } \\
\text { process. }\end{array}$ \\
\hline
\end{tabular}




\begin{tabular}{|l|l|l|}
\hline Networking & $\begin{array}{l}\text { References to how scientists interact and } \\
\text { network with each other (social and } \\
\text { natural scientists) and how scientists } \\
\text { interact with planners and other } \\
\text { practitioners. Include references to } \\
\text { challenges or opportunities related to } \\
\text { communication between scientific } \\
\text { disciplines when doing integrated, inter- } \\
\text { disciplinary research. }\end{array}$ & $\begin{array}{l}\text { I think the only tension is first that every expertise, } \\
\text { every group has their own language. And that's } \\
\text { one thing that when I first started working with } \\
\text { some social scientists, I felt like I was teaching } \\
\text { them a whole new language and they're teaching } \\
\text { me a whole new language. So, in some ways it's } \\
\text { just a matter of how you've been trained in a large } \\
\text { system, how you perceive the world and to express } \\
\text { your understanding of it...And I know that the } \\
\text { National Forest System really values social science } \\
\text { and really wants to be informed, not necessarily } \\
\text { telling them biologically how many trees you can } \\
\text { cut down, but what are public perceptions about } \\
\text { cutting down trees. What is it that people really } \\
\text { want? Do they really want jobs or do they really } \\
\text { want untouched landscapes? What is it that they } \\
\end{array}$ \\
& $\begin{array}{l}\text { value? And that helps guide public policy. } \\
\end{array}$
\end{tabular}


Appendix J: Analytic Meta-Memo

Factors in the Use of Socio-Spatial Data in National Forest Planning

ANALYTIC META-MEMO: NATIONAL FORESTS

1. What seems most important or significant to these participants? What stood out most in the interviews?

Lack of CAPACITY (expertise, timing, budget) is a constant theme (...wish we could have done more; wish I could have done it earlier in the process; there was never enough time; low hanging fruit).

PUBLIC INPUT (including values and special places) IS accepted as a critical part of the forest plan revision process and planners and ID Team members made significant efforts to gather this kind of information.

You know, I think we are making big strides toward this more collaborative process of us having people involved and helping kind of guide where we're going, what we're doing, and that sort of thing. I think that that's going to be the only way we're going to be able to survive. It's important to have that public support for public lands. And I really feel like that's the only way that we're going to get it is by having people involved.

\section{SOCIO-SPATIAL DATA:}

- Public needs to "tell us" what benefits they get from the forest, what makes it special, what they want to see done within the forest.

- The socio-spatial data gathered DID influence plan components in myriad ways (though it wasn't part of traditional modeling via ROS, SMS, that were highlighted in the assessments).

- Mapping activities at public workshops and information sessions were important engagement tools and not necessarily seen as data collection tools.

- The engagement doesn't/shouldn't end once the management plan is finished (e.g. monitoring strategy + teaching/learning from the public).

2. How did the participants talk about the use of social science and/or socio-spatial data or data gaps? Was it said in a positive, negative or neutral context?

Used the Tools at hand for Socio-Spatial Data Collection:

So, we used the collaborative mapping tool primarily for the wilderness evaluation process. So what are the values? What type of wilderness character do you see that's relevant in these areas so we can incorporate that into our evaluation? I think it was a new tool and probably confused people a little. But, I just love being able to provide the levels of information that we think are important so the public could layer those on the map see all of that. That was a really cool tool. 
Qualitative Data and the Story:

Well, I'm kind of unique, I think, in terms of an economist within the agency. But, I really put a high value on the qualitative. I think a lot of the numbers that economists use within the agency are just, yeah, they're no different than what most economists use. But I think what is really of value for our publics isn't the numbers, but rather the narrative and the interpretation of those numbers. It's talking about contributions to local communities in terms of jobs, which is interesting. But in most cases, the agency contributes a pretty small portion of jobs to particular forest related industries. That's not what people care about. People care about the fact that those jobs have been there for years and they're a part of their local identity. That's the qualitative interpretation of the importance of those jobs. That is what matters, not their small absolute value.

\section{Was there anything in the interviews that surprised you? What insights did you} gain?

Is it really the responsibility of the USFS to collect all this public data?

I would say that there's high interest in that kind of information [socio-spatial data] from county commissioners, from some people in the community, business people. They're wrestling with it every day...I don't know if the social, more of a recreation trends and some of those other social things, some of those other drivers of why things are not great economically, it can't be, in my own opinion, it can't be all on the shoulders of the federal lands. I mean, that's a piece of the puzzle, but there's so many other drivers as to why things are shifting away from certain things, and increasing value of like water and those other ecological services.

\section{Did the participants talk about significant links, challenges or opportunities? What were they?}

OPPORTUNITY: Collecting Public Data = Better Engagement \& Management Decisions

And then the last one was drawing lines on the map [the last step in a public engagement/mapping outreach. What should be the management areas we should be looking at? What would you like to see out here? And you know, ultimately, the goal of this was the kum-bah-yah moment when they all came together and said, 'yes! If you want this and I want this then we can get a win-win!' And I talked to them for 10 months straight 'tell us what you want from your government.' I'm telling you, you would never think it possible, but this is exactly what you want your government doing, asking the public what they want instead of taking information then we go in our black box and we do something with it...And I kept saying, 'you guys have to get this because this is your opportunity. It's going to be this time frame to here [hand gesture from one end of a spectrum to another]. It's your opportunity to contribute. If you don't choose to do that then you're giving the responsibility back to us and we will have to sort 
out what that greatest good is. That's what we're going to try to find here - the greatest good.

So I think, to me, these people are engaged early with what these communities want, not necessarily want from the Forest Service, but how do they gauge their community health? What do they think are healthy communities? We shouldn't be there to tell them what a healthy community is. They should be telling us. And that way there is some shared vision over that. So it's interesting to be more engaged and the Forest Service doesn't always do that real well. You know, we're pretty insulated. So sometimes it feels like we just do our own little thing until we do a forest plan and then all of a sudden we're like "hey, we want everybody's opinion." So I think it would be better if we had done some of that community sensing and those community connections earlier.

...there were some ideas that emerged in that process that were dramatically different than what we were thinking originally and we used that information. We changed management areas. We completely retooled our canned set of management area allocations to better reflect front country recreation - front country recreation is where this developed recreation is going to be more predominant because that's what they were saying to us. 'We want day use opportunities that are open to us. We want our mountain bike opportunities in the front country, and we want you to recognize that, instead of putting me in the backcountry. We don't know if you're going to allow our opportunities to continue to be there in 50 years.' So we really retooled based on that feedback.

LINK/CHALLENGE: Socio-Spatial Data - Capacity/Time/Budget I don't think the rules in place had really anything to do with it. I think it was an ability with personnel and timing that the forest itself does not have that skill set...I don't think there was anyone that said don't waste your time, nobody ever told us that. And actually some of the county commissioners, of course, were very much into the value of the forest for the members of the community. So we did hear that. Getting it refined is where I think I really wish we would have taken the time.

Yeah, so I guess one example that I've seen that piqued my curiosity is the use of social media to try and gather some of this. There's actually a recreation area between the lakes that was using information from Yelp in combination with some of their visitor use monitoring and car counts and things of that nature to try and fill in some of the gaps in their understanding of how the users of their recreation resources feel about them. I think that's a relatively untapped and developing area. I think the private sector is much better able to access it than we are for a variety of reasons, probably most importantly because they can pay for it. But I think there's a lot there that could really help with this, if we had the capacity to work with it. 
That's a big challenge because you're just always kind of scrambling to get stuff done. A local capacity issue. I feel like it probably is getting worse. It's going to vary between one person to the next, but with budget uncertainties, a lot of times you don't hire positions behind people who leave. You've just been informed by somebody else, and depending on that and how Congress chooses to fund the Forest Service, there's a real hesitancy, let's say, to hire behind people a lot of times. There's the fear of are we going to have the budget to continue to pay that person year after year? Right. And, you know, you have the big idea. There's one roadblock or bump, and then it just gets thrown out because we don't have the capacity to make it happen long-term, so we're going to have to go for some lower-hanging fruit.

CHALLENGE: Concept to Reality On-the-Ground Moving from concept to words on a page, since we were all going through this [plan revision] for the first time in two decades, I think we were learning that there's much more to this...This is all supposed to be very high level analysis. It's a comparison between alternatives, but that doesn't mean it's reality. It's very broad brush. It's supposed to be short, simple. Then we write it and it's 400 pages! Kind of like life, I guess. It's easy to make grand sweeping statements and then you have to work through the details.

\section{ANALYTIC META-MEMO: REGIONAL OFFICES}

1. What seems most important or significant to these participants? What stood out most in the interviews?

Recognize the value of social science and values-based public data in forest planning. Feel a bit overwhelmed by the demands put on them as social scientists (time/capacity). See qualitative data as able to engage the public and "tell the story" behind the numbers.

\section{How did the participants talk about the use of social science and/or socio-spatial data or data gaps? Was it said in a positive, negative or neutral context?}

Socio-spatial data engaging the public; finding common ground (shared place).

So we got our public comments and input. We really began to see the value of having these, we'll just call them primitive in the eyes of the public, places. We did ROS mapping. When we mapped for existing, there was very little to nothing that mapped as primitive and very little area that mapped as semi-primitive, but we wanted to preserve that. So what that translated to is then developing a desired ROS. Okay, here's how it's mapped, but here's what the public's telling us. So we are going to actually manage for these settings that may not, according to our protocol, map out, but this is what we want to provide. 
Telling the story (qualitative + quantitative).

But I actually think that there's really huge value in storytelling in general. Not just like the socioeconomic sections, but throughout our whole document, all of our different resource sections...I think what we put out there is a lot of complex information at times. So you kind of miss the story that we're trying to tell. And so I think that qualitative data really helps tell that story and actually could add to the quantitative story. Then people could see that, then maybe they would also value the qualitative information more. Because really, I think, a lot of what we're trying to show the public is what we're struggling with, in terms of balancing our management of the forest. It kind of gets lost sometimes in the numbers.

It's a social construct!

I think that the Forest Service is a social construct. I think that the way we manage lands is also a social construct. I think that the ESA exists because people care about it. It's a social construct. I think the faster the organization can get its head around that notion, the more successful it will be. And the more resilience the agency will have. I think we need to come at it not just from the side of understanding natural resources, but understanding the social construct around that. I think it should drive the whole show.

\section{Was there anything in the interviews that surprised you? What insights did you gain?}

Public Input is NOT Data Collection

Now the collaborative talking points...that's why we've since gone with that, because we know that has been approved...So we collected the data, in a way that it wasn't tallying anything, it was simply designed to be as open ended as possible, again with these facilitated discussion statements, and where the public could come in and offer whatever input they wanted. And we didn't use it in a statistic-type of way. It was nothing more than another way to submit their input.

The "Look at Me!" Culture

But my other theory, this is my theory, is that if you are out there doing good work, good process, it is for the most part quiet, maybe, and it's not a lot of fanfare and you are just getting work done...It doesn't rise to the, "Look at me!" occasion. Maybe we don't give enough credit or something or take enough notice of people's timelines or something. It's weird... We seem to have to wait until somebody takes us to court before it's going to be seen as a good thing or a bad thing....

\section{Did the participants talk about significant links, challenges or opportunities? What were they?}

CHALLENGE: Silos of Expertise 
But I will say there's always that, I kind of laugh about it because it shouldn't be such an effort, it always takes an effort to integrate and work together, even though that's one of the goals of the new planning rule is more integration. But even still, and I think it's just kind of how we're organized and what we're used to doing, each going back to our own desks and looking at it within our lens. So it's only been positive interaction, but it certainly has been a very concerted effort to sit down.

CHALLENGE: OMB Clearance

Part of it is figuring stuff out for the first time. What I mean by that is things like a PPGIS protocol. Clearly it brings a lot of value to the forest plan revision process. But, corporately there's not a lot of organization about how to do that, how to clear OMB requirements. You know we need this OMB clearance. Oh my god, if I could ask for one thing it would be that!

CHALLENGE: Capacity + Time

Part of it is when we all [social scientists] get together at a little meeting and talk about it [improving the reach of social science], it seems like a good idea but as soon as you disperse back into your work that you need to do, you're instantly swallowed up in everything that needs to be done immediately and it's hard to carve time back out to like do the extras. There's so few of us, it's hard to get the capacity to do the extras to get momentum moving on other stuff. You're just so focused on trying to hold it together. I think we're just at this weird critical mass where we, everybody, feels like they're barely holding it together yet we need to do that little bit more so that people do remember that we are important and maybe there should be a few more of us.

LINK: Leadership \& Decision-Making

In a complex organization like the USFS with a strict hierarchy, competent and consistent leadership at each level is critical to effective decision-making and communication between units and levels and smooth functioning within the various departmental and programmatic areas. Hierarchical complexity -- and dysfunction -- can lead to confusion about who decides what, protection of turf, miscommunication or lack of communication, difficulties in sharing information or in interdisciplinary work, etc.

\section{ANALYTIC META-MEMO: RESEARCH STATIONS}

\section{What seems most important or significant to these participants? What stood out most in the interviews?}

Research role of R\&D in the National Forest System -- do research, not write reports. OMB \& PRA challenges in conducting social science research.

Socio-spatial data and tools need to fit a purpose or issue, not simply data collection. 


\section{How did the participants talk about the use of social science and/or socio-spatial data or data gaps? Was it said in a positive, negative or neutral context?}

Issue-Driven vs. Data-Driven Planning

Forest plan development should be a 50,000 foot process; planners who focus on only the data will get into the weeds. Issues, needs and niches at a landscape scale should be determined first, then these needs and issues determine what kind of data (biophysical or social) is needed. This speaks to the FUNCTION of socio-spatial data and data collection tools in the planning process.

They [managers] want a "tool." And when they get that tool, they apply it everywhere...but it's got to be tailored to the particular issue and topic...managers can't do that. It's a conceptual task that is exceedingly difficult for somebody not trained specifically in mapping or the social sciences and how you collect this kind of data...you need to figure out how to create an open-ended tool or an adaptable tool...that leaves the specific questions and methods open to develop based on what the specific problem or issue is...they also have to realize that there has to be a mental-cognitive interaction where they're looking at the tools and asking, 'how do I apply them to my purpose.' So I guess at the grand scale you can identify specific processes and techniques and they've got to be coarse, and they have to understand how it fits into the planning process. But as you scale down, the uses get more scientific, more intricate, more involved, but they also have to be more flexible and more adaptable to a particular issue.

\section{Was there anything in the interviews that surprised you? What insights did you gain?}

The role of research vs. the role of planning:

Scientists write papers...planners write reports.

\section{Did the participants talk about significant links, challenges or opportunities? What were they?}

CHALLENGE: OMB approvals for social science research (e.g. surveys, etc.) Question: Have you identified other barriers that "gee, I would like to get rid of them so I can do my work easier?" Answer: Oh! Honey! Can we talk! The application, the interpretation, of the Paperwork Reduction Act as applying to ethnographic research conducted in support of good forest policy and management is killing me.

It was a conversation we had about 10 years ago now with someone in legislative affairs for the Forest Service. And they said, "Well, if we removed the limits on voluntary things, how many surveys would you all be foisting on the American public in any given year?" And I said, "None." He stops me. And I said, we don't foist. That's the whole point...People want to have a say in how their public lands 
are managed. This [Paperwork Reduction Act - PRA] gets in the way of it. I get that we want to do good quality science. And I get that we don't want to all be doing the same damn thing all over the place and asking the same people the same thing all the time. There's too few of us for that to ever happen... That's the barrier.

CHALLENGE: Resisting pressure from forests to "write reports" rather than "do research."

We are hearing constantly from the national forest system, well, what have you [scientists] done for us lately? It's hard to respond to because it is usually a kneejerk reaction among researchers... We don't work for you. We don't. Our charge, our mission, is not to do research for the national forests. We're supposed to do cutting edge research about forests...And we're also supposed to be looking out for those questions that are going to be biting the national forest managers 10 years from now, not just what's biting them now. So there's a tussle in the agency right now.

I think one of the challenges you face when you're a scientist for the research stations is that you're expected to contribute to the scholarly literature. You're expected to publish peer-reviewed journal articles, for the science community. And those often aren't good products for the managers making decisions. You're also expected to deliver information in a way that's usable by the managers on the ground. Often that could take the form of a general technical report or some sort of a white paper, briefing paper, what not. I always, often, feel like I have to double publish my research results to meet these different sort of audience needs. It's really demanding because I feel like I end up doing one or the other. I either just do the journal article, but I don't do much in the way of transmitting the lessons or results to the managers. Or I just do the applied piece and I never have time to publish the journal article related to the research. It's pretty frustrating in that regard.

LINK: R\&D + Incentives

The other thing is that there are differences between what is science and what is not science in terms of writing a report about something. The National Forest System needs those reports. They need to see the documents. But, for a scientist to work on some of these things would be a career killer... We're paying them to become better at what they do. But if they're doing something that they couldn't write a scientific paper about, to us that is non-effective use of their time... And that's sometimes hard for people who want them to do something different to understand. 


\section{Appendix K: Thematic Network Organizing Criteria}

\section{Barriers \& Opportunities in the Collection \& Use of Socio-Spatial Data in Forest Planning THEMATIC NETWORK ORGANIZING CRITERIA}

FINAL CODES: Policy \& Law; Org Culture; Org Capacity; Org Leadership; Org Strategy (Planning); Org Budget; Research/BASI; Science Networking; Public Engagement; Socio-Spatial Data

Sorting Criteria \#1: Systems Lens -- what parts of the system does the theme address? Do they address functional elements, flows of information or resources, inputs into the systems, principles that guide operations, outputs or purpose?

Sorting Criteria \#2: Study Objective -- how close or potentially impactful is the theme to the study objective? What is the theme's explanatory power?

\begin{tabular}{|c|c|c|c|c|}
\hline $\begin{array}{l}\text { FINAL } \\
\text { CODES }\end{array}$ & $\begin{array}{l}\text { BASIC THEMES (ranked by } \\
\text { "closeness" to objective) }\end{array}$ & $\begin{array}{c}\text { BASIC } \\
\text { THEME } \\
\text { LABEL }\end{array}$ & $\begin{array}{l}\text { ORGANIZING } \\
\text { THEMES }\end{array}$ & GLOBAL THEME \\
\hline $\begin{array}{l}\text { Org } \\
\text { Leadership } \\
\text { Org } \\
\text { Capacity } \\
\text { Org Budget } \\
\text { Org Strategy } \\
\text { \& Planning } \\
\text { Socio- } \\
\text { Spatial Data }\end{array}$ & $\begin{array}{l}\text { 9. Socio-spatial data belongs in the } \\
\text { public sensing phase of planning to } \\
\text { better engage the public as it would } \\
\text { contribute valuable values-based } \\
\text { data in the development of the Need } \\
\text { for Change and Desired Conditions } \\
\text { statements; challenges are directly } \\
\text { (capacity). } \\
\text { 10. The paucity of staff in the } \\
\text { regional office and forests with } \\
\text { social science backgrounds and } \\
\text { expertise limits the ability to collect } \\
\text { socio-spatial data (capacity). } \\
\text { 5. Good leadership enables effective } \\
\text { and efficient planning. } \\
6 \text {. Budget reductions are directly } \\
\text { linked to staff capacity; affects } \\
\text { prioritization in planning strategies } \\
\text { that prevent collecting more socio- } \\
\text { spatial data for plan revision (low } \\
\text { hanging fruit). }\end{array}$ & $\begin{array}{l}\begin{array}{l}\text { Planning } \\
\text { Decisions }\end{array} \\
\text { Few Social } \\
\text { Scientists } \\
\text { Leadership } \\
\text { Budget } \\
\text { Reductions } \\
\text { Planning } \\
\text { Decisions }\end{array}$ & $\begin{array}{l}\text { Staff Capacity } \\
\text { Formal System } \\
\text { Elements -- } \\
\text { Budget \& Staff }\end{array}$ & \multirow[t]{2}{*}{$\begin{array}{l}\text { All themes suggest a } \\
\text { desire to improve the } \\
\text { collection \& use of } \\
\text { socio-spatial data. } \\
\text { Socio-Spatial Data } \\
\text { Improves Forest } \\
\text { Planning } \\
\text { *Tools } \\
\text { *Data Types } \\
\text { *Decision Impacts }\end{array}$} \\
\hline $\begin{array}{l}\text { Org Culture } \\
\text { Org Strategy } \\
\text { \& Planning } \\
\text { Public } \\
\text { Engagement } \\
\text { Research/ } \\
\text { BASI } \\
\text { Science } \\
\text { Networking } \\
\text { Socio- } \\
\text { Spatial Data }\end{array}$ & $\begin{array}{l}\text { 10. The paucity of staff in the } \\
\text { regional office and forests with } \\
\text { social science backgrounds and } \\
\text { expertise limits the ability to collect } \\
\text { socio-spatial data (capacity). } \\
\text { 9. Socio-spatial data belongs in the } \\
\text { public sensing phase of planning to } \\
\text { better engage the public and inform } \\
\text { the need for change and desired } \\
\text { conditions statements; inability to } \\
\text { gather this data (capacity). } \\
\text { 8. Data access and a social science } \\
\text { support portal are needed to more } \\
\text { effectively transmit knowledge } \\
\text { about the collection and use of }\end{array}$ & $\begin{array}{l}\text { Data Collection } \\
\text { Staff Capacity } \\
\text { Data Collection } \\
\text { Planning } \\
\text { Decisions } \\
\text { Staff Capacity } \\
\text { Data Portal } \\
\text { Staff Capacity } \\
\text { Data Gaps } \\
\text { (local) } \\
\text { Data Gaps } \\
\text { (external) }\end{array}$ & $\begin{array}{l}\text { Data Collection, } \\
\text { Access \& } \\
\text { Dissemination } \\
\text { System Flows -- } \\
\text { Information }\end{array}$ & \\
\hline
\end{tabular}




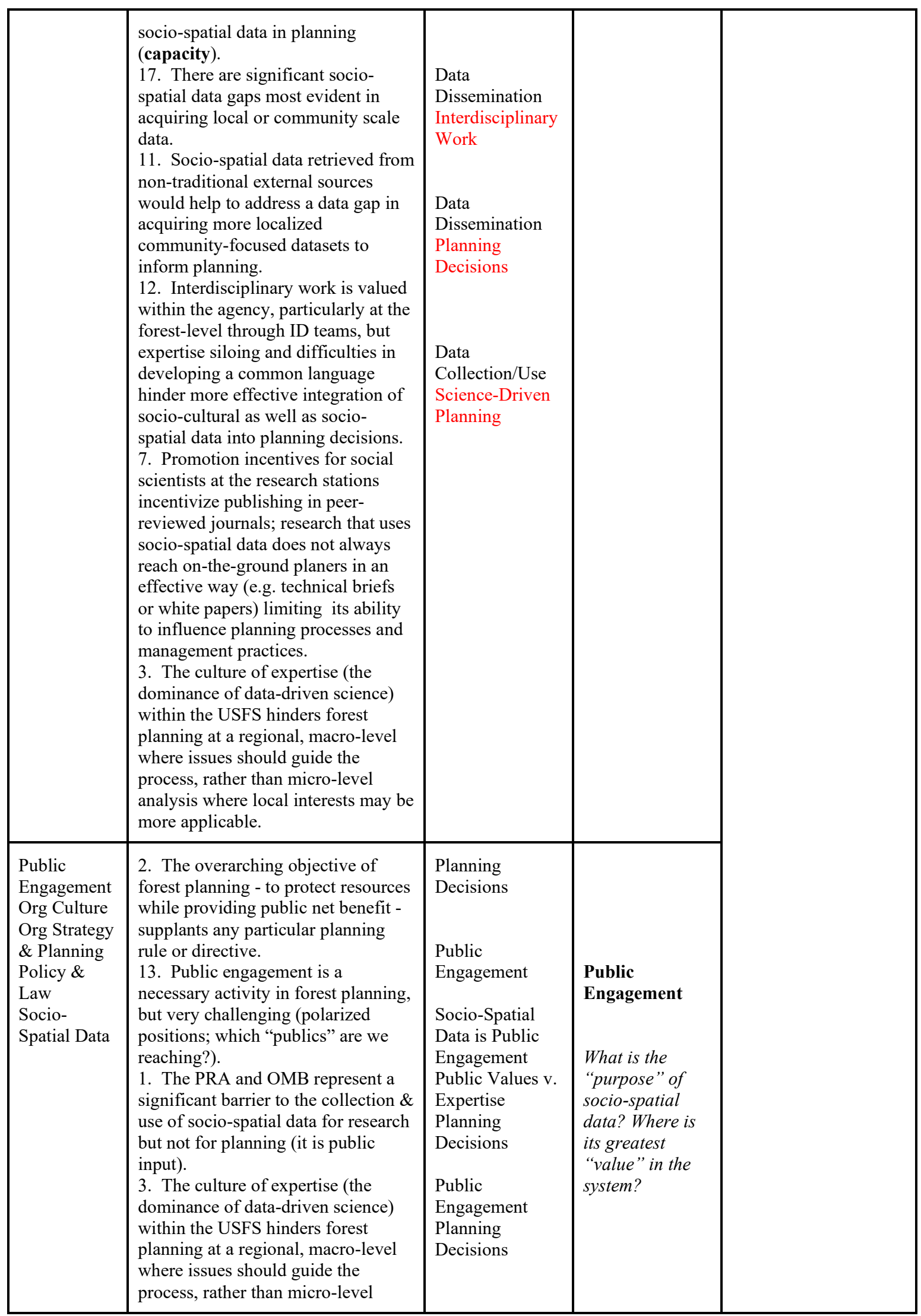




\begin{tabular}{|c|c|c|c|c|}
\hline & $\begin{array}{l}\text { analysis where local interests are } \\
\text { more applicable. } \\
\text { 4. The importance of science-based } \\
\text { decision-making is not questioned; } \\
\text { the culture of expertise if linked to } \\
\text { challenges affecting organizational } \\
\text { efficiency, authentic public } \\
\text { engagement, and the tendency to de- } \\
\text { prioritize (not necessarily de-value) } \\
\text { socio-spatial data in forest planning. }\end{array}$ & & & \\
\hline $\begin{array}{l}\text { Socio- } \\
\text { Spatial Data }\end{array}$ & $\begin{array}{l}\text { 14. Socio-spatial data collection } \\
\text { tools take many forms; many data } \\
\text { formats.. } \\
\text { 15. Socio-spatial data collections } \\
\text { tools are employed at different } \\
\text { points in the planning process and } \\
\text { for different purposes. } \\
\text { 16. Socio-spatial data influences } \\
\text { decision-making in both direct and } \\
\text { indirect ways. }\end{array}$ & $\begin{array}{l}\text { Data } \\
\text { Formats } \\
\text { Data Tools } \\
\text { Data } \\
\text { Impacts }\end{array}$ & System Outputs & \\
\hline
\end{tabular}

\section{Socio-Spatial Data Thematic Network: Organizing Criteria}

Using the language of thematic networking, keywords assigned to the meta-themes became the "basic themes." I applied two criteria to organize these themes. The first applied a systems lens to each theme to determine what components of the system the theme addressed (e.g. a functional element, a process mechanism, a flow of resources, an operating principle, etc). For example, the public input theme refers to the process of public engagement. Staff incentives is a structural element of the system related to formalized operating procedures. Data portal refers to a mechanism in which information is exchanged. This helped to initially group the themes by their operative purpose within the organization.

For the second criterion, I used the study objective - understanding the collection and use of socio-spatial data in forest planning - and assessed how "close" the theme was in relation to the objective. For example, the themes labeled as data portal and data gaps refer directly to sociospatial data whereas leadership is more tangentially related. The budget theme is several steps removed from a direct impact; the budget affects capacity (in time and expertise) which then affects the ability of staff to collect, interpret and use socio-spatial data. Applying this criterion resulted in sorting the themes into related groupings with a loose hierarchy indicating which themes have potentially more explanatory power than others.

Three "organizing themes" emerged from applying these two sorting criteria: (1) Data Collection, Use \& Dissemination; (2) Public Engagement; and (3) Staff Capacity. Each of the basic themes could be assigned to one of these organizing themes; two themes were applicable to more than one. The paucity of social science staff theme obviously links directly to the Staff Capacity organizing theme, but also the Data Collection, Use and Dissemination theme as it affects the ability of staff to "stay on top of" what data is available and how to use it. In addition, the planning decisions theme relates both to Staff Capacity (lack of capacity often results in prioritizing only the "low hanging fruit") and also to the Data Collection theme (deliberate planning decisions are made about what data to collect, when and for what purpose). 
The final step in creating a thematic network is to reflect upon the organizing themes and their associated basic themes and ask, what unifies them all? What is an underlying statement that each theme speaks to? After much reflection and rereading each theme, I kept coming back to the impetus for this study in the first place - affirmation from USFS planners and scientists that they see value in socio-spatial data, but limited understanding around how this data is actually being collected and used or what specific barriers were being encountered that may hinder data collection efforts. As a global theme, socio-spatial data improves forest planning, represents a fundamental premise that is both explicit and implicit in the themes that make up this dataset. All participants spoke of integrating public values, attitudes and land-use preferences as a necessary component in forest planning and that a better understanding of the depth and breadth of these values and uses would improve management decisions and practices. This sentiment was true among the natural and social scientists that participated in the study as well as between scientists and practitioners at all levels. 